\title{
IFN $\beta$ as regulator of \\ CD14/TLR4-mediated microglial responses to CNS infection and damage
}

\author{
Doctoral Thesis \\ In partial fulfillment of the requirements for the degree \\ "Doctor rerum naturalium (Dr. rer. nat.)" \\ in the Molecular Medicine Study Program \\ at the Georg-August-University Göttingen
}

submitted by

Christin Döring

born in Rotenburg/Fulda

Göttingen, December 2016 



\section{Members of the Thesis Committee}

\section{Supervisor}

Prof. Dr. Wolfgang Brück

Department of Neuropathology

University Medical Center, Georg-August-University Göttingen

\section{Second member of the Thesis Committee}

Prof. Dr. Jürgen Wienands

Institute of Cellular and Molecular Immunology

University Medical Center, Georg-August-University Göttingen

\section{Third member of the Thesis Committee}

Prof. Dr. Dr. Hannelore Ehrenreich

Department of Clinical Neurosciences

Max Planck Institute for Experimental Medicine, Göttingen

Date of Disputation: 



\section{Affidavit}

Here I declare that my doctoral thesis entitled "IFN $\beta$ as regulator of CD14/TLR4-mediated microglial responses to CNS infection and damage" has been written independently with no other sources and aids than quoted.

Christin Döring

Göttingen, December 2016 



\section{List of Publications}

Janova H, Böttcher C, Holtman IR, Regen T, van Rossum D, Götz A, Ernst AS, Fritsche C, Gertig U, Saiepour N, Gronke K, Wrzos C, Ribes S, Rolfes S, Weinstein J, Ehrenreich H, Pukrop T, Kopatz J, Stadelmann C, Salinas-Riester G, Weber MS, Prinz M, Brück W, Eggen BJ, Boddeke HW, Priller J, Hanisch UK. CD14 is a key organizer of microglial responses to CNS infection and injury. Glia. 2016 Apr; 64(4):635-49. doi: 10.1002/glia.22955

Döring C, Regen T, Gertig U, van Rossum D, Saiepour N, Brück W, Janova H, Hanisch UK. A presumed antagonistic LPS identifies distinct functional organization of TLR4 in mouse microglia. Submitted. 



\section{Table of Contents}

Abstract I

Acknowledgment II

List of Figures III

List of Tables $\quad$ V

$\begin{array}{lll}\text { Abbreviations } & \text { VI }\end{array}$

1 Introduction 1

1.1 Inflammation and innate immunity $\ldots \ldots \ldots \ldots \ldots \ldots$

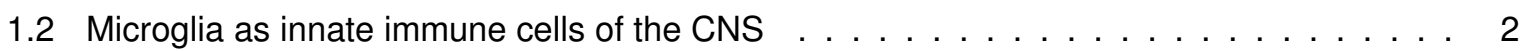

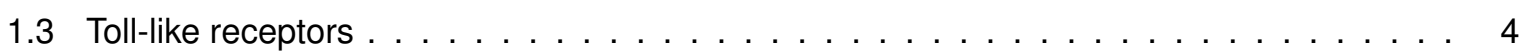

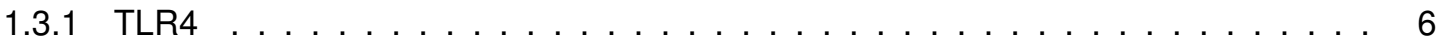

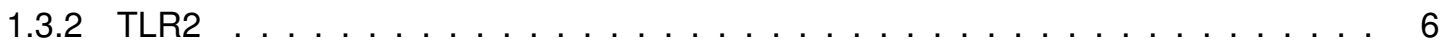

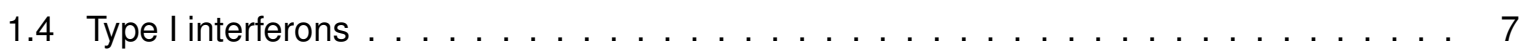

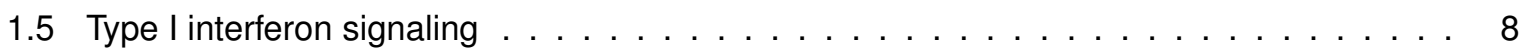

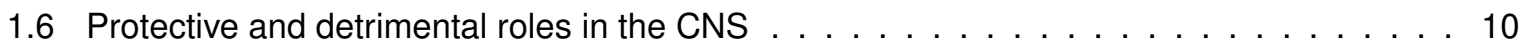

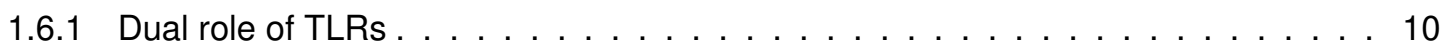

1.6.2 Dual role of interferons . . . . . . . . . . . . . . . . . . . . . 11

2 Aim of the study 13

3 Material and Methods $\quad 14$

3.1 Material . . . . . . . . . . . . . . . . . . . . . . . . . . . 14

3.1 .1 Reagents . . . . . . . . . . . . . . . . . . . . . 14

3.1.2 Buffers, solutions and cell culture media . . . . . . . . . . . . 16

3.1 .3 Antibodies . . . . . . . . . . . . . . . . . . . . . . . . . . . . 17

3.1 .4 Applied kits . . . . . . . . . . . . . . . . . . . . . . . 18

3.1 .5 Primer for real-time $\mathrm{PCR} \ldots \ldots \ldots \ldots \ldots \ldots$

3.1 .6 Consumables . . . . . . . . . . . . . . . . . . . . . . . . . . . 19

3.1.7 Software and technical devices . . . . . . . . . . . . . . . . . . 19

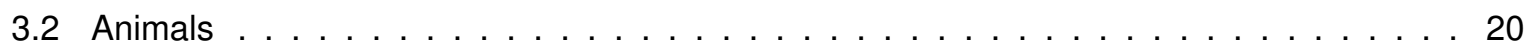

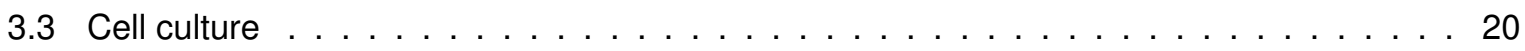

3.3.1 Primary mouse microglial cultures . . . . . . . . . . . . . . 20

3.3.2 Bone marrow-derived macrophage cultures . . . . . . . . . . . . . . . . . . 21

3.3.3 Peritoneal macrophage cultures . . . . . . . . . . . . . . . . . . 21

3.3.4 L929 fibroblast cultures . . . . . . . . . . . . . . . . . . . . . . . . . . 21 
3.4 In vitro stimulation of cells and determination of cell viability . . . . . . . . . . . 22

3.5 Quantification of chemokines and interferons by ELISA . . . . . . . . . . . . . . . 22

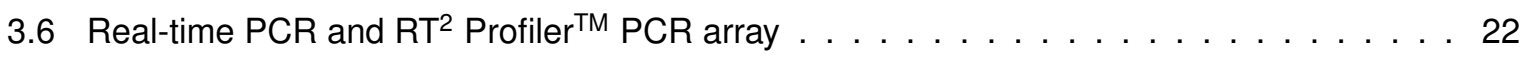

3.7 Flow cytometry analysis of E.coli and myelin phagocytosis . . . . . . . . . . . . 23

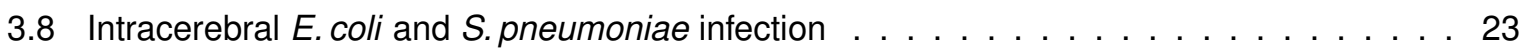

3.9 Induction of focal NMO-like lesions in mice . . . . . . . . . . . . . . . . 24

3.10 Preparation of single-cell suspensions from brain tissue for flow cytometry analysis . . . 24

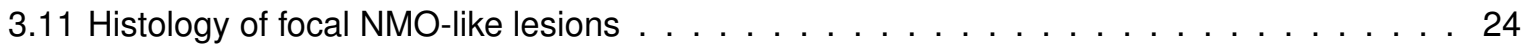

3.11 .1 Preparation for histology . . . . . . . . . . . . . . . . . . . 24

3.11 .2 Hematoxylin and eosin $(\mathrm{HE})$ staining . . . . . . . . . . . . . . . . . . . . . . . . . . . 25

3.11 .3 Aquaporin 4 (AQP4) staining . . . . . . . . . . . . . . . . . . . 25

3.11.4 Chloroacetate esterase (CAE) enzyme histochemistry and acquisition of neutrophil numbers . . . . . . . . . . . . . . . . . . . . . . . 25

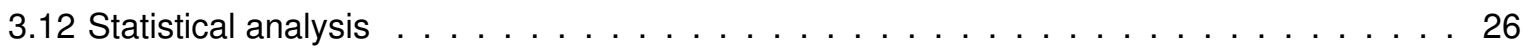

4 Results $\quad 27$

4.1 Microglial production of type I interferons . . . . . . . . . . . . . . . . 27

4.1.1 Microglia produce IFN $\beta$, but hardly any IFN $\alpha$ upon TLR stimulation . . . . . . . 27

4.1 .2 IFN $\beta$ production is TRIF-dependent . . . . . . . . . . . . . . 29

4.1.3 IFN $\beta$ production is absent in CD14-deficient microglia . . . . . . . . . . 30

4.2 Type I interferon signaling controls TLR4-induced chemokine production . . . . . . . . 31

4.2.1 Regulation of CXCL1 and CCL2 production depends on IFNAR1 . . . . . . . 31

4.2.2 IFNAR1 deficiency causes excessive neutrophil infiltration into the brain of E. coliinfected mice . . . . . . . . . . . . . . . . . . 32

4.2.3 IFNAR1 deficiency does not impair microglial phagocytosis . . . . . . . . . . 33

4.2.4 Neutrophil infiltration in a model of focal NMO is independent of IFNAR1 . . . . . 34

4.2.5 IFNAR1-deficient mice display lower T cell numbers in the brain . . . . . . . . 37

4.2.6 Altered chemokine production in IFNAR1-deficient microglia is not rescued by time 38

4.2.7 IFNAR1 deficiency leads to similar changes in the chemokine production of microglia and macrophages . . . . . . . . . . . . . . . . . . . . 39

4.2.8 IFNAR1 deficiency can be phenocopied by functional block . . . . . . . . . . . 40

4.2.9 Regulation of CXCL1 and CCL2 production depends on IFNAR2 . . . . . . . . 40

4.2.10 Functional absence of IFNAR1 and IFNAR2 exceeds effects of either deficiency . 41

4.2.11 Regulation of CXCL1 and CCL2 production depends on janus kinases . . . . . . 42

4.2.12 Tyk2 ${ }^{E 775 K}$ microglia show properties that deviate from the previous results . . . . . 44

4.2.13 Regulation of CXCL1 and CCL2 production depends on STAT1 and STAT2 . . . 46

4.3 IFNAR controls TLR2-induced chemokine production . . . . . . . . . . . . . 50

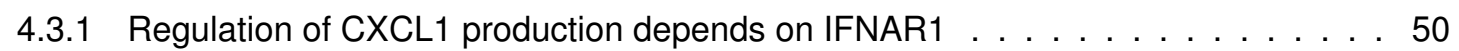

4.3.2 IFNAR1 deficiency does not affect neutrophil infiltration into the brain of S. pneumoniae-infected mice . . . . . . . . . . . . . . . . . . 5 50

4.3.3 Altered chemokine production in IFNAR1-deficient microglia is not rescued by time 52

4.3.4 IFNAR1 deficiency leads to similar changes in the chemokine production of microglia and bone marrow-derived, but not peritoneal macrophages . . . . . . . 5 53

4.3.5 IFNAR1 deficiency can be partially phenocopied by functional block . . . . . . 53

4.3.6 Regulation of CXCL1 production depends on IFNAR2 . . . . . . . . . . . . . 54

4.3.7 Functional absence of IFNAR1 and IFNAR2 exceeds effects of either deficiency . 54

4.3.8 Regulation of CXCL1 production is independent of janus kinases . . . . . . . . 55 
4.3.9 Tyk2 ${ }^{E 775 K}$ microglia show properties that deviate from the previous results . . . . . 57 4.3.10 Regulation of CXCL1 production is independent of STAT1 and STAT2 . . . . . . . 58

4.4 Type I interferon signaling controls TLR3-induced chemokine production . . . . . . . . 60

5 Discussion $\quad 63$

5.1 Microglia mainly produce IFN $\beta$ in response to activation of different TLRs . . . . . 63

5.2 IFNAR controls chemokine production in response to TLR4, TLR2 and TLR3 . . . . . 65

5.3 IFNAR1 regulates the infiltration of neutrophils in a model of gram-negative, but not gram-

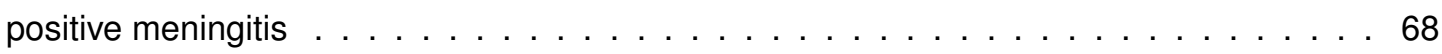

5.4 The infiltration of neutrophils into focal NMO-like lesions is independent of IFNAR1 . . . 70

5.5 Janus kinases control chemokine production in response to TLR4, but not TLR2 . . . . 72

5.6 STAT1 and STAT2 control chemokine production in response to TLR4 and TLR3, but not

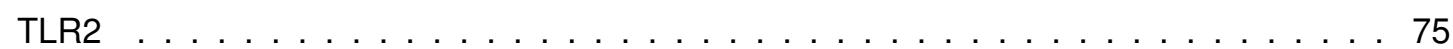

5.7 Type I interferon signaling controls TLR4-induced chemokine production, while responses to TLR2 are regulated by IFNAR $\ldots \ldots \ldots \ldots$. . . . . . . . . . . . . . .

6 Summary and Conclusions $\quad 80$

Bibliography $\quad$ IX 



\section{Abstract}

Microglia are the innate immune cells of the central nervous system (CNS). They are capable of sensing infection and damage through various receptors and consequently trigger an appropriate immune response. This includes the production of several cytokines and chemokines that modulate immune functions and can lead to the recruitment of peripheral immune cells into the CNS. Among these, the neutrophil chemoattractant $C X C L 1$ and the monocyte chemoattractant CCL2 are released in response to activation of Toll-like receptors (TLRs) as sensors of highly conserved structures on foreign as well as host molecules. Apart from that, microglia have been demonstrated to secrete interferons (IFNs), which are crucial cytokines for fighting viral and also bacterial infections.

Here we show that the production of type I interferons can be induced by activation of microglial TLRs. In response to double-stranded RNA associated with viral infections detected by TLR3, microglia are able to produce IFN $\alpha$ as well as IFN $\beta$. Furthermore, activation of TLR4 by bacterial ligands and damagerelated factors triggers IFN $\beta$ release. In contrast to that, bacterial ligands of TLR2 fail to induce any type I interferon. These differences in interferon production can be attributed to the differential involvement of the major TLR signaling routes. We demonstrate that the release of IFN $\beta$ exclusively depends on the presence of the TIR-domain-containing adapter-inducing IFN $\beta$ (TRIF), which is only recruited upon activation of TLR3 and TLR4. Within the TLR4 system, TRIF-dependent signaling further requires the TLR4 co-receptor CD14 and accordingly, we could show that CD14 enables IFN $\beta$ production.

Following their release, type I interferons exert a variety of functions. In addition to their role in host defenses against infections, several immunomodulatory effects have been identified, which can be either beneficial or detrimental depending on the disease context. Here we show, how IFN $\beta$ differentially regulates microglial responses to CNS infection and damage. We demonstrate that the production of CXCL1 and CCL2 in response to TLR activation is controlled by an IFN $\beta$-mediated feedback mechanism. While CCL2 is positively regulated, CXCL1 is under negative control of type I interferon signaling. This mechanism is individually organized within different TLR systems. In response to TLR4 activation, both subunits of the interferon- $\alpha / \beta$ receptor (IFNAR) cooperatively regulate chemokine production. This effect is mediated by the canonical type I interferon signaling pathway, which includes janus kinases and the signal transducer and activator of transcription (STAT)1 and STAT2. In contrast to that, only IFNAR1 and IFNAR2 regulate TLR2-induced chemokine production independent of downstream components of the canonical type I interferon signaling pathway.

These different regulatory mechanisms of chemokine production also translate into in vivo control of immune cell infiltration during CNS infection. In a model of gram-negative meningitis, we demonstrate protective effects of IFNAR1 by prevention of excessive neutrophil infiltration into the brain. This correlates with the IFN $\beta$-mediated negative control of microglial CXCL1 production. In contrast to that, neutrophil infiltration in models of gram-positive meningitis and the autoimmune disease neuromyelitis optica is not controlled by type I interferon signaling. This underlines that immunomodulatory effects of type I interferons are very specific and highlights the importance of understanding their mode of action. 


\section{Acknowledgment}

First of all, I would like to thank a great person even though he tragically cannot read this anymore. Prof. Dr. Uwe Hanisch was a fantastic supervisor, excellent teacher and inspiring scientist. He raised my interest in the fascinating topic of microglia and guided me through the first steps of my scientific career. He taught me to be always curious and question established facts. I really appreciate the time we worked together.

A special thanks goes to Prof. Dr. Wolfgang Brück, who gave me the opportunity to continue my project and finish my thesis under his supervision. I am grateful for the time he spent for scientific discussions and all his further support.

Furthermore, I want to thank the members of my thesis committee Prof. Dr. Jürgen Wienands and Prof. Dr. Dr. Hannelore Ehrenreich for their helpful ideas, comments and questions during my progress reports.

Many thanks to our group!! Thanks to Elke Pralle and Susanne Kiecke for supporting me all the time. You did not only help me with all the laboratory work, but also took care of me and always had a sympathetic ear. Thanks to Ulla Gertig for the good time from the very beginning until we both finished. I am really grateful that you always listened to me, helped me understanding all the mysterious data and I am happy that we became friends. Our trip to Keystone and Denver during the hottest summer ever was awesome! Thanks also to the former members of our group Dr. Hana Janova and Dr. Nasrin Saiepour for all the things I learned from them.

Apart from that, I want to thank all the people, who contributed to my project: Dr. Jana Seele and especially Dr. Sandra Ribes for all the help with the bacterial meningitis models; Dr. Anne Winkler, who made my work with the focal NMO model possible; Dr. Reeza Khorooshi and Prof. Trevor Owens for the IFN $\alpha$ measurements; my students Lisa Linhoff and Sebastian Torke for their assistance; Prof. Marco Prinz and Prof. Thomas Meyer for generously providing knock-out mice and scientific support.

I would like to thank all people in my office: Insa, Sarah, Basti, Linda and Kim. We had a great time with lots of coffee, much to laugh about and nice evenings. Thanks to all other colleagues and friends for the enjoyable working atmosphere and the pleasant time in Göttingen. Many thanks to all the people, who spent their time with proofreading of my thesis: Maike, Tobias, Florian, Sarah, Anne and Jana.

Finally, I want to thank my family - my dad, grandparents and parents-in-law. Most importantly, my wonderful husband Florian deserves my greatest thanks. During all the years, you were always there for me. Thanks for all the motivation and positive thinking. You and me together have always been the best team of the world! $\odot$ 


\section{List of Figures}

1.1 The canonical type $\mathrm{I}$ interferon signaling pathway $\ldots \ldots \ldots \ldots \ldots$

4.1 Microglia produce IFN $\beta$ upon stimulation with several TLR agonists. . . . . . . . . 28

4.2 Microglia produce IFN $\alpha$ only in response to TLR3 stimulation. . . . . . . . . . . . . 29

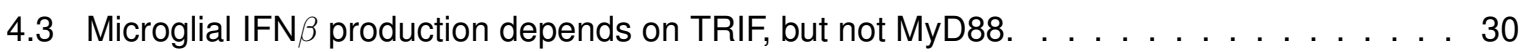

4.4 IFN $\beta$ production is completely absent in $c d 14^{-}$microglia. . . . . . . . . . . . 30

4.5 IFNAR1 deficiency causes increased CXCL1 and decreased CCL2 production by TLR4stimulated microglia, but does not affect IFN $\beta$ levels. . . . . . . . . . . . . . . 32

4.6 Neutrophil infiltration into the brain of E. coli-infected ifnar $1^{\%}$ mice is higher than in wt mice. 33

4.7 The phagocytic capacity does not differ between wt and ifnar ${ }^{\%}$ microglia. . . . . . . 34

4.8 Intracortical injection of NMO-Ab together with human complement leads to the formation of astrocyte-depleted lesions and infiltration of neutrophils. . . . . . . . . . . . 35

4.9 Neutrophil infiltration into focal NMO-like lesions is not affected by IFNAR1 deficiency. . . 36

4.10 Intracerebral CXCL1 production is similarly induced in wt and ifnar1 ${ }^{-/}$mice with focal NMO-like lesions. . . . . . . . . . . . . . . . . . . . . . . . . . 37

4.11 IFNAR1-deficient mice display lower T cell numbers in the brain following different treat-

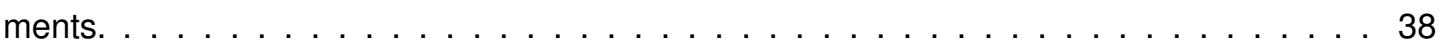

4.12 Altered CXCL1 and CCL2 production by ifnar $1^{-/}$microglia in response to TLR4 stimulation is stable over time. . . . . . . . . . . . . . . . . . . . . . 39

4.13 IFNAR1 deficiency similarly affects TLR4-induced CXCL1 and CCL2 production by microglia, BMDM and $\mathrm{pM} \Phi . \ldots \ldots \ldots \ldots$. . . . . . . . . . . . . . . . . . . . . . . . .

4.14 Functional block of IFNAR1 increases microglial CXCL1 and decreases CCL2 levels, but does not affect IFN $\beta$ production in response to TLR4 stimulation. . . . . . . . . . . . . 40

4.15 Functional block of IFNAR2 increases microglial CXCL1 and decreases CCL2 levels, but does not affect IFN $\beta$ production in response to TLR4 stimulation. . . . . . . . . . . . 41

4.16 Functional absence of IFNAR1 and IFNAR2 cooperatively affects microglial CXCL1, but not CCL2 production in response to TLR4 stimulation. . . . . . . . . . . . . . . . . 42

4.17 Janus kinase inhibition increases CXCL1 and decreases CCL2 production in response to TLR4 stimulation in a dose-dependent manner. . . . . . . . . . . . . . . . 43

4.18 Tyk2 ${ }^{E 775 K}$ microglia produce less CXCL1, more CCL2 and slightly less IFN $\beta$ than wt cells in response to TLR4 stimulation. . . . . . . . . . . . . . . . . . . . . . . . 44

4.19 $\mathrm{Tyk}^{\mathrm{E}}{ }^{\mathrm{E} 75 \mathrm{~K}}$ microglia are responsive to IFN $\beta$-mediated regulation of TLR4-induced CXCL1

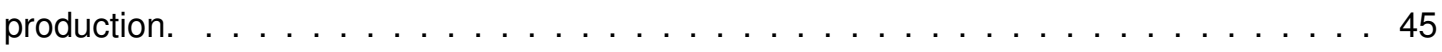

4.20 Janus kinase inhibition decreases TLR4-induced CCL2 production by tyk $2^{E 775 K}$ microglia in a dose-dependent manner, but does not affect CXCL1 release by these cells. . . . . . 46

4.21 Expression of STAT1 and STAT2, but not STAT3 is up-regulated in response to TLR4 stimulation. 
4.22 STAT1 deficiency causes increased CXCL1 and decreased CCL2 as well as IFN $\beta$ production by TLR4-stimulated microglia. . . . . . . . . . . . . . . . . . . . . . 47

4.23 Stat $1 \%$ microglia are less responsive to IFN $\beta$-mediated regulation of TLR4-induced CXCL1 production than wt cells. . . . . . . . . . . . . . . . . . 48

4.24 STAT2 deficiency causes increased CXCL1 and decreased CCL2 production by TLR4stimulated microglia, but does not affect IFN $\beta$ levels. . . . . . . . . . . . . . . . . . 48

4.25 Stat $^{\%}$ microglia are less responsive to IFN $\beta$-mediated regulation of TLR4-induced CXCL1 production than wt cells. . . . . . . . . . . . . . . . . . . . . . . . 49

4.26 Expression of STAT1, STAT2 and STAT3 is not changed by TLR4 stimulation in stat ${ }^{-1}$ and stat $^{-/}$microglia. . . . . . . . . . . . . . . . . . . . . . . 49

4.27 While CCL2 is in general not released in response to TLR2 stimulation, IFNAR1 deficiency causes increased CXCL1 production by TLR2-stimulated microglia. . . . . . . . . 50

4.28 Neutrophil infiltration into the brain of $S$. pneumoniae-infected mice is not affected by IFNAR1 deficiency. . . . . . . . . . . . . . . . . . . . . . . . . . 51

4.29 Altered CXCL1 and CCL2 production by ifnar ${ }^{-1}$ microglia in response to TLR2 stimulation is stable over time. . . . . . . . . . . . . . . . . . . . . . . . . . 52

4.30 IFNAR1 deficiency similarly affects TLR2-induced CXCL1 production by microglia and BMDM, but not by $\mathrm{pM} \Phi$.

4.31 Functional block of IFNAR1 increases microglial CXCL1 production in response to MALP2, but not Pam3CSK4 stimulation. . . . . . . . . . . . . . . . . . . 53

4.32 Functional block of IFNAR2 increases microglial CXCL1 production in response to TLR2 stimulation.

4.33 Functional absence of IFNAR1 and IFNAR2 cooperatively affects microglial CXCL1 production in response to TLR2 stimulation. . . . . . . . . . . . . . . . . . . . 55

4.34 Janus kinase inhibition does not affect CXCL1 and CCL2 production in response to TLR2 stimulation in a dose-dependent manner. . . . . . . . . . . . . . . . . 56

4.35 Tyk2 ${ }^{E 775 K}$ microglia produce less CXCL1 than wt cells in response to TLR2 stimulation and this production can be even lowered by $\operatorname{IFN} \beta$ treatment. . . . . . . . . . . . . . . 57

4.36 Janus kinase inhibition does not affect TLR2-induced CXCL1 production by tyk2 ${ }^{E 775 K}$

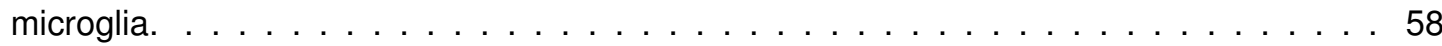

4.37 Expression of STAT1, STAT2 and STAT3 is not changed by TLR2 stimulation in wt, stat1 ${ }^{-1}$ and stat $^{-/}$microglia. . . . . . . . . . . . . . . . . . . . . . 59

4.38 STAT1 and STAT2 deficiency do not affect CXCL1 production by TLR2-stimulated microglia. 59

4.39 While CXCL1 is in general not released in response to TLR3 stimulation, IFNAR1 deficiency causes decreased CCL2 production by TLR3-stimulated microglia. . . . . . . . . 60

4.40 IFNAR1 deficiency similarly affects TLR3-induced CCL2 production by microglia and BMDM, but not by $\mathrm{pM} \Phi . \ldots \ldots \ldots \ldots$. . . . . . . . . . . . . . . . . 61

4.41 STAT1 and STAT2 deficiency cause decreased CCL2 production by TLR3-stimulated mi-

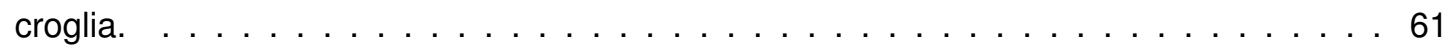

4.42 Deficient type I interferon signaling impairs IFN $\beta$ production by TLR3-stimulated microglia. 61

5.1 Proposed mechanism for the regulation of TLR4-induced chemokine production by the canonical type I interferon signaling pathway . . . . . . . . . . . . . . . . . 79 


\section{List of Tables}

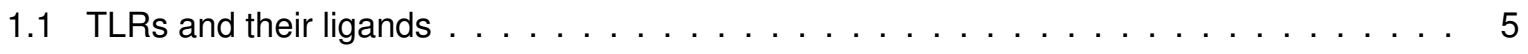

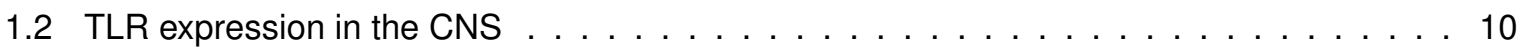

3.1 Reagents for in vitro stimulation of cells $\ldots \ldots \ldots \ldots \ldots$

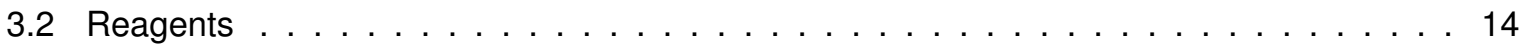

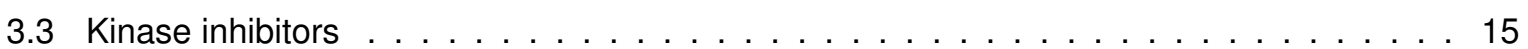

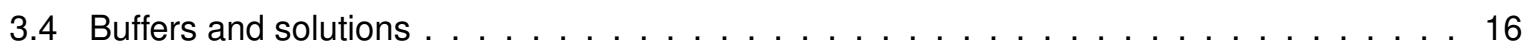

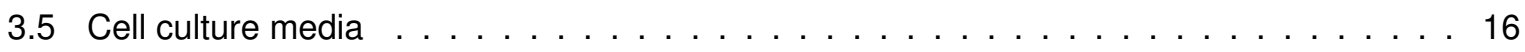

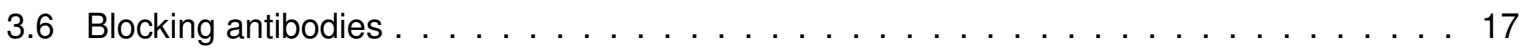

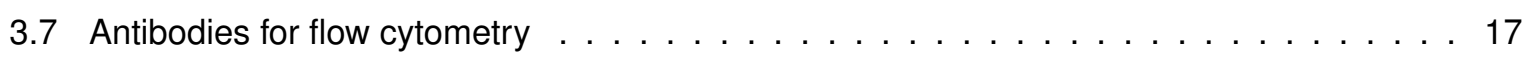

3.8 Antibodies for induction of focal NMO-like lesions $\ldots \ldots \ldots \ldots \ldots$

3.9 Immunohistochemistry antibodies . . . . . . . . . . . . . . . . . . 17

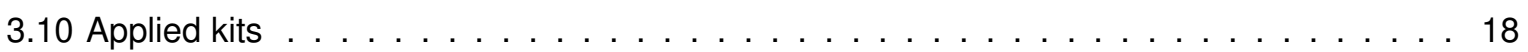

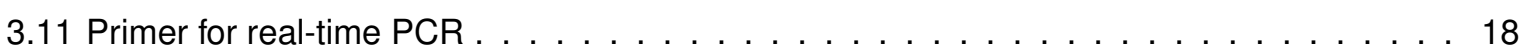

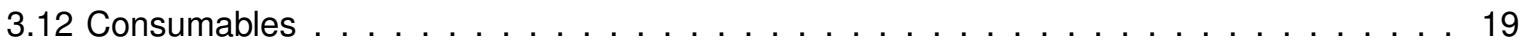

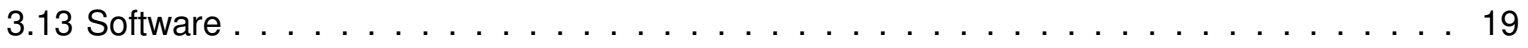

3.14 Technical devices . . . . . . . . . . . . . . . . . . . . . 20 


\section{Abbreviations}

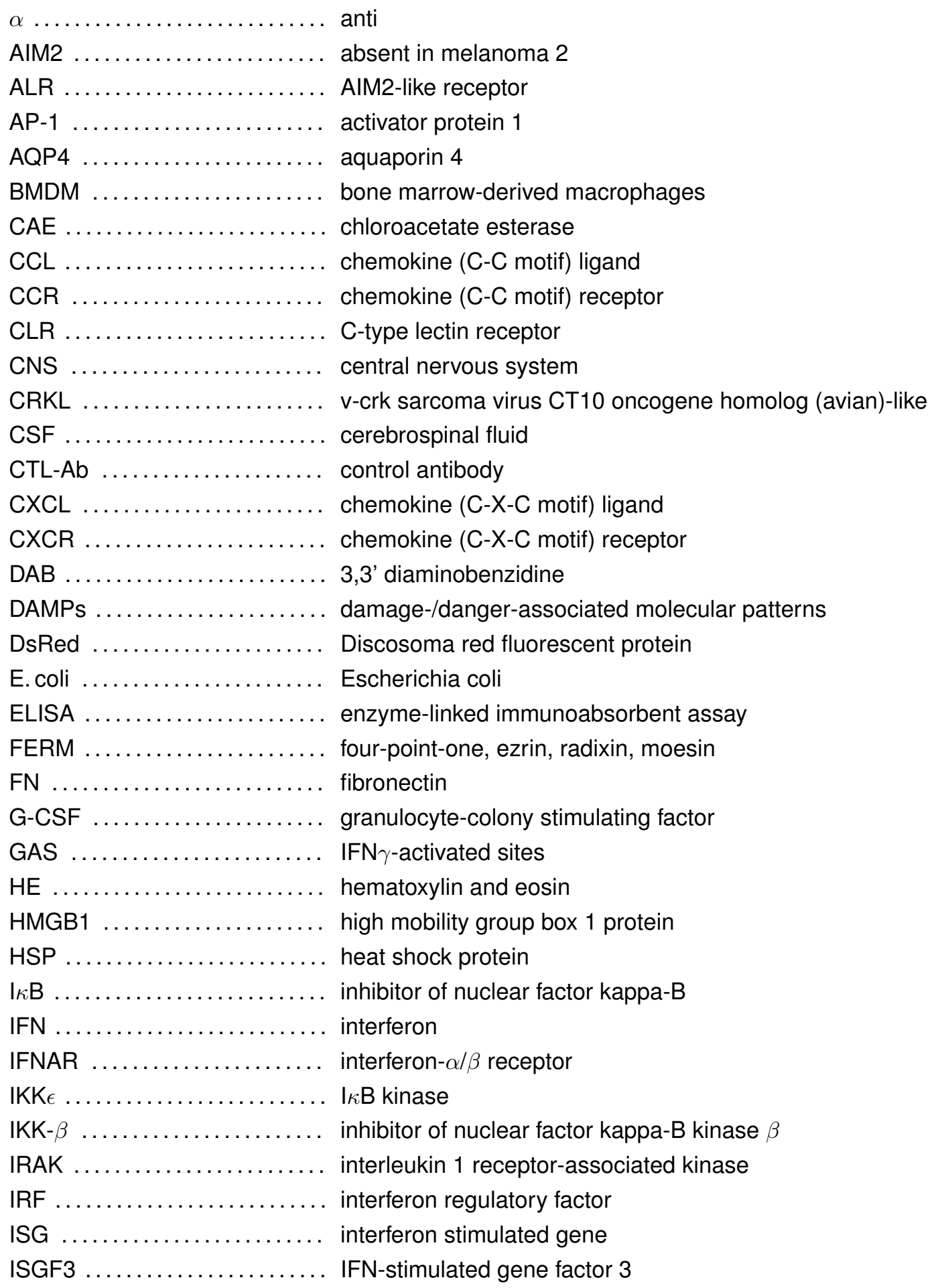




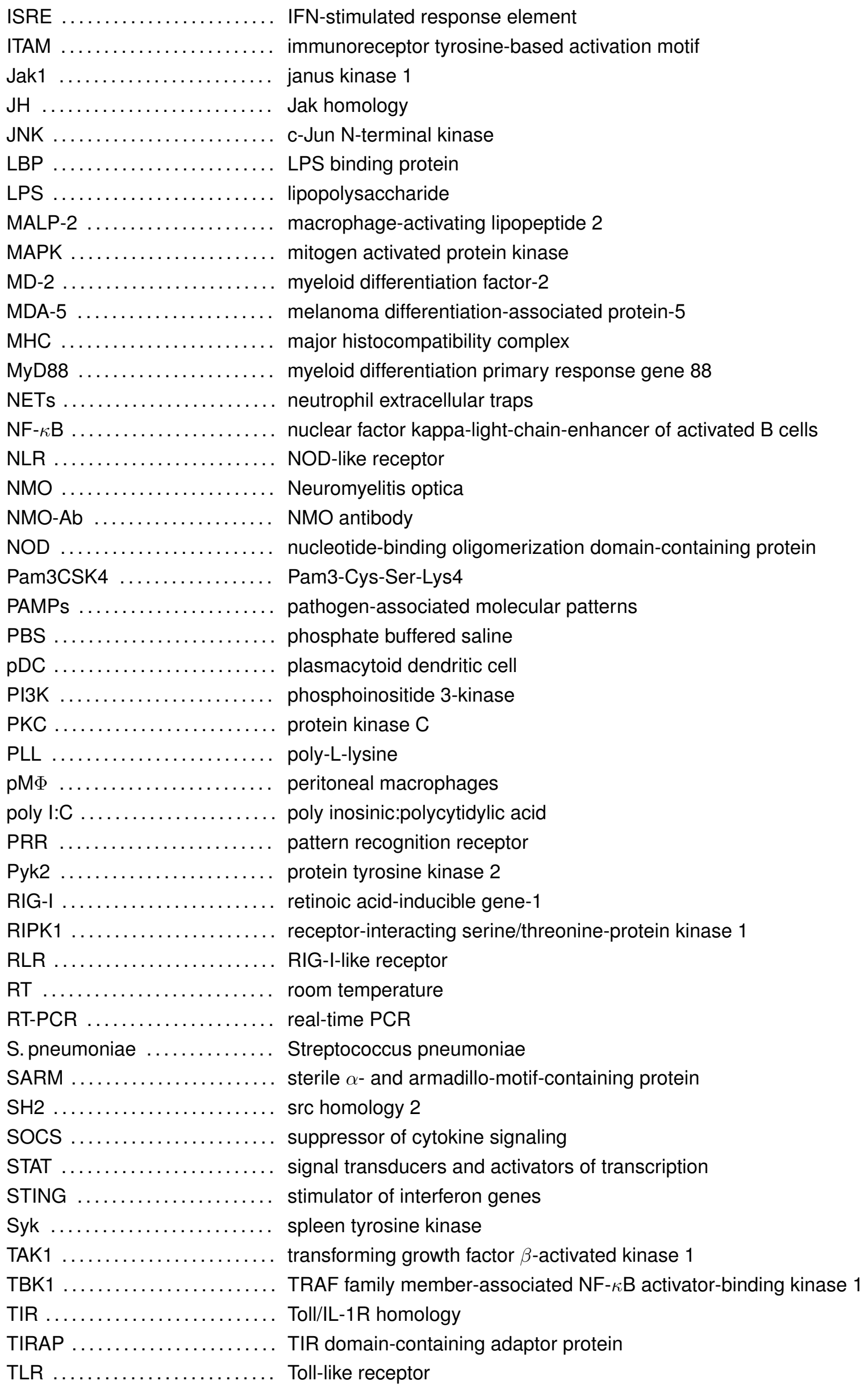


TNF

tumor necrosis factor

TRAF

TNF receptor associated factor

TRAM

TRIF-related adaptor molecule

TRIF

TIR domain-containing adaptor-inducing interferon- $\beta$

Tyk2 tyrosine kinase 2

USP18

ubiquitin specific peptidase 18

wt wildtype 


\section{Introduction}

\subsection{Inflammation and innate immunity}

The immune system is a complex biological system that exists to protect the host against a large variety of different diseases. In vertebrates, it can be classified into innate and adaptive immunity. The innate immunity enables a rapid response to several pathogens in a generic way. However, it lacks the ability of the adaptive immunity to specifically recognize a particular pathogen and provide long-lasting, protective memory for preventing reinfection. In response to harmful threats, such as infectious agents or tissue damage, inflammation is one of the first reactions of the immune system. The major functions of this complex process are elimination of the threat, clearance of the damaged tissue and initiation of tissue repair. It is part of the non-specific immune response mounted by the innate immune system.

The first line of defense against pathogens are anatomical barriers that protect from invading bacteria, viruses or parasites. Pathogens that overcome these barriers, immediately face tissue macrophages, which act as sentinel cells that initiate an inflammatory response. This is accompanied by the production of several soluble mediators of inflammation, such as vasoactive proteins, complement factors, cytokines, chemokines and lipid inflammatory mediators. As a first step, the diameter of surrounding blood vessels is increased leading to a slower blood flow. In combination with the induced expression of adhesion molecules this allows the attachment of leukocytes to the endothelium and their migration into the tissue. The last major change is an increase in blood vessel permeability resulting in exudation of fluid and plasma proteins into the tissue (Janeway et al. 2001).

Once inflammation has begun, neutrophils are the first cells rapidly attracted to the site of infection or damage. Neutrophils are the most abundant immune cell type and have a very short lifespan (Summers et al. 2010). They are produced in the bone marrow and released into the blood stream in response to inflammation. Their egress from the bone marrow and migration towards the site of inflammation is controlled by the granulocyte-colony stimulating factor (G-CSF) (Semerad et al. 2002) and chemokine (C-X-C motif) ligand (CXCL) 1 and CXCL2, the two ligands of chemokine (C-X-C motif) receptor (CXCR) 2 (Burdon et al. 2008; Wengner et al. 2008). The subsequent recruitment into the inflamed tissue involves the following steps: tethering, slow rolling, adhesion strengthening and spreading, intravascular crawling and paracellular or transcellular transmigration (Ley et al. 2007). Once neutrophils passed through the endothelium, they migrate towards the inflammatory site along chemotactic gradients and directly attack pathogens.

Neutrophils have different methods for pathogen elimination. After they encounter microorganisms, neutrophils phagocytose and destroy them intracellularly by NADPH oxygenase-dependent mechanisms or different antibacterial proteins, such as cathepsins, defensins, lactoferrin and lysozyme. These antibacterial proteins can also be released into the extracellular milieu in a process called degranulation in order to target the pathogens directly (Kolaczkowska and Kubes 2013). Furthermore, neutrophils can cause the release of neutrophil extracellular traps (NETs), which contain DNA and several proteins, such as 
neutrophil elastase, cathepsin G or lactoferrin. NETs associate with pathogens, preventing them from spreading and enabling their degradation through antimicrobial agents (Brinkmann et al. 2004).

In addition to fighting pathogens themselves, neutrophils recruit and activate other immune cells. Following their extravasation to the site of inflammation, they subsequently induce emigration of monocytes from the bone marrow (Soehnlein et al. 2009). Even though monocytes are also produced in the bone marrow, they are released as non-differentiated cells that circulate in the blood for 1-3 days (van Furth and Cohn 1968). Chemotactic gradients of chemokine (C-C motif) ligand (CCL) 2 and CCL7, which are recognized by the chemokine (C-C motif) receptor (CCR) 2, guide the monocytes to the inflamed tissue (Tsou et al. 2007). Monocyte extravasation is then realized in the same way as for neutrophils. Once they reach the tissue, they can differentiate into macrophages or myeloid dendritic cells (Warren and Vogel 1985; Zhou and Tedder 1996).

Monocytes and their progenies macrophages and dendritic cells serve important functions in fighting pathogens. They are able to phagocytose various materials and produce a large variety of inflammatory and immunoregulatory cytokines and chemokines (Kantari et al. 2008). Furthermore, they can present antigens on major histocompatibility complex (MHC) class II molecules in order initiate a response of the adaptive immune system specific to the respective antigens by activation of $T$ cells (Randolph et al. 2008).

According to the expression of distinct cell surface receptors, three subtypes of monocytes can be distinguished. In mice, these are classical Ly6C ${ }^{++} \mathrm{CD} 43^{+}$monocytes, intermediate Ly6C ${ }^{++} \mathrm{CD} 43^{++}$ monocytes and non-classical $\mathrm{Ly}_{6 \mathrm{C}} \mathrm{CD}^{+} \mathrm{C}^{++}$monocytes, which can be further characterized by their expression levels of CCR2 and $\mathrm{CX}_{3} \mathrm{CR} 1$ (Ziegler-Heitbrock et al. 2010). Classical murine monocytes are also referred to as inflammatory monocytes, because they are found in inflamed tissue, where they can trigger immune responses (Geissmann et al. 2003). In contrast to that, non-classical murine monocytes are also termed patrolling monocytes because of their ability to patrol along blood vessels in healthy tissue (Auffray et al. 2007) and under inflammatory conditions (Imhof et al. 2016). In humans, monocyte subtypes are classified according to the expression of CD14 and CD16 (Ziegler-Heitbrock et al. 2010).

After removal of the infectious agents by the immune cells, the inflammatory reaction needs to be terminated in order to protect from tissue damage and prevent chronicity. The resolution of inflammation includes anti-inflammatory and pro-resolving responses (Serhan 2010). Initiation of the termination is triggered by a switch in secretion of lipid mediators leading to retarded entry of new neutrophils to sites of inflammation (Serhan et al. 1995) and reduced vascular permeability (Takano et al. 1998). The neutrophils undergo apoptosis and are then phagocytosed by macrophages (Savill et al. 1989). Afterwards, macrophages egress from the inflamed tissue to the draining lymph nodes (Bellingan et al. 1996). Although the recruitment of monocytes and neutrophils is essential during infection, they might also have detrimental effects in unresolved, chronic inflammation. The production of reactive oxygen species, proteases and growth factors can lead to tissue destruction as well as excessive fibroblast proliferation and aberrant collagen accumulation resulting in fibrosis (Chen and Nuñez 2010). Therefore, inflammatory reactions need to be kept under tight control.

\subsection{Microglia as innate immune cells of the CNS}

Tissue-resident macrophages are an important part of the innate immune system. They serve several immune sentinel and homeostatic functions according to their specific location. Their diverse phe- 
notypes, which determine their pro- and anti-inflammatory functions, are mainly controlled by the respective tissue environment (Davies and Taylor 2015). There exist a lot of different tissue-resident macrophages, such as osteoclasts in the bone, Kupffer cells in the liver, alveolar macrophages in the lung, peritoneal macrophages in serosal tissues or Langerhans cells in the skin (Davies et al. 2013). Within the central nervous system (CNS), non-parenchymal perivascular, meningeal and choroid plexus macrophages as well as parenchymal microglia are present (Ransohoff and Cardona 2010).

Microglia are a type of glial cells that are generally considered as the immune cells of the CNS. According to their morphology, they have been first described as microglia by Pío del Río-Hortega in 1919 (del Río-Hortega 1919). Within the brain, microglia comprise 5-12\% of all cells, depending on their location (Lawson et al. 1990). In contrast to other macrophages, microglia do not derive from the definitive haematopoiesis, but from primitive myeloid progenitors that arise before embryonic day 8 in the yolk sac of mice (Ginhoux et al. 2010). This process is independent of the transcription factor Myb, which is important for the definitive haematopoiesis (Schulz et al. 2012), but rather depends on Pu.1 and interferon regulatory factor (IRF) 8 (Kierdorf et al. 2013). However, a minor non-yolk sac contribution to the origin of adult microglia was suggested recently (Xu et al. 2015). During adulthood, microglial numbers are sustained by local self-renewal (Ajami et al. 2007) through proliferation of local progenitor cells (Elmore et al. 2014).

The role of microglia in inflammatory conditions and neuropathological disorders was for a long time associated with detrimental actions and failure of protection. However, they also have many beneficial functions (Hanisch and Kettenmann 2007). Microglia are neuroprotective after ischemic injury (Neumann et al. 2006) and restrict the damage in acute brain injury by attenuating excitotoxicity (Simard and Rivest 2007). Furthermore, they provide growth factors that positively affect the efficiency of remyelination in a mouse model of toxic demyelination (Kotter et al. 2005). Most likely, excessive acute, chronic or maladaptive microglial responses exacerbate damage, while physiological responses are beneficial and protective (Hanisch and Kettenmann 2007).

Under homeostatic conditions, microglia constantly scan their environment in order to detect even slight disturbances. This constant tissue surveillance is realized by their extremely motile, highly ramified processes and protrusions that shape microglial morphology (Nimmerjahn et al. 2005). With their fine processes, microglia actively participate in remodeling synaptic circuits as they monitor the functional state of synapses and can lead to their modification or removal (Wake et al. 2009; Tremblay et al. 2010). During postnatal development, microglia also serve important functions in synaptic maturation as they actively engulf synaptic material and therefore are important for synaptic pruning (Paolicelli et al. 2011). Defective synaptic pruning has far-reaching consequences as its impairment can lead to decreased functional brain connectivity and therefore to deficits in social behavior (Zhan et al. 2014). Apart from affecting behavior (Chen et al. 2010), microglia also influence learning processes (Ziv et al. 2006; Parkhurst et al. 2013) and participate in oligodendrogenesis (Butovsky et al. 2006) and neurogenesis (Walton et al. 2006; Sierra et al. 2010).

Under pathological conditions, microglia can commit to distinct reactive phenotypes depending on the type of challenge. Sudden encounter or changes in a range of molecules can be interpreted as a sign of infection or injury (Hanisch and Kettenmann 2007). Shifting to an activated state goes along with drastic morphological changes that lead to a rounded, amoeboid shape of the cells (Kettenmann et al. 2011). These activated microglia can migrate to the site of infection or injury following chemotactic gradients of other microglia or immune cells (Yao et al. 1990; Honda et al. 2001). Once at the site 
of the inflammation, microglia execute diverse functions. As the tissue-resident macrophages of the CNS, they are able to phagocytose various materials. This includes infectious agents and endogenous material, such as pathological proteins, apoptotic cells and cellular debris (Garden and Möller 2006). Furthermore, microglia have been shown to participate in clearance of myelin, which plays a role in damaged CNS tissue and autoimmune diseases, but also as a house-keeping function (Fitzner et al. 2011). In addition to their phagocytic capacity, microglia are able to fight pathogens by generating nitric oxide and producing a respiratory burst (Banati et al. 1993). Apart from that, they secrete a large variety of other soluble factors, such as lipid inflammatory mediators (Minghetti and Levi 1998), cytokines and chemokines (Hanisch 2002). These factors have a broad range of autocrine and paracrine effects. The release of certain chemokines will attract additional microglia to the site of insult and recruit peripheral immune cells to the CNS, if necessary (Aloisi 2001). In order to mount an adaptive immune response, antigens need to be presented to $T$ cells. Therefore, microglia can act as antigen-presenting cells on demand by up-regulation of MHC class II and co-stimulatory molecules CD80 and CD86 (B7.1 and B7.2) (Hayes et al. 1987; Xu and Ling 1994). Overall, microglia do not only serve as immune effector cells themselves, but also coordinate the inflammatory response in the CNS.

\subsection{Toll-like receptors}

The innate immune system is capable of recognizing and rapidly responding to different types of threats. For that purpose, a variety of pattern recognition receptors (PRRs) is expressed by cells of the innate immunity. PRRs are germline-encoded receptors that evolved in order to detect pathogens by recognizing microbial conserved structural motifs, which are called pathogen-associated molecular patterns (PAMPs) (Takeuchi and Akira 2010). Furthermore, PRRs are also able to sense endogenous molecules that are linked to cell damage or death, called damage- or danger-associated molecular patterns (DAMPs) (Matzinger 1994; Matzinger 2002; Kono and Rock 2008).

There exist different classes of PRRs, including Toll-like receptors (TLRs), C-type lectin receptors (CLRs), retinoic acid-inducible gene-I (RIG-I)-like receptors (RLRs), nucleotide-binding oligomerization domain-containing protein (NOD)-like receptors (NLRs) and absent in melanoma 2 (AIM2)-like receptors (ALRs) (Brubaker et al. 2015).

Toll-like receptors are named after the Drosophila toll gene, which was discovered in 1985 (Anderson et al. 1985) and later on found to be involved in antifungal responses (Lemaitre et al. 1996). The human homologue of the Drosophila toll protein was identified in 1997 and shown to function as a receptor that controls the expression of several immune genes (Medzhitov et al. 1997).

To date, 10 TLRs have been described in humans (TLR1-10) and 12 in mice (TLR1-9 and TLR11-13) (Takeuchi and Akira 2010). They recognize distinct molecular patterns of which the most important ones are summarized in table 1.1. According to their respective ligand, the TLRs are differently localized within the cell (Takeuchi and Akira 2010). TLR1, TLR2, TLR4, TLR5, TLR6, TLR11 can be found at the cell surface, while TLR3, TLR7, TLR8, TLR9, TLR10, TLR12 (Raetz et al. 2013) and TLR13 (Shi et al. 2011) are located in intracellular compartments, such as endosomes. Upon ligand binding, the receptors dimerize and mostly form homodimers. However, several heterodimer combinations have been reported that lead to changes in ligand specificity. This includes TLR1/2 and TLR6/2 as classical examples (Kang and Lee 2011), but also TLR4/6 (Stewart et al. 2010) and TLR2/4 (Wang et al. 2014) heterodimers. 
TLR

\begin{tabular}{|c|c|c|}
\hline TLR1 & triacyl lipoprotein & bacteria \\
\hline TLR2 & $\begin{array}{l}\text { lipoprotein, HMGB1, HSPs, hyaluronan, } \\
\text { biglycan, versican }\end{array}$ & $\begin{array}{l}\text { bacteria, viruses, } \\
\text { parasites, self }\end{array}$ \\
\hline TLR3 & dsRNA & viruses \\
\hline TLR4 & $\begin{array}{l}\text { LPS, HMGB1, HSPs, hyaluronan, biglycan, } \\
\text { heparan sulfate, fibrinogen }{ }^{1} \text {, fibronectin }{ }^{2}\end{array}$ & bacteria, viruses, self \\
\hline TLR5 & flagellin & bacteria \\
\hline TLR6 & diacyl lipoprotein & bacteria, viruses \\
\hline TLR7 (human TLR8) & ssRNA & viruses, bacteria, self \\
\hline TLR9 & CpG-DNA, mitochondrial DNA & viruses, bacteria, protozoa, self \\
\hline TLR10 & unknown & unknown \\
\hline TLR11 & profilin-like molecule & protozoa \\
\hline TLR12 & profilin $^{3}$ & protozoa \\
\hline TLR13 & ribosomal RNA sequence ${ }^{4}$ & virus, bacteria \\
\hline
\end{tabular}

Table 1.1: TLRs and their ligands adapted from Takeuchi and Akira (2010) and Chen and Nuñez (2010) ${ }^{1}$ Smiley et al. (2001), ${ }^{2}$ Okamura et al. (2001), ${ }^{3}$ Koblansky et al. (2013), ${ }^{4}$ Oldenburg et al. (2012)

All TLRs are transmembrane glycoproteins that contain an extracellular domain with leucine-rich repeat motifs that is responsible for ligand binding, a transmembrane domain and an intracellular Toll/IL-1R homology (TIR) domain that is necessary for signal transduction (Brubaker et al. 2015). Activation of the receptor by ligand binding initiates the signaling cascade by recruitment of specific TIR domaincontaining adaptor proteins depending on the respective TLR. This family of proteins consists of myeloid differentiation primary response gene 88 (MyD88), TIR domain-containing adaptor protein (TIRAP), TIR domain-containing adaptor-inducing interferon- $\beta$ (TRIF) and TRIF-related adaptor molecule (TRAM) as well as sterile $\alpha$ - and armadillo-motif-containing protein (SARM) (O'Neill and Bowie 2007). In contrast to the other four family members, SARM does not activate, but negatively regulates TLR signaling (Carty et al. 2006).

A signaling pathway initiated by MyD88 is used by all TLRs, except TLR3. Following its association with the respective TLR, MyD88 recruits interleukin 1 receptor-associated kinase (IRAK) 4, IRAK1, IRAK2 and IRAK-M (Kawai and Akira 2010). While IRAK-M acts only as a negative regulator (Kobayashi et al. 2002), the other IRAKs participate in initiation of the TLR signaling. After the activation of IRAK4, IRAK1 and IRAK2 are activated consequently (Li et al. 2002; Kawagoe et al. 2008). The IRAKs then phosphorylate and thereby activate tumor necrosis factor (TNF) receptor associated factor (TRAF) 6. TRAF6 is an E3 ubiquitin ligase that together with an E2 ubiquitin-conjugating enzyme complex comprising Ubc13 and Uev1A catalyzes polyubiquitination, which activates the transforming growth factor $\beta$-activated kinase 1 (TAK1) complex (Wang et al. 2001). TAK1 then phosphorylates inhibitor of nuclear factor kappa-B (I $\kappa \mathrm{B})$ kinase $\beta$ (IKK- $\beta$ ), which results in nuclear factor kappa-B (NF- $\kappa \mathrm{B})$ activation. Furthermore, TAK1 phosphorylates the mitogen activated protein kinase (MAPK) kinases 6 and 7, which initiates the c-Jun N-terminal kinase (JNK) and p38 kinase pathway, followed by activation of activator 
protein 1 (AP-1) (Bhoj and Chen 2009). Finally, both transcription factors, NF- $\kappa$ B and AP-1, lead to the induction of inflammatory genes, such as cytokines and chemokines.

In addition to the MyD88-dependent pathway, TLR3 and TLR4 use the adaptor protein TRIF for their signaling. After recruitment of TRIF to the receptor, it activates the kinases receptor-interacting serine/ threonine-protein kinase 1 (RIPK1), TRAF family member-associated NF- $\kappa$ B activator-binding kinase 1 (TBK1) and $\mathrm{I}_{\kappa} \mathrm{B}$ kinase $\epsilon(\mathrm{IKK} \epsilon)$. RIPK1 causes a TRAF6/TAK1-dependent activation of NF- $\kappa$ B gene transcription as described above (Takeuchi and Akira 2010). Additionally, TBK1 and IKK $\epsilon$ phosphorylate IRF3, which results in the production of type I interferons and chemokines, such as CCL5 (Fitzgerald et al. 2003).

\subsubsection{TLR4}

TLR4, the first discovered TLR, was initially identified as the receptor recognizing lipopolysaccharide (LPS) (Poltorak et al. 1998; Hoshino et al. 1999). LPS is a cell wall component of gram-negative bacteria and comprises three different parts, lipid A, the core oligosaccharide and the O-antigen (also O-polysaccharide) (Beutler and Rietschel 2003). Based on their structure, LPS can be classified in different variants, also called chemotypes. Depending on the complexity of their carbohydrate moieties, smooth (S) and rough (R) LPS chemotypes can be distinguished (Raetz and Whitfield 2002). If LPS is liberated from bacteria during an infection, it associates with the LPS binding protein (LBP) in the plasma and is then transferred to the TLR4 co-receptor CD14 (Wright et al. 1990). CD14 extracts monomeric LPS molecules and presents them to a complex at the cell surface, consisting of TLR4 and myeloid differentiation factor-2 (MD-2) (Shimazu et al. 1999). Consequently, a dimeric TLR4/MD-2/LPS complex is formed (Park and Lee 2013).

Apart from recognizing LPS, TLR4 can respond to several DAMPs. This includes necrotic cell death related molecules, such as high mobility group box 1 protein (HMGB1) or heat shock proteins (HSPs), but also extracellular matrix fragments that are released during tissue injury, such as hyaluronan, biglycan or heparan sulfate (Kono and Rock 2008; Chen and Nuñez 2010). Extravascular fibrinogen and plasma fibronectin (FN), indicating vascular leakage, also activate TLR4 (Smiley et al. 2001; Okamura et al. 2001). These responses to DAMPs also require the co-receptor CD14. CD14 is necessary for responses to HMGB1 in murine macrophages (Kim et al. 2013) and HSP70 in human monocytes (Asea et al. 2000) as well as for reactions to tissue damage by murine microglia (Janova et al. 2016).

Following recognition of either PAMP or DAMP, the intracellular signaling cascade is triggered. In this regard, TLR4 is rather unique within the TLR family as it is the only TLR that uses both, the MyD88and the TRIF-dependent signaling pathway. This feature is enabled by the use of the two TIR domaincontaining adaptor proteins TIRAP and TRAM, which serve a sorting function. At first, the TIRAPMyD88 pathway is initiated at the plasma membrane and afterwards, the TLR4 complex is internalized in order to initiate the TRAM-TRIF pathway from endosomes (Kagan et al. 2008). Thereby, TLR4 stimulation leads to the production of MyD88-dependent cytokines and chemokines, but also to the TRIF-dependent production of type I interferons.

\subsubsection{TLR2}

TLR2 senses a variety of PAMPs derived from bacteria, fungi, parasites and viruses as well as several DAMPs (Akira et al. 2006). This ligand diversity is achieved by heterodimerization of TLR2 with TLR1 or TLR6, resulting in recognition of distinct ligands (Ozinsky et al. 2000). TLR1/2 heterodimers are activated by triacylated lipopeptides from gram-negative bacteria or mycoplasma and TLR6/2 heterodimers by diacylated lipopeptides from gram-positive bacteria or mycoplasma (Kawai and Akira 2010). As an 
example, TLR1/2 recognizes Pam3-Cys-Ser-Lys4 (Pam3CSK4), a synthetic, triacylated lipopeptide (Jin et al. 2007), and TLR6/2 recognizes macrophage-activating lipopeptide 2 (MALP-2) from Mycoplasma fermentans (Takeuchi et al. 2001).

Upon stimulation with one of these ligands, the MyD88-dependent signaling pathway is initiated by TIRAP as bridging molecule (Yamamoto et al. 2002). This culminates in the production of various proinflammatory cytokines. The TLR2-mediated activity can be enhanced in response to the gram-positive bacterial cell wall components lipoteichoic acid and peptidoglycan by the use of CD14 as co-receptor (Yoshimura et al. 1999) and the TLR2-induced NF- $\kappa$ B activation is also regulated by CD14 (Brandt et al. 2013). As the TRIF-dependent pathway is not triggered by TLR2 ligands, type I interferons are basically not produced in this scenario (Toshchakov et al. 2002). However, TLR2 is able to induce the production of type I interferons under certain circumstances in response to viruses (Barbalat et al. 2009) and some bacterial ligands (Dietrich et al. 2010). This signaling depends on MyD88 and requires, in addition to TIRAP, TRAM as a sorting adaptor protein, which activates IRF7 to induce type I interferon expression (Stack et al. 2014).

\subsection{Type I interferons}

Interferons have been discovered in 1957 and were named after their ability to interfere with influenza virus replication (Isaacs and Lindenmann 1957). These small molecules belong to the large class of proteins called cytokines (González-Navajas et al. 2012). Interferons are classified in three different types. The group of type I interferons consists of interferon (IFN) $\alpha, \operatorname{IFN} \beta, \operatorname{IFN} \epsilon, \operatorname{IFN} \kappa, \operatorname{IFN} \omega, \operatorname{IFN} \delta$, IFN $\tau$ and IFN $\zeta$ (Pestka 2007). Of these, IFN $\delta$ and IFN $\tau$ are neither present in humans nor in mice (Owens et al. 2014) and IFN $\zeta$ is only present in mice (Hardy et al. 2004). IFN $\epsilon$ is expressed only in the placenta and IFN $\kappa$ in keratinocytes (Theofilopoulos et al. 2005). Therefore, IFN $\alpha$ and IFN $\beta$ are the immunologically most relevant type I interferons. The IFN $\alpha$ family includes 13 subtypes in humans and 14 in mice, while there is only one IFN $\beta$ protein in both species (van Pesch et al. 2004). The group of type II interferons contains only IFN $\gamma$ and the group of type III interferons consists of IFN $\lambda 1$, IFN $\lambda 2$, IFN $\lambda 3$, also called IL-29, IL-28A and IL-28B, respectively (Sheppard et al. 2003; Kotenko et al. 2003).

Interferons are important signaling mediators within the immune system regulating both innate as well as adaptive immune responses. They are crucial for effectively fighting viral infections as they block the spread of virus particles by activating an antiviral state in infected and neighboring cells (Sen 2001; Samuel 2001). Furthermore, they increase the surface expression of MHC class I (David-Watine et al. 1990) and MHC class II molecules (Loughlin et al. 1993). This enhances the efficient recognition of infected cells by presentation of viral peptides and leads to their rapid elimination by activation of the immune system. Interferons exert antiproliferative as well as proapoptotic actions (Bekisz et al. 2010) and also stimulate the cytotoxic activity of different cell types, such as natural killer cells and monocytes (Ortaldo et al. 1983).

Type I interferons can be produced upon activation of several PRRs. Recognition of nucleic acids from viruses or bacteria by the intracellular TLRs (TLR3, TLR7 and TLR9) and also activation of TLR4 by LPS can induce the production of type I interferons (Kawai and Akira 2010). Furthermore, the RLRs RIG-I and melanoma differentiation-associated protein-5 (MDA-5) that sense viral RNA trigger type I interferons (Loo and Gale 2011). Apart from these, several other cytosolic PRRs can lead to type I interferon production. This includes members of the NLR family. NOD1 recognizes bacterial peptidoglycan moieties and triggers type I interferons in response to Helicobacter pylori infection (Watanabe et al. 2010). 
NOD2 is activated by the bacterial cell wall component muramyl dipeptide (Girardin et al. 2003) and is critical for the type I interferon response to Mycobacterium tuberculosis infections (Pandey et al. 2009). Additionally, the DNA-dependent activator of IFN (DAI) and the stimulator of interferon genes (STING) are activated by double-stranded DNA to induce type I interferons (Takaoka et al. 2007; Ishikawa et al. 2009). Furthermore, the ALR IFI16 also serves as intracellular microbial DNA sensor mediating IFN $\beta$ induction (Unterholzner et al. 2010). Alternatively, double-stranded DNA can be transcribed into RNA by the DNA-dependent RNA-polymerase III and subsequently activate RIG-I (Chiu et al. 2009).

Upon encounter with one of those stimuli, type I interferons can be produced by almost all cell types. The most potent producers of type I interferons are plasmacytoid dendritic cells (pDCs) (Fitzgerald-Bocarsly and Feng 2007). pDCs are able to produce up to 1,000-fold more interferons than other types of blood cells in response to viral infections (Siegal et al. 1999). Within the brain, pDCs are not present under physiological conditions. However, few numbers of cells can be found in the cerebrospinal fluid (CSF) from patients with non-inflammatory neurological diseases and these numbers are elevated during CNS inflammation (Pashenkov et al. 2001).

In the central nervous system, microglia and astrocytes are the main source of IFN $\beta$ (Kallfass et al. 2012; Costello and Lynch 2013). This correlates with the fact that both cell types are equipped with the necessary receptors. Microglia express all relevant TLRs (Kettenmann et al. 2011), RIG-I and MDA-5 (Furr et al. 2008) as well as DAl (Furr et al. 2011). Astrocytes express TLR3 at a high level (Bsibsi et al. 2002), while other TLRs, such as TLR4 and TLR9, are expressed at low levels (Bowman et al. 2003). Furthermore, astrocytes express RIG-I (Furr et al. 2010), MDA-5 (De Miranda et al. 2009) and DAI (Furr et al. 2011).

Upon activation of PRRs, e.g. by LPS or poly inosinic:polycytidylic acid (poly I:C), astrocytes are also able to produce IFN $\alpha$ (Carpentier et al. 2005). Microglial production of IFN $\alpha$ is mostly linked to neuropathological conditions, such as Alzheimer's disease (Yamada et al. 1994), but can also occur in response to Theiler's murine encephalomyelitis virus or LPS (Olson and Miller 2004).

\subsection{Type I interferon signaling}

All type I interferons share the same receptor, namely the interferon- $\alpha / \beta$ receptor (IFNAR). IFNAR is a cell surface receptor that consists of two subunits, IFNAR1 and IFNAR2 (Uzé et al. 1990; Colamonici and Domanski 1993). The presence of both subunits is required for effective ligand binding (Cohen et al. 1995). Each subunit is constitutively associated with a non-receptor tyrosine kinase of the janus kinase family. While IFNAR1 is associated with tyrosine kinase 2 (Tyk2) (Colamonici et al. 1994), IFNAR2 is associated with janus kinase 1 (Jak1) (Domanski et al. 1997). Upon ligand binding, the receptor dimerizes at the cell surface and a tyrosine phosphorylation cascade is initiated inside the cell. Jak1 and Tyk2 transphosphorylation occurs starting with Jak1 phosphorylating Tyk2, which then cross-phosphorylates Jak1 for further activation (Stark et al. 1998). Subsequently, the activated kinases phosphorylate conserved tyrosine residues in the cytoplasmic tail of the receptor (Hervas-Stubbs et al. 2011). These serve as docking site for the src homology $2\left(\mathrm{SH}_{2}\right)$ domain of signal transducers and activators of transcription (STAT) proteins (Heim et al. 1995; Yan et al. 1996). Following their recruitment to IFNAR, several of these STAT proteins become tyrosine phosphorylated and thereby activated. This includes STAT1 and STAT2 as the most important mediators of type I interferon responses, but also STAT3 and STAT5 (Platanias 2005). However, STAT1 needs the presence of already phosphorylated STAT2 for its phosphorylation, while STAT2 can be phosphorylated on its own (Leung et al. 1995). In addition to that, IFN $\alpha$ induces the phosphorylation of STAT4 and STAT6 in certain cell types, such 
as endothelial or lymphoid cells (Fasler-Kan et al. 1998; Farrar et al. 2000; Torpey et al. 2004). If STAT1 and STAT2 become activated, they form a heterodimer, recruit IRF9 and translocate into the nucleus. This STAT1-STAT2-IRF9 complex is called IFN-stimulated gene factor 3 (ISGF3) complex (Martinez-Moczygemba et al. 1997). The ISGF3 complex binds to IFN-stimulated response elements (ISREs) in the promoter of IFN-stimulated genes (ISGs) and thereby regulates transcription (Decker et al. 2005). Even though ISGF3 is the most important transcription factor for type I interferon responses, also other activated STATs can either form homodimers (STAT1, STAT3, STAT4, STAT5 and STAT6) or heterodimers (STAT1/3, STAT1/4, STAT1/5, STAT2/3 and STAT5/6) that translocate into the nucleus and bind to IFN $\gamma$-activated sites (GAS) (Hervas-Stubbs et al. 2011). STAT1/2 heterodimers are also able to bind to GAS, if they are not associated with IRF9 (Li et al. 1996).

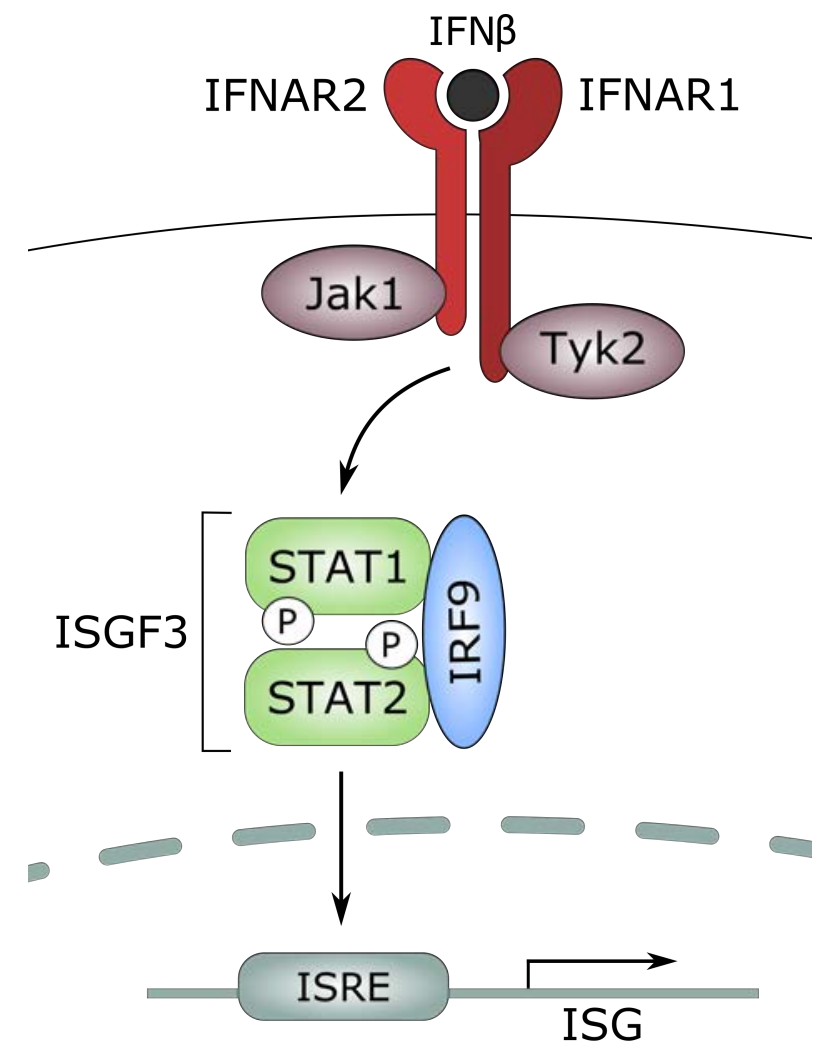

Figure 1.1: The canonical type I interferon signaling pathway Type I interferons, here represented by IFN $\beta$, are recognized by the cell surface receptor IFNAR. This receptor consist of the two subunits IFNAR1 and IFNAR2 that are both constitutively associated with a non-receptor tyrosine kinase. While IFNAR1 is associated with Tyk2, IFNAR2 is associated with Jak1. Following binding of IFN $\beta$, the receptor dimerizes and both tyrosine kinase become activated by transphosphorylation. Subsequently, they phosphorylate conserved tyrosine residues in the cytoplasmic tail of the receptor, which provides a docking site for STAT1 and STAT2. These transcription factors become also phosphorylated and are then able to form a heterodimer. This STAT1/STAT2 heterodimer recruits IRF9 to form the ISGF3 complex and then translocates into the nucleus, where it binds to ISREs in the promoter region of ISGs and thereby regulates transcription. Adapted from Ivashkiv and Donlin (2014).

Apart from the canonical type I interferon signaling, different other signaling pathways can be activated and contribute to type I interferon responses. This includes the v-crk sarcoma virus CT10 oncogene homolog (avian)-like (CRKL) pathway, the MAPK pathway, the phosphoinositide 3-kinase (PI3K) pathway and either the classical or the alternative NF- $\kappa$ B cascade (Hervas-Stubbs et al. 2011).

Type I interferon responses are tightly regulated in order to adjust the biological outcome. In general, the components of the canonical type I interferon signaling pathway are constitutively expressed in most cell types meaning that, in principle, they are able to respond to type I interferons. Immune cells can mount very rapid responses to even low levels of type I interferons. This is based on an autocrine loop initiated 
by small amounts of IFN $\beta$ that leads to high basal expression levels of STAT1 and IRF9 (Gough et al. 2012). In general, the regulation of STAT1 and IRF9 expression is an important mechanism to control type I interferon signaling. Because both proteins are ISGs, they can be induced by type I interferons, IFN $\gamma$ and other cytokines that activate STATs. This provides a basal level of antiviral protection even in the absence of ongoing signaling (Cheon et al. 2013). Apart from its expression, also the transcriptional activity of STAT1 can be modified. On the one hand, it can be enhanced through STAT1 phosphorylation at a conserved carboxy-terminal serine by several kinases of the protein kinase C (PKC) and MAPK family (Uddin et al. 2002; Goh et al. 1999). On the other hand, STAT tyrosine phosphorylation can be augmented by immunoreceptor tyrosine-based activation motif (ITAM) signaling utilizing spleen tyrosine kinase (Syk) and protein tyrosine kinase 2 (Pyk2) (Wang et al. 2008). An important mechanism to suppress type I interferon signaling is the downregulation of IFNAR surface levels. This can be achieved by several mechanisms, including inflammatory signaling or metabolic stress (Huangfu et al. 2012; Bhattacharya et al. 2013). Additionally, negative regulators, such as suppressor of cytokine signaling (SOCS) and ubiquitin specific peptidase 18 (USP18), influence type I interferon signaling (Krebs and Hilton 2001; Sarasin-Filipowicz et al. 2009). While SOCS proteins compete with STATs for IFNAR binding and suppress enzymatic activity of janus kinases (Croker et al. 2008), USP18 displaces Jak1 from IFNAR2 (Malakhova et al. 2006). Further suppressive mechanisms include the pausing of RNA polymerase II at IFN pathway genes and the induction of several microRNAs (Ivashkiv and Donlin 2014).

\subsection{Protective and detrimental roles in the CNS}

\subsubsection{Dual role of TLRs}

Within the CNS, TLRs are expressed on various cell types (see table 1.2). They are important for fighting pathogens and responding to tissue damage. However, as their activation leads to initiation of inflammatory responses, they are likewise associated with neurotoxicity.

\begin{tabular}{cc} 
Cell type & TLR expression \\
\hline microglia & TLR1, 2, 3, 4, 5, 6, 7, 8, 9, 11 ${ }^{1}, 13^{2}$ \\
\hline astrocytes & TLR1, 2, 3, 4, 5, 6, 9, 11 ${ }^{1}, 13^{2}$ \\
\hline oligodendrocytes & TLR2, 3 \\
\hline neurons & TLR3, 11 ${ }^{1}, 12^{2}, 13^{2}$ \\
Table 1.2: TLR expression in the CNS adapted from Hanisch et al. (2008) \\
${ }^{1}$ Atmaca et al. (2014), ${ }^{2}$ Mishra et al. (2008)
\end{tabular}

Destructive effects of TLRs were shown, for instance, by activation of TLR2 leading to inflammation and associated neuron loss (Hoffmann et al. 2007). In this case, toxicity appears to be primarily mediated by nitric oxide production resulting in neuronal apoptosis (Lehnardt et al. 2006). Furthermore, TLRs are involved in detrimental outcomes of brain ischemia and ischemic stroke. TLR2 was shown to propagate stroke-induced CNS injury (Lehnardt et al. 2007) and similar results were obtained for TLR4 as its absence reduces infarct size and the inflammatory response after an ischemic insult (Caso et al. 2007). Additionally, LPS-induced TLR4 responses lead to severe axonal and neuronal loss in a model of a normally subthreshold hypoxic ischemia (Lehnardt et al. 2003). The harmful effects of TLR4 responses were also shown by local injections of LPS into the CNS, which result in severe loss of dopamine neurons in the substantia nigra (Castaño et al. 1998) and neurons in the hippocampus 
(Hauss-Wegrzyniak et al. 1998). Apart from causing neuronal apoptosis, activation of TLR4 by LPS induces loss of oligodendrocytes and therefore hypomyelination (Lehnardt et al. 2002). This could have implications for demyelinating disorders, such as multiple sclerosis. In the brains of multiple sclerosis (Bsibsi et al. 2002) as well as Alzheimer's disease patients (Jackson et al. 2006) elevated TLR3 expression was detected, indicating an involvement of TLRs in neurodegeneration. Neuropathic pain seems to be also modulated by TLRs. TLR4 plays an important role in the induction of behavioral hypersensitivity in rodent models of neuropathy (Tanga et al. 2005) and TLR2 contributes to nerve injury-induced spinal cord glial cell activation with subsequent pain hypersensitivity (Kim et al. 2007). In addition to that, TLR activation in response to pathogens is also not always protective in the CNS. Lethality of West Nile virus and Herpes simplex virus 1 infection that cause encephalitis is mediated by TLR3 (Wang et al. 2004) and TLR2 (Kurt-Jones et al. 2004), respectively. Therefore, TLRs seem to have a dual role in CNS inflammation.

In responses to several bacterial infections, the presence of TLRs is beneficial and necessary for properly fighting the pathogens. In the absence of TLR2, the severity of Streptococcus pneumoniae (S. pneumoniae) meningitis is enhanced (Echchannaoui et al. 2002; Koedel et al. 2003) and $t / r 2^{-/}$mice were shown to be highly susceptible to Staphylococcus aureus infection (Takeuchi et al. 2000). Furthermore, TLR2 and TLR4 are required for an optimal intracerebral immune response in Staphylococcus aureus-induced brain abscess (Stenzel et al. 2008). Still, TLR-triggered inflammatory responses need to be properly controlled in order to limit harmful outcomes. A simple way to terminate TLR signaling is the clearance and degradation of the activation stimulus. This can be achieved by phagocytosis, a process that can be enhanced by TLR activity. Bacterial uptake and intracellular killing by microglia are increased after application of TLR agonists (Ribes et al. 2010). Similar results were obtained for amyloid $\beta$ uptake and clearance, indicating a beneficial role in Alzheimer's disease (Tahara et al. 2006; Chen et al. 2006). In addition to removal of infectious and damage-related agents, several neuroprotective mechanisms are favored by TLRs. In a model of spinal cord injury, TLR2 and TLR4 positively affect the functional recovery (Kigerl et al. 2007). Furthermore, TLR4 can protect the brain tissue from damage by toxic compounds (Glezer et al. 2006). Overall, TLR-mediated responses do not only have detrimental outcomes, but rather favor neuroprotection.

\subsubsection{Dual role of interferons}

Type I interferons are tightly linked to TLR signaling. Their production is induced by activation of TLR3, TLR7, TLR9 and TLR4 as well as, under certain circumstances, by TLR2. In the CNS, glial cells, mostly microglia and astrocytes, (Owens et al. 2014) as well as neurons (Préhaud et al. 2005; Delhaye et al. 2006) produce type I interferons in response to innate immune stimulation. Although they were mainly appreciated for their antiviral functions, type I interferons are also involved in other immunological responses.

In bacterial infections, type I interferons have opposing roles. On the one hand, they participate in the resistance against bacteria. This includes Chlamydia species (de la Maza et al. 1985; Buss et al. 2010), Legionella pneumophila (Plumlee et al. 2009) as well as group B streptococci, S. pneumoniae and Escherichia coli (E. coli) (Mancuso et al. 2007). On the other hand, type I interferon production is associated with suppression of responses to bacterial infections. IFNAR1-deficient mice are more resistant to Listeria monocytogenes infections (Auerbuch et al. 2004) and have a decreased and late mortality in Mycobacterium tuberculosis infection (Manca et al. 2005). Connected to that, type I interferons are essential effectors in gram-negative sepsis leading to LPS-induced lethality by endotoxic shock 
(Karaghiosoff et al. 2003).

However, type I interferons also play a role in non-infectious inflammatory conditions. Several diseases are associated with high levels of type I interferons. In Aicardi-Goutierès syndrome, which is a rare, early onset childhood, genetic disease with severe neurological dysfunction, overexpression of IFN $\alpha$ is present. This is caused by mutations in any one of several genes leading to impaired clearance of endogenous nucleic acids, which in turn trigger PRRs to overproduce IFN $\alpha$ (Crow and Rehwinkel 2009). Similar to that, the disease severity of systemic lupus erythematosus is closely linked to IFN $\alpha$ production (Kirou et al. 2005) and for the pathogenesis of neuropsychiatric systemic lupus erythematosus, IFN $\alpha$ could be even causative (Campbell et al. 1999; Santer et al. 2009). In HIV-infected patients suffering from dementia, IFN $\alpha$ levels are also elevated in the CSF compared to patients without dementia or controls and these increased IFN $\alpha$ levels correlate with neurocognitive dysfunctions (Rho et al. 1995). Furthermore, a mouse model of amyotrophic lateral sclerosis revealed a link between astrocytic IFN $\beta$ production and pathological changes in motor neurons (Wang et al. 2011).

Interestingly, effects of type I interferons are highly disease-specific. IFN $\beta$ is beneficial and used as therapeutic in multiple sclerosis (The IFNB Multiple Sclerosis Study Group 1993). However, in neuromyelitis optica, another demyelinating disease of the CNS, IFN $\beta$ treatment is harmful and increases autoantibody titers (Palace et al. 2010) as well as relapse rates (Kim et al. 2012).

Protective effects of type I interferons refer, at least in part, to their ability to control homeostatic and pathologic processes. The integrity of the blood-brain barrier is stabilized by IFN $\beta$ (Kraus et al. 2004), which protects against ischemic injury through actions on endothelial cells (Gesuete et al. 2012). Furthermore, type I interferons control immune cell infiltration into the brain, thereby preventing excessive inflammatory responses in the CNS. In the absence of essential parts of type I interferon signaling, leukocyte infiltration into the brain is increased in a model of sterile injury (Khorooshi and Owens 2010) and also a model of multiple sclerosis (Galligan et al. 2010). Thus, type I interferons could prove to be key regulators of CNS infection and damage. 


\section{Aim of the study}

Interferons are pleiotropic cytokines that were initially described as crucial mediators of host defense against viral infections. More recently, they were also recognized for their potential to fight bacterial infections. Apart from that, increasing evidence demonstrates also regulatory effects of interferons on innate and adaptive immune responses under physiological and pathological conditions. Especially these immunomodulatory functions could prove to be crucial for maintaining homeostasis and determining the outcome of different diseases. Since type I interferons represent the largest subgroup of these proteins with a huge variety of effects, they are of special interest for shaping immune reactions in diverse contexts. Indeed, type I interferons were already shown to be involved in the pathogenesis of several disorders and to even increase disease severity. In contrast to that, they also exert beneficial effects and are approved as treatment for individual diseases. However, exact mechanisms of their broad range of immunological activities are still poorly understood.

Within the CNS, microglia are the principle innate immune cells that serve important functions in health and disease. They are capable of sensing infections and damage through diverse receptors, such as TLRs, and consequently trigger an appropriate immune response. Upon activation by several stimuli, they can produce different interferons with distinct effects on other cells. Interestingly, some microglial functions are also regulated by interferons. This dual relationship of on the one hand being producers and on the other hand target of interferons makes these cells ideal candidates for investigating functional mechanisms of these immunomodulatory molecules. According to that, the following questions were addressed in this thesis:

\section{Under which conditions do microglia produce type I interferons?}

- Which type I interferons can be produced in response to activation of different TLRs?

- Which signaling pathways control this production?

\section{How are microglial responses to CNS infection and damage regulated by type I interferons?}

- How is the production of TLR-induced chemokines regulated by type I interferons?

- Does this regulation involve the canonical type I interferon signaling pathway?

- Do type I interferons similarly regulate responses of microglia and extraneural macrophage populations?

- Are effects of type I interferon signaling on different TLR systems individually organized?

- Is the outcome of selected infectious diseases and autoimmune disorders of the CNS modulated by type I interferon signaling? 


\section{Material and Methods}

\subsection{Material}

\subsubsection{Reagents}

Table 3.1: Reagents for in vitro stimulation of cells

\begin{tabular}{ll} 
Reagent & Source of supply \\
\hline E. coli DsRed DH5 $\alpha$ & $\begin{array}{l}\text { Dr. S. Ribes, Institute for Neuropathology, University Medi- } \\
\text { cal Center Göttingen, Germany }\end{array}$ \\
\hline IFN $\beta$ & PBL Assay Science, Piscataway, NJ, USA \\
\hline MALP-2 & Enzo Life Sciences/Alexis, Lörrach, Germany \\
\hline mouse fibronectin & Molecular Innovations, Novi, MI, USA \\
\hline Pam3CSK4 & Enzo Life Sciences/Alexis, Lörrach, Germany \\
\hline poly I:C & Enzo Life Sciences/Alexis, Lörrach, Germany \\
\hline $\begin{array}{l}\text { Re-LPS (rough chemotype } \\
\text { E. coli, serotype R515) }\end{array}$ & Enzo Life Sciences/Alexis, Lörrach, Germany \\
\hline $\begin{array}{l}\text { S-LPS (smooth chemotype } \\
\text { E. coli, serotype O55:B5) }\end{array}$ & EPS,
\end{tabular}

Table 3.2: Reagents

\begin{tabular}{ll} 
Reagent & Source of supply \\
\hline Ampicillin & Sigma-Aldrich Chemie GmbH, Steinheim, Germany \\
\hline Aquamount & Thermo Fisher Scientific, Waltham, MA, USA \\
\hline$\beta$-mercaptoethanol & Sigma-Aldrich Chemie GmbH, Steinheim, Germany \\
\hline Chloralhydrate & Merck Millipore, Darmstadt, Germany \\
\hline DAB & Sigma-Aldrich Chemie GmbH, Steinheim, Germany \\
\hline DePeX medium & VWR international, Darmstadt, Germany \\
\hline DMEM & Gibco, Karlsruhe, Germany \\
\hline DNase & CellSystem, St. Katherine, Switzerland \\
\hline
\end{tabular}




\begin{tabular}{|c|c|}
\hline EDTA & Roth, Karlsruhe, Germany \\
\hline Eosin G & Merck Millipore, Darmstadt, Germany \\
\hline FCS & Gibco, Karlsruhe, Germany \\
\hline Gentamicin & Sigma-Aldrich Chemie GmbH, Steinheim, Germany \\
\hline HBSS & Biochrom, Berlin, Germany \\
\hline Horse serum & Sigma-Aldrich Chemie GmbH, Steinheim, Germany \\
\hline Hydrogen peroxide, $30 \%$ solution & Merck Millipore, Darmstadt, Germany \\
\hline Ketamine & Medistar, Hannover, Germany \\
\hline Lysis buffer & Cell Signaling Technology, Cambridge, UK \\
\hline Mayer's hemalum solution & Merck Millipore, Darmstadt, Germany \\
\hline Monastral Blue & Sigma-Aldrich Chemie GmbH, Steinheim, Germany \\
\hline Paraffin (Paraplast Plus®) & Tyco Healthcare, Neustadt, Germany \\
\hline PBS & Gibco, Karlsruhe, Germany \\
\hline Penicillin & Gibco, Karlsruhe, Germany \\
\hline Percoll & GE Healthcare Life Sciences, Freiburg, Germany \\
\hline PFA & Merck Millipore, Darmstadt, Germany \\
\hline PLL & Gibco, Karlsruhe, Germany \\
\hline PMSF & Sigma-Aldrich Chemie GmbH, Steinheim, Germany \\
\hline Sodium pyruvate & Sigma-Aldrich Chemie GmbH, Steinheim, Germany \\
\hline Streptavidin horseradish peroxidase & Sigma-Aldrich Chemie GmbH, Steinheim, Germany \\
\hline Streptomycin & Gibco, Karlsruhe, Germany \\
\hline Trypsin & Biochrom, Berlin, Germany \\
\hline Trypsin/EDTA & Biochrom, Berlin, Germany \\
\hline WST-1 & Roche Applied Science, Mannheim, Germany \\
\hline Xylazine & Riemser Arzneimittel AG, Greifswald, Germany \\
\hline
\end{tabular}

Table 3.3: Kinase inhibitors

\begin{tabular}{llll} 
Inhibitor & Target & IC50 & Source of supply \\
\hline GLPG0634 & Jak1 & $10 \mathrm{nM}$ & Hölzel diagnostika, Köln, Germany \\
& Tyk2 & $116 \mathrm{nM}$ & \\
\hline Jak inhibitor I & Jak1 & $15 \mathrm{nM}$ & Calbiochem/Merck Millipore, Nottingham, UK \\
& Tyk2 & $1 \mathrm{nM}$ & \\
\hline Solcitinib & Jak1 & N/A & Hölzel diagnostika, Köln, Germany
\end{tabular}




\subsubsection{Buffers, solutions and cell culture media}

Table 3.4: Buffers and solutions

\begin{tabular}{|c|c|}
\hline Solution & Composition \\
\hline \multirow[t]{2}{*}{ Blocking buffer } & PBS \\
\hline & $10 \%$ FCS \\
\hline \multirow[t]{3}{*}{ DAB solution } & PBS \\
\hline & $0.5 \mathrm{mg} / \mathrm{mL} \mathrm{DAB}$ \\
\hline & $\begin{array}{l}20 \mu \mathrm{L} 30 \% \text { hydrogen peroxide added to } 50 \mathrm{~mL} \text { DAB so- } \\
\text { lution before use }\end{array}$ \\
\hline \multirow[t]{4}{*}{ FACS buffer } & PBS \\
\hline & $2 \%$ FCS \\
\hline & $0.01 \mathrm{M}$ EDTA, $\mathrm{pH}=8.0$ \\
\hline & $0.1 \% \mathrm{NaN3}$ \\
\hline \multirow[t]{3}{*}{$4 \%$ PFA solution } & PBS \\
\hline & $4 \%$ PFA \\
\hline & $\mathrm{pH}=7.4$ \\
\hline \multirow[t]{2}{*}{$1 \% \mathrm{HCl}$-alcohol } & $70 \%$ ethanol \\
\hline & $1 \% \mathrm{HCl}$ absolute \\
\hline \multirow[t]{4}{*}{$1 \%$ eosin solution } & $70 \%$ isopropylalcohol \\
\hline & $1 \%$ eosin $G$ \\
\hline & stirred and filtered \\
\hline & $0.5 \%$ acetic acid added before use \\
\hline \multirow[t]{2}{*}{$3 \%$ hydrogen peroxide solution } & PBS \\
\hline & $3 \%$ hydrogen peroxide \\
\hline
\end{tabular}

Table 3.5: Cell culture media

Solution

Complete culture medium

\section{Composition}

Dulbecco's modified Eagle medium (DMEM)

$10 \%$ FCS (heat inactivated for $30 \mathrm{~min}$ at $56^{\circ} \mathrm{C}$ )

$100 \mathrm{U} / \mathrm{mL}$ penicillin

$100 \mu \mathrm{g} / \mathrm{mL}$ streptomycin

Pluznik medium

\section{DMEM}

$10 \%$ FCS (heat inactivated for $30 \mathrm{~min}$ at $56^{\circ} \mathrm{C}$ )

$5 \%$ horse serum

30\% L929-conditioned medium

$1 \%$ sodium pyruvate

$100 \mathrm{U} / \mathrm{mL}$ penicillin

$100 \mu \mathrm{g} / \mathrm{mL}$ streptomycin

$50 \mathrm{mM} \beta$-mercaptoethanol 


\subsubsection{Antibodies}

Table 3.6: Blocking antibodies

\begin{tabular}{lll} 
Specificity & Clone & Source of supply \\
\hline IFNAR1 & MAR1-5A3 & BioLegend, London, UK \\
\hline IFNAR2 & polyclonal goat IgG & R\&D systems, Wiesbaden, Germany
\end{tabular}

Table 3.7: Antibodies for flow cytometry

\begin{tabular}{lllll} 
Specificity & Fluorochrome & Clone & Dilution & Source of supply \\
\hline CD11b & APC & M1/70 & $1: 100$ & BioLegend, London, UK \\
\hline CD11b & FITC & M1/70 & $1: 100$ & BioLegend, London, UK \\
\hline CD16/CD32 & & $2.4 G 2$ & $1: 50 / 1: 100$ & BD Biosciences, Franklin Lakes, NJ, USA \\
\hline CD3 & PE & 145-2C11 & $1: 100$ & BioLegend, London, UK \\
\hline CD45 & PerCP-Cy5.5 & 30-F11 & $1: 100$ & BioLegend, London, UK \\
\hline Ly6C & APC & HK1.4 & $1: 100$ & BioLegend, London, UK \\
\hline Ly6G & Pacific Blue & 1A8 & $1: 67$ & BioLegend, London, UK
\end{tabular}

Table 3.8: Antibodies for induction of focal NMO-like lesions

\begin{tabular}{lll} 
Specificity & Clone & Source of supply \\
\hline AQP4 & rAB-53 & $\begin{array}{l}\text { Dr. J. Bennett, Department of Neurology and Oph- } \\
\text { thalmology, University of Colorado, Denver, USA }\end{array}$ \\
\hline Measles virus nucleocapsid protein & rAb-2B4 & $\begin{array}{l}\text { Dr. J. Bennett, Department of Neurology and Oph- } \\
\text { thalmology, University of Colorado, Denver, USA }\end{array}$
\end{tabular}

Table 3.9: Immunohistochemistry antibodies

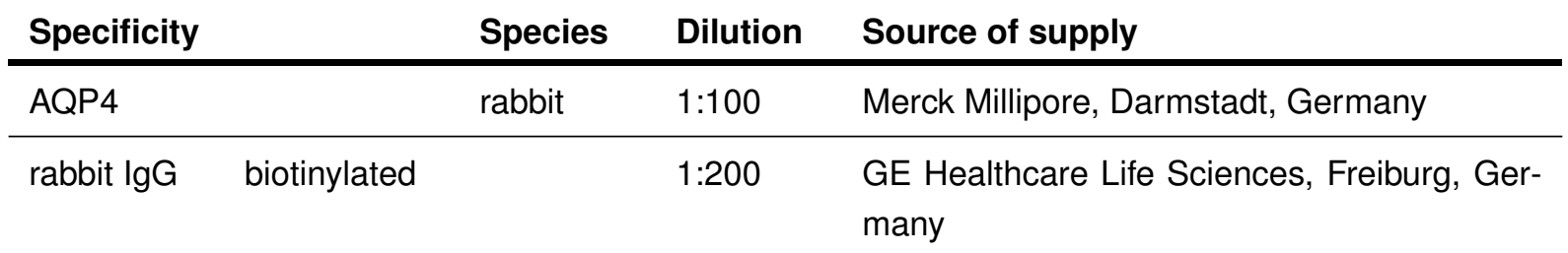




\subsubsection{Applied kits}

Table 3.10: Applied kits

\begin{tabular}{|c|c|}
\hline Kit & Source of supply \\
\hline DyLight $^{\mathrm{TM}} 550$ Labeling Kit & Thermo Fisher Scientific, Waltham, MA, USA \\
\hline $\mathrm{iTaq}^{\mathrm{TM}}$ Universal SYBR ${ }^{\circledR}$ Green Supermix & Bio-Rad, Hercules, CA, USA \\
\hline LEGEND MAX ${ }^{\mathrm{TM}}$ Mouse IFN $\beta$ ELISA Kit & BioLegend, London, UK \\
\hline Mouse CXCL1/KC DuoSet ELISA & R\&D Systems, Wiesbaden, Germany \\
\hline Mouse MCP-1 ELISA MAX ${ }^{\mathrm{TM}}$ Standard & BioLegend, London, UK \\
\hline $\begin{array}{l}\text { Naphthol AS-D chloroacetate (specific es- } \\
\text { terase) kit }\end{array}$ & $\begin{array}{l}\text { Sigma-Aldrich Chemie } \mathrm{GmbH} \text {, Steinheim, } \\
\text { Germany }\end{array}$ \\
\hline Neural Tissue Dissociation Kit $(\mathrm{T})$ & Miltenyi Biotec, Bergisch Gladbach, Germany \\
\hline QuantiTect ${ }^{\circledR}$ Reverse Transcription Kit & Qiagen, Hilden, Germany \\
\hline RNeasy Mini Kit & Qiagen, Hilden, Germany \\
\hline $\mathrm{RT}^{2}$ First Strand Kit & Qiagen, Hilden, Germany \\
\hline $\mathrm{RT}^{2}$ Profiler PCR Array & Qiagen, Hilden, Germany \\
\hline VeriKine Mouse IFN $\alpha$ ELISA Kit & PBL Assay Science, Piscataway, NJ, USA \\
\hline
\end{tabular}

\subsubsection{Primer for real-time PCR}

Table 3.11: Primer for real-time PCR

\begin{tabular}{lll} 
Gene & Unique assay ID & Source of supply \\
\hline ifnab & qMmuCED0004300 & Bio-Rad, Hercules, CA, USA \\
\hline ifnb1 & qMmuCED0050444 & Bio-Rad, Hercules, CA, USA \\
\hline stat1 & qMmuCED0046088 & Bio-Rad, Hercules, CA, USA \\
\hline stat2 & qMmuCED0048821 & Bio-Rad, Hercules, CA, USA \\
\hline stat3 & qMmuCED0044698 & Bio-Rad, Hercules, CA, USA
\end{tabular}




\subsubsection{Consumables}

Table 3.12: Consumables

\begin{tabular}{|c|c|}
\hline Consumable & Source of supply \\
\hline Blaubrand ${ }^{\circledR}$ intraMARK micropipettes, $5 \mu \mathrm{L}$ & Brand GmbH, Wertheim, Germany \\
\hline Cell culture plate (96-, 12- and 6-well) & Greiner bio-one, Kremsmuenster, Austria \\
\hline Cell culture flask $\left(75 \mathrm{~cm}^{2}\right.$ and $\left.175 \mathrm{~cm}^{2}\right)$ & Greiner bio-one, Kremsmuenster, Austria \\
\hline Cell scraper & Sarstedt, Nürnbrecht, Germany \\
\hline C-Tubes & Miltenyi Biotec, Bergisch Gladbach, Germany \\
\hline FACS tubes & Corning, Corning, NY, USA \\
\hline Falcon $40 \mu \mathrm{M}$ cell strainer & Corning, Corning, NY, USA \\
\hline Insulin syringes ( $0.5 \mathrm{~mL} / 50$ I.U.) & B.Braun, Melsungen, Germany \\
\hline $\begin{array}{l}\text { Microtest plate, flat bottom and u-bottom shape } \\
\text { (96-well) }\end{array}$ & Sarstedt, Nürnbrecht, Germany \\
\hline Needles & B.Braun, Melsungen, Germany \\
\hline Nunc MaxiSorp ${ }^{\circledR}$ plates & Thermo Fisher Scientific, Waltham, MA, USA \\
\hline PCR 8-tube strips & $\begin{array}{l}\text { Biozym Scientific GmbH, Hessisch Oldendorf, } \\
\text { Germany }\end{array}$ \\
\hline PCR plate (96-well) & Sarstedt, Nürnbrecht, Germany \\
\hline Petri dish $(3 \mathrm{~cm})$ & Greiner bio-one, Kremsmuenster, Austria \\
\hline Petri dish $(10 \mathrm{~cm})$ & Sarstedt, Nürnbrecht, Germany \\
\hline Serological pipettes $(5,10$ and $25 \mathrm{~mL})$ & Sarstedt, Nürnbrecht, Germany \\
\hline Syringes & B.Braun, Melsungen, Germany \\
\hline Tubes $(50,15,10,1.5,0.5$ and $0.2 \mathrm{~mL})$ & Sarstedt, Nürnbrecht, Germany \\
\hline
\end{tabular}

\subsubsection{Software and technical devices}

Table 3.13: Software

\begin{tabular}{|c|c|c|}
\hline Software & Application & Source of supply \\
\hline FlowJo v10 & Analysis of flow cytometry data & FlowJo, LLC, Ashland, OR, USA \\
\hline GraphPad Prism 6 & Statistical analysis and graphs & $\begin{array}{l}\text { GraphPad software Inc., La Jolla, CA, } \\
\text { USA }\end{array}$ \\
\hline $\begin{array}{l}\text { Quant Studio }{ }^{\text {TM }} \text { Real- } \\
\text { Time PCR Software }\end{array}$ & Analysis of real-time PCR data & $\begin{array}{l}\text { Thermo Fisher Scientific, Waltham, } \\
\text { MA, USA }\end{array}$ \\
\hline
\end{tabular}

$\mathrm{RT}^{2}$ Profiler PCR Ar- Analysis of $\mathrm{RT}^{2}$ Profiler $^{\mathrm{TM}}$ PCR Qiagen, Hilden, Germany

ray Data Analysis V4 array data 
Table 3.14: Technical devices

\section{Device}

BD LSR Fortessa ${ }^{\mathrm{TM}}$

\begin{tabular}{ll}
\hline Centrifuge 5418 R & Eppendorf, Hamburg, Germany \\
\hline Centrifuge 5810 R & Eppendorf, Hamburg, Germany \\
\hline gentleMACS $^{\mathrm{TM}}$ Dissociator & Miltenyi Biotec, Bergisch Gladbach, Germany \\
\hline iMark $^{\mathrm{TM}}$ Microplate Absorbance Reader & Bio-Rad, Hercules, CA, USA \\
\hline Incubator CellStar $^{\text {Nunc/Thermo Fisher Scientific, Waltham, MA, }}$ \\
\hline
\end{tabular}

Olympus, Hamburg, Germany

Leica, Wetzlar, Germany

Peqlab, VWR International GmbH, Erlangen, Germany

\begin{tabular}{ll}
\hline Quant Studio $^{\text {TM }} 7$ Flex Real-Time PCR System & Thermo Fisher Scientific, Waltham, MA, USA \\
\hline Sonicator UW 2070 & Bandelin electronic, Berlin, Germany \\
\hline Stereotactic device & Stoelting Co, Wood Dale, IL, USA \\
\hline Tissue Processor TP 1020 & Leica, Wetzlar, Germany
\end{tabular}

\subsection{Animals}

C57BL/6 wildtype mice (wt) and IFNAR1- (ifnar $\left.1^{-\%}\right)$, Tyk2- (tyk2 $\left.{ }^{E 775 K}\right)$, STAT1- (stat1 $\left.{ }^{-/}\right)$, STAT2- (B6.129-

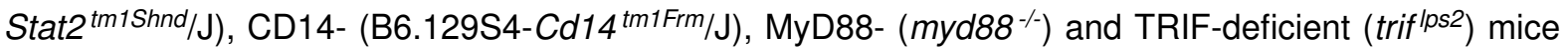
were bred and obtained from the central animal facility of the University Medicine Göttingen and housed under standard or specific pathogen-free (SPF) conditions. Tyk2 ${ }^{E 775 K}$, stat2 ${ }^{\%}$ and $c d 14^{-/}$mice were from the Jackson Laboratory. Ifnar $1 \%$ mice were kindly provided by Prof. Dr. Marco Prinz, Freiburg, Germany, stat ${ }^{\%}$ mice by Prof. Dr. Thomas Meyer, Göttingen, Germany, myd88\% mice by Prof. Dr. Shizuo Akira, Osaka, Japan and trif ${ }^{1 / s 2}$ mice by Prof. Dr. Bruce Beutler, La Jolla, USA. All animals were treated according to the guidelines for animal care of the University Medicine Göttingen.

\subsection{Cell culture}

\subsubsection{Primary mouse microglial cultures}

For the preparation of primary microglial cell cultures, whole brains of neonatal (P0) mice were excised. The meninges were removed and the brains washed three times with HBSS. Afterwards, cells were incubated for $5 \mathrm{~min}$ at $37^{\circ} \mathrm{C}$ with $2.5 \%$ trypsin, vortexed gently and incubated for another $5 \mathrm{~min}$ at $37^{\circ} \mathrm{C}$. The digestion was stopped with complete culture medium. Next, the cell suspension was incubated with $0.4 \mathrm{mg} / \mathrm{mL}$ DNase for $2-3 \mathrm{~min}$ at $37^{\circ} \mathrm{C}$. Remaining cell clumps were removed by careful resuspension and cells were centrifuged at $200 \mathrm{xg}$ for $10 \mathrm{~min}$ at $4^{\circ} \mathrm{C}$. After centrifugation, the supernatant was removed and cells were resuspended in fresh complete culture medium. Cells were seeded into $75 \mathrm{~cm}^{2}$ 
culture flasks that were coated beforehand with $100 \mu \mathrm{g} / \mathrm{mL}$ poly-L-lysine (PLL) for at least $30 \mathrm{~min}$ followed by three times washing with aqua ${ }_{b i d e s t}$ and once with culture medium. Cultures were kept in an incubator adjusted to $37^{\circ} \mathrm{C}$ and $5 \% \mathrm{CO}_{2}$.

The next day, cultures were washed three times with PBS, once with complete culture medium and afterwards, fresh culture medium was added. On the following day, the culture medium was changed again. One week later, cells received complete culture medium containing 30\% L929-conditioned cell culture supernatant to stimulate microglial proliferation. Five days later microglia were harvested. For this purpose microglia were shaked off the astrocytic feeder layer for at least $30 \mathrm{~min}$ at $37^{\circ} \mathrm{C}$ with a speed of $140 \mathrm{rpm}$. The supernatant containing microglia was collected, cells were washed and the cell number was determined. Microglia were plated in complete culture medium in 96-well plates with 15,000 cells/well, in 12-well plates with 200,000 cells/well and in 6 -well plates with 800,000 cells/well.

The remaining cells were re-stimulated with complete culture medium containing $30 \%$ L929-conditioned cell culture supernatant. Using this procedure, cells can be harvested again on day 17, 22 and 24 .

\subsubsection{Bone marrow-derived macrophage cultures}

Bone marrow-derived macrophages were isolated from 8-12 week old mice. Therefore, mice were sacrificed by cervical dislocation and femurs were isolated by using the scissors and forceps. The bones were cleaned from the muscle tissue and cut on both ends near the joints. Bone marrow was washed out of the femur by using a $5 \mathrm{~mL}$ syringe with a small needle $(0.45 \mathrm{~mm}$ diameter) filled with Pluznik medium. The bone marrow from two femurs was placed in a $10 \mathrm{~cm}$ Petri dish containing $10 \mathrm{~mL}$ Pluznik medium and kept in an incubator adjusted to $37^{\circ} \mathrm{C}$ and $5 \% \mathrm{CO}_{2}$ overnight. The next day, the medium containing the cells was collected into a $50 \mathrm{~mL}$ Falcon tube and centrifuged at $300 \mathrm{xg}$ for $10 \mathrm{~min}$ at $4{ }^{\circ} \mathrm{C}$. The supernatant was discarded, cells were resuspended in $40 \mathrm{~mL}$ Pluznik medium and cultured in four new Petri dishes. Medium was changed on day 4 and BMDM were harvested on day 7 by incubation with $5 \mathrm{~mL} 4 \mathrm{mM}$ EDTA for $15 \mathrm{~min}$ at $37^{\circ} \mathrm{C}$. Cells were washed, counted and plated in complete culture medium in 96 -well plates with 15,000 cells/well.

\subsubsection{Peritoneal macrophage cultures}

Peritoneal macrophages were isolated from 8-12 week old mice. Therefore, mice were sacrificed by cervical dislocation and the peritoneum was exposed by carefully removing the skin above. Through a small cut in the peritoneum, $1 \mathrm{~mL}$ ice-cold PBS was injected into the peritoneal cavitiy by using a $1000 \mu \mathrm{L}$ pipette. The abdomen was gently massaged to loosen the macrophages and the fluid was collected into a pre-cooled $15 \mathrm{~mL}$ Falcon tube. This procedure was repeated six to eight times. Afterwards, cells were washed, counted and plated in complete culture medium in 96-well plates with 25,000 cells/well.

\subsubsection{L929 fibroblast cultures}

L929 fibroblasts were cultured in order to obtain L929-conditioned cell culture supernatant for stimulation of microglial proliferation. Therefore, L929 fibroblasts were cultured in two $75 \mathrm{~cm}^{2}$ culture flasks in complete culture medium. After one week, cells were collected and split into two $75 \mathrm{~cm}^{2}$ culture flasks for further cultivation and three $175 \mathrm{~cm}^{2}$ culture flasks that were cultivated for two weeks without medium change. Supernatants were collected from the $175 \mathrm{~cm}^{2}$ culture flasks and stored at $-20^{\circ} \mathrm{C}$ until used for microglial cultures. L929 fibroblasts were cultured for 30 passages and afterwards replaced by fresh cultures established from a liquid nitrogen stock. 
L929 fibroblasts were also cultured in order to obtain L929-conditioned medium for stimulation of BMDM proliferation. Therefore, L929 fibroblasts were seeded at a density of $1 \times 10^{7}$ cells in $100 \mathrm{~mL}$ complete culture medium. After cultivation for one week without medium change, the medium was collected, sterile filtered and stored at $-20^{\circ} \mathrm{C}$ until used for BMDM cultures.

\subsection{In vitro stimulation of cells and determination of cell viability}

Microglia, BMDM and peritoneal macrophages from different mouse strains were stimulated with several TLR agonists or interferons (see Table 3.1). All stimulating agents were diluted to the respective working concentration in complete culture medium prior to use. The medium, in which the cells were plated before, was exchanged for the medium containing the stimulating agent. Cells were then incubated for a certain period of time at $37^{\circ} \mathrm{C}$ and $5 \% \mathrm{CO}_{2}$ depending on the experimental setup. If inhibitors (see Table 3.3) or blocking antibodies (see Table 3.6) were used, cells were pre-incubated for $1 \mathrm{~h}$ with the respective compound before stimulation.

The cell viability was determined via a WST-1 assay to rule out toxicity of the different stimulating agents, inhibitors and blocking antibodies. For this purpose cells received fresh complete culture medium supplemented with $10 \%$ WST-1 after removal of the different compounds and were incubated for $3 \mathrm{~h}$ at $37^{\circ} \mathrm{C}$. WST-1 is a tetrazolium salt that is cleaved by the succinyltetrazolium reductase, which is part of the mitochondrial respiratory chain and therefore indicates metabolic activity of cells. The cleavage results in formation of the soluble dye formazan. This color reaction was measured in a microplate reader at $450 \mathrm{~nm}$ with $655 \mathrm{~nm}$ as reference wavelength.

\subsection{Quantification of chemokines and interferons by ELISA}

The release of chemokines and interferons was quantified with enzyme-linked immunoabsorbent assay (ELISA) using different kits. Levels of CXCL1, CCL2, IFN $\alpha$ and IFN $\beta$ were determined in cell culture supernatants or brain lysates. Assays were performed on Nunc MaxiSorp ${ }^{\circledR}$ plates according to the manufacturer's instructions. Absorbance was measured in a microplate reader at $450 \mathrm{~nm}$ with $540 \mathrm{~nm}$ as reference wavelength.

Cell culture supernatants for measuring the production of chemokines and interferons by cultured microglia (15,000/well in 96-well plate), BMDM (15,000/well in 96-well plate) and peritoneal macrophages $(25,000 /$ well in 96-well plate) were obtained from in vitro stimulation experiments after $18 \mathrm{~h}$, unless stated otherwise, and stored at $-20^{\circ} \mathrm{C}$ until assayed.

Brain lysates for measuring intracerebral chemokine production were prepared as follows. One half of an adult mouse brain was lysed by adding $500 \mu \mathrm{L}$ lysis buffer containing $2 \mathrm{nM}$ PMSF for $5 \mathrm{~min}$ of ice. Afterwards, cells were disrupted by sonication. Cell debris was removed by centrifugation at $14,000 \times \mathrm{g}$ for $10 \mathrm{~min}$ at $4{ }^{\circ} \mathrm{C}$. The supernatant was collected and stored at $-20^{\circ} \mathrm{C}$ until assayed.

\subsection{Real-time PCR and $\mathrm{RT}^{2}$ Profiler $^{\mathrm{TM}}$ PCR array}

Total RNA was isolated from cultured microglia (800,000/well in 6-well plate) $3 \mathrm{~h}$ after in vitro stimulation by using the RNeasy Mini Kit according to the manufacturer's instructions.

For real-time PCR (RT-PCR), 300 ng of purified RNA were transcribed into cDNA by using the Quanti Tect ${ }^{\circledR}$ Reverse Transcription Kit according to the manufacturer's instructions. RT-PCR was performed by using the $\mathrm{iTaq}^{\mathrm{TM}}$ Universal SYBR ${ }^{\circledR}$ Green Supermix according to the manufacturer's instructions. 
PrimePCR ${ }^{\mathrm{TM}}$ SYBR $^{\circledR}$ Green Assay primer for ifnab, ifnb1, stat1, stat2 and stat3 were used. The housekeeping gene gapdh served as internal control.

For $\mathrm{RT}^{2}$ Profiler ${ }^{\mathrm{TM}} \mathrm{PCR}$ array, $500 \mathrm{ng}$ of purified RNA were transcribed into cDNA by using the $\mathrm{RT}^{2}$ First Strand Kit according to the manufacturer's instructions. CDNA was mixed with the $\mathrm{RT}^{2}$ Profiler PCR Array PCR components and dispensed into the array plate for performing real-time PCR according to the manufacturer's instructions. Data was analyzed with the web-based PCR Array Data Analysis Software provided by the manufacturer.

\subsection{Flow cytometry analysis of E.coli and myelin phagocytosis}

Microglial phagocytosis of bacteria was assessed by using Discosoma red fluorescent protein (DsRed)expressing DH5 $\alpha$ E.coli. Cultured microglia (200,000/well in 12-well plate) were exposed to the bacterial solution containing $2 \times 10^{6}$ DsRed DH5 $\alpha$ E.coli in culture medium without penicillin / streptomycin, but with $100 \mu \mathrm{g} / \mathrm{mL}$ ampicillin. After $2 \mathrm{~h}$ incubation at $37^{\circ} \mathrm{C}$ and $5 \% \mathrm{CO}_{2}$, the bacterial solution was removed and remaining bacteria were killed by incubation with $100 \mu \mathrm{g} / \mathrm{mL}$ gentamicin for $1 \mathrm{~h}$ at $37^{\circ} \mathrm{C}$ and $5 \%$ $\mathrm{CO}_{2}$. Subsequently, cells were processed for flow cytometry analysis.

Microglial phagocytosis of myelin was assessed by using myelin, which was labeled with the DyLight ${ }^{\mathrm{TM}}$ 550 Labeling Kit according to the manufacturer's instructions. Cultured microglia (200,000/well in 12well plate) were incubated in the presence of DyLight 550 myelin for $2 \mathrm{~h}$ at $37^{\circ} \mathrm{C}$ and $5 \% \mathrm{CO}_{2}$, the myelin solution was removed and cells were processed for flow cytometry analysis.

For that purpose, cells were washed once with culture medium and twice with PBS and detached from the plate by incubation with $0.05 \% / 0.02 \%$ trypsin $/$ EDTA for $3-5 \mathrm{~min}$ at $37^{\circ} \mathrm{C}$. The enzymatic reaction was stopped by adding fresh complete culture medium. Afterwards, cells were scraped carefully and transferred into $2 \mathrm{~mL}$ Eppendorf cups for subsequent centrifugation at $800 \times \mathrm{g}$ for $10 \mathrm{~min}$ at $4^{\circ} \mathrm{C}$. After washing with FACS buffer, Fc receptors were blocked with an anti-mouse CD16/CD32 antibody for 10 min at $4{ }^{\circ} \mathrm{C}$. Finally, cells were stained with an APC anti-mouse CD11b antibody for 25 min at $4{ }^{\circ} \mathrm{C}$ in the dark and washed once again. Samples were acquired on BD LSR Fortessa ${ }^{\text {TM }}$ and 10,000 CD11 $\mathrm{b}^{+}$ cells were included for data analysis. Data was evaluated with the software FlowJo.

\subsection{Intracerebral E. coli and S. pneumoniae infection}

Intracerebral injections of E. coli K1 and S. pneumoniae D39 were used as a model of gram-negative and gram-positive meningitis, respectively. E. coli $\mathrm{K} 1$ is an encapsulated, pathogenic strain that was originally isolated from the cerebrospinal fluid of a child with neonatal meningitis (Ribes et al. 2013). It was provided by Dr. G. Zysk, Institute for Medical Microbiology and Virology, Düsseldorf, Germany. S. pneumoniae D39 is an encapsulated strain that was isolated from a patient in 1916 (Lanie et al. 2007). It was provided by Prof. Dr. S. Hammerschmidt, Department of Genetics of Microorganisms, Greifswald, Germany. Both strains were passaged through mice, grown overnight on blood agar plates, harvested with $0.9 \%$ saline and stored at $-80^{\circ} \mathrm{C}$. Frozen aliquots (generously provided by Dr. Sandra Ribes, Institute for Neuropathology, Göttingen, Germany) were used for the experiments.

In order to induce meningitis, 8-12 week old mice were anesthesized with ketamine $(60 \mathrm{mg} / \mathrm{kg}$ bodyweight) and xylazine ( $8 \mathrm{mg} / \mathrm{kg}$ bodyweight). Bacteria were diluted in saline and injected into the right frontal neocortex with a concentration of 2000-5000 CFU/mouse for E. coli and 500-800 CFU/mouse for S. pneumoniae. As a control, mice were either injected with PBS or not injected. After $24 \mathrm{~h}$, mice were sacrificed by i.p. injection of $14 \%$ chloralhydrate. Blood was taken directly from the heart, plated on blood agar plates and incubated for $24 \mathrm{~h}$ at $37^{\circ} \mathrm{C}$ in order to quantify the severeness of the infection 
by determining the number of bacteria in the blood. Mice were perfused with PBS via the left ventricle of the heart, brains were dissected and further processed as described in chapter 3.10.

\subsection{Induction of focal NMO-like lesions in mice}

Focal NMO-like lesions were induced in 8-12 week old mice by intracortical injection of a human recombinant anti-AQP4 antibody (referred to as NMO-Ab) together with human complement. Therefore, mice were anesthesized as described in chapter 3.8, their skull was shaved and they were placed in a stereotactic frame. A rostro-caudal cut was performed to expose the skull and a small hole was drilled $0.1 \mathrm{~mm}$ caudal and $0.2 \mathrm{~mm}$ lateral to the bregma. The last thin layer of the bone was carefully cut with a microdissecting knife to avoid damaging the brain. $1 \mu \mathrm{L}$ antibody mixture containing $15 \mathrm{U} / \mathrm{mL}$ human complement, $2.5 \mathrm{mg} / \mathrm{mL}$ NMO-Ab and the tracer dye Monastral Blue was slowly injected into the cortex with a fine glass capillary. As a control, mice were either injected with a CNS unspecific human recombinant antibody directed against measles virus nucleocapsid protein (referred to as CTL-Ab) together with human complement or injected with PBS. All animals were injected into both hemispheres. Subsequently, the capillary was slowly withdrawn and the skin was closed by a suture. After $24 \mathrm{~h}$, mice were sacrificed by i.p. injection of $14 \%$ chloralhydrate and perfused with PBS via the left ventricle of the heart. The brains were dissected and halved. One half was processed as described in chapter 3.10 for flow cytometry analysis and one half was transferred into 4\% PFA and processed as described in chapter 3.11 for histological analysis.

\subsection{Preparation of single-cell suspensions from brain tissue for flow cytometry analysis}

Single-cell suspensions for flow cytometry analysis were prepared from perfused brains using the Neural Tissue Dissociation Kit (T). The brain tissue was dissociated following the manufacturer's instructions for automated dissociation using the gentleMACS ${ }^{\mathrm{TM}}$ Dissociator. Afterwards, myelin was removed by a Percoll gradient. Therefore, the cell suspension was resuspended in $5 \mathrm{~mL} 37 \%$ Percoll in complete culture medium and layered over $4 \mathrm{~mL} 70 \%$ Percoll in complete culture medium in a $15 \mathrm{~mL}$ Falcon tube. After centrifugation at $500 \mathrm{xg}$ for $25 \mathrm{~min}$ at $4^{\circ} \mathrm{C}$ without acceleration and brakes, the myelin debris was removed and the cells were collected at the interphase. The cells were transferred to FACS tubes and washed with FACS buffer. Fc receptors were blocked with an anti-mouse CD16/CD32 antibody for $10 \mathrm{~min}$ at $4^{\circ} \mathrm{C}$ and cells were stained for $25 \mathrm{~min}$ at $4{ }^{\circ} \mathrm{C}$ in the dark with the following antibodies: FITC anti-mouse CD11b, PerCP-Cy5.5 anti-mouse CD45, Pacific Blue anti-mouse Ly6G, APC anti-mouse Ly6C and PE anti-mouse CD3. After washing, samples were acquired on BD LSR Fortessa ${ }^{\mathrm{TM}}$ and $10,000 \mathrm{CD} 11 \mathrm{~b}^{+}$cells were included for data analysis. Data was evaluated with the software FlowJo.

\subsection{Histology of focal NMO-like lesions}

\subsubsection{Preparation for histology}

For histological analysis, perfused brains were fixed for at least 2 days at $4^{\circ} \mathrm{C}$ in Falcon tubes containing 4\% PFA solution and afterwards washed with PBS. The tissue was dehydrated via alcohol / xylene and embedded in paraffin blocks using the Leica TP 1020 Tissue Processor according to the standard 
procedure. The paraffin blocks were cut with a sliding microtome into $2-3 \mu \mathrm{m}$ thin coronal sections and transferred to object slides.

Prior to the staining procedure, the tissue was deparaffinized for at least $1 \mathrm{~h}$ at $56^{\circ} \mathrm{C}$. Subsequent rehydration was performed using the following xylene / alcohol solutions for the indicated time period: xylene ( $4 \times 5 \mathrm{~min})$, isoxylene ( $1 \times 2 \mathrm{~min}), 100 \%$ isopropyl alcohol $(2 \times 3 \mathrm{~min}), 90 \%$ isopropyl alcohol ( $1 \times 3 \mathrm{~min}) 70 \%$ isopropyl alcohol $(1 \times 3 \mathrm{~min})$ and $50 \%$ isopropyl alcohol $(1 \times 3 \mathrm{~min})$. In the end, sections were washed with aqua bidest $_{\text {. }}$.

After the staining procedure, sections were dehydrated by performing the above described rehydration steps in reverse order and mounted in DePeX medium.

\subsubsection{Hematoxylin and eosin (HE) staining}

Hematoxylin and eosin (HE) staining was used for gaining a general overview of the tissue morphology and inflammation. Therefore, deparaffinized sections were incubated for $5 \mathrm{~min}$ with Mayer's hemalum solution, washed with aqua ${ }_{\text {bidest }}$ and shortly differentiated in $1 \% \mathrm{HCl}$-alcohol. After blueing in water for $10 \mathrm{~min}$, sections were incubated with $1 \%$ eosin solution for $5 \mathrm{~min}$ and subsequently washed with aqua $_{\text {bidest }}$. Finally, sections were dehydrated and mounted.

\subsubsection{Aquaporin 4 (AQP4) staining}

Aquaporin 4 (AQP4) staining was performed for analysis of astrocyte pathology and lesion formation. Therefore, deparaffinized sections were washed with PBS and endogenous peroxidases were blocked by incubation for $10 \mathrm{~min}$ with $3 \%$ hydrogen peroxide solution. Sections were washed three times with PBS and blocked by incubation for $10 \mathrm{~min}$ with blocking buffer. The primary antibody directed against AQP4, diluted in blocking buffer, was added and sections were incubated over night at $4^{\circ} \mathrm{C}$. The next day, sections were washed three times with PBS and incubated for $1 \mathrm{~h}$ at RT with the secondary antibody, diluted in blocking buffer. After three times washing with PBS, $0.1 \%$ streptavidin horseradish peroxidase in blocking buffer was added and sections were incubated for $45 \mathrm{~min}$ at RT. Following three times washing with PBS, the staining was developed with a 3,3' diaminobenzidine (DAB) solution and the staining intensity was controlled under a microscope. Sections were washed with aqua $a_{b i d e s t}$ and counterstained in Mayer's hemalum solution for $0.5 \mathrm{~min}$. Afterwards, sections were washed with aqua $\mathrm{a}_{\text {bidest }}$ and shortly differentiated in $1 \% \mathrm{HCl}$-alcohol. Finally, sections were blued in water for $7 \mathrm{~min}$, dehydrated and mounted.

\subsubsection{Chloroacetate esterase (CAE) enzyme histochemistry and acquisition of neutrophil numbers}

Chloroacetate esterase (CAE) staining was used in order to specifically stain neutrophils in the brain tissue. Therefore, deparaffinized sections were pre-warmed in PBS at $37^{\circ} \mathrm{C}$ for $1 \mathrm{~h}$, stained with the naphthol AS-D chloroacetate (specific esterase) kit according to the manufacturer's instructions and then mounted using Aquamount.

In order to determine the number of neutrophils, $\mathrm{CAE}^{+}$cells were counted in the stained sections. Therefore, a light microscope with an ocular morphometric grid was used and all cells in the area around the injection site were counted at a 20x magnification. 


\subsection{Statistical analysis}

Statistical analysis was performed using the GraphPad Prism 6 software. The Mann-Whitney test was used for non-parametric data to compare two independent groups. For the comparison of more than two groups, the two-way ANOVA with either Sidak's correction or Turkey's correction was used. Statistical significance was defined as $p<0.05$ and marked as follows: ${ }^{*} p \leq 0.05,{ }^{* *} p \leq 0.01,{ }^{* * *} p \leq 0.001$, ${ }^{* * * *} p \leq 0.0001$. All data are mean \pm SEM. 


\section{Results}

\subsection{Microglial production of type I interferons}

Interferons are important immune mediators that play a crucial role in fighting viral, but also bacterial infections. They can be produced upon activation of TLRs, which recognize several PAMPs that indicate the presence of pathogens (Trinchieri 2010). Within the CNS, microglia as the principal immune cells serve important functions in eradicating invading pathogens. They are equipped with a large variety of TLRs and potently produce several inflammatory and immunoregulatory factors, such as cytokines and chemokines, upon activation of these receptors (Kettenmann et al. 2011). Thus, microglia could shape immune responses within the CNS by producing different interferons in response to various pathogens.

\subsubsection{Microglia produce IFN $\beta$, but hardly any IFN $\alpha$ upon TLR stimulation}

Type I interferons are the largest subgroup of interferons. This family consists of 8 different interferon species of which IFN $\alpha$ and IFN $\beta$ are the most important ones (Pestka 2007). They can be produced by many different cell types in response to a variety of stimuli. Therefore, the capability of microglia to produce IFN $\alpha$ and IFN $\beta$ upon activation of different TLRs was assessed.

Cultured, primary microglia were stimulated in vitro with Re-LPS, S-LPS, live E. coli or FN as agonists for TLR4, Pam3CSK4 as agonist for TLR1/2, MALP-2 as agonist for TLR6/2 or poly I:C as agonist for TLR3. After $18 \mathrm{~h}$, supernatants containing soluble factors were collected and IFN $\beta$ release was determined by ELISA. While TLR2 agonists completely failed to induce IFN $\beta$ production, it was massively released after poly I:C treatment (Figure 4.1 A). An intermediate production of about $0.1-0.15 \mathrm{ng} / \mathrm{mL}$ was triggered by all different TLR4 agonists. Surprisingly, not only the bacterial ligands, but also the DAMP FN similarly led to IFN $\beta$ production, indicating a role of interferons also in tissue damage. This intermediate production could be further increased depending on the dose of the stimulus. Re- or S-LPS were slightly more potent stimulators of IFN $\beta$ than FN inducing a maximal release of about $0.3-0.4 \mathrm{ng} / \mathrm{mL}$, while $\mathrm{FN}$ triggered $0.25-0.3 \mathrm{ng} / \mathrm{mL}$ (Figure $4.1 \mathrm{C}$ and $\mathrm{D}$ ). This maximal release was obtained rather quickly. A time profile of Re-LPS-triggered IFN $\beta$ release revealed that a plateau is reached already after $6 \mathrm{~h}$, which stays stable even over $48 \mathrm{~h}$ (Figure $4.1 \mathrm{~B}$ ). Thus, IFN $\beta$ seems to belong to the early cytokines that are produced after pathogen encounter.

Furthermore, the release of IFN $\alpha$ was analyzed $18 \mathrm{~h}$ after stimulation with Re-LPS, Pam3CSK4, MALP2 and poly I:C. ELISA measurements showed that only TLR3 activation leads to IFN $\alpha$ production, while neither TLR4 nor TLR2 agonists had any effect (Figure 4.2 A). To rule out that sensitivity problems of the ELISA kit caused false negative results, mRNA expression of IFN $\alpha$ was determined. A RT-PCR array including seven different IFN $\alpha$ subtypes as well as $\operatorname{IFN} \beta, \operatorname{IFN} \epsilon, \operatorname{IFN} \kappa$ and IFN $\zeta$ was performed in order to get a general overview about regulation of type I interferon expression upon TLR challenges. Re-LPS and Pam3CSK4 served as respective examples for the TLR challenge. First, the Re-LPS- and Pam3CSK4-triggered relative gene expression, calculated by $2^{-\Delta c_{T}}$, of all 89 genes included in the array 
A

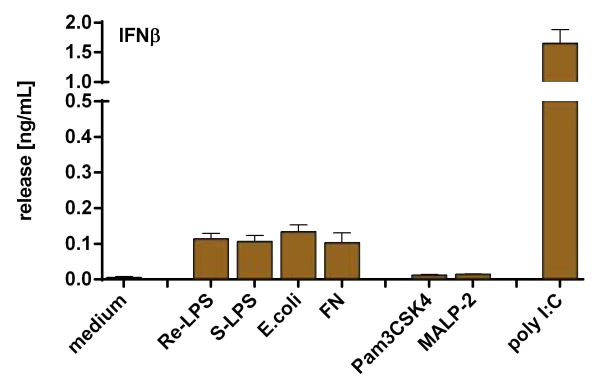

C

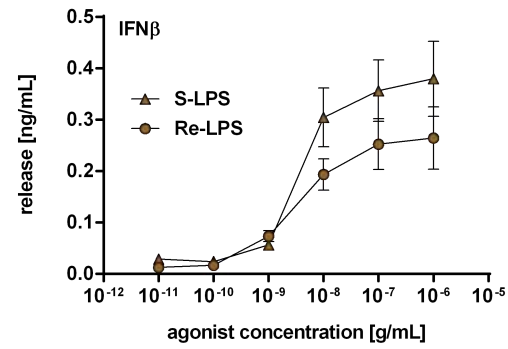

B

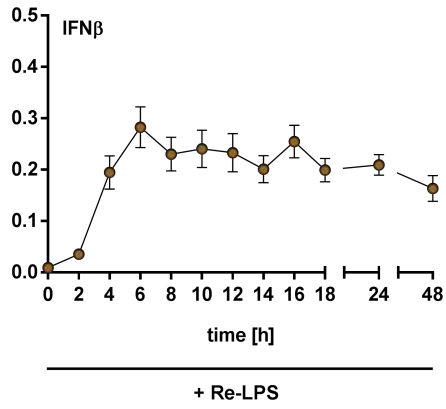

D

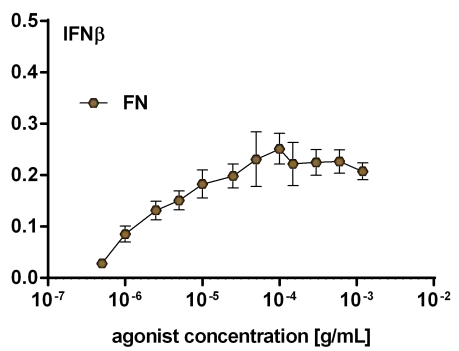

Figure 4.1: Microglia produce IFN $\beta$ upon stimulation with several TLR agonists. Cultured microglia from wt mice were stimulated with (A) $10 \mathrm{ng} / \mathrm{mL}$ Re-LPS, S-LPS, Pam3CSK4, MALP-2, $2 \times 10^{5} \mathrm{CFU} / \mathrm{mL}$ E. coli DsRed DH5 $\alpha, 100 \mu \mathrm{g} / \mathrm{mL}$ FN or $50 \mu \mathrm{g} / \mathrm{mL}$ poly I:C for $18 \mathrm{~h}$ or (B) $10 \mathrm{ng} / \mathrm{mL}$ Re-LPS for the indicated time points or increasing doses of (C) Re-LPS, S-LPS or (D) FN as indicated for $18 \mathrm{~h}$. IFN $\beta$ release was determined in cell culture supernatants by ELISA. Data are mean $\pm \mathrm{SEM}$ with $\mathrm{n}=12$ from 3 independent experiments. Data from (B) and (C) is already published in Janova et al. (2016).

was correlated (Figure 4.2B). The middle line indicates perfect correlation and both other lines mark the confidence interval. Many genes were higher expressed upon TLR4 than TLR1/2 stimulation as shown by all dots below the lines. Only one gene was slightly more expressed in TLR1/2-stimulated microglia, namely IFN $\alpha$ B. For a more detailed analysis, the fold regulation of only type I interferons is shown in Figure 4.2 C. The fold regulation thereby represents the quotient of the relative gene expression of the Re-LPS- and the Pam3CSK4-stimulated sample. Values greater than 1 indicate an up-regulation, while values less than -1 indicate a down-regulation. The expression of most of the interferons was not different between both conditions. IFN $\alpha$ B appeared to be the only gene that was slightly more expressed in TLR1/2- than TLR4-stimulated microglia as it was already shown by the correlation analysis. Furthermore, in line with the previously described ELISA data, IFN $\beta$ was almost exclusively expressed in TLR4-stimulated cells. Because the RT-PCR array could be performed only once, the obtained results should be confirmed by RT-PCR analysis. In this case, cells were stimulated with ReLPS, FN, Pam3CSK4, MALP-2 or poly I:C for $3 \mathrm{~h}$ and afterwards the relative gene expression of IFN $\beta$ and IFN $\alpha$ was determined. Since there exist 14 IFN $\alpha$ subtypes in mice (van Pesch et al. 2004) and not all can be covered by using one primer pair, the expression of IFN $\alpha B, 6,12$ and 14 was characterized, which included the one subtype that showed off in the RT-PCR array. According to the ELISA data, only TLR4 and TLR3 agonists induced IFN $\beta$ gene expression (Figure 4.2D). Interestingly, the mRNA expression did not correlate with the amount of released protein. FN-induced expression levels were four times higher than Re-LPS-induced expression levels, even though both ligands led to nearly the same IFN $\beta$ release. Furthermore, poly I:C induced a rather low relative gene expression compared to the massively triggered protein production. Surprisingly, in neither of the conditions IFN $\alpha B, 6,12$ or 14 expression could be observed. Even poly I:C stimulation did not increase mRNA expression, indicating that probably another IFN $\alpha$ subtype was detected by the ELISA. Furthermore, this points towards a false positive result regarding IFN $\alpha$ B in the RT-PCR array. 
A

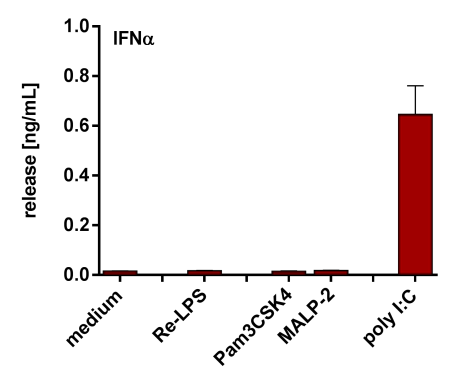

C

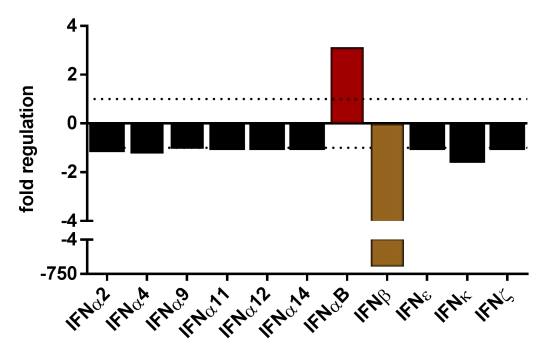

B

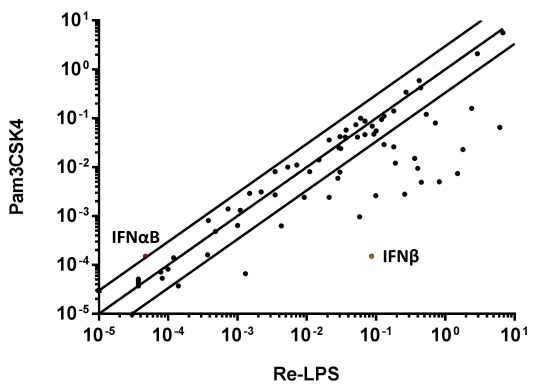

D

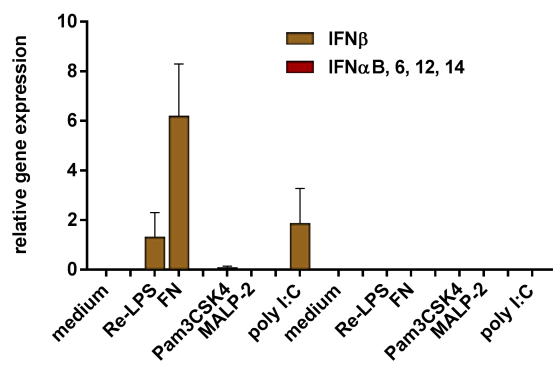

Figure 4.2: Microglia produce IFN $\alpha$ only in response to TLR3 stimulation. (A) Cultured microglia from wt mice were stimulated with $10 \mathrm{ng} / \mathrm{mL}$ Re-LPS, Pam3CSK4, MALP-2 or $50 \mu \mathrm{g} / \mathrm{mL}$ poly I:C for $18 \mathrm{~h}$. IFN $\alpha$ release was determined in cell culture supernatants by ELISA. Data are mean \pm SEM with $n=4-6$ from 2 independent experiments. (B), (C) Cultured microglia from wt mice were stimulated with $10 \mathrm{ng} / \mathrm{mL}$ Re-LPS or Pam3CSK4 for $3 \mathrm{~h}$. RNA was isolated by using the RNeasy Mini Kit and converted into cDNA by using the $\mathrm{RT}^{2}$ First Strand Kit. Real-Time PCR was performed by using a $\mathrm{RT}^{2}$ Profiler PCR Array. Relative gene expression was calculated by $2^{-\Delta C_{T}}$. Data are single values from 1 experiment. (B) Correlation analysis of Re-LPS- and Pam3CSK4-triggered relative gene expression of all 89 genes included in the $\mathrm{RT}^{2}$ Profiler PCR Array. The middle line indicates exact correlation, while both other lines represent the confidence interval. Dots above the upper line show genes that are more expressed in response to Pam3CSK4 than Re-LPS, while dots below the lower line indicate genes that are more expressed in response to Re-LPS than Pam3CSK4. IFN $\beta$ and IFN $\alpha$ B are marked in brown and red, respectively. (C) Comparison of the relative gene expression of different type I interferons in response to Re-LPS and Pam3CSK4. Fold regulation represents the quotient of Re-LPS- and Pam3CSK4-triggered relative gene expression. Values greater than 1 indicate an up-regulation, while values less than -1 indicate a down-regulation. IFN $\alpha \mathrm{B}$ and IFN $\beta$ are marked in red and brown, respectively. (D) Cultured microglia from wt mice were stimulated with $10 \mathrm{ng} / \mathrm{mL}$ Re-LPS, Pam3CSK4, MALP-2, $100 \mu \mathrm{g} / \mathrm{mL}$ FN or $50 \mu \mathrm{g} / \mathrm{mL}$ poly I:C for $3 \mathrm{~h}$. RNA was isolated by using the RNeasy Mini Kit and converted into cDNA by using the QuantiTect ${ }^{\circledR}$ Reverse Transcription Kit. Real-Time PCR was performed by using the iTaq ${ }^{\mathrm{TM}}$ Universal SYBR ${ }^{\circledR}$ Green Supermix. Relative gene expression was calculated by $2^{-\Delta \mathrm{C}_{\mathrm{T}}}$. Data are mean \pm SEM with $n=2-4$ from 2 independent experiments. IFN $\alpha$ B, 6, 12, 14 expression was partially measured and analyzed by Dr. Reza Khorooshi at the lab of Prof. Dr. Trevor Owens.

Overall, IFN $\beta$ was produced in this cell culture system after TLR challenges. Bacterial and damagerelated TLR4 and viral TLR3 ligands, but not the bacterial TLR2 agonists triggered IFN $\beta$ release by microglia. In contrast to that, the production of IFN $\alpha$ was exclusively linked to viral dsRNA as certain subtypes were only induced in response to TLR3 stimulation.

\subsubsection{IFN $\beta$ production is TRIF-dependent}

MyD88 and TRIF are the general adaptor proteins that initiate the TLR signaling pathway. The TRIFdependent activation of IRF3 is thereby the principal way to induce IFN $\beta$ (Fitzgerald et al. 2003). However, MyD88-dependent activation of IRF7 can under certain circumstances also lead to IFN $\beta$ production (Dietrich et al. 2010).

Because of this dual use of the two adaptor proteins, the dependence of microglial IFN $\beta$ release on MyD88 and/or TRIF was analyzed. Therefore, $m y d 88^{-\%}$ and trif ${ }^{1 p s 2}$ microglia were stimulated with different TLR agonists, the IFN $\beta$ production was measured by ELISA and compared to the production of wt cells. As described in chapter 4.1.1, substantial amounts of IFN $\beta$ were only induced by the TLR4 agonists Re-LPS, S-LPS and FN as well as by the TLR3 agonist poly I:C. This production was not al- 
A

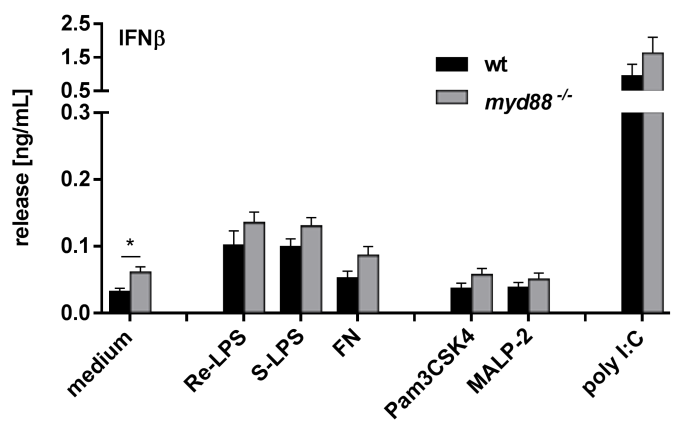

B

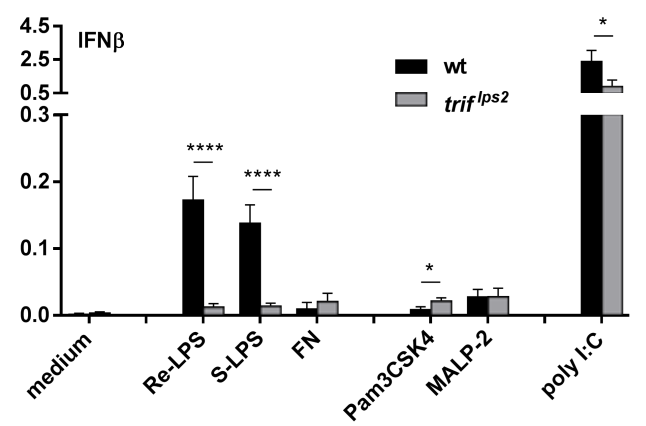

Figure 4.3: Microglial IFN $\beta$ production depends on TRIF, but not MyD88. Cultured microglia from wt and (A) $m y d 88^{-1}$ or (B) trif 1 ss 2 mice were stimulated with $10 \mathrm{ng} / \mathrm{mL}$ Re-LPS, S-LPS, Pam3CSK4, MALP-2, $100 \mu \mathrm{g} / \mathrm{mL}$ FN or $50 \mu \mathrm{g} / \mathrm{mL}$ poly I:C for $18 \mathrm{~h}$. IFN $\beta$ release was determined in cell culture supernatants by ELISA. Data are mean \pm SEM with $\mathrm{n}=12$ from 3 independent experiments. Statistical analysis was performed using the Mann-Whitney test, ${ }^{*}$ represents $p \leq 0.05$, ${ }^{* * * *}$ represents $p \leq 0.0001$.

tered by MyD88 deficiency (Figure 4.3 A) as wt and myd88 ${ }^{-/}$microglia produced the same amounts of IFN $\beta$. Surprisingly, basal IFN $\beta$ levels in unstimulated cells differed between both genotypes. In contrast to this, IFN $\beta$ levels massively depended on the presence of TRIF (Figure 4.3B). In the supernatants of Re- and S-LPS-stimulated trif/ps2 microglia, no IFN $\beta$ could be detected. Unfortunately, the effect of TRIF deficiency on FN-triggered IFN $\beta$ could not be analyzed as unexpectedly neither the wt nor the trif Ips2 microglia were able to react to this $\mathrm{FN}$ preparation. In response to poly I:C, the absence of TRIF decreased the IFN $\beta$ release by about $40 \%$. Thus, microglial IFN $\beta$ production in response to TLR 4 and TLR3 agonists depends on the functional presence of TRIF, but not MyD88.

\subsubsection{IFN $\beta$ production is absent in CD14-deficient microglia}

Within the TLR4 signaling cascade, IFN $\beta$ production is realized by the TRIF-dependent pathway as shown in chapter 4.1.2. This pathway is initiated from endosomes after internalization of the receptor complex. The endocytosis of TLR4 is a process that is controlled by CD14 (Zanoni et al. 2011). Accordingly, CD14 deficiency should affect IFN $\beta$ production similar to TRIF deficiency.

A

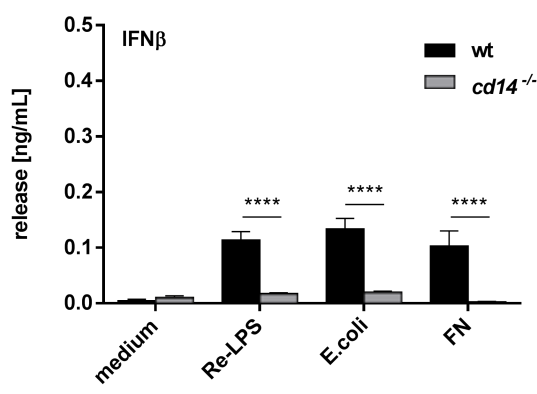

B

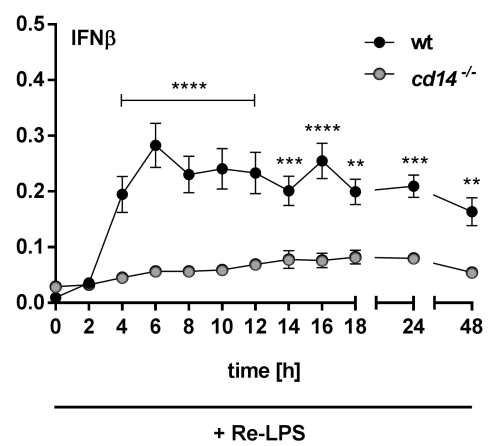

Figure 4.4: IFN $\beta$ production is completely absent in cd14 $\%$ microglia. Cultured microglia from wt and $c d 14^{-/}$mice were stimulated with (A) $10 \mathrm{ng} / \mathrm{mL}$ Re-LPS, $2 \times 10^{5} \mathrm{CFU} / \mathrm{mL}$ E. coli or $100 \mu \mathrm{g} / \mathrm{mL} \mathrm{FN}$ for $18 \mathrm{~h}$ or (B) $10 \mathrm{ng} / \mathrm{mL}$ Re-LPS for the indicated time points. IFN $\beta$ release was determined in cell culture supernatants by ELISA. Data are mean \pm SEM with $n=12$ from 3 independent experiments. (A) Statistical analysis was performed using the Mann-Whitney test or (B) compares both genotypes at each time point individually by using the two-way ANOVA with Sidak's correction, ${ }^{* *}$ represents $p \leq 0.01$, ${ }^{* * *}$ represents $p \leq 0.001$, ${ }^{* * * *}$ represents $\mathrm{p} \leq 0.0001$. Data from $(\boldsymbol{B})$ is already published in Janova et al. (2016). 
In order to test this hypothesis, $c d 14^{\%}$ and wt microglia were stimulated with Re-LPS, E.coli or FN and the IFN $\beta$ release was quantified after $18 \mathrm{~h}$. As expected, IFN $\beta$ production was completely absent in CD14-deficient cells, irrespective of the used TLR4 ligand (Figure 4.4A). To rule out that $c d 14^{-/}$ microglia are in principle able to release IFN $\beta$ in response to TLR 4 stimulation, but the production is just delayed, a time course study was performed. In this experiment, IFN $\beta$ levels upon stimulation with Re-LPS were measured in $c d 14^{-/}$and wt cells over $48 \mathrm{~h}$ with a $2 \mathrm{~h}$ analysis interval up to $18 \mathrm{~h}$. As shown in Figure 4.4B, no IFN $\beta$ was produced in the absence of CD14 throughout the whole time. Consequently, CD14 deficiency indeed leads to absent IFN $\beta$ release independent of the considered time frame. This indicates a direct link from CD14 to TRIF, which ultimately controls microglial IFN $\beta$ production in response to TLR4 challenges.

\subsection{Type I interferon signaling controls TLR4-induced chemokine production}

CD14 is a powerful regulator of microglial responses to TLR4 activation and shapes the outcome of infectious and non-infectious challenges (Regen et al. 2011; Janova et al. 2016). For reactions to DAMPs and tissue damage, the presence of CD14 is mandatory. Furthermore, it tightly controls the LPS-induced production of cytokines and chemokines. In the absence of CD14, microglial sensitivity to low amounts of LPS is dramatically reduced. Additionally, CD14 deficiency results in overreactions to massive LPS challenges. These are, amongst others, established in form of excessive CXCL1 production. In order to prevent such overreactions, microglia utilize an IFN $\beta$-mediated feedback mechanism. In this regard, CXCL1 production is negatively regulated by the presence of IFN $\beta$. As all other type I interferons, IFN $\beta$ signals via IFNAR and activates Jak1, Tyk2, STAT1 and STAT2 as the most important down-stream mediators. Since IFN $\beta$ prevents overshooting microglial reactions to strong infectious stimuli, the canonical type I interferon signaling pathway could be crucial for mediating regulatory effects on TLR4-triggered responses.

\subsubsection{Regulation of CXCL1 and CCL2 production depends on IFNAR1}

IFNAR is the type I interferon receptor and consists of two subunits, IFNAR1 and IFNAR2. Deficiency in one of the subunits disrupts the whole receptor and impairs ligand binding (Cohen et al. 1995). Hence, ifnar $1^{-}$mice served as model for receptor deficiency. In order to evaluate the contribution of canonical type I interferon signaling to the regulation of TLR4-mediated responses, ifnar ${ }^{\%}$ microglia were characterized in terms of their chemokine production. The measurements were focused on CXCL1 and CCL2, because CXCL1 is negatively regulated by IFN $\beta$, while CCL2 as a comparison is not (Janova et al. 2016). Thus, CXCL1 production could depend on IFNAR, whereas CCL2 would be probably independent.

According to that, wt and ifnar1 ${ }^{-}$microglia were stimulated with Re-LPS, S-LPS or FN to activate TLR4 and the release of CXCL1 and CCL2 was measured by ELISA. For all three agonists the presence of IFNAR1 was required for a normal CXCL1 production (Figure 4.5A). In the absence of IFNAR1, CXCL1 was up to twelvefold overproduced compared to the production by wt cells, indicating a dramatic dysregulation of this chemokine. In contrast to that, the CCL2 production in ifnar $1^{-1}$ microglia was even slightly reduced compared to wt (Figure 4.5B).

The observed results could be due to the IFNAR1 deficiency, but a decreased production of IFN $\beta$ and subsequent lack of negative regulation would have a similar effect. To exclude that ifnar $1 \%$ microglia have an intrinsically impaired production, their IFN $\beta$ release after stimulation with Re-LPS, S-LPS or 
A

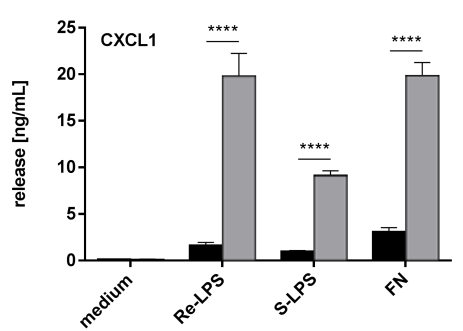

B

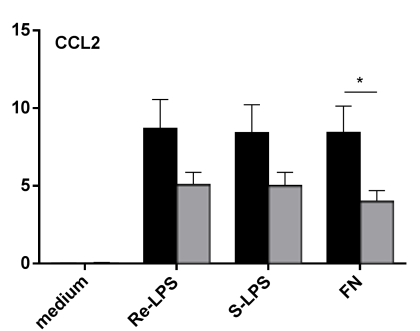

C

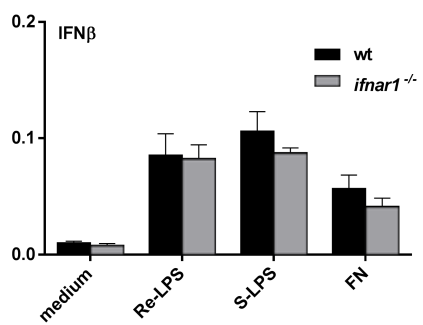

Figure 4.5: IFNAR1 deficiency causes increased CXCL1 and decreased CCL2 production by TLR4-stimulated microglia, but does not affect IFN $\beta$ levels. Cultured microglia from wt and ifnar $1^{-/}$mice were stimulated with $10 \mathrm{ng} / \mathrm{mL}$ Re-LPS, S-LPS or $100 \mu \mathrm{g} / \mathrm{mL}$ FN for $18 \mathrm{~h}$. (A) CXCL1, (B) CCL2 and (C) IFN $\beta$ release were determined in cell culture supernatants by ELISA. Data are mean \pm SEM with $n=12$ from 3 independent experiments. Statistical analysis was performed using the Mann-Whitney test, * represents $\mathrm{p} \leq 0.05,{ }^{* * \star *}$ represents $\mathrm{p} \leq 0.0001$.

FN was assessed. For all three TLR4 agonists, the IFN $\beta$ levels did not differ between ifnar $1^{-1}$ and wt microglia (Figure 4.5 C). Consequently, the massive overproduction of CXCL1 cannot be attributed to an impairment in IFN $\beta$, but is rather caused by the IFNAR1 deficiency.

\subsubsection{IFNAR1 deficiency causes excessive neutrophil infiltration into the brain of E. coli-infected mice}

CXCL1 and CCL2 are important chemokines for shaping inflammatory reactions. Their release by tissue-resident immune cells upon recognition of pathogens leads to the recruitment of neutrophils and monocytes to the site of inflammation, where these cells contribute to the elimination of the infectious agents (Wengner et al. 2008; Tsou et al. 2007). Infections with gram-negative bacteria, such as E. coli, are mainly sensed via TLR4 as it is activated by their cell wall component LPS (Hoshino et al. 1999). Within the brain, microglia serve as the tissue-resident cells that recognize bacterial infections and subsequently mount an appropriate immune response, including recruitment of peripheral immune cells. Because the neutrophil chemoattractant CXCL1 was excessively produced and the monocyte chemoattractant CCL2 was slightly less produced upon TLR4 stimulation in IFNAR1-deficient microglia in vitro, this could also have implications for the in vivo recruitment of these cells in response to pathogens.

As a model for gram-negative bacterial CNS infection, wt and ifnar $1^{\%}$ mice were intracerebrally inoculated with E. coli K1, a serotype that is associated with neonatal meningitis (Robbins et al. 1974). $24 \mathrm{~h}$ after the infection, the infiltration of monocytes and neutrophils into the brain of infected mice was analyzed by flow cytometry, because these cells represent the first line of defense that is recruited very rapidly. In order to distinguish the different cell populations, single-cell suspensions of whole brains were stained for CD11b, CD45, Ly6G and Ly6C. Based on the expression of CD11b and CD45, in-

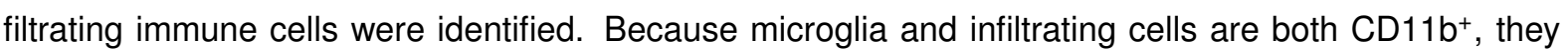
were separated by CD45 expression. Microglia express low levels and infiltrating cells high levels of CD45. For distinguishing monocytes and neutrophils within the population of infiltrating cells, the markers Ly6G and Ly6C were used. Inflammatory monocytes were considered Ly6C ${ }^{\text {high }}$ Ly6G', patrolling monocytes Ly6 $C^{\text {low }}$ Ly6G $\mathrm{G}^{-}$and neutrophils $\mathrm{Ly}_{6} \mathrm{C}^{+} \mathrm{Ly}_{6 \mathrm{G}^{+}}$(Figure 4.6A). In infected wt and ifnar ${ }^{-}$animals, recruitment of substantial amounts of inflammatory monocytes and neutrophils into the brain was observed (Figure 4.6 C and D). However, numbers of patrolling monocytes were not significantly altered by the infection in comparison to not injected animals independent of the genotype (Figure 4.6 B). Only PBS-injected mice overall showed a slight reduction in patrolling monocytes. Regarding the inflammatory monocytes, no difference between infected wt and ifnar $1 \%$ mice was observed (Figure 4.6 C). In contrast to that, IFNAR1 deficiency caused a significantly higher amount of infiltrating neutrophils 
A

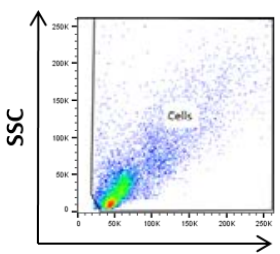

FSC

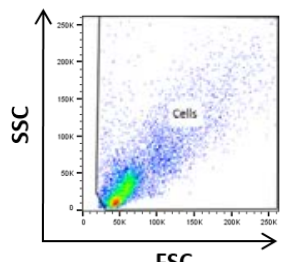

FSC

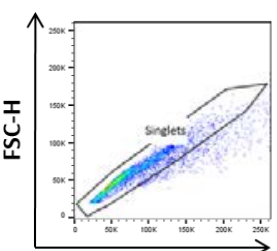

FSC-A

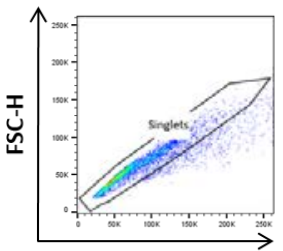

FSC-A

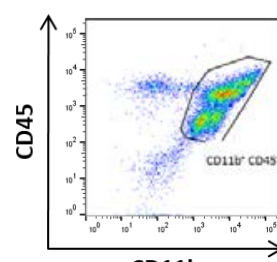

CD11b

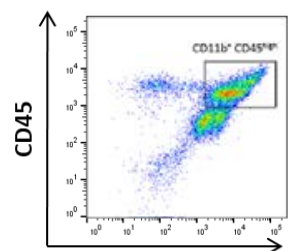

CD11b
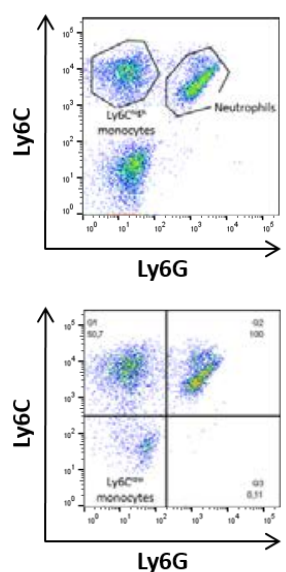

Ly6G

B

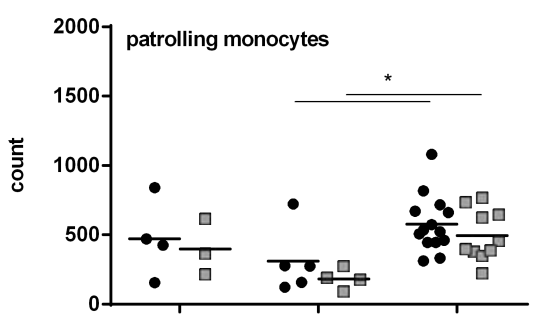

C

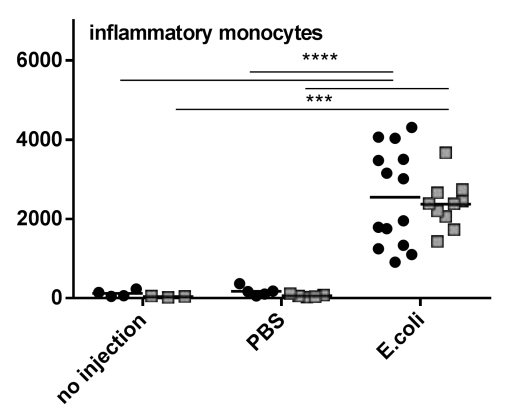

D

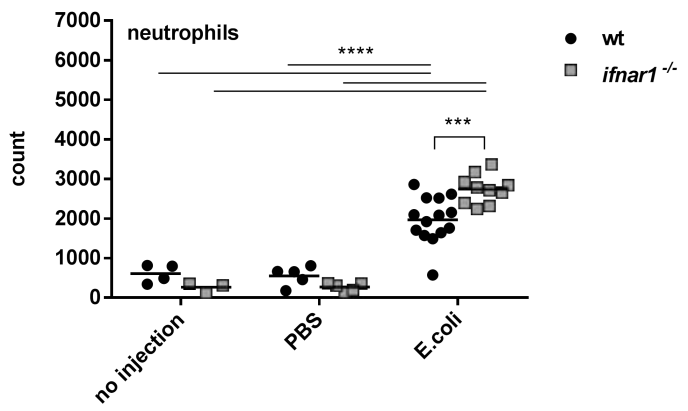

Figure 4.6: Neutrophil infiltration into the brain of E. coli-infected ifnar1 ${ }^{\%-}$ mice is higher than in wt mice. Wt and ifnar $1^{/-}$ mice were intracerebrally injected with 2000-5000 CFU E. coli K1 per mouse. As a control, mice were either injected with PBS or not injected. After $24 \mathrm{~h}$, mice were perfused with PBS. Single-cell suspensions were prepared from whole brains using the MACS Neural Tissue Dissociation Kit (T) and stained for flow cytometry as indicated. For analysis, 10,000 CD11 ${ }^{+}$cells were collected. (A) Representative flow cytometry plots showing the sequence of gating for identification of inflammatory monocytes, neutrophils and patrolling monocytes based on the expression of CD11b, CD45, Ly6G and Ly6C. (B), (C) and (D) Quantification of the cell types as described in (A). Data are individual values from 4 independent experiments. Statistical analysis was performed using the two-way ANOVA with Turkey's correction, * represents $p \leq 0.05,{ }^{* * *}$ represents $p \leq 0.001,{ }^{* * * *}$ represents $p \leq 0.0001$.

(Figure 4.6D). This can be linked to the in vitro data, showing that in the absence of IFNAR1, microglia excessively produce the neutrophil chemoattractant CXCL1. So indeed, these results have also relevance in an in vivo infection model.

\subsubsection{IFNAR1 deficiency does not impair microglial phagocytosis}

In response to pathogens and tissue damage, microglia execute a variety of different functions. For eradication of bacteria, their ability to phagocytose infectious agents was suggested to be an important feature to prevent spread of the infection (Ribes et al. 2009). If the infection is not cleared, peripheral immune cells are recruited to the CNS. This means that an impaired phagocytic capacity could lead 
to increased numbers of infiltrating cells. Because IFNAR1-deficient mice, which were intracerebrally infected with E. coli, showed an increased recruitment of especially neutrophils into the brain, it had to be ruled out that this is caused by impaired microglial phagocytosis.

Therefore, cultured microglia were incubated with DsRed-labeled E. coli for $2 \mathrm{~h}$. After removal of remaining, not engulfed bacteria by antibiotics, microglia were processed for flow cytometry analysis. They were stained for CD11b as pan-populational marker and the percentage of DsRed ${ }^{+}$cells was analyzed. As shown in Figure $4.7 \mathrm{~A}$, about $80 \%$ of the cells phagocytosed E. coli within the $2 \mathrm{~h}$. This was the case for wt as well as ifnar $1 \%$ microglia. Thus, impaired phagocytosis could not be causative for increased neutrophil recruitment in E. coli-infected ifnar $1^{-/}$mice.

A

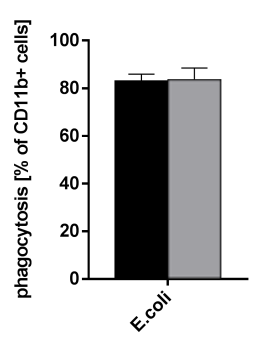

B

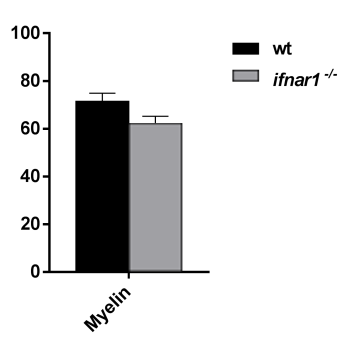

Figure 4.7: The phagocytic capacity does not differ between wt and ifnar1\% microglia. Cultured microglia from wt and ifnar $1^{-/-}$mice were were challenged with (A) $2 \times 10^{6} \mathrm{CFU} / \mathrm{mL}$ DsRed-labeled E. coli or (B) $10 \mu \mathrm{g} / \mathrm{mL}$ DyLight 550-labeled myelin for $2 \mathrm{~h}$. Subsequently, cells were stained for flow cytometry with an APC-anti-CD11b antibody. For data analysis, 10,000 CD11 ${ }^{+}$ events were included. The graphs show the phagocytosis rate as percentage of CD11 ${ }^{+}$cells. Data are mean $\pm S E M$ with $n=6$ from 3 independent experiments. Statistical analysis was performed using the Mann-Whitney test.

In addition to infectious agents, microglia are also able to phagocytose endogenous material. This includes myelin under homeostatic, but also pathological conditions, such as demyelinating diseases. As the phagocytosis of bacteria was not altered by IFNAR1 deficiency, myelin phagocytosis would most probably also not be affected. To test this, microglia were incubated with DyLight 550-labeled myelin for $2 \mathrm{~h}$ and the phagocytosis rate was determined by flow cytometry. Comparable to E. coli, myelin was phagocytosed by about $70 \%$ of all CD11 $\mathrm{b}^{+}$microglia and this was not changed in the absence of IFNAR1 (Figure 4.7B). This shows that in both genotypes, microglia have the same capacity for the phagocytosis of pathogens and endogenous material.

\subsubsection{Neutrophil infiltration in a model of focal NMO is independent of IFNAR1}

Neuromyelitis optica (NMO) is an inflammatory demyelinating disease of the CNS, characterized by optic neuritis and transverse myelitis (Kimbrough et al. 2012). The pathogenesis of this disease is closely linked to the presence of specific NMO-antibodies (NMO-Ab) (Lennon et al. 2004) that target aquaporin 4, a water channel located in astrocytic endfeet (Lennon et al. 2005). Pathologically, NMO presents with lesions in the CNS that show extensive demyelination and astrocyte loss. Inflammatory infiltrates in these lesions consist of monocytes, macrophages, lymphocytes and polymorphonuclear cells, such as neutrophils, basophils and eosinophils (Lucchinetti et al. 2002). The infiltration of polymorphonuclear granulocytes into NMO lesions in different animal models was shown to occur within $24 \mathrm{~h}$. In a rat model of focal NMO, in which circumscribed astrocyte-depleted lesions were achieved by stereotactic injection of human NMO-Ab together with human complement, granulocytes were not detected $1 \mathrm{~h}$ or $3 \mathrm{~h}$, but $24 \mathrm{~h}$ after lesion induction (Wrzos et al. 2014). Similar results were also obtained in a mouse model (Winkler, unpublished). Hence, this model of focal NMO shares some features with the previously described E. coli infection model. Both are inflammatory conditions that lead to the infiltration of neutrophils into the CNS. This recruitment is achieved in response to the respective causing agent 
within $24 \mathrm{~h}$. Because the number of infiltrating neutrophils in the E. coli infection model was IFNAR1 dependent (see chapter 4.2.2), this could be also the case in the focal NMO model.

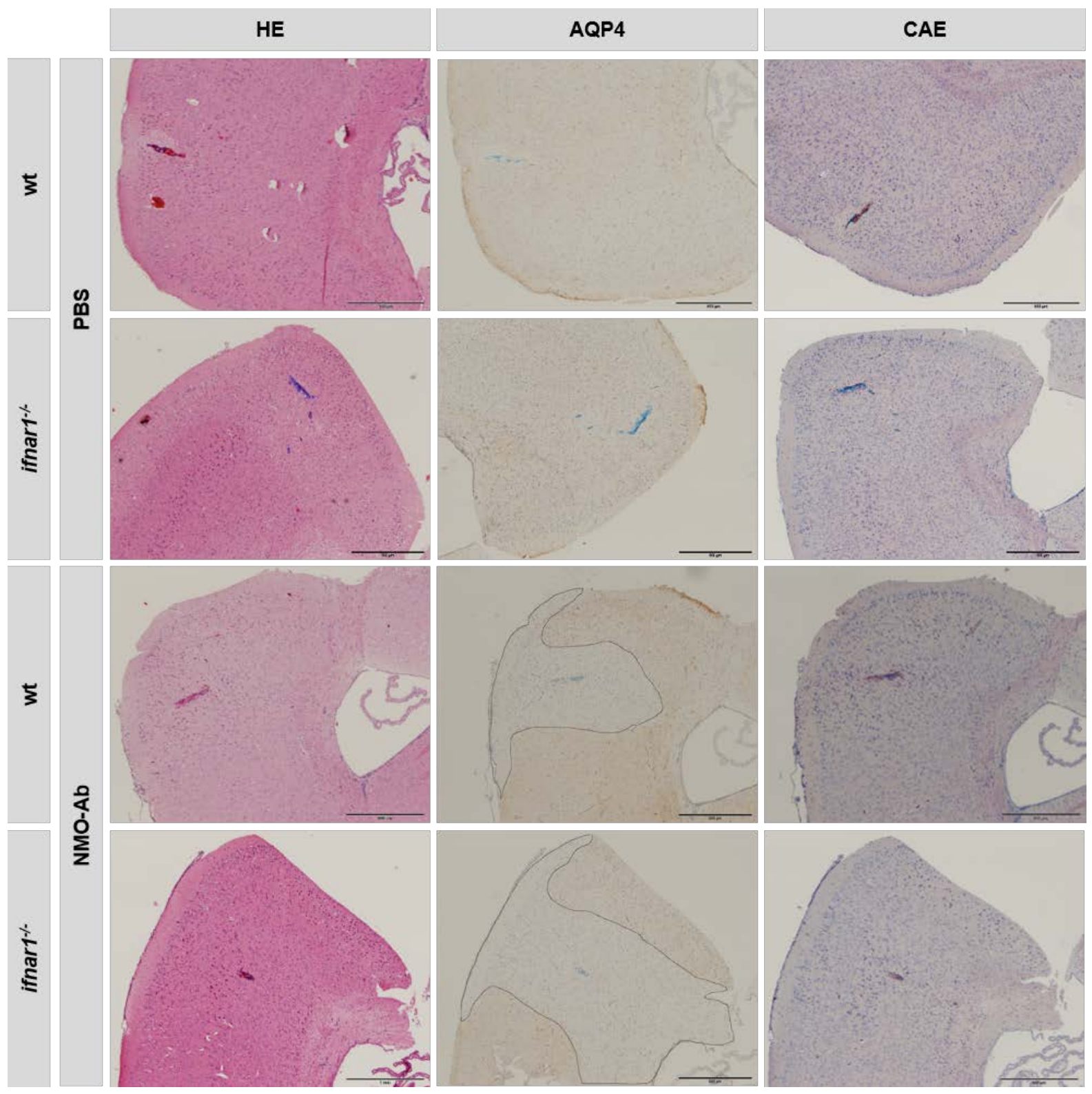

Figure 4.8: Intracortical injection of NMO-Ab together with human complement leads to the formation of astrocytedepleted lesions and infiltration of neutrophils. Wt and ifnar $1^{-1}$ mice were intracortically injected with $1 \mu \mathrm{L}$ antibody mixture, containing $15 \mathrm{U} / \mathrm{mL}$ human complement and $2.5 \mathrm{mg} / \mathrm{mL}$ recombinant anti-AQP4 antibody (NMO-Ab), or $1 \mu \mathrm{L}$ PBS as a control into both hemispheres. After 24h, mice were perfused with PBS and one hemisphere was used for histological analysis. Representative light microscopy images of HE-, AQP4- and CAE-stained sections are shown. AQP4 is stained in brown and CAE in pink. Monastral blue marks the injection site. Solid lines indicate the area of astrocyte loss. Scale bar $1 \mathrm{~mm}$

Accordingly, the neutrophil recruitment in the focal NMO model was analyzed in detail. For induction of focal NMO-like lesions, wt and ifnar $1 \%$ mice received a stereotactic injection of human NMO-Ab together with human complement into the cortex. PBS-injected mice served as control. $24 \mathrm{~h}$ after lesion induction, a histological analysis was performed. This included HE staining for a general overview of the tissue morphology and the inflammation, AQP4 staining in order to detect astrocyte-depleted lesions and CAE staining for specific identification of neutrophils in the tissue. As shown in Figure 4.8, lesions were only present in animals injected with NMO-Ab and human complement and this lesion formation was accompanied by infiltration of neutrophils. In order to compare neutrophil numbers in wt and ifnar ${ }^{\%}$ mice, all CAE positive cells in the area around the injection site were counted using a light 
microscope with an ocular morphometric grid. This revealed no difference between both genotypes (Figure 4.9B).

A
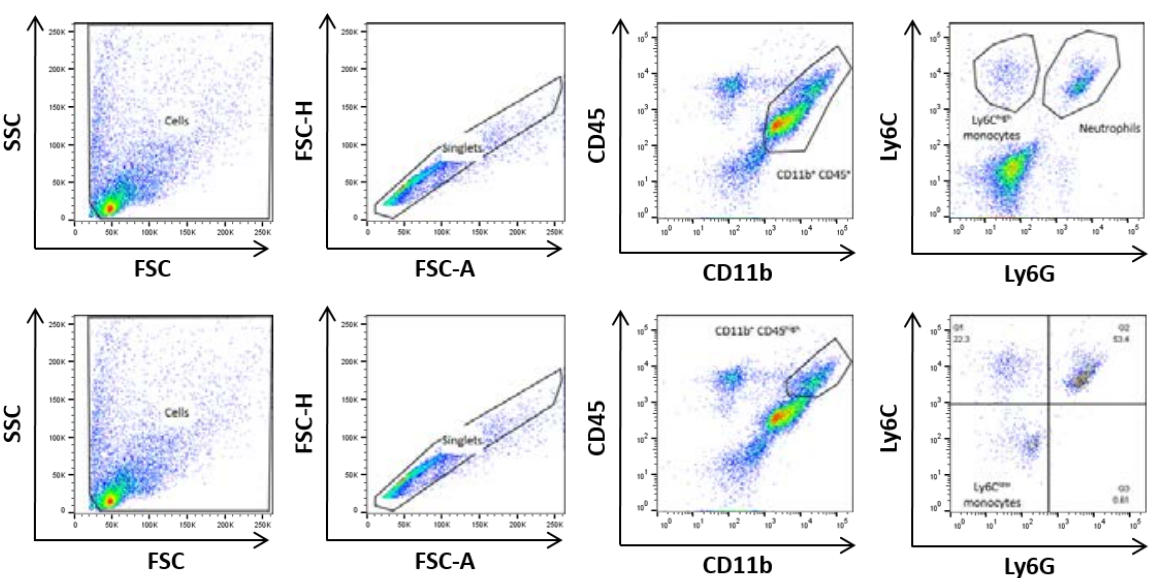

B
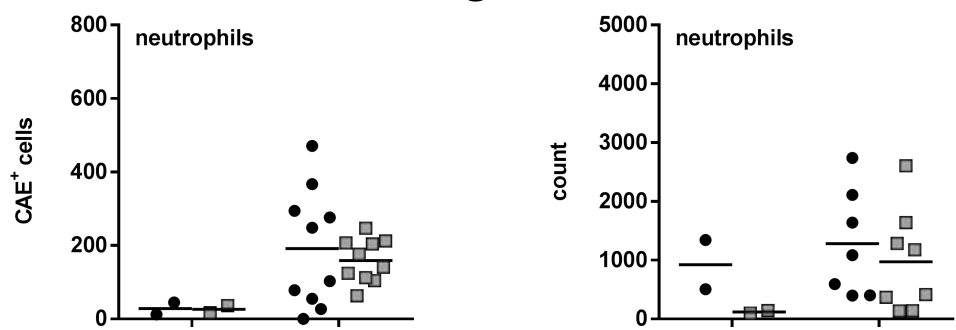

- wt

․ ifnar1 $^{-}$

D

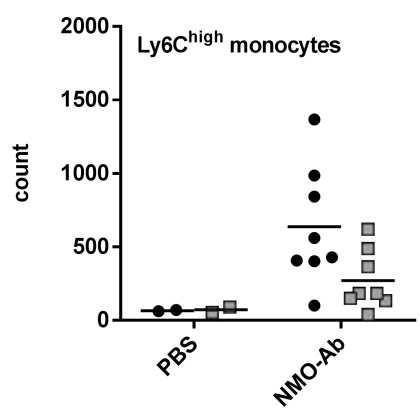

$\mathbf{E}$

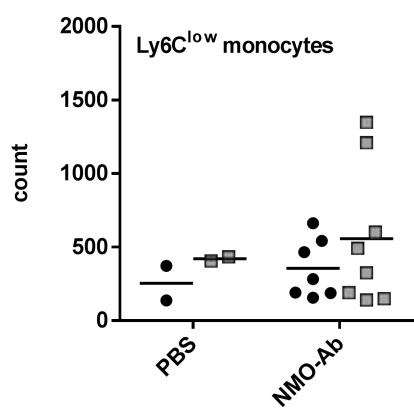

Figure 4.9: Neutrophil infiltration into focal NMO-like lesions is not affected by IFNAR1 deficiency. Wt and ifnar $1 \%$ mice were intracortically injected with $1 \mu \mathrm{L}$ antibody mixture, containing $15 \mathrm{U} / \mathrm{mL}$ human complement and $2.5 \mathrm{mg} / \mathrm{mL}$ recombinant anti-AQP4 antibody (NMO-Ab), or $1 \mu \mathrm{L}$ PBS as a control into both hemispheres. After $24 \mathrm{~h}$, mice were perfused with PBS. Singlecell suspensions were prepared from one hemisphere using the MACS Neural Tissue Dissociation Kit ( $T$ ) and stained for flow cytometry as indicated. For analysis, $10,000 \mathrm{CD} 11 \mathrm{~b}^{+}$cells were collected. (A) Representative flow cytometry plots showing the sequence of gating for identification of inflammatory monocytes, neutrophils and patrolling monocytes based on the expression of CD11b, CD45, Ly6G and Ly6C. (B) Quantification of neutrophil numbers based on the CAE staining. All CAE ${ }^{+}$cells in the area around the injections site were counted at a 20x magnification in the stained sections. (C), (D) and (E) Quantification of the cell types as described in (A). Data are individual values from 3 independent experiments. Statistical analysis was performed using the two-way ANOVA with Turkey's correction.

To confirm this result, a flow cytometry analysis similar to that described in chapter 4.2 .2 for the E. coli infection model was performed. In this case, not only the infiltrating neutrophils (referred to as CD11 $\mathrm{b}^{+}$ CD45 high $L y 6 C^{+}$Ly6G $^{+}$), but also numbers of inflammatory monocytes (referred to as CD11 b+ CD45 high

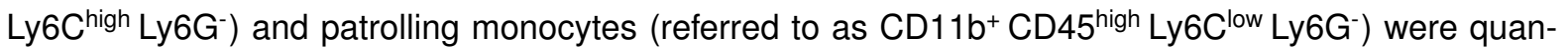
tified (Figure 4.9A). Even though more neutrophils were detected in mice injected with NMO-Ab and human complement than in PBS controls, no difference between wt and ifnar $1^{-/}$mice was observed 
(Figure $4.9 \mathrm{C}$ ). The same also applied to the inflammatory monocytes (Figure $4.9 \mathrm{D}$ ). Numbers of patrolling monocytes were neither changed by injection of NMO-Ab and human complement nor showed differences between both genotypes (Figure 4.9E). Overall, this data indicates that the infiltration of neutrophils into focal NMO-like lesions is completely independent of IFNAR1.

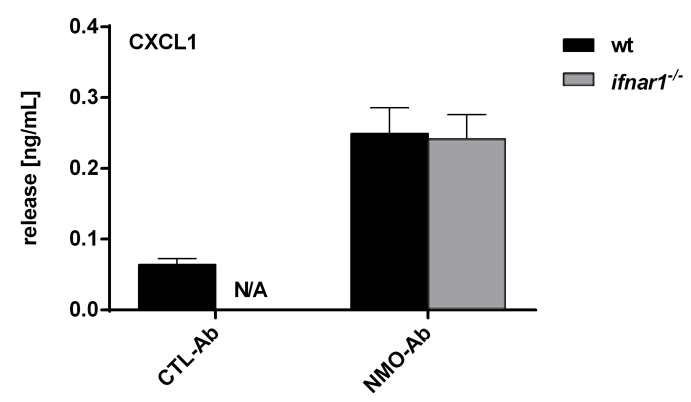

Figure 4.10: Intracerebral CXCL1 production is similarly induced in wt and ifnar1 ${ }^{/-}$mice with focal NMO-like lesions. Wt and ifnar $1 \%$ mice were intracortically injected with $1 \mu \mathrm{L}$ antibody mixture, containing $15 \mathrm{U} / \mathrm{mL}$ human complement and $2.5 \mathrm{mg} / \mathrm{mL}$ of either recombinant anti-AQP4 antibody (NMO-Ab) or recombinant 2B4 antibody (CTL-Ab), into both hemispheres. After 24h, mice were perfused with PBS. Brain lysates were prepared from one hemisphere and CXCL1 release was determined by ELISA. Data are mean \pm SEM with $n=2-4$ from 1 experiment. N/A means data not available. Statistical analysis was performed using the Mann-Whitney test.

Because the excessive neutrophil infiltration in E. coli-infected ifnar $1 \%$ mice correlated with the excessive production of the neutrophil chemoattractant CXCL1 by ifnar $1 \%$ microglia, intracerebral CXCL1 levels of mice with focal NMO-like lesions could also mirror neutrophil numbers. For that reason, wt and ifnar $1 \%$ mice were either injected with human NMO-Ab or with a CNS non-specific human antibody directed against measles virus nucleocapsid protein (CTL-Ab) together with human complement. $24 \mathrm{~h}$ after the injection, brain lysates were prepared and CXCL1 was measured by ELISA. Figure 4.10 shows that indeed CXCL1 levels are elevated in NMO-Ab-injected compared to CTL-Ab-injected animals. Unfortunately, only CTL-Ab-injected wt mice were available. Nevertheless, this increase in CXCL1 production matches the increase in neutrophil infiltration upon injection of NMO-Ab, which was observed in the histological and flow cytometry analysis (Figure 4.9B and C). In line with the data on neutrophil numbers, there was no difference in CXCL1 levels of NMO-Ab-injected wt and ifnar $1 \%$ mice. This shows that also in this model, the production of CXCL1 and recruitment of neutrophils into the brain follow a similar pattern.

\subsubsection{IFNAR1-deficient mice display lower $\mathrm{T}$ cell numbers in the brain}

When analyzing infiltrating cells in the brains of ifnar $1^{-/}$and wt mice by flow cytometry, it became obvious that also a population of $\mathrm{T}$ cells can be observed. This population was characterized as CD11b- CD45 high $\mathrm{CD}^{+}$(Figure 4.11 A). To get an overall impression, T cell counts in wt and ifnar1 ${ }^{--}$ mice were compared in the context of several treatments. This included animals, which received either no injection or an injection of PBS, E. coli or NMO-Ab together with human complement.

A comparison of $T$ cell counts in both genotypes $24 \mathrm{~h}$ after the treatment revealed significantly lower numbers in IFNAR1-deficient mice under all conditions (Figure 4.11B). Only untreated animals did not differ in their T cell numbers. Thus, IFNAR1-deficient mice display lower T cell numbers in the brain following several treatments. 
A
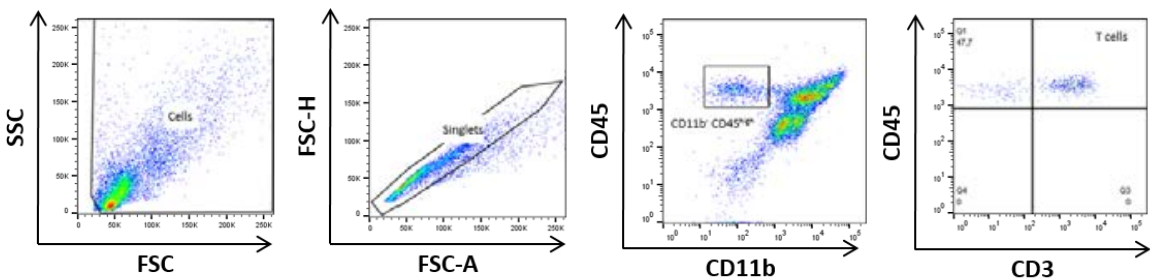

B

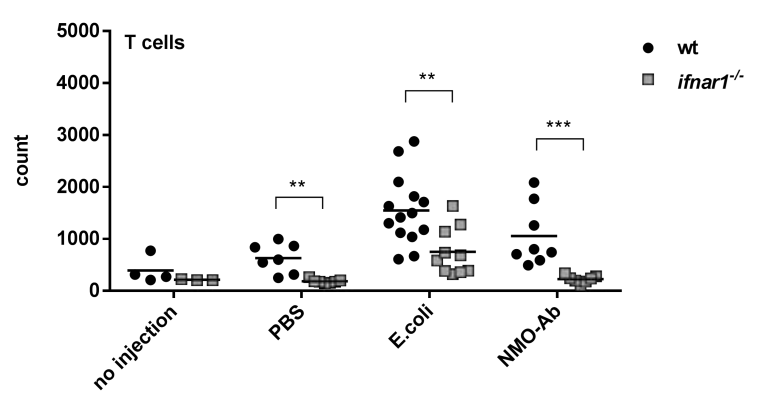

Figure 4.11: IFNAR1-deficient mice display lower $T$ cell numbers in the brain following different treatments. Wt and ifnar1 ${ }^{\%}$ mice were intracerebrally injected with $10 \mu \mathrm{L}$ PBS or 2000-5000 CFU E. coli K1 or intracortically injected with $1 \mu \mathrm{L}$ antibody mixture, containing $15 \mathrm{U} / \mathrm{mL}$ human complement and $2.5 \mathrm{mg} / \mathrm{mL}$ of recombinant anti-AQP4 antibody (NMO-Ab), per mouse. After $24 \mathrm{~h}$, mice were perfused with PBS. Single-cell suspensions were prepared from whole brains using the MACS Neural Tissue Dissociation Kit (T) and stained for flow cytometry as indicated. For analysis, 10,000 CD11 b+ cells were collected. (A) Representative flow cytometry plots showing the sequence of gating for identification of T cells based on the expression of CD11b, CD45 and CD3. (B) Quantification of T cells counts. Data are individual values from 3-4 independent experiments. Statistical analysis was performed using the Mann-Whitney test, ${ }^{* *}$ represents $p \leq 0.01$, ${ }^{* * *}$ represents $p \leq 0.001$.

\subsubsection{Altered chemokine production in IFNAR1-deficient microglia is not res- cued by time}

As described in chapter 4.2.1, IFNAR1 deficiency led to a dramatic dysregulation of microglial CXCL1 release in response to TLR4 agonists. Furthermore, it caused excessive neutrophil recruitment into the brain of E. coli-infected mice (see chapter 4.2.2). This indicates that proper regulation of CXCL1 might be crucial for mounting appropriate immune responses. In order to understand the IFNAR1-dependent control of the production of chemokines, ifnar $1^{-/}$microglia were further characterized concerning their CXCL1 and CCL2 release. As previously described, CXCL1 was massively overproduced, while CCL2 was slightly less produced in IFNAR1-deficient microglia $18 \mathrm{~h}$ after stimulation with different TLR4 agonists (see Figure 4.5). However, for a complete dependence of these factors on the presence of IFNAR1, this effect needs to be permanent and not restricted to only one time point.

To prove this, wt and ifnar1\% microglia were stimulated with Re-LPS for $48 \mathrm{~h}$ and the CXCL1 and CCL2 release was quantified over time. While a significant increase of CXCL1 levels was observed at the $16 \mathrm{~h}$ time point (Figure 4.12 A), CCL2 levels were significantly decreased in IFNAR1-deficient microglia already after $8 \mathrm{~h}$ of stimulation (Figure $4.12 \mathrm{~B}$ ). This effect was maintained during the following time and the difference between both genotypes further increased continuously. This argues for a complete dependence of the regulation of microglial CXCL1 and CCL2 production on IFNAR1. 
A

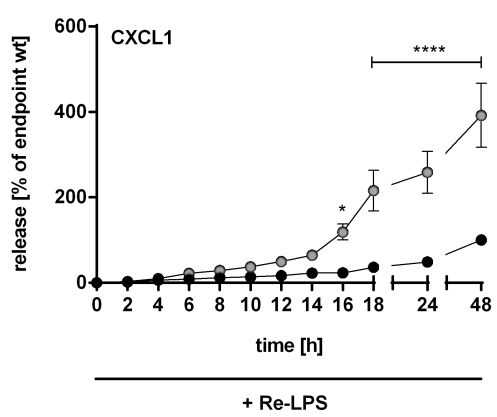

B

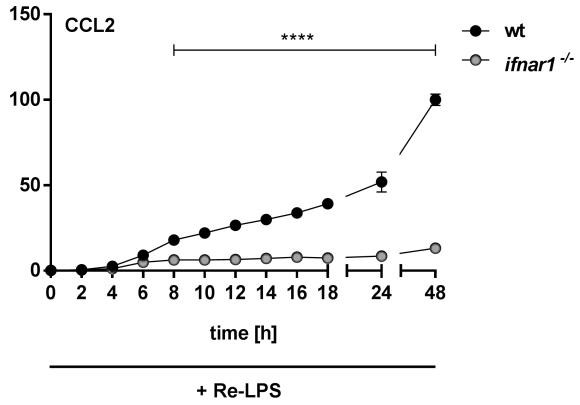

Figure 4.12: Altered CXCL1 and CCL2 production by ifnar1 ${ }^{\%}$ microglia in response to TLR4 stimulation is stable over time. Cultured microglia from wt and ifnar $1^{\%}$ mice were stimulated with $10 \mathrm{ng} / \mathrm{mL}$ Re-LPS for the indicated time points. (A) CXCL1 and (B) CCL2 release were determined in cell culture supernatants by ELISA. Absolute values were normalized to amounts produced by wt cells stimulated for $48 \mathrm{~h}$ (endpoint). Data are mean \pm SEM with $\mathrm{n}=12$ from 3 independent experiments. Statistical analysis compares both genotypes at each time point individually and was performed using the two-way ANOVA with Sidak's correction, ${ }^{\star}$ represents $p \leq 0.05,{ }^{* * * *}$ represents $p \leq 0.0001$.

\subsubsection{IFNAR1 deficiency leads to similar changes in the chemokine production of microglia and macrophages}

Because it was shown that the regulation of CXCL1 and CCL2 production by microglia depends on the presence of IFNAR1 (see chapter 4.2.1), it was investigated whether this is a cell type-specific phenomenon or similar also in other macrophages. Bone marrow-derived macrophages (BMDM) and peritoneal macrophages $(\mathrm{pM} \Phi)$ were chosen as comparative examples, because they are also tissueresident macrophages, but differ in their origin and location from microglia.
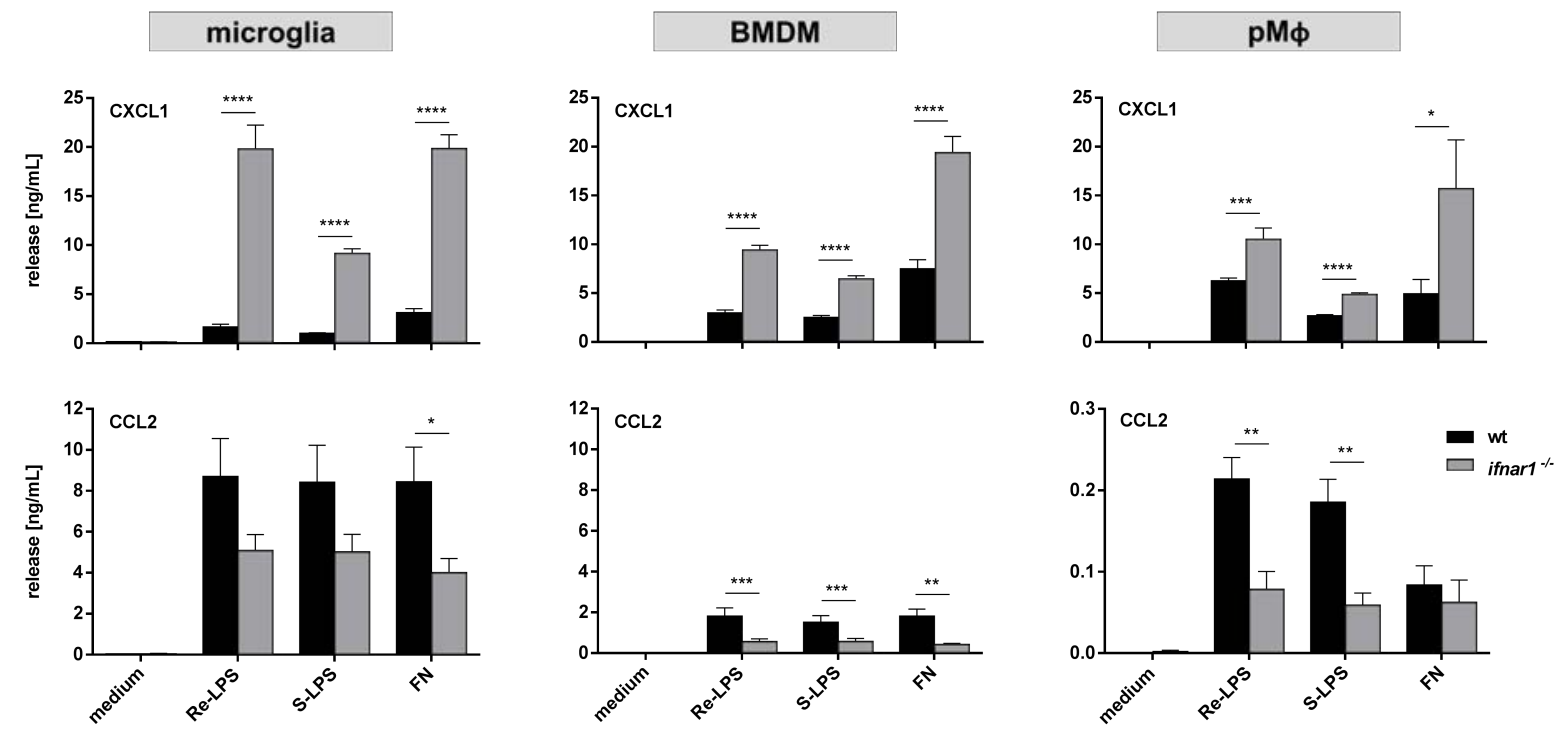

Figure 4.13: IFNAR1 deficiency similarly affects TLR4-induced CXCL1 and CCL2 production by microglia, BMDM and pM $\Phi$. Cultured microglia, BMDM and pM $\Phi$ from wt and ifnar $1^{--}$mice were stimulated with $10 \mathrm{ng} / \mathrm{mL}$ Re-LPS, S-LPS or $100 \mu \mathrm{g} / \mathrm{mL}$ $\mathrm{FN}$ for $18 \mathrm{~h}$. CXCL1 and CCL2 release were determined in cell culture supernatants by ELISA. Data are mean $\pm \mathrm{SEM}$ with $\mathrm{n}=8-$ 12 from 3-4 independent experiments. Statistical analysis was performed using the Mann-Whitney test, * represents $p \leq 0.05$, ${ }^{* *}$ represents $p \leq 0.01,{ }^{* * *}$ represents $p \leq 0.001,{ }^{* * * *}$ represents $p \leq 0.0001$.

For a comparison of these three different cell types, microglia, BMDM and $\mathrm{pM} \Phi$ from wt and ifnar 1 mice were stimulated with Re-LPS, S-LPS or FN. After $18 \mathrm{~h}$, the production of CXCL1 and CCL2 was measured by ELISA. As shown in Figure 4.13, the release pattern of all cell types was very similar. In microglia as well as BMDM and $\mathrm{pM} \Phi, \mathrm{CXCL} 1$ was massively overproduced in response to the TLR4 
agonists in the absence of IFNAR1. Furthermore, IFNAR1-deficiency led to a slightly decreased CCL2 production under all conditions, which was partially significant in microglia and $\mathrm{pM} \Phi$, but completely significant in BMDM. In summary, this comparison revealed no major differences between the three different cell types. Similar to microglia, the CXCL1 and CCL2 production was dramatically altered in ifnar $1 \%$ BMDM and $\mathrm{pM} \Phi$. This points towards a general control of IFNAR1 over CXCL1 and CCL2 production, which is not cell type-specific.

\subsubsection{IFNAR1 deficiency can be phenocopied by functional block}

The IFNAR1-deficient cells that were used for the analysis of CXCL1 and CCL2 production have been generated from mice with a global knock-out of IFNAR1. This knock-out affects the whole body already during development and could therefore cause intrinsic problems of these cells that would ultimately lead to alterations in chemokine production.

A

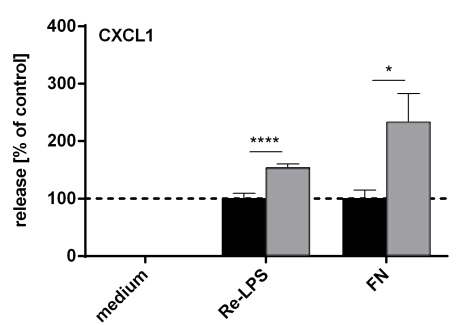

B

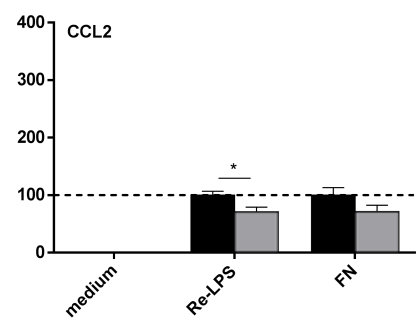

C

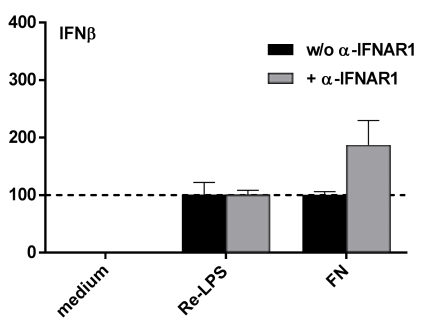

Figure 4.14: Functional block of IFNAR1 increases microglial CXCL1 and decreases CCL2 levels, but does not affect IFN $\beta$ production in response to TLR4 stimulation. Cultured microglia from wt mice were either pre-incubated (+ $\alpha$-IFNAR1) or not pre-incubated (w/o $\alpha$-IFNAR1) with $10 \mu \mathrm{g} / \mathrm{mL} \alpha$-IFNAR1 antibody for $1 \mathrm{~h}$ and afterwards stimulated with $10 \mathrm{ng} / \mathrm{mL}$ Re-LPS or $100 \mu \mathrm{g} / \mathrm{mL} \mathrm{FN}$ for $18 \mathrm{~h}$ in the presence or absence of $10 \mu \mathrm{g} / \mathrm{mL} \alpha$-IFNAR1 antibody. (A) CXCL1, (B) CCL2 and (C) IFN $\beta$ release were determined in cell culture supernatants by ELISA. Absolute values were normalized to amounts produced by cells stimulated in the absence of $\alpha$-IFNAR1 antibody. Data are mean \pm SEM with $\mathrm{n}=12$ from 3 independent experiments. Statistical analysis was performed using the Mann-Whitney test, * represents $p \leq 0.05$, ${ }^{* * *}$ represents $p \leq 0.0001$.

To rule out such a phenomenon, fully functional wt microglia were stimulated with Re-LPS or FN, while IFNAR1 was blocked by an anti $(\alpha)$-IFNAR1 antibody. The blocking antibody was applied to the cells $1 \mathrm{~h}$ before the stimulation, so that the receptor was already not functional at the time point of stimulation and was then continuously present. In the presence of $\alpha$-IFNAR1 antibody, CXCL1 production was significantly increased by $50-100 \%$ compared to the amount produced by cells stimulated in the absence of $\alpha$-IFNAR1 antibody (Figure 4.14 A). This effect was similar, but not as striking as in ifnar $1^{-/}$ microglia, where an up to twelvefold overproduction was observed (Figure 4.5). Regarding the CCL2 production, a significant reduction by $30 \%$ was achieved by IFNAR1 blockade in Re-LPS-stimulated microglia, while the slight reduction in FN-stimulated cells was not significant (Figure 4.14 B). Similar to ifnar $1^{-/}$microglia, the release of IFN $\beta$ was not significantly changed by the IFNAR1-blocking antibody (Figure $4.14 \mathrm{C}$ ). Overall, functional blockade of IFNAR1 could phenocopy the effects on chemokine production that were present in ifnar $1 \%$ microglia. This means that changes in CXCL1 and CCL2 levels were indeed only due to IFNAR1 deficiency and not caused by other, cell intrinsic alterations.

\subsubsection{Regulation of CXCL1 and CCL2 production depends on IFNAR2}

As described previously, IFNAR consists of the two subunits IFNAR1 and IFNAR2, which are both required for effective ligand binding (Cohen et al. 1995). Nevertheless, it was shown that under certain circumstances IFN $\beta$ can specifically ligate to IFNAR1 in an IFNAR2-independent manner (de Weerd et al. 2013). Because all experiments described so far have only considered IFNAR1, effects of IFNAR2 
should also be investigated. In particular, the regulation of CXCL1 and CCL2 production by IFNAR2 was subject of this analysis.

A

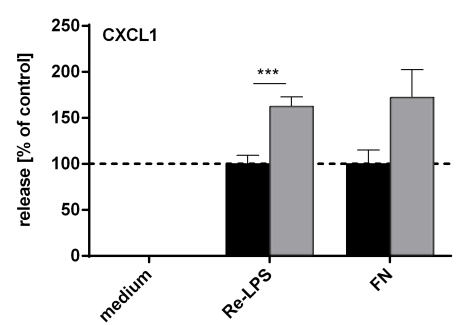

B

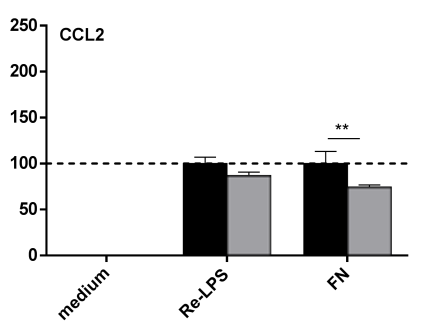

C

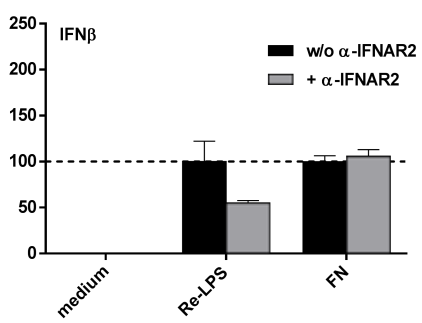

Figure 4.15: Functional block of IFNAR2 increases microglial CXCL1 and decreases CCL2 levels, but does not affect IFN $\beta$ production in response to TLR4 stimulation. Cultured microglia from wt mice were either pre-incubated (+ $\alpha$-IFNAR2) or not pre-incubated (w/o $\alpha$-IFNAR2) with $10 \mu \mathrm{g} / \mathrm{mL} \alpha$-IFNAR2 antibody for $1 \mathrm{~h}$ and afterwards stimulated with $10 \mathrm{ng} / \mathrm{mL}$ Re-LPS or $100 \mu \mathrm{g} / \mathrm{mL} \mathrm{FN}$ for $18 \mathrm{~h}$ in the presence or absence of $10 \mu \mathrm{g} / \mathrm{mL} \alpha$-IFNAR2 antibody. (A) CXCL1, (B) CCL2 and (C) IFN $\beta$ release were determined in cell culture supernatants by ELISA. Absolute values were normalized to amounts produced by cells stimulated in the absence of $\alpha$-IFNAR2 antibody. Data are mean \pm SEM with $n=12$ from 3 independent experiments. Statistical analysis was performed using the Mann-Whitney test, ${ }^{* *}$ represents $p \leq 0.01,{ }^{* * *}$ represents $p \leq 0.001$.

Microglia were treated with an $\alpha$-IFNAR2 antibody as described in chapter 4.2.8 and stimulated with Re-LPS or FN for $18 \mathrm{~h}$. The release of CXCL1, CCL2 and IFN $\beta$ after the stimulation is summarized in Figure 4.15. Similar to what was obtained with the $\alpha$-IFNAR1 antibody (Figure 4.14), IFNAR2 blockade resulted in a $60-70 \%$ increase of CXCL1 production compared to the amount produced in the absence of $\alpha$-IFNAR2 antibody (Figure 4.15A). However, this effect could be only shown to be significant in Re-LPS-stimulated microglia. In contrast to that, CCL2 levels were only affected in FN-stimulated cells, where the $\alpha$-IFNAR2 antibody decreased the release by $30 \%$ (Figure $4.15 \mathrm{~B}$ ). The production of IFN $\beta$ was again not significantly altered (Figure $4.15 \mathrm{C}$ ). So overall, the release pattern was rather similar between IFNAR1- and IFNAR2-blocked microglia. Both antibodies led to an increase of CXCL1, a decrease of CCL2 and no change in IFN $\beta$ production. However, none of the blocking antibodies resulted in alterations that were as dramatic as in the ifnar $1^{-/}$microglia, indicating a reduced potency of receptor blockade by antibody compared to complete knock-out. Nevertheless, a contribution of IFNAR2 to the regulation of microglial CXCL1 and CCL2 production could be shown. This points towards a cooperative ligand binding by IFNAR1 and IFNAR2, which further controls chemokine production.

\subsubsection{Functional absence of IFNAR1 and IFNAR2 exceeds effects of either de- ficiency}

Because the previously described results showed that IFNAR1 and IFNAR2 independently affect TLR4triggered CXCL1 and CCL2 production, both subunits of IFNAR could cooperate in controlling these chemokines. This would mean that the observed effects could be even enhanced in the absence of IFNAR1 and IFNAR2 simultaneously.

To test this hypothesis, ifnar1 ${ }^{-/}$microglia were treated with the IFNAR2-blocking antibody in order to mimic an IFNAR1/IFNAR2 double knock-out. The cells were treated with the blocking antibody as previously described and for a comparison, the $\alpha$-IFNAR2 antibody was also used in wt microglia. So overall, four different conditions were compared: fully functional IFNAR (wt), IFNAR1 not functional (ifnar $1^{-}$), IFNAR2 not functional (wt with $\alpha$-IFNAR2 antibody) and IFNAR1/IFNAR2 not functional (ifnar $1 \%$ with $\alpha$-IFNAR2 antibody). Within all groups, cells were triggered to produce CXCL1 and CCL2 by stimulation with Re-LPS or FN. The chemokine release is summarized in Figure 4.16. For a better comparison, absolute values were normalized to amounts produced by wt cells with fully functional IFNAR. Similar to what was described in chapter 4.2.9, IFNAR2-blockade increased the CXCL1 release 
A

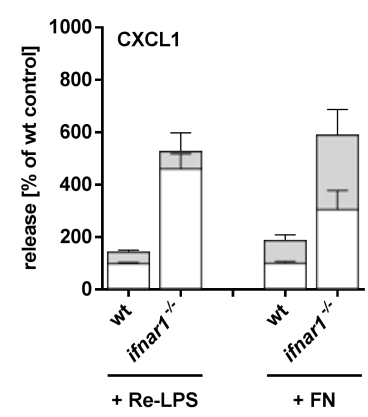

B

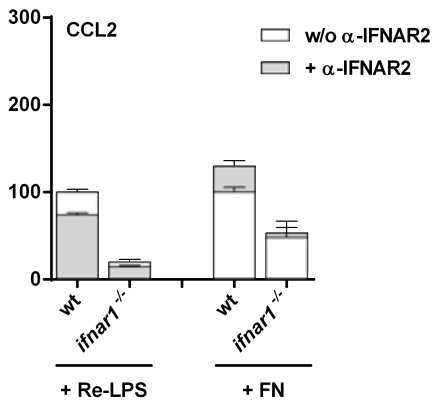

Figure 4.16: Functional absence of IFNAR1 and IFNAR2 cooperatively affects microglial CXCL1, but not CCL2 production in response to TLR4 stimulation. Cultured microglia from wt and ifnar $1 \%$ mice were either pre-incubated (+ $\alpha$-IFNAR2) or not pre-incubated (w/o $\alpha$-IFNAR2) with $10 \mu \mathrm{g} / \mathrm{mL} \alpha$-IFNAR2 antibody for $1 \mathrm{~h}$ and afterwards stimulated with $10 \mathrm{ng} / \mathrm{mL}$ Re-LPS or $100 \mu \mathrm{g} / \mathrm{mL} \mathrm{FN}$ for $18 \mathrm{~h}$ in the presence or absence of $10 \mu \mathrm{g} / \mathrm{mL} \alpha$-IFNAR2 antibody. (A) CXCL1 and (B) CCL2 release were determined in cell culture supernatants by ELISA. Absolute values were normalized to amounts produced by wt cells stimulated in the absence of $\alpha$-IFNAR2 antibody. Data are mean \pm SEM with $n=12$ from 3 independent experiments.

in wt cells by about $45-85 \%$ (Figure $4.16 \mathrm{~A}$ ). The effect of IFNAR1 knock-out was again much more striking as the CXCL1 level increased by $200-350 \%$. However, this could be even further enhanced by IFNAR2-blockade in ifnar $1 \%$ cells. IFNAR2-blockade had an additional effect of $15 \%$ on CXCL1 production in Re-LPS-stimulated ifnar ${ }^{\%}$ cells, while it even doubled the CXCL1 release by FN-stimulated ifnar $1^{-/}$cells. So indeed, functional absence of IFNAR1 and IFNAR2 simultaneously had a cooperative effect on increasing CXCL1 levels and exceeded the individual effect of either IFNAR1 or IFNAR2 deficiency. Regarding the regulation of CCL2 production, the results were not as clear. When analyzing Re-LPS-stimulated cells, the CCL2 release of wt microglia was decreased by $30 \%$ in the presence of $\alpha$-IFNAR2 antibody (Figure 4.16 B). In contrast to that, CCL2 production by wt microglia was increased by $30 \%$, when applying the $\alpha$-IFNAR2 antibody to FN-stimulated cells. However, CCL2 levels triggered by both TLR4 agonists were substantially reduced in ifnar ${ }^{-/}$microglia, resulting in less than half of the response observed in wt cells. This already low CCL2 release could not be further changed by IFNAR2blockade. Overall, this could mean that effects of the simultaneous, functional absence of IFNAR1 and IFNAR2 were not detectable, because the CCL2 levels were already as low as achievable.

\subsubsection{Regulation of CXCL1 and CCL2 production depends on janus kinases}

Tyk2 and Jak1 are tyrosine kinases that are constitutively associated with IFNAR1 and IFNAR2, respectively. Upon ligand binding to the receptor, they initiate a tyrosine phosphorylation cascade, which ultimately activates the canonical type I interferon signaling pathway (Stark et al. 1998). Because both IFNAR subunits were involved in the regulation of microglial CXCL1 and CCL2 production, it was investigated whether this effect is mediated by the two associated kinases.

In order to test the involvement of Jak1 and Tyk2 in general, microglia were treated with increasing concentrations of Jak inhibitor I, which is a reversible, ATP-competitive janus kinase inhibitor. With this compound, the activity of both kinases was blocked for $1 \mathrm{~h}$, before cells were stimulated with Re-LPS for $18 \mathrm{~h}$. During the whole stimulation period, the inhibitor was present for continuous kinase inhibition. After $18 \mathrm{~h}, \mathrm{CXCL} 1$ and CCL2 production was measured by ELISA. The release of both chemokines in dependency of the inhibitor concentration is summarized in Figure $4.17 \mathrm{~A}$. To allow a comparison of the effect on both chemokines, absolute values were normalized to amounts produced by cells that were stimulated with Re-LPS in the absence of Jak inhibitor I. With increasing concentrations of the inhibitor, CXCL1 levels continuously rose in a dose-dependent manner, while an opposing trend was observed for CCL2. CCL2 levels were significantly decreased compared to the Re-LPS-stimulated control at 
an inhibitor concentration of $100 \mathrm{nM}$. At the same concentration, the CXCL1 release was significantly above control level. With the maximum inhibitor concentration of $500 \mathrm{nM}, \mathrm{CXCL} 1$ was increased by nearly $100 \%$, while CCL2 was decreased by $75 \%$. Thus, the opposing effect of Jak inhibitor I on both chemokines was achieved dose-dependently with a similar potency and a high maximal effect.

A

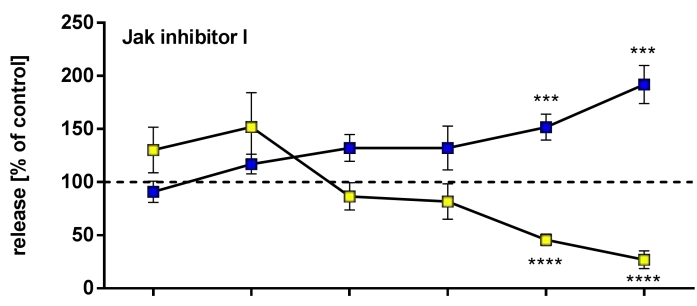

C

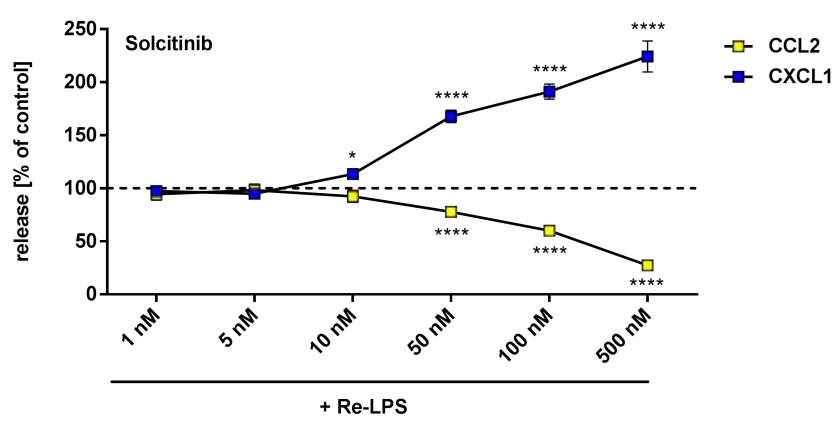

B

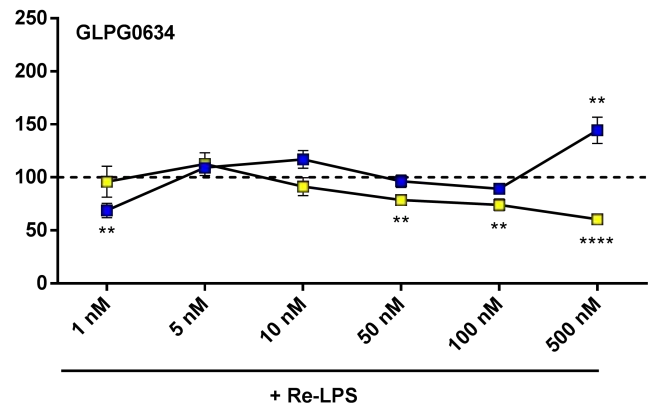

Figure 4.17: Janus kinase inhibition increases CXCL1 and decreases CCL2 production in response to TLR4 stimulation in a dose-dependent manner. Cultured microglia from wt mice were pre-incubated with the indicated doses of (A) Jak inhibitor I, (B) GLPG0634 or (C) Solcitinib for $1 \mathrm{~h}$ and afterwards stimulated with $10 \mathrm{ng} / \mathrm{mL}$ Re-LPS for $18 \mathrm{~h}$ in the presence of the respective dose of the inhibitor. CXCL1 and CCL2 release were determined in cell culture supernatants by ELISA. Absolute values were normalized to amounts produced by cells stimulated in the absence of the inhibitor. Data are mean $\pm S E M$ with $(A)$, $(B) n=12$ from 3 independent experiments or $(\mathbf{C}) n=20$ from 5 independent experiments. Statistical analysis compares the release in the presence of the respective inhibitor at the indicated concentration with the release in the absence of the inhibitor and was performed using the Mann-Whitney test, * represents $p \leq 0.05$, ${ }^{* *}$ represents $p \leq 0.01,{ }^{* * *}$ represents $p \leq 0.001$, ${ }^{* * *}$ represents $p \leq 0.0001$.

For dissecting the role of Jak1 and Tyk2 individually, an inhibitor that is more selective for Jak1, namely GLPG0634, was used. In a similar experimental setup, increasing concentrations of GLPG0634 were applied to Re-LPS-stimulated microglia. As shown in Figure $4.17 \mathrm{~B}$, this inhibitor revealed a lower potency for increasing the CXCL1 release. Only at a concentration of $500 \mathrm{nM}$, a significant increase compared to control levels was obtained. Because this was the highest used concentration, no dosedependency could be proven. Regarding the CCL2 production, GLPG0634 was slightly more potent. A significant reduction of the CCL2 release was observed at a concentration of $50 \mathrm{nM}$ and it was slightly further decreased by the presence of higher concentrations of GLPG0634. However, a maximal reduction of only $40 \%$ was achieved. Similar to that, the production of CXCL1 could be only increased by $45 \%$. In summary, GLPG0634 had a markedly lower maximal effect on CXCL1 and CCL2 production compared to Jak inhibitor I. The release of CXCL1 was less potently increased and no dose-dependency could be shown. Therefore, GLPG0634 did not serve as a good tool for investigating the role of Jak1 for the regulation of microglial chemokine production in comparison to Tyk2.

Thus, the inhibitor Solcitinib, which is selective for Jak1, was used. In contrast to GLPG0634, this compound showed to be highly potent and effective in regulating both, CXCL1 and CCL2 release. Already at a concentration of $10 \mathrm{nM}$, the CXCL1 production was significantly increased by $20 \%$ (Figure $4.17 \mathrm{C}$ ). This effect was further enhanced in a dose-dependent manner and reached a maximum of $225 \% \mathrm{com}$ pared to the baseline, representing Re-LPS-stimulated cells without any inhibitor. The regulation of 
CCL2 levels was similarly striking. Solcitinib reduced the CCL2 release by $25 \%$ at a concentration of $50 \mathrm{nM}$ and dose-dependently reached a $75 \%$ decrease at the maximum concentration of $500 \mathrm{nM}$.

Overall, inhibition of Jak1 and Tyk2 resulted in a similar outcome as IFNAR1 or IFNAR2 deficiency, an increased CXCL1 and a decreased CCL2 production by microglia in response to TLR4 stimulation. This indicates that IFNAR operates via Jak1 and Tyk2 to regulate these two chemokines. Furthermore, the use of the selective Jak1 inhibitor allowed to prove the dependence of this regulation on Jak1 specifically.

\subsubsection{Tyk2 ${ }^{E 775 K}$ microglia show properties that deviate from the previous re- sults}

By using different kinase inhibitors, it could be shown that the regulation of microglial CXCL1 and CCL2 production depends on the activity of janus kinases. In particular, the involvement of Jak1 was verified. However, not only Jak1, but also Tyk2 is activated downstream of IFNAR within the canonical type I interferon signaling pathway. Therefore, it was of interest whether Tyk2 is also important for mediating the regulatory effect of IFN $\beta$ on CXCL1 and CCL2 release. Because no specific Tyk2 inhibitor was available, a mouse strain with a naturally occurring missense mutation in the tyk2 gene (tyk2 $\left.{ }^{E 775 K}\right)$ was used in order to analyze the effect of Tyk2 deficiency.

A

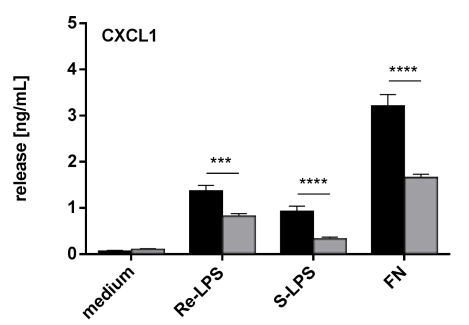

B

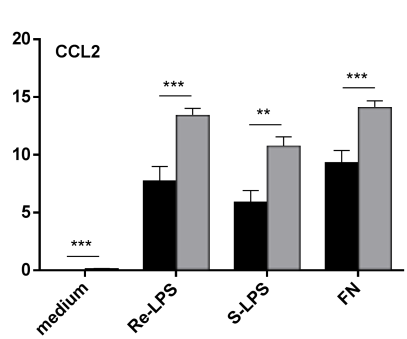

C

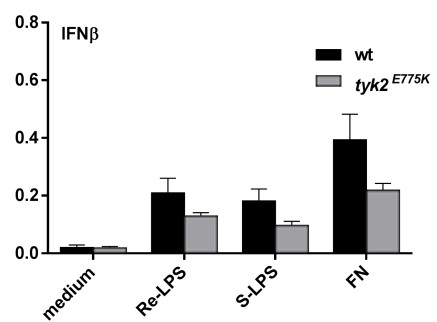

Figure 4.18: Tyk2 ${ }^{E 775 K}$ microglia produce less $C X C L 1$, more CCL2 and slightly less IFN $\beta$ than wt cells in response to TLR4 stimulation. Cultured microglia from wt and tyk2 ${ }^{E 775 K}$ mice were stimulated with $10 \mathrm{ng} / \mathrm{mL} \mathrm{Re-LPS}$, S-LPS or $100 \mu \mathrm{g} / \mathrm{mL}$ $\mathrm{FN}$ for $18 \mathrm{~h}$. (A) CXCL1, (B) CCL2 and (C) IFN $\beta$ release were determined in cell culture supernatants by ELISA. Data are mean \pm SEM with $n=16$ from 4 independent experiments. Statistical analysis was performed using the Mann-Whitney test, ${ }^{* *}$ represents $p \leq 0.01,{ }^{* * *}$ represents $p \leq 0.001,{ }^{* * * *}$ represents $p \leq 0.0001$.

Thus, microglia were isolated from these mice and their chemokine production was assessed. Following stimulation with Re-LPS, S-LPS or FN, the release of CXCL1 and CCL2 was measured by ELISA and then compared to wt cells. Because blockade of janus kinase activity resulted in a dose-dependent increase of CXCL1 levels (see Figure 4.17 A), a similar outcome was expected by using tyk2 E775K microglia. However, the complete opposite was the case. Regardless of the used TLR4 agonist, the CXCL1 production was significantly decreased in tyk2 ${ }^{E 775 K}$ cells (Figure $4.18 \mathrm{~A}$ ). They produced about half the amount that was obtained in wt cells. Regarding the CCL2 release, it was expected to be reduced in tyk2 ${ }^{E 775 K}$ microglia according to the results of janus kinase blockade that caused a dosedependent decrease of CCL2 (see Figure 4.17 A). But also in this case, the results were opposing to the expectations. In response to Re-LPS, S-LPS and FN, tyk2 ${ }^{E 775 K}$ microglia produced nearly double the amount of CCL2 that was released by wt cells (Figure 4.18B).

A possible explanation for these unexpected findings could lie in altered IFN $\beta$ levels. Therefore, IFN $\beta$ production upon TLR4 stimulation was measured in wt and tyk2 ${ }^{E 775 K}$ cells. As shown Figure $4.18 \mathrm{C}$, the production was slightly reduced in $t y k 2^{E 775 K}$ microglia. To rule out that this reduction is causative for the unexpected regulation of CXCL1 and CCL2, IFN $\beta$ levels of tyk2 ${ }^{E 775 K}$ and wt microglia were evened out by adding high amounts of external IFN $\beta$. These amounts exceeded the intrinsic microglial production 


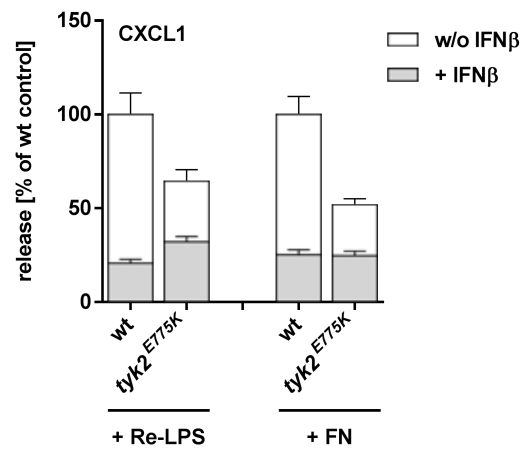

Figure 4.19: Tyk2 ${ }^{E 775 K}$ microglia are responsive to IFN $\beta$-mediated regulation of TLR4-induced CXCL1 production. Cultured microglia from wt and tyk2 ${ }^{E 775 K}$ mice were stimulated with $10 \mathrm{ng} / \mathrm{mL}$ Re-LPS or $100 \mu \mathrm{g} / \mathrm{mL}$ FN for $18 \mathrm{~h}$ in the presence $(+\operatorname{IFN} \beta)$ or absence $(\mathrm{w} / \mathrm{OIFN} \beta)$ of $10 \mathrm{ng} / \mathrm{mL}$ recombinant IFN $\beta$. CXCL1 release was determined in cell culture supernatants by ELISA. Absolute values were normalized to amounts produced by wt cells stimulated in the absence of IFN $\beta$. Data are mean \pm SEM with $\mathrm{n}=12$ from 3 independent experiments.

so much that the differences in the release of both genotypes were neglectable. Subsequently, wt and tyk2 ${ }^{E 775 K}$ microglia were stimulated with Re-LPS or FN to induce chemokine production. However, only measurements of CXCL1 could be included into the evaluation, because IFN $\beta$ itself triggered CCL2 production (data not shown) and therefore, no conclusions on TLR4-induced responses could be drawn. The results of this experiment are shown in Figure 4.19. 100\% represents the production by wt microglia in the absence of additional IFN $\beta$. If IFN $\beta$ was added to these cells, this led to a $75-80 \%$ reduction of CXCL1 upon either Re-LPS or FN stimulation. Tyk2 ${ }^{E 775 K}$ microglia produced per se only $50-60 \%$ of the CXCL1 that was obtained by wt cells. These already reduced levels could be even further decreased by adding IFN $\beta$, reaching an equal amount as compared to wt cells. Thus, tyk $2^{E 775 K}$ microglia were similarly responsive to IFN $\beta$ treatment. This argues for a still functional IFN $\beta$ regulatory feedback in these cells, even though they carry a missense mutation in tyk2.

For finally disrupting this regulatory feedback, two of the in chapter 4.2.11 described inhibitors were used. First of all, Jak inhibitor I was applied to wt and tyk2 ${ }^{E 775 K}$ microglia stimulated with Re-LPS. This inhibitor blocks Jak1 and Tyk2 meaning that, if Tyk2 was still functional in the tyk2 ${ }^{E 775 K}$ cells, its activity should be blocked by the inhibitor. Consequently, an effect on CXCL1 and CCL2 levels would be expected. But while CXCL1 was increased in a dose-dependent manner in wt cells, the tyk2 ${ }^{E 775 K}$ microglia did not react to the inhibitor (Figure 4.20 A). At all used concentrations, the CXCL1 production was only $30 \%$ of the control level, represented by Re-LPS-stimulated wt cells in the absence of the inhibitor, and could not be further increased. In contrast to that, CCL2 levels were similarly affected by Jak inhibitor I in wt and tyk2 ${ }^{E 775 K}$ microglia. Both genotypes showed a dose-dependent decrease of the CCL2 production, which was even more potent in tyk2 ${ }^{E 775 K}$ microglia, where already the lowest inhibitor concentration significantly reduced the CCL2 release (Figure $4.20 \mathrm{~B}$ ). This indicates that the regulation of CCL2 can be regained in tyk2 ${ }^{E 775 K}$ microglia by janus kinase blockade, while CXCL1 seems to be generally dysregulated.

As a comparison to the general janus kinase blockade, Solcitinib was used in the same experimental setting, because it specifically inhibits Jak1 and does not affect Tyk2. Interestingly, the pattern of CXCL1 and CCL2 release was completely the same as observed for Jak inhibitor I. Solcitinib increased the CXCL1 production only in wt, but not in tyk2 ${ }^{E 775 K}$ microglia (Figure $4.20 \mathrm{C}$ ). The release of CXCL1 in tyk2 ${ }^{E 775 K}$ cells stayed at $30 \%$ of the wt control, independent of the applied inhibitor concentration. However, the CCL2 production was dose-dependently decreased in both genotypes with a similar curve progression, but slightly higher potency in tyk2 ${ }^{E 775 K}$ microglia (Figure $4.20 \mathrm{D}$ ). So also in this case, the 
inhibitor led to a regulation of CCL2, while it failed to affect CXCL1. This argues for a generally impaired CXCL1 production in tyk2 ${ }^{E 775 K}$ microglia rather than a dysregulation of this chemokine.

A

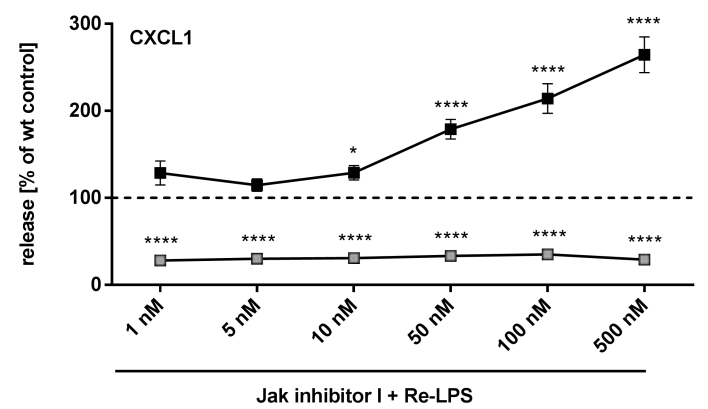

C

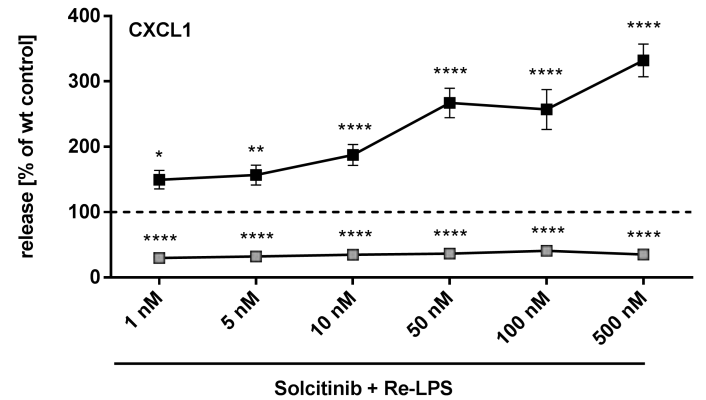

B

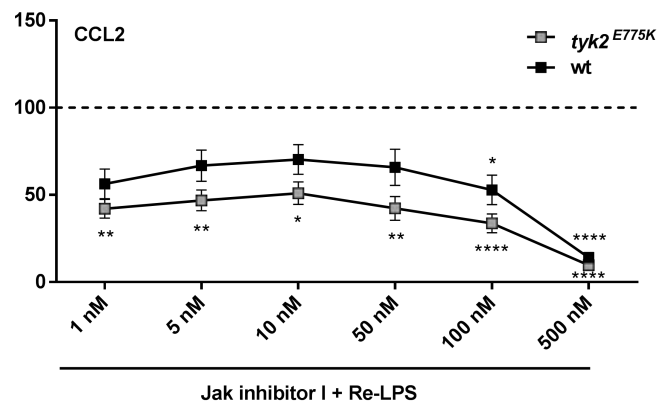

D

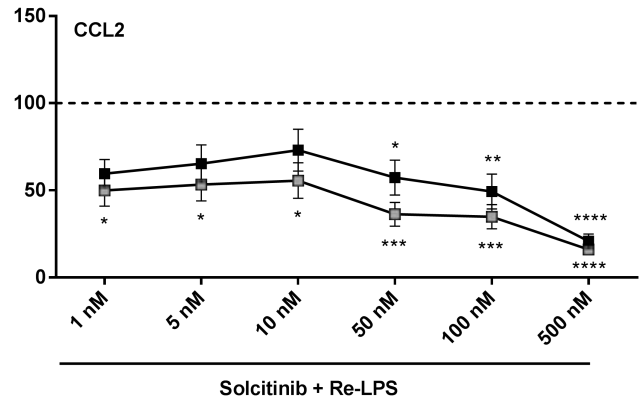

Figure 4.20: Janus kinase inhibition decreases TLR4-induced CCL2 production by tyk2 ${ }^{E 775 K}$ microglia in a dosedependent manner, but does not affect CXCL1 release by these cells. Cultured microglia from wt and tyk2 ${ }^{E 775 K}$ mice were pre-incubated with the indicated doses of (A), (B) Jak inhibitor I or (B), (C) Solcitinib for $1 \mathrm{~h}$ and afterwards stimulated with $10 \mathrm{ng} / \mathrm{mL}$ Re-LPS for $18 \mathrm{~h}$ in the presence of the respective dose of the inhibitor. (A), (C) CXCL1 and (B), (D) CCL2 release were determined in cell culture supernatants by ELISA. Absolute values were normalized to amounts produced by wt cells stimulated in the absence of the inhibitor. Data are mean \pm SEM with $n=16$ from 4 independent experiments. Statistical analysis compares the release of both genotypes stimulated in the presence of the inhibitor to the release of wt cells stimulated in the absence of the inhibitor and was performed using the Mann-Whitney test, * represents $p \leq 0.05$, ${ }^{* *}$ represents $p \leq 0.01$, ${ }^{* *}$ represents $p \leq 0.001$,

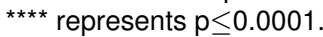

\subsubsection{Regulation of CXCL1 and CCL2 production depends on STAT1 and STAT2}

STAT proteins are intracellular transcription factors that become primarily activated by janus kinases. Within the canonical type I interferon signaling pathway, activation of Jak1 and Tyk2 results in the recruitment and phosphorylation of several STATs. The most important ones are STAT1 and STAT2, which associate with IRF9 to form the ISGF3 complex and subsequently regulate the transcription of ISGs (Platanias 2005). Because STAT1 and STAT2 are the most important mediators of type I interferon responses downstream of IFNAR and Jak1/Tyk2, they might be involved in the regulation of microglial CXCL1 and CCL2 production in response to TLR4 challenges.

A prerequisite for such an involvement would be the activation of STAT1 and STAT2 in response to TLR4 agonists. The up-regulation of both genes upon stimulation of TLR4 would already argue for that. Therefore, the expression of STAT1 and STAT2 as well as STAT3 was analyzed. Wt microglia were stimulated with Re-LPS or FN for $3 \mathrm{~h}$ and afterwards, mRNA expression was assessed by RT-PCR. The house-keeping gene gapdh served as internal control and accordingly, $\Delta \mathrm{c}_{\mathrm{T}}$ values were calculated by $\mathrm{c}_{\mathrm{T}}$ (gene of interest) - $\mathrm{c}_{\mathrm{T}}$ (gapdh). Furthermore, unstimulated cells were regarded as baseline gene expression control and so $-\Delta \Delta \mathrm{c}_{\mathrm{T}}$ values were calculated by $\Delta \mathrm{c}_{\mathrm{T}}$ (unstimulated cells) - $\Delta \mathrm{c}_{\mathrm{T}}$ (stimulated cells). Hence, values greater than 1 represent an up-regulation, while values less than -1 represent a 


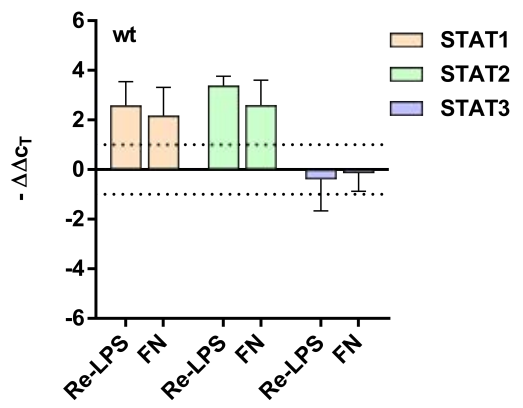

Figure 4.21: Expression of STAT1 and STAT2, but not STAT3 is up-regulated in response to TLR4 stimulation. Cultured microglia from wt mice were stimulated with $10 \mathrm{ng} / \mathrm{mL}$ Re-LPS or $100 \mu \mathrm{g} / \mathrm{mL}$ FN for $3 \mathrm{~h}$. RNA was isolated by using the RNeasy Mini Kit and converted into cDNA by using the QuantiTect ${ }^{\circledR}$ Reverse Transcription Kit. Real-Time PCR was performed by using the $\mathrm{iTaq}^{\mathrm{TM}}$ Universal SYBR ${ }^{\circledR}$ Green Supermix. Gapdh served as internal control and unstimulated cells were regarded as baseline gene expression control. The interval between the dotted lines indicates no change in gene expression. Data are mean $\pm S E M$ with $\mathrm{n}=3$ from 3 independent experiments.

down-regulation. As shown in Figure 4.21, STAT1 and STAT2 expression was up-regulated in response to Re-LPS or FN, while the expression of STAT3 was not changed. This already points towards a participation of STAT1 and STAT2 in TLR4-mediated responses.

Nevertheless, this did not necessarily refer to the regulation of TLR4-induced chemokine production. For proving such a direct relation, STAT1-deficient microglia were used. After stimulation with Re-LPS, S-LPS or FN, the release of CXCL1 and CCL2 was measured and compared to wt cells. In the absence of STAT1, the CXCL1 production was up to four times higher than in wt microglia (Figure 4.22 A). But even though a similar trend was observed for all three TLR4 ligands, a significant difference between wt

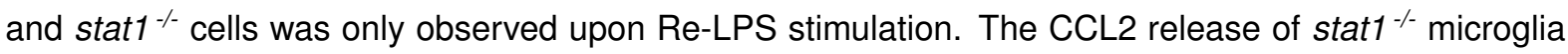
was significantly decreased under all different conditions (Figure 4.22 B).

A

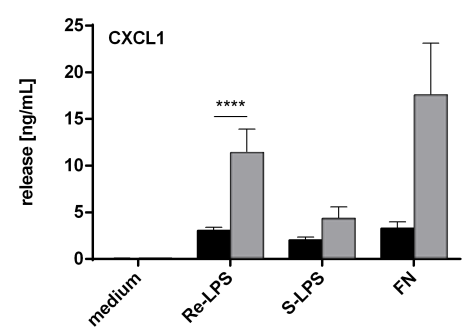

B

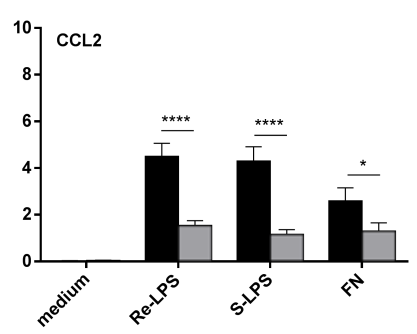

C

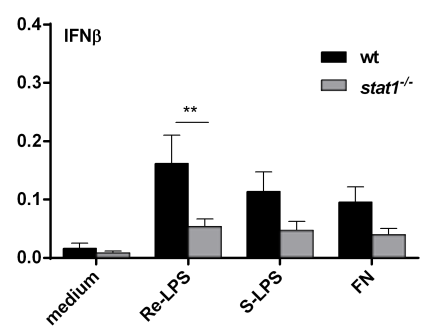

Figure 4.22: STAT1 deficiency causes increased CXCL1 and decreased CCL2 as well as IFN $\beta$ production by TLR4stimulated microglia. Cultured microglia from wt and stat $1^{\%}$ mice were stimulated with $10 \mathrm{ng} / \mathrm{mL} \mathrm{Re}-\mathrm{LPS}, \mathrm{S}-\mathrm{LPS}$ or $100 \mu \mathrm{g} / \mathrm{mL}$ FN for 18h. (A) CXCL1, (B) CCL2 and (C) IFN $\beta$ release were determined in cell culture supernatants by ELISA. Data are mean \pm SEM with $n=16$ from 4 independent experiments. Statistical analysis was performed using the Mann-Whitney test, * represents $\mathrm{p} \leq 0.05,{ }^{* *}$ represents $\mathrm{p} \leq 0.01,{ }^{* * *}$ represents $\mathrm{p} \leq 0.0001$.

To rule out that the changes in CXCL1 and CCL2 levels were caused by deficient IFN $\beta$ production in the absence of STAT1, this cytokine was also measured. However, IFN $\beta$ levels were indeed reduced in stat $^{-1}$ compared to wt microglia (Figure 4.22 C). This reduction was significant for Re-LPSstimulated cells. Thus, it needed to be dissected whether this decrease in IFN $\beta$ is causative for the altered chemokine production or just an independent observation. In order to determine that, wt and stat $1^{-}$microglia were supplemented with high amounts of IFN $\beta$ that finally led to a nearly equal level in both genotypes. Afterwards, cells were stimulated with Re-LPS or FN and the CXCL1 release was measured. This release was normalized to the amounts obtained by wt microglia in the absence of additional IFN $\beta$. In Re-LPS-stimulated wt cells, CXCL1 levels were decreased by $80 \%$ through the presence 


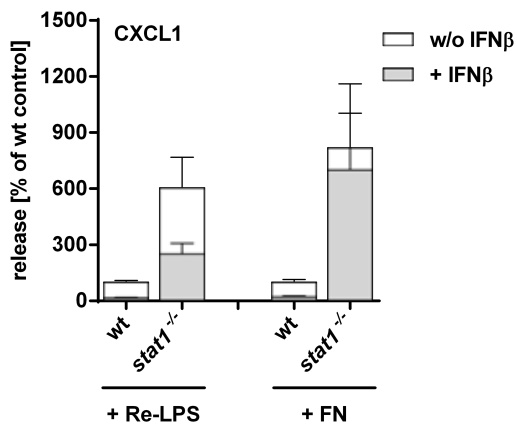

Figure 4.23: Stat1 ${ }^{\%}$ microglia are less responsive to IFN $\beta$-mediated regulation of TLR4-induced CXCL1 production than wt cells. Cultured microglia from wt and stat $1^{-/}$mice were stimulated with $10 \mathrm{ng} / \mathrm{mL}$ Re-LPS or $100 \mu \mathrm{g} / \mathrm{mL} \mathrm{FN}$ for $18 \mathrm{~h}$ in the presence $(+\operatorname{IFN} \beta$ ) or absence (w/o IFN $\beta$ ) of $10 \mathrm{ng} / \mathrm{mL}$ recombinant IFN $\beta$. CXCL1 release was determined in cell culture supernatants by ELISA. Absolute values were normalized to amounts produced by wt cells stimulated in the absence of IFN $\beta$. Data are mean \pm SEM with $n=16$ from 4 independent experiments.

of external IFN $\beta$ (Figure 4.23). Stat $1 \%$ microglia showed per se a $500 \%$ increase of CXCL1 compared to wt. This overproduction could be reduced by addition of IFN $\beta$, but it still exceeded the normal production by $150 \%$. When analyzing the FN stimulation, wt microglia behaved completely similar, but the effect in STAT1-deficient cells was even more striking. Stat $1 \%$ microglia showed a $600 \%$ increase of CXCL1 compared to wt and IFN $\beta$ treatment reduced this by less than $15 \%$. So even though stat $1 \%$ microglia were partially affected by high amounts of additional IFN $\beta$, they were much less sensitive than wt cells. This indicates that the observed changes in CXCL1 and CCL2 production are attributed to the absence of STAT1 rather than the minor IFN $\beta$ deficiency.

A

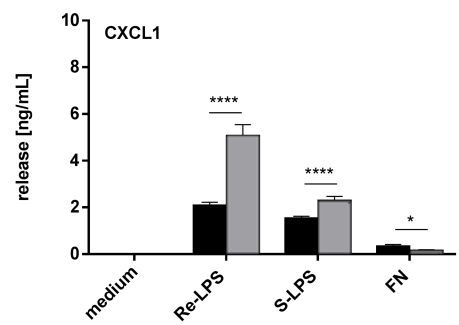

B

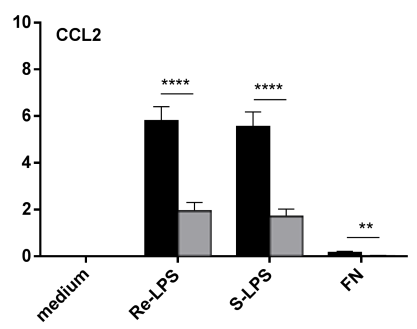

C

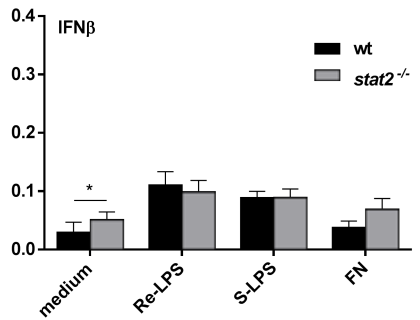

Figure 4.24: STAT2 deficiency causes increased CXCL1 and decreased CCL2 production by TLR4-stimulated microglia, but does not affect IFN $\beta$ levels. Cultured microglia from wt and stat2 $\%$ mice were stimulated with $10 \mathrm{ng} / \mathrm{mL}$ Re-LPS, S-LPS or $100 \mu \mathrm{g} / \mathrm{mL} \mathrm{FN}$ for $18 \mathrm{~h}$. (A) CXCL1, (B) CCL2 and (C) IFN $\beta$ release were determined in cell culture supernatants by ELISA. Data are mean \pm SEM with $n=24$ from 6 independent experiments. Statistical analysis was performed using the Mann-Whitney test, ${ }^{*}$ represents $p \leq 0.05,{ }^{* *}$ represents $p \leq 0.01,{ }^{* * * *}$ represents $p \leq 0.0001$.

Because STAT1 was shown to regulate CXCL1 and CCL2, it was likely that STAT2 is also involved. STAT2-deficient microglia were used in order to test this hypothesis. In a similar experiment as described above, the CXCL1 and CCL2 release by stat ${ }^{-/}$and wt cells upon TLR4 stimulation was compared. Figure $4.24 \mathrm{~A}$ shows that stat2 ${ }^{-/}$microglia produced significantly more CXCL1 in response to LPS than wt cells. The LPS-triggered CCL2 release was significantly reduced in the absence of STAT2 (Figure 4.24 B). Unfortunately, the data on FN stimulation could not be evaluated, because neither of the genotypes reacted to this FN preparation. So with regard to the CXCL1 and CCL2 levels, STAT1 and STAT2 deficiency resulted in a similar outcome.

Concerning IFN $\beta$ production, stat $1 \%$ and stat $2 \%$ microglia showed differences. While IFN $\beta$ was decreased in the absence of STAT1 (Figure $4.22 \mathrm{C}$ ), STAT2-deficiency did not alter its release (Figure $4.24 \mathrm{C}$ ). Nevertheless, the responsiveness of stat2 ${ }^{-/}$cells to high amounts of additional IFN $\beta$ was investigated. As already demonstrated, IFN $\beta$ reduced CXCL1 levels in wt cells by more than $80 \%$ (Fig- 


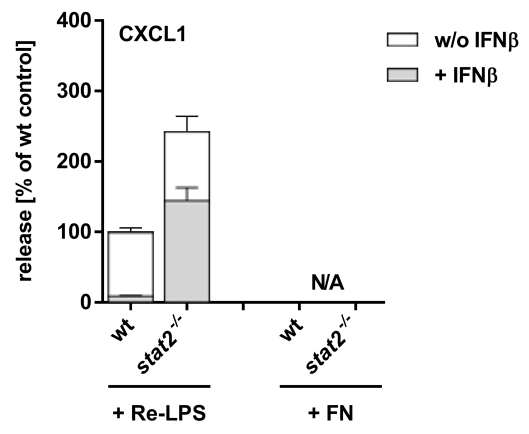

Figure 4.25: Stat2 $\%$ microglia are less responsive to IFN $\beta$-mediated regulation of TLR4-induced CXCL1 production than wt cells. Cultured microglia from wt and stat2 $\%$ mice were stimulated with $10 \mathrm{ng} / \mathrm{mL}$ Re-LPS for $18 \mathrm{~h}$ in the presence $(+$ IFN $\beta)$ or absence (w/o IFN $\beta$ ) of $10 \mathrm{ng} / \mathrm{mL}$ recombinant IFN $\beta$. CXCL1 release was determined in cell culture supernatants by ELISA. Absolute values were normalized to amounts produced by wt cells stimulated in the absence of IFN $\beta$. Data are mean \pm SEM with $\mathrm{n}=18-28$ from 7 independent experiments. N/A means data not available.

ure 4.25). However, the excessive CXCL1 production in the absence of STAT2 was decreased by only $40 \%$ through addition of IFN $\beta$ and still remained higher than the normal wt level. So similar to stat $1^{-1}$, stat $2^{-/}$microglia were only moderately responsive to IFN $\beta$ treatment.

Under normal conditions, STAT1 and STAT2 form heterodimers and jointly regulate transcription. However, the absence of either of these proteins could probably force the respective other to carry out this task autonomously. Such a phenomenon would interfere with the results obtained in $\operatorname{stat}^{\%}$ and stat ${ }^{-/}$microglia, because transcriptional regulation would be partially functional. Thus, a compensatory mechanism had to be ruled out in both knock-out strains. For that reason, microglia from STAT1and STAT2-deficient mice were stimulated with Re-LPS or FN to activate TLR4. As already described, such a stimulation led to the up-regulation of STAT1 and STAT2, but not STAT3 expression in wt cells (Figure 4.21). If a compensatory mechanism would apply, STAT2 should be up-regulated in stat $1^{-1}$ microglia and vice versa. However, this was not the case. In the absence of STAT1, STAT2 expression was not up-regulated as shown by $-\Delta \Delta \mathrm{c}_{\mathrm{T}}$ values around 1 (Figure $4.26 \mathrm{~A}$ ). The same was true for stat ${ }^{-1-}$ microglia, were no change in STAT1 expression after the stimulation was observed (Figure 4.26B). This shows that both transcription factors are cooperatively activated upon TLR4 stimulation and corresponds with the fact that stat $^{\%}$ and stat $^{\%}$ microglia had rather similar properties.

A

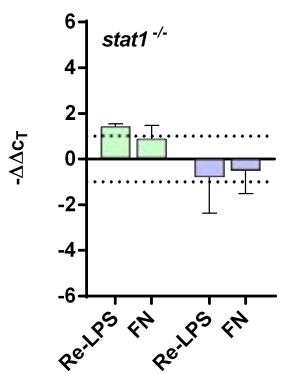

B

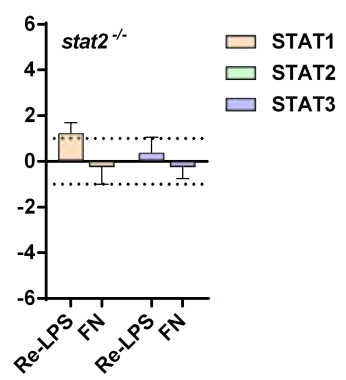

Figure 4.26: Expression of STAT1, STAT2 and STAT3 is not changed by TLR4 stimulation in stat1 ${ }^{\%}$ and stat2 ${ }^{/-}$microglia. Cultured microglia from (A) stat $1^{-1}$ and (B) stat2 $2^{-}$mice were stimulated with $10 \mathrm{ng} / \mathrm{mL}$ Re-LPS or $100 \mu \mathrm{g} / \mathrm{mL} \mathrm{FN} \mathrm{for} 3 \mathrm{~h}$. RNA was isolated by using the RNeasy Mini Kit and converted into cDNA by using the QuantiTect ${ }^{\circledR}$ Reverse Transcription Kit. Real-Time PCR was performed by using the iTaq ${ }^{\mathrm{TM}}$ Universal SYBR ${ }^{\circledR}$ Green Supermix. Gapdh served as internal control and unstimulated cells were regarded as baseline gene expression control. The interval between the dotted lines indicates no change in gene expression. Data are mean \pm SEM with $n=3$ from 3 independent experiments. 


\subsection{IFNAR controls TLR2-induced chemokine production}

Within the TLR4 system, type I interferon signaling was shown to play a crucial role in controlling microglial chemokine production. The regulation of CXCL1 and CCL2 essentially depended on an IFN $\beta$-mediated feedback that used the canonical type I interferon signaling pathway for exerting its effect. Regarding this, it was of interest whether the same regulatory mechanism applies also to other TLR systems. Because especially TLR4 responses to bacterial ligands and gram-negative infections were analyzed, another TLR that is important for fighting bacterial infections was chosen. TLR2 is essential for mounting reactions to gram-positive bacteria (Akira et al. 2006) and also able to induce the production of type I interferons under certain circumstances (Barbalat et al. 2009; Dietrich et al. 2010). Thus, regulatory effects of canonical type I interferon signaling could potentially also apply to TLR2-induced microglial responses.

\subsubsection{Regulation of CXCL1 production depends on IFNAR1}

The signaling of all type I interferons is initiated by ligand binding to IFNAR. Therefore, this receptor would be the first important mediator of an IFN-dependent regulation of microglial chemokine production in response to TLR2 activation. However, the analysis of chemokine production was in this case limited to CXCL1. In contrast to the TLR4 system, where CXCL1 and CCL2 are released upon stimulation, activation of the TLR2 receptor complex only triggers CXCL1. Microglia that were treated with Pam3CSK4 as agonist for TLR1/2 or MALP-2 as agonist for TLR6/2 did not produce any CCL2 as shown in Figure $4.27 \mathrm{~A}$.

A

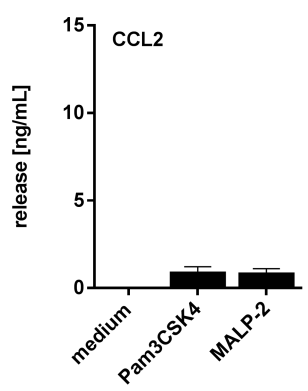

B

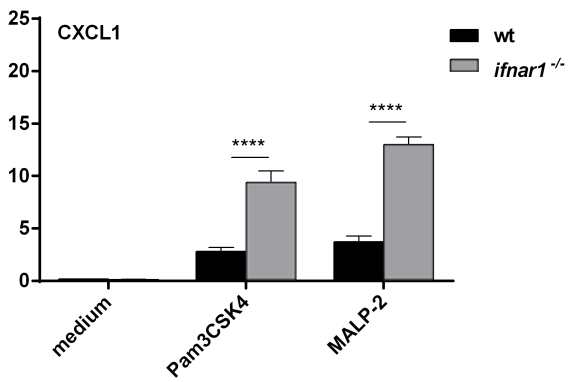

Figure 4.27: While CCL2 is in general not released in response to TLR2 stimulation, IFNAR1 deficiency causes increased CXCL1 production by TLR2-stimulated microglia. Cultured microglia from (A), (B) wt and (B) ifnar1\% mice were stimulated with $10 \mathrm{ng} / \mathrm{mL}$ Pam3CSK4 or MALP-2 for $18 \mathrm{~h}$. (A) CCL2 and (B) CXCL1 release were determined in cell culture supernatants by ELISA. Data are mean \pm SEM with $n=12$ from 3 independent experiments. Statistical analysis was performed using the Mann-Whitney test, ${ }^{* * * *}$ represents $\mathrm{p} \leq 0.0001$.

For evaluating the role of IFNAR1 in regulating CXCL1 production, reactions of wt and ifnar $1 \%$ microglia to Pam3CSK4 and MALP-2 were assessed. The comparison of both genotypes revealed differences after stimulation with both TLR2 ligands. In the absence of IFNAR1, Pam3CSK4- or MALP-2-induced CXCL1 was more than threefold increased compared to wt levels (Figure 4.27B). This indicates that IFNAR1 is indeed important for regulating TLR2-mediated CXCL1 release.

\subsubsection{IFNAR1 deficiency does not affect neutrophil infiltration into the brain of S. pneumoniae-infected mice}

S. pneumoniae is a leading cause of bacterial pneumonia, meningitis and sepsis in children worldwide. Around $11 \%$ of all deaths in children aged 1-59 months are attributed to this pathogen (O'Brien et 
al. 2009). Within the CNS, bacterial infections are recognized by the tissue-resident microglia. As a gram-positive bacterium, S. pneumoniae leads to the activation of TLR2 on these cells (Mogensen et al. 2006). In response to this activation, microglia produce chemokines and recruit peripheral immune cells, such as monocytes and neutrophils, to the site of inflammation. Because microglial production of the neutrophil chemoattractant CXCL1 upon TLR2 stimulation in vitro was increased by IFNAR1 deficiency, the attraction of neutrophils in a gram-positive bacterial infection could be enhanced accordingly.

A
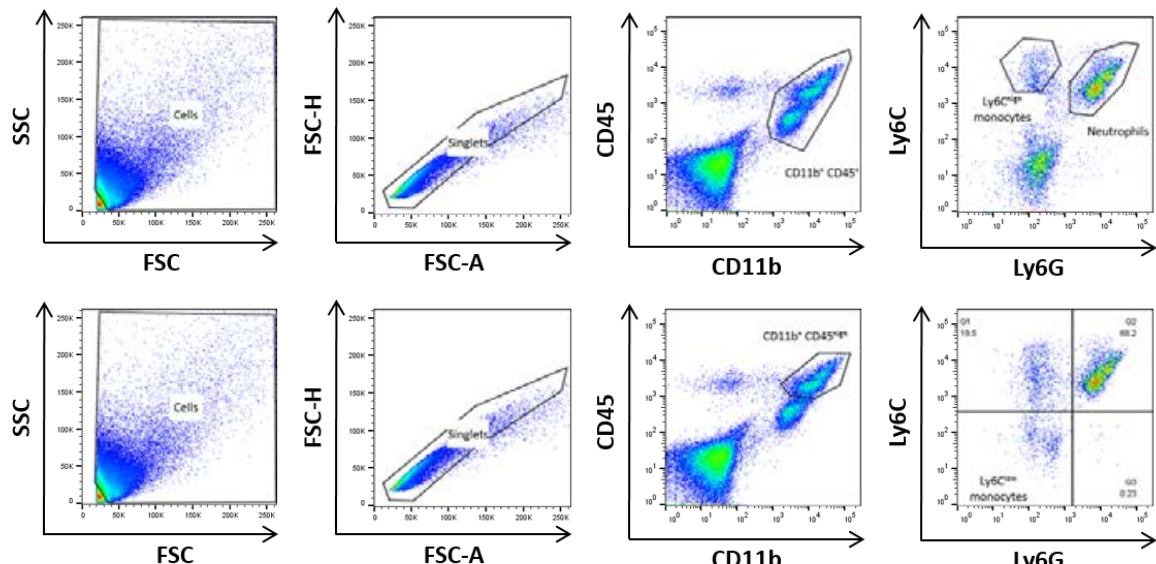

B

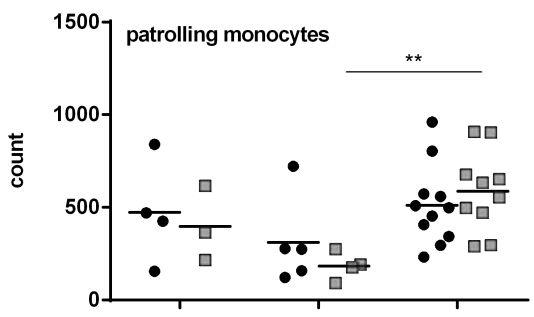

C

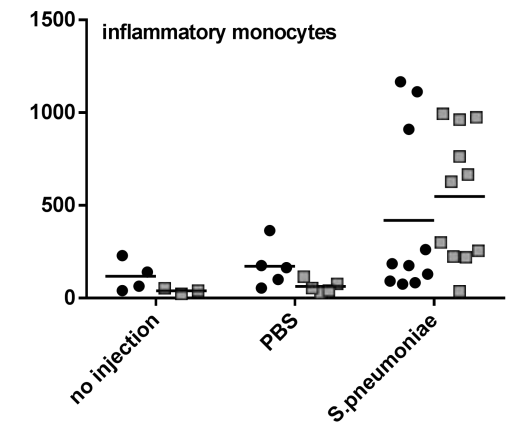

D

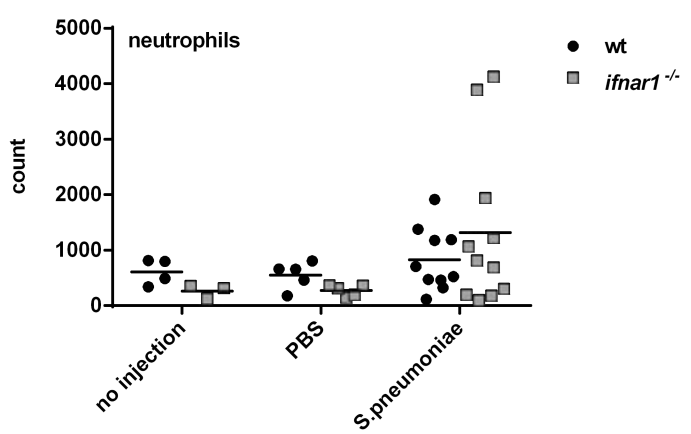

Figure 4.28: Neutrophil infiltration into the brain of S. pneumoniae-infected mice is not affected by IFNAR1 deficiency. $\mathrm{Wt}$ and ifnar $\%$ mice were intracerebrally injected with 500-800 CFU S. pneumoniae D39 per mouse. As a control, mice were either injected with PBS or not injected. After $24 \mathrm{~h}$, mice were perfused with PBS. Single-cell suspensions were prepared from whole brains using the MACS Neural Tissue Dissociation Kit $(T)$ and stained for flow cytometry as indicated. For analysis, $10,000 \mathrm{CD}_{11} \mathrm{~b}^{+}$cells were collected. (A) Representative flow cytometry plots showing the sequence of gating for identification of inflammatory monocytes, neutrophils and patrolling monocytes based on the expression of CD11b, CD45, Ly6G and Ly6C. (B), (C) and (D) Quantification of the cell types as described in (A). Data are individual values from 4 independent experiments. Statistical analysis was performed using the two-way ANOVA with Turkey's correction, ${ }^{* *}$ represents $\mathrm{p} \leq 0.01$.

To test this hypothesis, wt and ifnar1\% mice were intracerebrally infected with S. pneumoniae D39 and the subsequent infiltration of monocytes and neutrophils into the brain was assessed by flow cytometry after $36 \mathrm{~h}$. Monocytes and neutrophils were identified according to their differential expression of the markers CD11b, CD45, Ly6C and Ly6G. Inflammatory monocytes were considered CD11 b+ CD45 high 


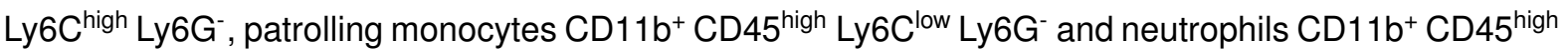
$\mathrm{Ly}_{6 \mathrm{C}^{+}} \mathrm{Ly}_{6 \mathrm{G}} \mathrm{G}^{+}$(Figure 4.28A). Regarding the amount of patrolling monocytes, no obvious systematic changes were observed in infected compared to PBS-injected or untreated animals independent of the genotype (Figure 4.28 B). Only numbers of PBS-injected and S. pneumoniae-infected ifnar ${ }^{-}$mice were significantly different. The amount of recruited inflammatory monocytes was slightly increased by the infection, but the values of single animals varied a lot, so that this increase was not significant (Figure $4.28 \mathrm{C}$ ). Nevertheless, no differences between wt and ifnar $1^{-/}$mice were observed. The same was true for the neutrophils, where the infection similarly increased the amounts in wt and ifnar $1^{-/}$animals (Figure 4.28D). Thus, IFNAR1 deficiency had no effect on the recruitment of peripheral immune cells in a model of gram-positive bacterial CNS infection.

\subsubsection{Altered chemokine production in IFNAR1-deficient microglia is not res- cued by time}

As described in chapter 4.3.1, the absence of IFNAR1 enhanced the microglial production of CXCL1 in response to TLR2 activation. The release upon stimulation with different TLR2 agonists for $18 \mathrm{~h}$ was significantly increased (Figure 4.27B). However, this does not necessarily mean that the CXCL1 production is generally dysregulated in ifnar ${ }^{\%}$ microglia. Instead, the absence of IFNAR1 could also cause a delay in the regulation, meaning that the differences in CXCL1 levels between wt and ifnar $1^{\%}$ would even out over time.

A

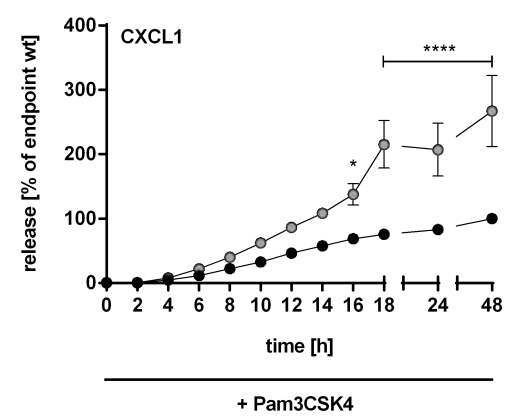

B

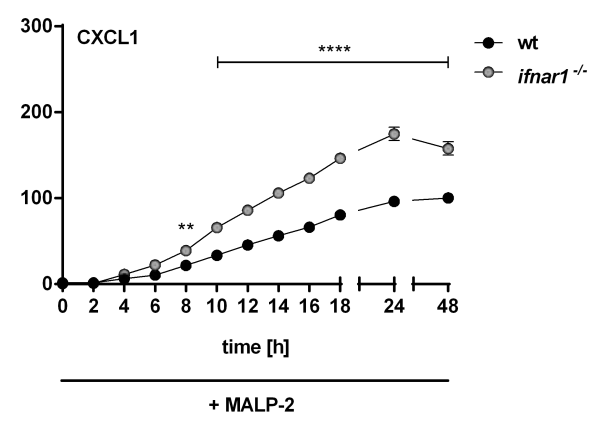

Figure 4.29: Altered CXCL1 and CCL2 production by ifnar1 ${ }^{\%-}$ microglia in response to TLR2 stimulation is stable over time. Cultured microglia from wt and ifnar $1^{-/-}$mice were stimulated with $10 \mathrm{ng} / \mathrm{mL}$ (A) Pam3CSK4 or (B) MALP-2 for the indicated time points. CXCL1 release was determined in cell culture supernatants by ELISA. Absolute values were normalized to amounts produced by wt cells stimulated for $48 \mathrm{~h}$ (endpoint). Data are mean $\pm \mathrm{SEM}$ with $\mathrm{n}=8$ from 2 independent experiments. Statistical analysis compares both genotypes at each time point individually and was performed using the two-way ANOVA with Sidak's correction, * represents $p \leq 0.05$, ${ }^{* *}$ represents $p \leq 0.01,{ }^{* * * *}$ represents $p \leq 0.0001$.

In order to rule out such a case, wt and ifnar $1^{-1-}$ microglia were stimulated with Pam3CSK4 or MALP2 for $48 \mathrm{~h}$ and the release of CXCL1 was measured over time. A significant difference between both genotypes was observed after $16 \mathrm{~h}$ of stimulation with Pam3CSK4 (Figure $4.29 \mathrm{~A}$ ) and already after $8 \mathrm{~h}$ of stimulation with MALP-2 (Figure 4.29 B). From this time point on, ifnar $1^{-/}$microglia continuously produced more CXCL1 than wt cells. With an increasing stimulation interval, CXCL1 levels always further increased in Pam3CSK4-stimulated cells of both genotypes. In contrast to that, MALP-2 stimulation induced a slightly different release pattern that in ifnar $1 \%$ microglia reached a peak after $24 \mathrm{~h}$ and afterwards already decreased again. Still, the IFNAR1-dependent increase in CXCL1 production remained constantly present in both conditions, indicating no rescue effect by time. 


\subsubsection{IFNAR1 deficiency leads to similar changes in the chemokine production of microglia and bone marrow-derived, but not peritoneal macrophages}

Microglia were shown to overproduce CXCL1 in response to Pam3CSK4 and MALP-2 in the absence of IFNAR1 (see Figure 4.27 B). Since they are the tissue-resident macrophages within the CNS, it was of interest whether a similar effect could also be observed in other tissue macrophages.
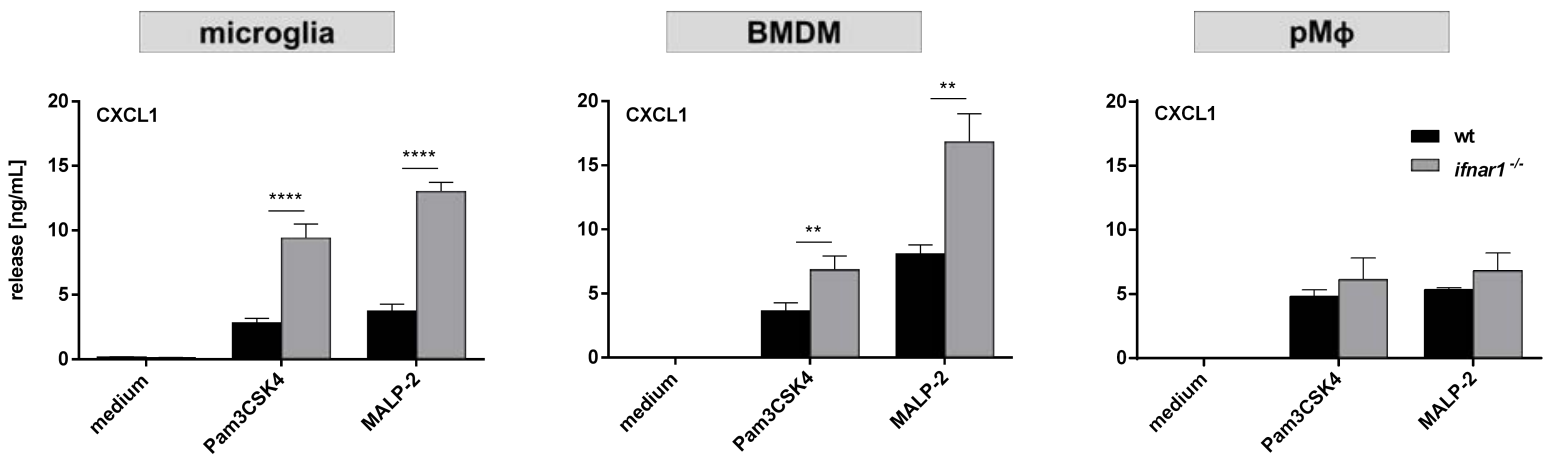

Figure 4.30: IFNAR1 deficiency similarly affects TLR2-induced CXCL1 production by microglia and BMDM, but not by pM $\Phi$. Cultured microglia, BMDM and $\mathrm{pM} \Phi$ from wt and ifnar $1^{-/}$mice were stimulated with $10 \mathrm{ng} / \mathrm{mL}$ Pam $3 C S K 4$ or MALP-2 for $18 \mathrm{~h}$. CXCL1 release was determined in cell culture supernatants by ELISA. Data are mean \pm SEM with $n=8-12$ from 3-4 independent experiments. Statistical analysis was performed using the Mann-Whitney test, ${ }^{* *}$ represents $p \leq 0.01,{ }^{* * * *}$ represents $\mathrm{p} \leq 0.0001$.

Therefore, microglia, BMDM and $\mathrm{pM} \Phi$ from wt and ifnar $1 \%$ mice were stimulated with the two TLR2 agonists and the CXCL1 release was quantified. In microglia and BMDM, CXCL1 levels were significantly enhanced in the absence of IFNAR1 (Figure 4.30). Ifnar ${ }^{\%}$ microglia showed a threefold increase and ifnar ${ }^{-1}$ BMDM a doubling compared to wt. In contrast to that, the CXCL1 production of $\mathrm{pM} \Phi$ was not altered by IFNAR1 deficiency. So overall, CXCL1 production seems not to be generally controlled by IFNAR1, but is rather differentially affected in certain cell types.

\subsubsection{IFNAR1 deficiency can be partially phenocopied by functional block}

Because so far all experiments that analyzed effects of IFNAR1 deficiency were performed using cells isolated from mice with a global IFNAR1 knock-out, the possibility of knock-out-specific impairments had to be ruled out. A good option for doing this was the comparison of ifnar $1^{-/}$cells with a functional block of IFNAR1 in wt cells.

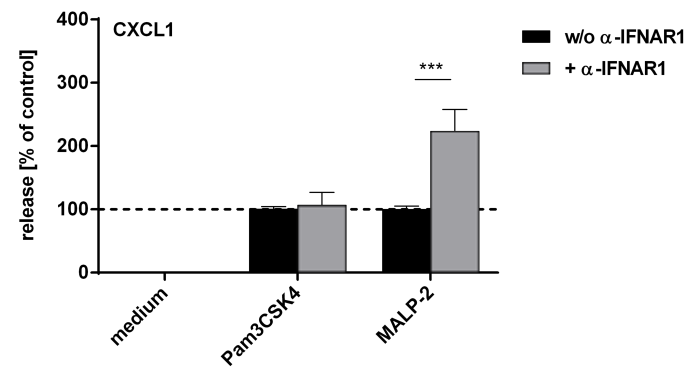

Figure 4.31: Functional block of IFNAR1 increases microglial CXCL1 production in response to MALP-2, but not Pam3CSK4 stimulation. Cultured microglia from wt mice were either pre-incubated (+ $\alpha$-IFNAR1) or not pre-incubated (w/o $\alpha$-IFNAR1) with $10 \mu \mathrm{g} / \mathrm{mL} \alpha$-IFNAR1 antibody for $1 \mathrm{~h}$ and afterwards stimulated with $10 \mathrm{ng} / \mathrm{mL}$ Pam3CSK4 or MALP-2 for $18 \mathrm{~h}$ in the presence or absence of $10 \mu \mathrm{g} / \mathrm{mL} \alpha$-IFNAR1 antibody. CXCL1 release was determined in cell culture supernatants by ELISA. Absolute values were normalized to amounts produced by cells stimulated in the absence of $\alpha$-IFNAR1 antibody. Data are mean \pm SEM with $n=12$ from 3 independent experiments. Statistical analysis was performed using the Mann-Whitney test, *** represents $\mathrm{p}<0.001$. 
For functionally blocking IFNAR1, wt microglia were treated with an $\alpha$-IFNAR1 antibody for $1 \mathrm{~h}$. Afterwards, cells were stimulated with Pam3CSK4 or MALP-2 for $18 \mathrm{~h}$ in the presence of the blocking antibody. The subsequent release of CXCL1 is summarized in Figure 4.31. Absolute values were normalized to the amount produced by wt cells that were stimulated in the absence of $\alpha$-IFNAR1 antibody. Microglial CXCL1 production in response to Pam3CSK4 was not altered by functional blockade of IFNAR1. However, CXCL1 levels following MALP-2 stimulation were more than doubled in the presence of $\alpha$-IFNAR1 antibody. This mimics the effect that was observed in ifnar $1^{\%}$ microglia, but only for MALP-2-stimulated cells.

\subsubsection{Regulation of CXCL1 production depends on IFNAR2}

The type I interferon receptor comprises not only IFNAR1, but also IFNAR2. Because thus far only the involvement of IFNAR1 in the control of TLR2-induced microglial chemokine production was demonstrated, a potential role for the other receptor subunit should be investigated.

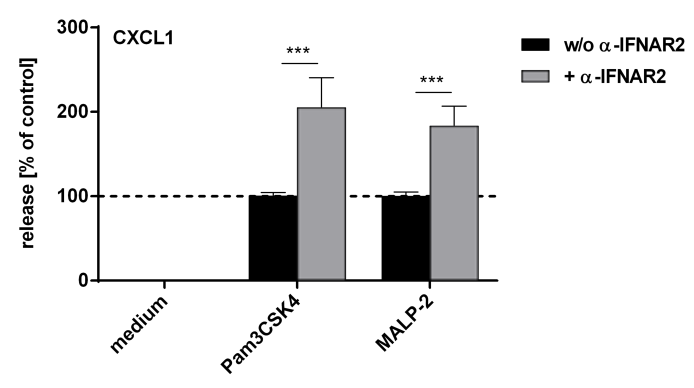

Figure 4.32: Functional block of IFNAR2 increases microglial CXCL1 production in response to TLR2 stimulation. Cultured microglia from wt mice were either pre-incubated $(+\alpha$-IFNAR2) or not pre-incubated (w/o $\alpha$-IFNAR2) with 10 $\mathrm{Mg} / \mathrm{mL} \alpha$ IFNAR2 antibody for $1 \mathrm{~h}$ and afterwards stimulated with $10 \mathrm{ng} / \mathrm{mL}$ Pam3CSK4 or MALP-2 for $18 \mathrm{~h}$ in the presence or absence of $10 \mu \mathrm{g} / \mathrm{mL} \alpha$-IFNAR2 antibody. CXCL1 release was determined in cell culture supernatants by ELISA. Absolute values were normalized to amounts produced by cells stimulated in the absence of $\alpha$-IFNAR2 antibody. Data are mean $\pm \mathrm{SEM}$ with $\mathrm{n}=12$ from 3 independent experiments. Statistical analysis was performed using the Mann-Whitney test, ${ }^{\star * *}$ represents $p \leq 0.001$.

In this regard, an $\alpha$-IFNAR2 antibody served as tool to functionally block IFNAR2 and analyze effects of IFNAR2 absence. Microglia were treated with this antibody as described in the previous chapter and the release of CXCL1 upon stimulation with Pam3CSK4 or MALP-2 was measured by ELISA. The production in the presence of $\alpha$-IFNAR2 antibody was normalized to the production in the absence of the antibody upon treatment with the respective TLR2 agonist. As shown in Figure 4.32, functional blockade of IFNAR2 significantly enhanced CXCL1 levels in response to Pam3CSK4 or MALP-2. The release was twice as high as in the absence of $\alpha$-IFNAR2 antibody. This is rather comparable to effects that were observed in ifnar $1 \%$ microglia, which produced three times more CXCL1 than wt cells (see Figure $4.27 \mathrm{~B}$ ). Consequently, both IFNAR subunits seem to cooperate in mediating the regulation of TLR2-triggered CXCL1.

\subsubsection{Functional absence of IFNAR1 and IFNAR2 exceeds effects of either de- ficiency}

As previously described, the functional absence of either IFNAR1 or IFNAR2 had an effect on the CXCL1 production. Because this suggests a cooperation of both IFNAR subunits in regulating this chemokine, a direct proof of such a fact was requested.

For simultaneous disruption of IFNAR1 and IFNAR2, ifnar $1^{-/}$microglia were treated with the $\alpha$-IFNAR2 antibody. In this case, both receptor subunits should not be functional. In comparison to that, microglia 


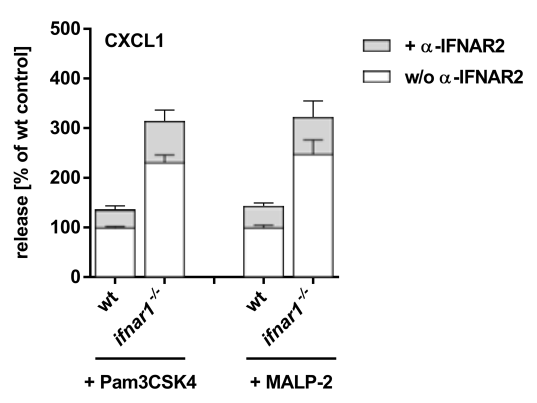

Figure 4.33: Functional absence of IFNAR1 and IFNAR2 cooperatively affects microglial CXCL1 production in response to TLR2 stimulation. Cultured microglia from wt and ifnar $1^{-/}$mice were either pre-incubated $(+\alpha$-IFNAR2) or not pre-incubated (w/o $\alpha$-IFNAR2) with $10 \mu \mathrm{g} / \mathrm{mL} \alpha$-IFNAR2 antibody for $1 \mathrm{~h}$ and afterwards stimulated with $10 \mathrm{ng} / \mathrm{mL}$ Pam3CSK4 or MALP-2 for $18 \mathrm{~h}$ in the presence or absence of $10 \mu \mathrm{g} / \mathrm{mL} \alpha$-IFNAR2 antibody. CXCL1 release was determined in cell culture supernatants by ELISA. Absolute values were normalized to amounts produced by wt cells stimulated in the absence of $\alpha$-IFNAR2 antibody. Data are mean \pm SEM with $n=12$ from 3 independent experiments.

with only IFNAR1 deficiency and solely IFNAR2-blocked microglia were used. All cells were stimulated with Pam3CSK4 or MALP-2 and the CXCL1 production was quantified after $18 \mathrm{~h}$. The $\alpha$-IFNAR2 antibody was applied to the cells $1 \mathrm{~h}$ prior to the stimulation and remained present the whole time. In order to compare the CXCL1 levels, absolute values were normalized to amounts produced by wt cells. As already described in chapter 4.3.1, IFNAR1 deficiency caused a more than $130 \%$ increase in CXCL1 production compared to wt levels (Figure 4.33). Furthermore, similar to the results shown in chapter 4.3.6, IFNAR2 blockade increased the CXCL1 release in Pam3CSK4- and MALP-2-stimulated wt microglia, in this case by $35-40 \%$. In the absence of IFNAR1 and IFNAR2 simultaneously as achieved by IFNAR2 blockade in ifnar $1^{-/}$microglia, CXCL1 levels were even further increased compared to either IFNAR1 knock-out or IFNAR2 blockade. IFNAR2-blocked ifnar $1 \%$ cells produced more than double the amount of CXCL1 than IFNAR2-blocked wt cells. Apart from that, IFNAR2 blockade had an additional effect on the already increased CXCL1 release by ifnar $1^{\%}$ cells of about $25 \%$. Overall, ifnar $1 \%$ microglia treated with the $\alpha$-IFNAR2 antibody produced nearly $350 \%$ of the normal CXCL1 release in wt cells. So in summary, functional absence of IFNAR1 and IFNAR2 resulted in an enhanced CXCL1 production that exceeded the effect of deficiency in either receptor subunit.

\subsubsection{Regulation of CXCL1 production is independent of janus kinases}

The canonical type I interferon signaling pathway, which is initiated by ligand binding to IFNAR, further uses the two tyrosine kinases Jak1 and Tyk2 for signal transduction (Stark et al. 1998). As IFNAR1 and IFNAR2 were shown to cooperatively affect microglial CXCL1 production, the involvement of both downstream kinases in this regulation was investigated.

A first overview about the general contribution of Jak1 and Tyk2 was obtained by blocking their kinase activity with increasing concentrations of Jak inhibitor I. Therefore, microglia were treated with the inhibitor $1 \mathrm{~h}$ prior to and then continuously during the stimulation with Pam3CSK4 or MALP-2 for $18 \mathrm{~h}$. The release of CXCL1 in response to both TLR2 agonists is shown in Figure 4.34 A. Regarding Pam3CSK4-stimulated cells, only a minor effect of Jak inhibitor I was observed. At a concentration of $10 \mathrm{nM}$ and $100 \mathrm{nM}, \mathrm{CXCL} 1$ levels were significantly increased compared to the amounts produced by cells that were stimulated with Pam3CSK4 in the absence of Jak inhibitor I. However, none of the other tested concentrations had any effect on the release, meaning that no dose-dependent influence could be demonstrated. In contrast to that, a slight decrease of CXCL1 by high concentrations of Jak inhibitor I was observed in MALP-2-stimulated microglia. The two highest concentrations of $100 \mathrm{nM}$ and $500 \mathrm{nM}$ 
reduced the CXCL1 production by $20 \%$ and $35 \%$, respectively. This points towards a dose-dependent, but rather low effect of the inhibitor on MALP-2-triggered CXCL1 release.

A

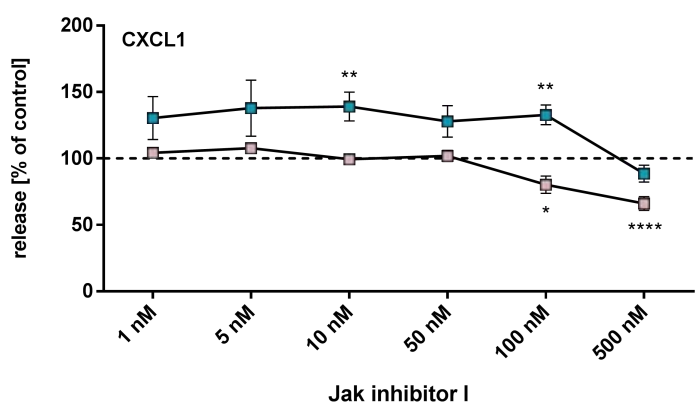

C

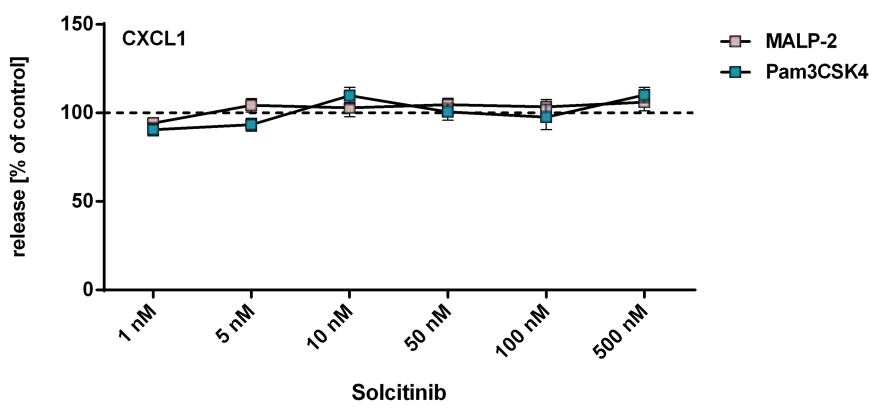

B

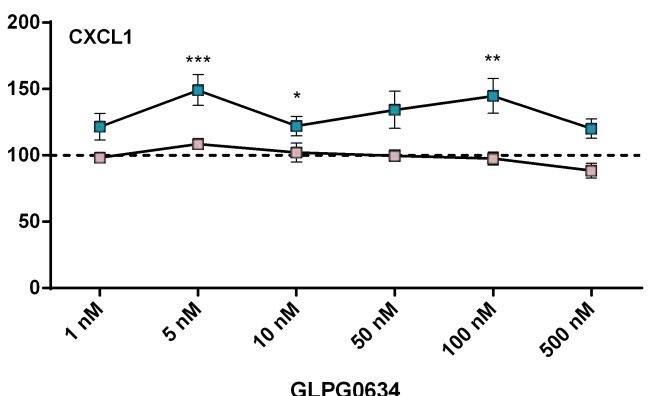

GLPG0634

Figure 4.34: Janus kinase inhibition does not affect CXCL1 and CCL2 production in response to TLR2 stimulation in a dose-dependent manner. Cultured microglia from wt mice were pre-incubated with the indicated doses of (A) Jak inhibitor I, (B) GLPG0634 or (C) Solcitinib for $1 \mathrm{~h}$ and afterwards stimulated with $10 \mathrm{ng} / \mathrm{mL}$ Pam3CSK4 or MALP-2 for $18 \mathrm{~h}$ in the presence of the respective dose of the inhibitor. CXCL1 release was determined in cell culture supernatants by ELISA. Absolute values were normalized to amounts produced by cells stimulated in the absence of the inhibitor. Data are mean \pm SEM with $n=12$ from 3 independent experiments. Statistical analysis compares the release in the presence of the respective inhibitor at the indicated concentration with the release in the absence of the inhibitor and was performed using the Mann-Whitney test, ${ }^{*}$ represents $p \leq 0.05,{ }^{* *}$ represents $p \leq 0.01,{ }^{* * *}$ represents $p \leq 0.001,{ }^{* * * *}$ represents $p \leq 0.0001$.

Because no direct link between overall janus kinase inhibition and CXCL1 production in response to Pam3CSK4 or MALP-2 could be established, the individual role of Jak1 and Tyk2 should be determined. For a more selective Jak1 inhibition, GLPG0634 was used, which has a markedly lower IC50 value for Jak1 than Tyk2. Increasing concentrations of this inhibitor were applied to Pam3CSK4- or MALP2-stimulated microglia in the same way as described above. Similar to Jak inhibitor I, GLPG0634 had an unexpected influence on Pam3CSK4-triggered CXCL1 levels. Concentrations of $5 \mathrm{nM}, 10 \mathrm{nM}$ and $100 \mathrm{nM}$, but no other concentration, significantly increased the CXCL1 production (Figure 4.34 B). However, there was no general trend observable, meaning that in principle the CXCL1 release remained more or less stable around the control level. This was even more obvious in response to MALP-2 stimulation, where no difference between inhibitor presence or absence was observed.

For verifying this low impact of Jak1 inhibition on the CXCL1 release, a selective Jak1 inhibitor, namely Solcitinib, was used. As shown in Figure 4.34 C, neither Pam3CSK4- nor MALP-2-stimulated microglia changed their CXCL1 production by the presence of this inhibitor. Irrespective of the used concentration, CXCL1 levels were always completely the same as in the absence of the inhibitor. So indeed, blocking the kinase activity of Jak1 did not result in alterations of CXCL1. 


\subsubsection{Tyk2 ${ }^{E 775 K}$ microglia show properties that deviate from the previous re- sults}

With the help of different kinase inhibitors and especially the Jak1-specific inhibitor Solcitinib, it was shown that Jak1 is not involved in the regulation of CXCL1 release. Furthermore, it was already partially indicated by overall janus kinase inhibition with Jak inhibitor I that Tyk2 does also not play a role in this scenario. For proving this, responses of tyk2 ${ }^{E 775 K}$ microglia to Pam3CSK4 and MALP-2 stimulation were evaluated in comparison to wt cells. The CXCL1 release is summarized in Figure 4.35 A. Surprisingly, the tyk2 missense mutation resulted in a substantial decrease of CXCL1 production by about $80 \%$, irrespective of the used TLR2 ligand.

A

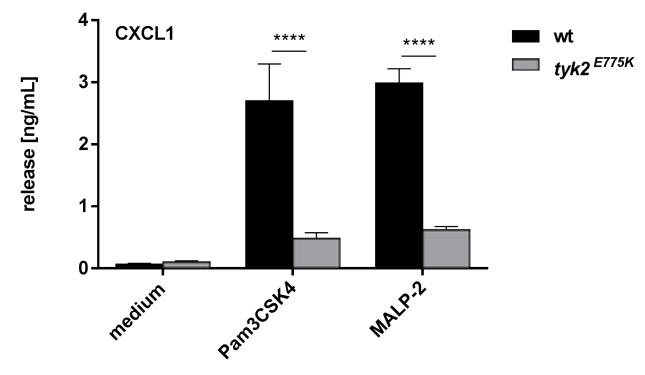

B

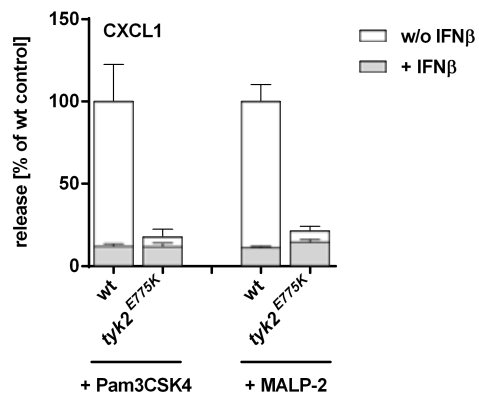

Figure 4.35: Tyk2 ${ }^{E 775 K}$ microglia produce less CXCL1 than wt cells in response to TLR2 stimulation and this production can be even lowered by IFN $\beta$ treatment. (A) Cultured microglia from wt and tyk2 ${ }^{E 775 K}$ mice were stimulated with $10 \mathrm{ng} / \mathrm{mL}$ Pam3CSK4 or MALP-2 for $18 \mathrm{~h}$. (B) Cells were stimulated as in (A) in the presence (+ IFN $\beta$ ) or absence (w/o IFN $\beta)$ of $10 \mathrm{ng} / \mathrm{mL}$ recombinant IFN $\beta$. CXCL1 release was determined in cell culture supernatants by ELISA. Data are mean \pm SEM with $n=16$ from 4 independent experiments. (A) Statistical analysis was performed using the Mann-Whitney test, ${ }^{* * * *}$ represents $p \leq 0.0001$. (B) Absolute values were normalized to amounts produced by wt cells stimulated in the absence of IFN $\beta$.

In order to find an explanation for this unexpected result, it was investigated whether maybe type I interferon signaling is still functional in the tyk $2^{E 775 K}$ microglia. As explained in chapter $4.2, \operatorname{IFN} \beta$ is able to negatively regulate CXCL1 production in response to TLR4 agonists. If tyk $2^{E 775 K}$ cells would be able to signal upon type I interferon treatment, IFN $\beta$ could possibly also decrease CXCL1 upon TLR2 stimulation. Therefore, high amounts of IFN $\beta$ were applied to wt and tyk2 ${ }^{E 775 K}$ microglia together with Pam3CSK4 or MALP-2 for induction of TLR2-dependent CXCL1 release. As expected, addition of IFN $\beta$ decreased the CXCL1 level in wt microglia by nearly $90 \%$ (Figure $4.35 \mathrm{~B}$ ). This is similar to what was observed for IFN $\beta$ treatment of TLR4-stimulated wt cells (see Figure 4.19, 4.23 and 4.25). Interestingly, tyk $2^{E 775 K}$ microglia did also respond to IFN $\beta$, meaning that the already really low CXCL1 production in these cells could be further decreased by about $30 \%$. This indicates that the tyk2 missense mutation does not disrupt the type I interferon signaling completely.

A possibility to entirely block type I interferon signaling in the $t y k 2^{E 775 K}$ microglia would be the inhibition of all janus kinases. This was achieved by using Jak inhibitor I in these cells. Because this inhibitor targets Jak1 and Tyk2, it should also block remaining kinase activity of Tyk2 in tyk2 ${ }^{E 775 K}$ microglia. A comparison of wt and tyk $2^{E 775 K}$ cells treated with increasing concentrations of Jak inhibitor I is shown in Figure 4.36 A and B. Similar to what was described in chapter 4.3.8, the inhibitor had no dose-dependent effect on the production of CXCL1 by wt microglia in response to Pam3CSK4 or MALP-2. Only some concentrations affected the CXCL1 level, but mostly, the release stayed around the control level. When analyzing the $t y k 2^{E 775 K}$ microglia, also no effect of treatment with Jak inhibitor I was observed. The CXCL1 production constantly stayed below $15 \%$ of the release that was obtained by wt cells, which were stimulated in the absence of the inhibitor. Even the highest concentration of $500 \mathrm{nM}$ could not 
increase this really low $C X C L 1$ level in tyk2 ${ }^{E 775 K}$ microglia. This indicates that these cells are in general impaired in their CXCL1 production.

In case of such a general impairment, also specific Jak1 inhibition should not change the CXCL1 release by tyk2 ${ }^{E 775 K}$ microglia. Thus, these cells were treated with Solcitinib, which only blocks Jak1, but not Tyk2 activity. Treatment with this inhibitor revealed a similar pattern as described for Jak inhibitor I. Solcitinib also had no dose-dependent effect on the production of CXCL1 by wt microglia in response to Pam3CSK4 or MALP-2 (Figure 4.36C and D). Even though some concentrations affected the CXCL1 levels, no overall trend was observable. In $t y k 2{ }^{E 775 K}$ microglia, the CXCL1 production was not affected at all by increasing concentrations of Solcitinib. The release stayed below $15 \%$ of the wt control and could not be changed by any inhibitor concentration. This supports the assumption that tyk2 ${ }^{E 775 K}$ microglia are generally impaired in producing CXCL1.

A

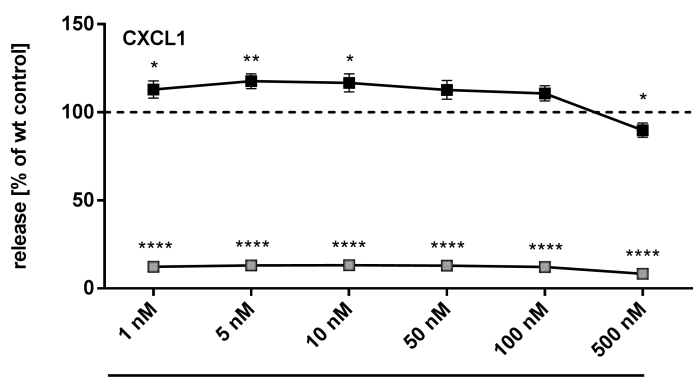

Jak inhibitor I+ Pam3CSK4

C

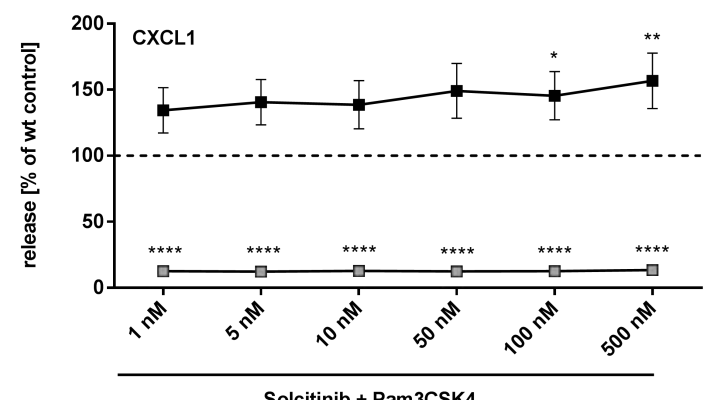

B

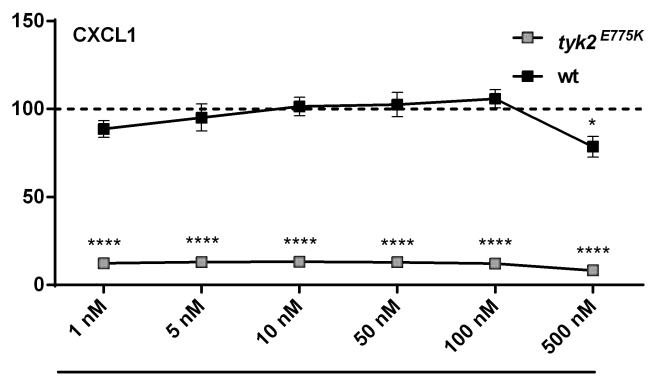

Jak inhibitor I+ MALP-2

D

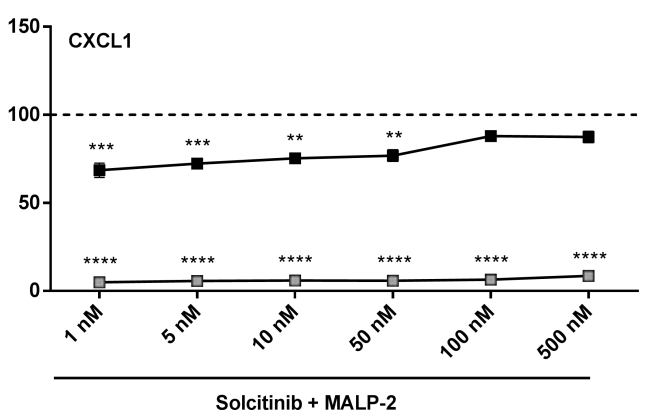

Figure 4.36: Janus kinase inhibition does not affect TLR2-induced CXCL1 production by tyk2 ${ }^{E 775 K}$ microglia. Cultured microglia from wt and tyk2 ${ }^{E 775 K}$ mice were pre-incubated with the indicated doses of (A), (B) Jak inhibitor I or (B), (C) Solcitinib for $1 \mathrm{~h}$ and afterwards stimulated with $10 \mathrm{ng} / \mathrm{mL}$ (A), (C) Pam3CSK4 or (B), (D) MALP-2 for $18 \mathrm{~h}$ in the presence of the respective dose of the inhibitor. CXCL1 release was determined in cell culture supernatants by ELISA. Absolute values were normalized to amounts produced by wt cells stimulated in the absence of the inhibitor. Data are mean \pm SEM with $n=16$ from 4 independent experiments. Statistical analysis compares the release of both genotypes stimulated in the presence of the inhibitor to the release of wt cells stimulated in the absence of the inhibitor and was performed using the Mann-Whitney test, * represents $p \leq 0.05$, ${ }^{* *}$ represents $p \leq 0.01,{ }^{* * *}$ represents $p \leq 0.001,{ }^{* * * *}$ represents $p \leq 0.0001$.

\subsubsection{Regulation of CXCL1 production is independent of STAT1 and STAT2}

STAT1 and STAT2 are the most important downstream effectors of the canonical type I interferon signaling pathway. They shape type I interferon responses by regulating the expression of ISGs upon activation of IFNAR (Platanias 2005). Because IFNAR1 and IFNAR2 were shown to cooperatively affect the production of CXCL1, the two STAT proteins could be important for mediating this effect.

This would require that STAT1 and STAT2 are activated in response to TLR2 challenges. Such an activation would be accompanied by elevated expression of both transcription factors. Thus, microglia were stimulated with Pam3CSK4 or MALP-2 for $3 \mathrm{~h}$ and the STAT1 and STAT2 expression was determined by 
A

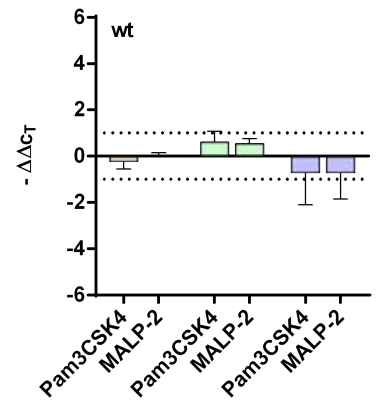

B

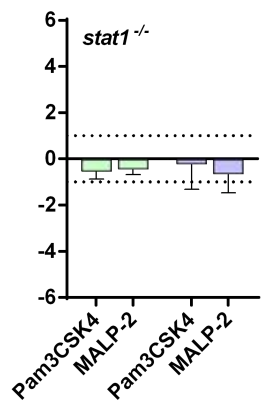

C

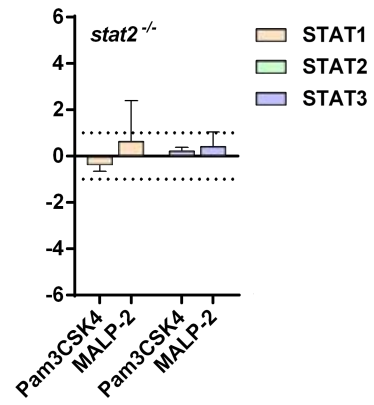

Figure 4.37: Expression of STAT1, STAT2 and STAT3 is not changed by TLR2 stimulation in wt, stat1\% and stat2 $\%$ microglia. Cultured microglia from (A) wt, (B) stat $1^{-/}$and (C) stat2 ${ }^{-/}$mice were stimulated with $10 \mathrm{ng} / \mathrm{mL}$ Pam3CSK4 or MALP2 for $3 \mathrm{~h}$. RNA was isolated by using the RNeasy Mini Kit and converted into cDNA by using the QuantiTect ${ }^{\circledR}$ Reverse Transcription Kit. Real-Time PCR was performed by using the iTaq ${ }^{\mathrm{TM}}$ Universal SYBR ${ }^{\circledR}$ Green Supermix. Gapdh served as internal control and unstimulated cells were regarded as baseline gene expression control. The interval between the dotted lines indicates no change in gene expression. Data are mean $\pm S E M$ with $n=3$ from 3 independent experiments.

RT-PCR. As a comparison, STAT3 was also analyzed. According to the explanation in chapter 4.2.13, $-\Delta \Delta c_{T}$ values were calculated and served as relative measurement for up- or down-regulation of the respective genes. Values greater than 1 represent an up-regulation and values less than -1 a downregulation. As shown in Figure $4.37 \mathrm{~A}$, all $-\Delta \Delta \mathrm{c}_{\mathrm{T}}$ values were within the 1 to -1 interval. Hence, the expression of none of the three STAT proteins was changed by activation of TLR2.

However, the expression of STAT genes could be different in the absence of either of these transcription factors. Lack of one STAT protein could result in an increase of another STAT. In order to test this hypothesis, stat $\%$ and stat $\%$ microglia were analyzed. Both genotypes were stimulated with Pam3CSK4 or MALP-2 and the STAT1, STAT2 and STAT3 expression was measured. Similar to what was observed in wt cells, no change in gene expression upon TLR2 stimulation could be demonstrated. STAT1-deficient microglia did not up-regulate STAT2 or STAT3 (Figure 4.37B) and STAT2-deficient microglia showed no increase in STAT1 or STAT3 levels (Figure 4.37 C). This is in line with the above described phenomenon that STAT1, STAT2 or STAT3 are not activated by TLR2 agonists in wt microglia.

A

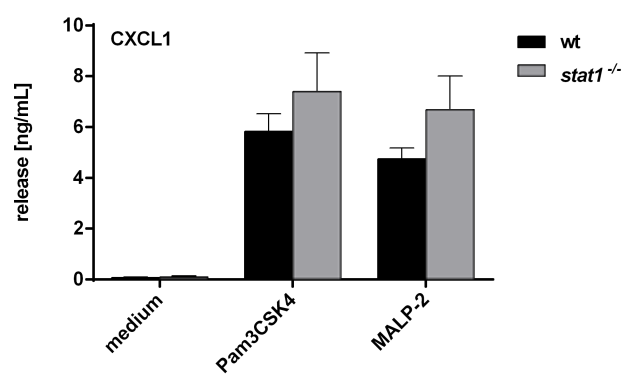

B

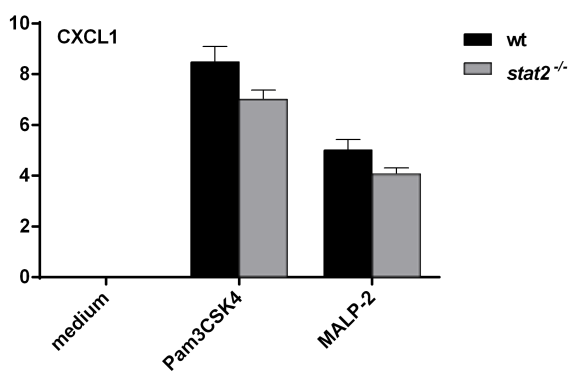

Figure 4.38: STAT1 and STAT2 deficiency do not affect CXCL1 production by TLR2-stimulated microglia. Cultured microglia from wt and (A) stat $1^{\%}$ or (B) stat $2^{--}$mice were stimulated with $10 \mathrm{ng} / \mathrm{mL}$ Pam3CSK4 or MALP-2 for $18 \mathrm{~h}$. CXCL1 release was determined in cell culture supernatants by ELISA. Data are mean \pm SEM with $(\mathbf{A}) \mathrm{n}=16$ from 4 independent experiments and (B) $n=24$ from 6 independent experiments. Statistical analysis was performed using the Mann-Whitney test.

However, this does not necessarily mean that STAT1 and STAT2 could not be involved in IFNARmediated regulation of CXCL1 production. For directly demonstrating a contribution of the two transcription factors to the effect of IFNAR, stat $\%$ and stat2 $\%$ microglia were used. Both genotypes were stimulated with Pam3CSK4 or MALP-2 and the release of CXCL1 was quantified. If STAT1 and STAT2 would mediate the regulation of this chemokine, an overproduction as observed in the absence of IF- 
NAR1 (see Figure 4.27 B) or IFNAR2 (see Figure 4.32) would be expected. However, no difference in the production of CXCL1 was observed in the absence of either STAT1 or STAT2 in comparison to wt levels irrespective of the used TLR2 ligand (Figure $4.38 \mathrm{~A}$ and B). So indeed, both transcription factors did not participate in the regulation of CXCL1 as it was indicated by the expression analysis.

\subsection{Type I interferon signaling controls TLR3-induced chemokine production}

The regulation of chemokine production in response to TLR4 activation was shown to be controlled by the canonical type I interferon signaling pathway. In contrast to this, only IFNAR was demonstrated to be involved in regulating TLR2-induced chemokines. So finally, a third TLR system was analyzed regarding the requirement of type I interferon signaling for control of chemokine production. With TLR4 and TLR2, two TLRs with differences in their signaling were chosen. While TLR4 uses the MyD88- and the TRIF-dependent signaling pathway, TLR2 recruits only MyD88 (Akira et al. 2006). To complete the picture, a TLR that requires only TRIF for its signaling was missing and thus, TLR3 was selected.

A

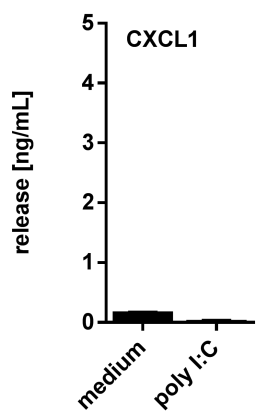

B

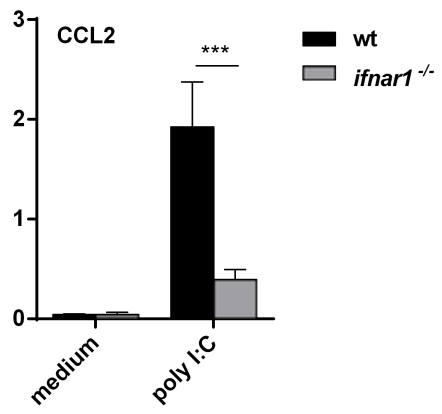

Figure 4.39: While CXCL1 is in general not released in response to TLR3 stimulation, IFNAR1 deficiency causes decreased CCL2 production by TLR3-stimulated microglia. Cultured microglia from (A), (B) wt and (B) ifnar1\% mice were stimulated with $50 \mu \mathrm{g} / \mathrm{mL}$ poly I:C for $18 \mathrm{~h}$. (A) CXCL1 and (B) CCL2 release were determined in cell culture supernatants by ELISA. Data are mean \pm SEM with $n=12$ from 3 independent experiments. Statistical analysis was performed using the MannWhitney test, ${ }^{* * *}$ represents $p \leq 0.001$.

However, in this system just a reduced set of experiments was performed, which focused on the most important parts of the canonical type I interferon signaling pathway. Because CXCL1 was not produced in response to TLR3 stimulation (Figure 4.39 A), the analysis of chemokines was limited to CCL2.

First of all, the involvement of IFNAR in the regulation of CCL2 release in response to poly I:C as representative TLR3 agonist was assessed. In order to model receptor deficiency, IFNAR1-deficient microglia were used. These cells were stimulated with poly I:C and the production of CCL2 was measured in comparison to wt levels. In the absence of IFNAR1, CCL2 release was significantly decreased as ifnar $1 \%$ microglia produced $80 \%$ less CCL2 than wt cells (Figure $4.39 \mathrm{~A}$ ). This shows that the regulation of TLR3-triggered CCL2 production depends on IFNAR1.

A similar phenomenon was also observed in other tissue macrophages. In the absence of IFNAR1, BMDM failed to produce any CCL2 in response to poly I:C (Figure 4.40). Thus, the effect of IFNAR1 deficiency on CCL2 release was even more striking than in microglia. In contrast to this, $\mathrm{pM} \Phi$ showed the same production of CCL2 in the presence or absence of IFNAR1. However, this production was in general very low compared to the CCL2 levels that were obtained by microglia or BMDM.

Downstream of IFNAR, the two transcriptions factors STAT1 and STAT2 were analyzed in terms of their contribution to the IFNAR1-mediated regulation of CCL2. By using stat $1^{\%}$ and stat2 ${ }^{-1}$ microglia, it 

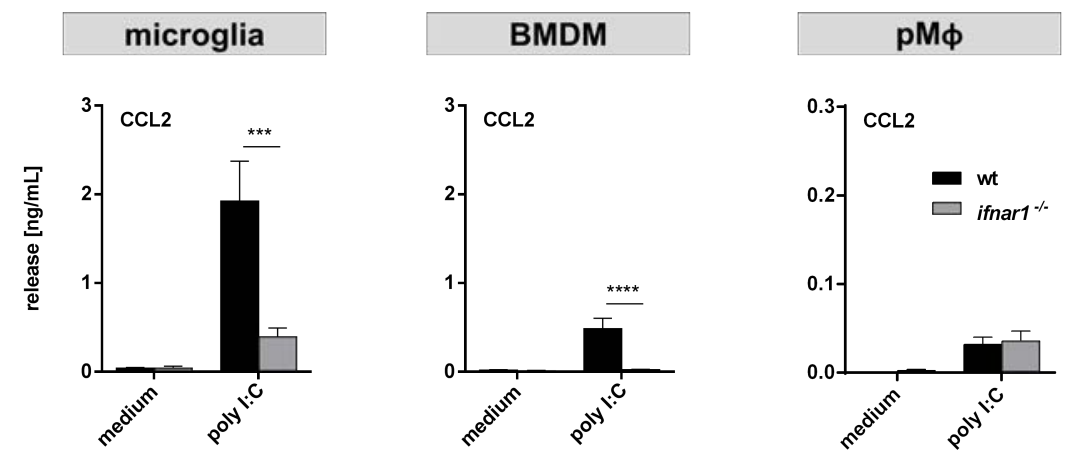

Figure 4.40: IFNAR1 deficiency similarly affects TLR3-induced CCL2 production by microglia and BMDM, but not by $\mathrm{pM} \Phi$. Cultured microglia, BMDM and $\mathrm{pM} \Phi$ from wt and ifnar $1 \%$ mice were stimulated with $50 \mu \mathrm{g} / \mathrm{mL}$ poly $\mathrm{l}: \mathrm{C}$ for $18 \mathrm{~h}$. CCL2 release was determined in cell culture supernatants by ELISA. Data are mean \pm SEM with $n=8-12$ from 3-4 independent experiments. Statistical analysis was performed using the Mann-Whitney test, ${ }^{* * *}$ represents $p \leq 0.001,{ }^{* * * *}$ represents $p \leq 0.0001$.

A

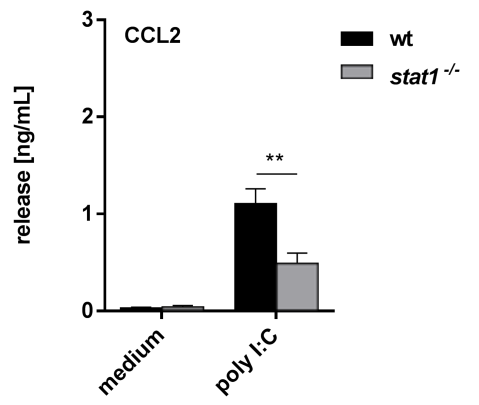

B

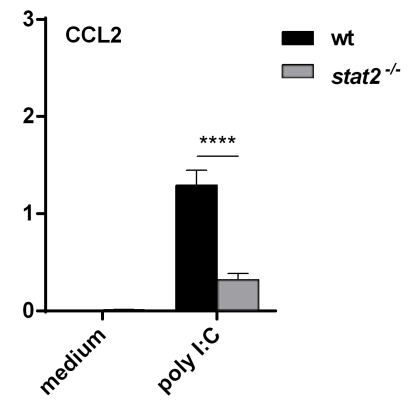

Figure 4.41: STAT1 and STAT2 deficiency cause decreased CCL2 production by TLR3-stimulated microglia. Cultured microglia from wt and (A) stat $1 \%$ or (B) stat ${ }^{\%}$ mice were stimulated with $50 \mu \mathrm{g} / \mathrm{mL}$ poly I:C for $18 \mathrm{~h}$. CCL2 release was determined in cell culture supernatants by ELISA. Data are mean \pm SEM with $(A) n=16$ from 4 independent experiments and (B) $n=24$ from 6 independent experiments. Statistical analysis was performed using the Mann-Whitney test, ${ }^{* *}$ represents $p \leq 0.01,{ }^{* * * *}$ represents $\mathrm{p} \leq 0.0001$.

could be demonstrated that adequate CCL2 production depends on the presence of both transcriptions factors. In the absence of STAT1, CCL2 levels were reduced by $50 \%$, while in STAT2-deficient cells the reduction was even more than $75 \%$ (Figure $4.41 \mathrm{~A}$ and B). Thus, STAT1 and STAT2 seem to be mediators of the IFNAR1-dependent control of TLR3-induced CCL2 production.

A

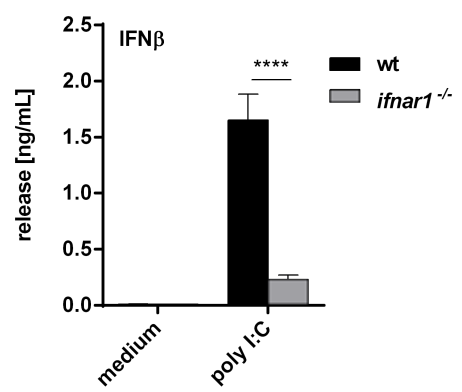

B

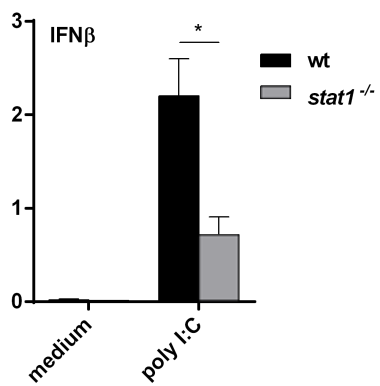

C

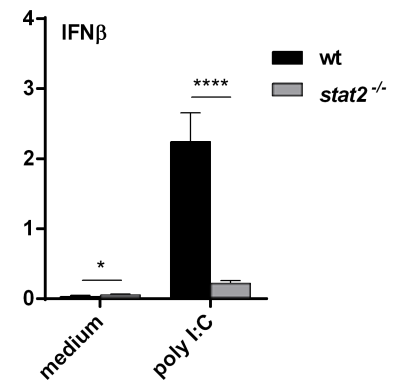

Figure 4.42: Deficient type I interferon signaling impairs IFN $\beta$ production by TLR3-stimulated microglia. Cultured microglia from wt and (A) ifnar $1^{\%}$, (B) stat $1^{\%}$ or (C) stat2 $\%$ mice were stimulated with $50 \mu \mathrm{g} / \mathrm{mL}$ poly I:C for $18 \mathrm{~h}$. IFN $\beta$ release was determined in cell culture supernatants by ELISA. Data are mean \pm SEM with (A) $n=12$ from 3 independent experiments, (B) $n=16$ from 4 independent experiments and $(C) n=24$ from 6 independent experiments. Statistical analysis was performed using the Mann-Whitney test, * represents $p \leq 0.05$, ${ }^{* \star *}$ represents $p \leq 0.0001$. 
However, deficient type I interferon signaling did not only affect the release of CCL2, but also impaired IFN $\beta$ production. In ifnar $1 \%$, stat $1 \%$ and stat $\%$ microglia, IFN $\beta$ levels were significantly reduced compared to wt. This reduction ranged from $65 \%$ in ifnar $1^{\%}$ and stat $1 \%$ microglia to $90 \%$ in stat ${ }^{\%}$ cells (Figure $4.42 \mathrm{~A}, \mathrm{~B}$ and $\mathrm{C}$ ). These results indicate that functional type I interferon signaling is required for ensuring the high levels of IFN $\beta$ that are produced in response to TLR3 activation. 


\section{Discussion}

The CNS is an essential organ for survival and thus, definitely needs to be defended from harmful threats. As first line of defense, the innate immune system is crucial for protection against pathogens or tissue damage. Within the CNS, microglia serve as principle innate immune cells that can initiate a variety of responses and execute different protective functions depending on the respective context. In this regard, they are able to secrete various soluble factors that further shape the immune reaction. Among these, interferons are of great importance for fighting viral and also bacterial infections. Apart from that, they have been implicated in several autoimmune diseases and even homeostatic processes (Owens et al. 2014). During the last years, increasing evidence for additional immunomodulatory functions of interferons accumulated. It has been shown that they regulate innate and adaptive immune responses under physiological and pathological conditions. On the one hand, they restrain the development of several immunopathologies and are even approved as treatment and on the other hand, they can increase the severity of some diseases (González-Navajas et al. 2012). Interestingly, the molecular mechanisms by which interferons exert these opposing regulatory functions are still largely unknown. Nevertheless, especially negative control of immune responses could be clearly protective for the host, considering that excessive inflammatory reactions can lead to tissue impairment and even death. Since the CNS has a restricted cell renewal and regenerative capacity, it is extremely vulnerable to uncontrolled autodestructive immune and inflammatory responses, which makes a tight control of these processes even more important (Gao and Hong 2008). Therefore, we investigated how microglial responses to CNS infection and damage are regulated by IFN $\beta$ as representative interferon and propose a mechanism how it exerts its effects.

\subsection{Microglia mainly produce IFN $\beta$ in response to activation of different TLRs}

Interferons are signaling molecules within the immune system that can be released upon encounter with several pathogens, such as bacteria, viruses and parasites. They regulate immune responses and serve important functions in fighting infections. Their production is induced by a large variety of PRRs, including TLRs (Trinchieri 2010). TLRs are expressed on cells of the innate immunity in order to detect pathogens and tissue damage by recognition of PAMPs and DAMPs, respectively. Within the CNS, most of the different TLRs are expressed on microglia as the tissue-resident macrophage population (Kettenmann et al. 2011). Thus, these cells are in principle capable of producing interferons. About 12 interferon species have been identified that are classified in three different types (Pestka 2007). The subgroup of type I interferons includes several interferon proteins with IFN $\alpha$ and IFN $\beta$ being the immunologically most relevant. Therefore, the production of these two molecules by microglia was assessed in response to activation of different TLRs. 
Interferons have important antiviral functions, meaning that they are produced during viral infections (Sen 2001). A molecular pattern associated with such infections is double-stranded RNA, which can be recognized by TLR3 (Akira et al. 2006). As a synthetic analog of double-stranded RNA, poly I:C also activates TLR3 (Takeuchi and Akira 2010). In line with previous studies in human embryonic kidney cells (Alexopoulou et al. 2001), we showed that IFN $\beta$ expression was induced by poly I:C in microglia. Even though only moderate expression levels were detected, these resulted in massive amounts of protein production. While no expression of four different IFN $\alpha$ subtypes (IFN $\alpha B, 6,12,14$ ) was detected, substantial IFN $\alpha$ release in response to poly I:C could be measured. Thus, microglia seem to fight viral infections by producing both type I interferons. Indeed, they were shown to be, together with astrocytes, the major source of IFN $\beta$ in La Crosse virus-infected mice (Kallfass et al. 2012) and also produce IFN $\alpha$ in response to Theiler's murine encephalomyelitis virus (Olson and Miller 2004).

Apart from their involvement in viral infections, interferons also participate in immune responses to bacterial pathogens (Decker et al. 2005). Bacteria can be sensed by different TLRs, including TLR2 and TLR4. While the TLR2 complex mainly detects gram-positive bacteria, TLR4 is responsible for recognition of gram-negative bacteria (Kawai and Akira 2010). TLR2 is activated by different ligands that vary depending on the respective receptor heterodimer. For example, TLR1/2 recognizes the triacylated lipopeptide Pam3CSK4 (Jin et al. 2007) and TLR6/2 the diacylated lipopeptide MALP-2 (Takeuchi et al. 2001). In general, it is assumed that type I interferons are not produced in response to these bacterial TLR2 agonists (Toshchakov et al. 2002; Barbalat et al. 2009). Even though Dietrich et al. (2010) could show a low IFN $\beta$ production following stimulation with Pam3CSK4 or MALP-2, this seems to be highly dependent on the cell type and respective context. In our experiments, neither IFN $\alpha$ nor IFN $\beta$ was produced in response to Pam3CSK4 or MALP-2 as verified by mRNA expression analysis via RT-PCR and protein measurements by ELISA. Similarly, activation of TLR4 by Re-LPS failed to induce IFN $\alpha$ on mRNA and protein level, probably due to the lack of IRF7 induction (Noppert et al. 2007), which is required for IFN $\alpha$ production (Kawai et al. 2004). In contrast to this, several TLR4 agonists were able to induce IFN $\beta$ in microglia as it was already demonstrated by others (Jung et al. 2005; Regen et al. 2011; Menzfeld et al. 2015). According to these studies, a comparison of Re-LPS- and Pam3CSK4-induced type I interferon expression confirmed that only the TLR4 and not the TLR2 agonist triggers IFN $\beta$. Apart from Re-LPS, also S-LPS and live E. coli led to IFN $\beta$ release by microglia at an intermediate level. In line with previous studies, the production stayed markedly lower than the amount obtained by poly I:C stimulation (Jack et al. 2005). Nevertheless, it could be increased dependent on the dose of stimulus. The same holds true for FN, which dose-dependently induced IFN $\beta$ release similar to LPS and E. coli. FN represents a major component of the extracellular matrix, but is also an abundant soluble constituent of plasma (Pankov and Yamada 2002). Thus, it can serve as a DAMP indicating tissue damage or vascular leakage. Because IFN $\beta$ release was triggered by $\mathrm{FN}$, this points towards a role of IFN $\beta$ also in tissue impairment. Interestingly, FN-stimulated microglia had a much higher IFN $\beta$ mRNA expression than Re-LPS-stimulated cells, but a similar protein production. Accordingly, IFN $\beta$ release cannot be only transcriptionally controlled. Rather translational regulation needs to also occur as it was already demonstrated by others (Grafi et al. 1993).

The differences in induction of type I interferons between the examined TLR systems correlated with differences in their respective signaling pathways. In general, TLR signaling is initiated by TIR domaincontaining adaptor proteins (O'Neill and Bowie 2007). There exist two major signaling routes. One pathway utilizes MyD88, while the other recruits TRIF. Upon recruitment of TRIF, the activation of IRF3 leads to type I interferon production (Fitzgerald et al. 2003). In contrast to that, MyD88-dependent type I interferon production by endosomal TLRs and TLR2 requires IRF7 (Takeuchi and Akira 2010; Dietrich et al. 2010). Considering the TLR systems that we investigated, they differentially use the described 
signaling pathways. TLR3 exclusively signals via TRIF, TLR2 uses only MyD88 and TLR4 activates both signaling cascades (Akira et al. 2006). As only TLR3 and TLR4, but not TLR2 stimulation led to type I interferon production by microglia, we hypothesized that this might be due to the use of TRIF. Because IFN $\alpha$ was only released upon poly I:C treatment, we hereinafter focused on IFN $\beta$. Analysis of MyD88- and TRIF-deficient microglia revealed that indeed only TRIF is required for IFN $\beta$ production by microglia. While IFN $\beta$ levels were completely the same in wt and myd88\% cells, TRIF deficiency dramatically impaired IFN $\beta$ release. In response to TLR4 agonists, no IFN $\beta$ could be detected in trif ${ }^{\text {Ips } 2}$ microglia. Additionally, the production was decreased by one third in poly I:C-stimulated trif ${ }^{1 / p 2}$ cells. So similar to other studies (Yamamoto et al. 2003), we could show a TRIF dependence of microglial IFN $\beta$ production.

The TRIF-dependent signaling pathway is not initiated from the cell surfaces, but from endosomal compartments. TLR3 is located at endosomes and can therefore directly recruit the adaptor protein TRIF. However, TLR4 is a cell surface receptor and thus, the receptor complex needs to be internalized by endocytosis in order to get access to the TRIF signaling route (Kagan et al. 2008). This process is controlled by the TLR4 co-receptor CD14 (Zanoni et al. 2011). Because TRIF was shown to enable microglial IFN $\beta$ production, this link from CD14 to the TRIF-dependent signaling suggests a crucial role for CD14. Therefore, TLR4-stimulated microglia from wt and $c d 14 \%$ mice were compared concerning their IFN $\beta$ release. In CD14-deficient microglia, IFN $\beta$ production in response to Re-LPS was completely absent. This is in line with previous studies, showing that $c d 14^{-}$peritoneal macrophages, BMDM and dendritic cells do not produce IFN $\beta$ upon LPS stimulation (Jiang et al. 2005; Zanoni et al. 2011). Furthermore, also live E. coli and FN did not trigger IFN $\beta$ release by $c d 14^{-/}$microglia. This indicates a global dependence of different TLR4 agonists on the presence of CD14 for mounting IFN $\beta$ responses.

Overall, we demonstrated that microglia produce IFN $\alpha$ and IFN $\beta$ during viral infections as mimicked by poly I:C stimulation. Furthermore, TLR4 agonists related to gram-negative bacteria and tissue damage induced IFN $\beta$ release. This production depended on the TRIF signaling pathway, which is controlled by CD14 in TLR4-triggered responses. In contrast to that, bacterial ligands activating TLR2 failed to induce IFN $\alpha$ and IFN $\beta$ release by microglia.

\subsection{IFNAR controls chemokine production in response to TLR4, TLR2 and TLR3}

Apart from their important functions in fighting viral and bacterial infections, interferons exert a variety of immunomodulatory functions. They regulate innate as well as adaptive immune responses under physiological and pathological conditions. This includes modulation of generation, trafficking and effector activity of several immune cells. In this regard, interferons can control cell trafficking by regulating the expression of different chemokines in response to various stimuli or diseases (Rauch et al. 2013). The activation of TLRs by stimulation with PAMPs and DAMPs leads to the production of chemokines as well as type I interferons. This means that interferons could regulate TLR-induced chemokines within the same response. As shown by Regen et al. (2011), IFN $\beta$ differently regulates several chemokines that were released by microglia in response to different variants of the TLR4 agonist LPS. Among these chemokines, CXCL1 was clearly less produced in the presence of IFN $\beta$. A similar effect was observed for TLR2-induced responses, where CXCL1 levels were also decreased by IFN $\beta$ treatment (Regen, unpublished). The regulation of TLR4-induced CXCL1 was further proven to be CD14-dependent (Janova et al. 2016). In the absence of CD14, microglia overreacted to strong infectious stimuli and this also manifested in a massive overproduction of CXCL1. However, normal CXCL1 levels could be restored by 
an IFN $\beta$-mediated feedback mechanism. In contrast to that, other chemokines showed opposing features. As an example, LPS-induced CCL2 production was not increased, but even slightly decreased in $\mathrm{cd}_{14}{ }^{-/}$microglia. However, normal CCL2 levels could be regained by addition of IFN $\beta$. Because IFN $\beta$ was shown to opposingly regulate $\mathrm{CXCL} 1$ and $\mathrm{CCL} 2$, the mechanism underlying this effect was investigated. In response to all type I interferons, the canonical type I interferon signaling pathway is initiated. Thus, this pathway could be essential for controlling TLR-induced chemokine production.

The cell surface receptor that recognizes all type I interferons is called IFNAR. It consist of the two subunits IFNAR1 and IFNAR2 that are both required for effective ligand binding (Cohen et al. 1995). Consequently, this receptor would be the first critical step of an IFN $\beta$-mediated regulatory mechanism. In order to dissect the role of IFNAR in this setting, receptor deficiency was modeled by IFNAR1 knockout (Müller et al. 1994). Microglia from ifnar1 ${ }^{\%}$ mice were isolated and their chemokine production in response to the stimulation of different TLRs was analyzed. Similar to the investigation of type I interferon production, experiments focused on TLR4, TLR2 and TLR3 as representative TLR systems. According to their potential to be regulated by IFN $\beta$, chemokine measurements included CXCL1 and CCL2. However, only TLR4 agonists were able to induce both chemokines. In contrast to this, we could not detect any CCL2 $18 \mathrm{~h}$ after Pam3CSK4 or MALP-2 stimulation. This can be explained by the fact that CCL2 production in response to TLR2 agonists needs more than $18 \mathrm{~h}$ to be established as indicated by previous work (Regen, unpublished) showing that no plateau in microglial CCL2 levels is present even $72 \mathrm{~h}$ after Pam3CSK4 or MALP-2 stimulation. Apart from that, no CXCL1 was released by poly I:C-stimulated microglia.

When comparing ifnar1 ${ }^{\%}$ with wt microglia, dramatic changes in their CXCL1 and CCL2 release were observed. CXCL1 production was increased in response to all tested TLR4 and TLR2 agonists, but the overproduction was markedly higher in TLR4- than TLR2-stimulated cells. Nevertheless, this effect was shown to be present over a time period of $48 \mathrm{~h}$ in both TLR systems, indicating a stable effect that cannot be rescued by time. So similar to CD14 deficiency (Janova et al. 2016), IFNAR1 knock-out resulted in a loss of negative regulation of microglial CXCL1 production in response to TLR4 stimulation. This directly links the CD14-dependent IFN $\beta$-mediated feedback to type I interferon signaling. However, this mechanism cannot account for the TLR2 system. Even though CD14 was demonstrated to enhance TLR2 activity (Yoshimura et al. 1999), no increase of CXCL1 production in the absence of CD14 could be shown (Janova, unpublished). Furthermore, as described in chapter 4.1, no IFN $\beta$ was released upon Pam3CSK4 or MALP-2 stimulation. Thus, overproduction of CXCL1 in response to TLR2 stimulation can neither be attributed to CD14 nor to an IFN $\beta$-mediated regulatory feedback. Interestingly, the regulation of CXCL1 by IFNAR1 seems to be highly dependent on the respective context. CXCL1 production was shown to be higher in influenza-infected ifnar $1 \%$ mice following secondary infection with S. pneumoniae as compared to wt controls (Shahangian et al. 2009). Since S. pneumoniae activate TLR2 (Yoshimura et al. 1999; Mogensen et al. 2006), this is in line with our data concerning overproduction of CXCL1 in TLR2-challenged ifnar $1^{-/}$microglia. In contrast to that, early CXCL1 expression was diminished in kidneys of Candida albicans-infected ifnar $1^{\%}$ compared to wt mice (Majer et al. 2012). A possible explanation could be that sensing of Candida albicans requires cooperative recognition by CLRs and TLRs (Netea et al. 2006), while we only investigated responses to TLRs. This would argue for a differential regulatory impact of IFNAR on chemokine production depending on the type of the triggering receptor. Additionally, this could also indicate a cell type-specificity, since we observed an effect of IFNAR1-deficiency on microglial CXLC1 production and not on kidney cells.

Regarding CCL2 production, TLR4- and TLR3-stimulated microglia were similarly affected by IFNAR1 deficiency. Under both conditions, ifnar $1^{-}$microglia released less CCL2 than wt cells. Similar to what 
was described for $\mathrm{CXCL1}$, these results indicate that IFN $\beta$ regulates microglial chemokine production via its receptor IFNAR. This is supported by the fact that CCL2 production was shown to be controlled by IFNAR during the late phase of Listeria monocytogenes infection (Jia et al. 2009) and septic peritonitis (Weighardt et al. 2006). Since IFN $\beta$ was released in response to LPS and poly I:C, its regulatory effect applies to the TLR4 as well as the TLR3 system. In both cases, IFN $\beta$-mediated regulation could be attributed to CD14, as this is not only used as TLR4 co-receptor (Wright et al. 1990), but also enhances TLR3 activation in response to poly I:C (Lee et al. 2006).

However, these results do not necessarily refer to the absence of IFNAR1. If ifnar $1^{\%}$ microglia would have an impairment in IFN $\beta$ production, this would cause a similar outcome. As shown for $\mathrm{cd} 14^{-/} \mathrm{mi}-$ croglia, the lack of IFN $\beta$ leads to a dysregulation of several chemokines, including CXCL1 and partially CCL2 (Janova et al. 2016). Thus, alterations of IFN $\beta$ levels in ifnar $1^{-/}$microglia had to be ruled out. In response to several TLR4 agonists, no difference between IFN $\beta$ release by wt and ifnar ${ }^{\%}$ cells was observed. So indeed, the IFNAR1 deficiency rather than an impairment in IFN $\beta$ seems to be causative for the observed alterations in the production of CXCL1 and CCL2. However, this was different for poly I:C-triggered responses. In this case, IFN $\beta$ levels were dramatically reduced in the absence of IFNAR1. Most likely, this is due to lack of the positive feedback that normally amplifies IFN $\beta$ production during viral infections. This amplification is initiated by small amounts of IFN $\beta$ that bind to IFNAR and thereby lead to the release of IFN $\alpha$ and more IFN $\beta$ (Sato et al. 1998). As a consequence, IFN $\beta$ production cannot be enhanced in ifnar $1^{\%}$ cells. Accordingly, we could not conclude whether IFNAR1 deficiency or this lowered IFN $\beta$ level causes the decreased CCL2 production in poly I:C-stimulated ifnar ${ }^{\%}$ microglia.

Microglia are tissue-resident macrophages of the CNS, but within different other tissues there exists a variety of these tissue-resident cells. This includes, for example, peritoneal macrophages in serosal tissue or bone marrow macrophages within the bone (Davies et al. 2013). These cells differ from microglia not only in their location, but also in their origin (Epelman et al. 2014). Nevertheless, they share many functions in terms of tissue surveillance and responses to infection. For this reason, the regulation of their chemokine production could be similarly organized. In order to test this, microglia, $\mathrm{pM} \Phi$ and BMDM from wt and ifnar $1 \%$ mice were compared regarding their CXCL1 and CCL2 release in response to TLR stimulation. When stimulated with agonists for TLR4, TLR2 and TLR3, microglia and BMDM showed overall a similar release pattern. CXCL1 was increased and CCL2 decreased in the absence of IFNAR1. In contrast to that, $\mathrm{pM} \Phi$ reacted partially different. While they showed the same picture as microglia and BMDM in response to TLR4 activation, IFNAR1 deficiency did not change their TLR2-induced CXCL1 and TLR3-induced CCL2 levels. A possible explanation could lie in the different location of these cells. Microglia reside in tissue of an immune-privileged organ (Galea et al. 2007) and the bone marrow is also mainly separated from the rest of the body providing immune-privileged sites for haematopoietic stem cells (Mercier et al. 2012). In contrast to that, $\mathrm{pM} \Phi$ are located within the peritoneal cavity, which is in close proximity to the gastro-intestinal tract. Thus, $\mathrm{pM} \Phi$ come into contact with bacterial and viral products from either invading pathogens or the microbiota more frequently than microglia or BMDM. This could require additional regulatory mechanisms that prevent excessive activation of $\mathrm{pM} \Phi$, so that IFNAR1 only partially controls the chemokine production of these cells.

However, using a complete knock-out system bears some risks as it affects all cells within the body also already during development. So far, all described results concerning effects of IFNAR1 deficiency were obtained by using ifnar $1 \%$ cells isolated from mice with a global knock-out of IFNAR1. Because IFNAR1 is expressed in all tissues and organs, this could have far-reaching functional consequences (de Weerd et al. 2007). Ifnar ${ }^{1 /}$ mice are more susceptible to viral infections (Müller et al. 1994), have impaired responses to parasites (Xin et al. 2010) and show enhanced osteoclastogenesis with decreased bone 
density (Teitelbaum and Ross 2003). These systemic alterations suggest that also ifnar $1^{\text {- }}$ microglia could have impairments in their immune functions. In order to rule out knock-out specific, intrinsic problems, IFNAR1 was functionally blocked in wt cells. This system has the advantage of properly developed, fully functional cells that only cannot activate IFNAR1. Thus, differences in their chemokine production can be completely attributed to the presence or absence of the IFNAR1-blocking antibody. Indeed, IFNAR1 blockade revealed very similar results to IFNAR1 knock-out. In response to TLR4 and partially TLR2 stimulation, an increase in CXCL1 and decrease in CCL2 levels was observed. Only CXCL1 production by Pam3CSK4-stimulated microglia was not enhanced by the IFNAR1-blocking antibody, which might be due to incomplete receptor blockade. So overall, IFNAR1 blockade could mimic the properties of the knock-out. However, effects in IFNAR1-blocked microglia were not as striking as in ifnar $1^{-}$cells. Most probably, this is due to a reduced efficiency of the blocking antibody compared to a $100 \%$ effective knock-out. Nevertheless, this proves that the chemokine production by ifnar $1^{-1}$ microglia is not altered per se, but rather IFNAR1 directly regulates CXCL1 and CCL2 production.

Even though IFNAR1 and IFNAR2 were shown to cooperate for effective ligand binding (Cohen et al. 1995), several type I interferons have different affinities for either of the receptor subunits (Jaks et al. 2007). In this regards, it was shown that IFN $\beta$ can bind to IFNAR1 in an IFNAR2-independent manner (de Weerd et al. 2013). Therefore, it was investigated whether IFNAR2 participates in the IFNAR1mediated regulation of microglial chemokine production. Because no IFNAR2 knock-out mice were available, an antibody was used to functionally block IFNAR2 in wt cells. Similar to the IFNAR1 blockade, application of an $\alpha$-IFNAR2 antibody increased the CXCL1 and decreased the CCL2 production by microglia. With this approach, we could demonstrate that IFNAR2 is also involved in regulating the TLR4- and TLR2-induced chemokine release.

So finally, we wanted to investigate whether both receptor subunits cooperate for the regulation of microglial chemokine production. In order to create an IFNAR1/IFNAR2-deficient system, ifnar $1^{-1}$ microglia were treated with the IFNAR2-blocking antibody. The CXCL1 and CCL2 release of these double-deficient cells was compared to IFNAR1-deficient, IFNAR2-blocked and wt microglia. In the absence of IFNAR1 and IFNAR2 simultaneously, effects on TLR4- and TLR2-triggered CXCL1 levels were even stronger than in individual deficiency. The already increased production in ifnar $1 \%$ compared to wt cells could be further enhanced by IFNAR2-blockade. In contrast to that, no additional effect of IFNAR2-blockade on CCL2 release by ifnar $1^{\%}$ microglia could be observed. While CCL2 levels were lower in ifnar $1^{\%}$ compared to wt cells, this could be not further decreased with the $\alpha$-IFNAR2 antibody. Possibly, the CCL2 production by ifnar ${ }^{\%}$ microglia was already as low as achievable. Nevertheless, the results concerning CXCL1 demonstrated a cooperative regulation by IFNAR1 and IFNAR2.

All in all, we could show that IFNAR with its both subunits IFNAR1 and IFNAR2 is critical for the regulation of chemokine production. This is the case for microglia, but also other tissue-resident macrophages. In particular, CXCL1 and CCL2 release in response to TLR stimulation were controlled by this receptor. Thus, the canonical type I interferon signaling pathway could be essential for this regulatory mechanism.

\subsection{IFNAR1 regulates the infiltration of neutrophils in a model of gram-negative, but not gram-positive meningitis}

Meningitis is an acute inflammation of the meninges that can be life-threatening, especially in neonates. In 2013, 16 million people worldwide suffered from meningitis (Global Burden of Disease Study 2013 Collaborators 2015) of which 303,000 died (GBD 2013 Mortality and Causes of Death Collaborators 
2015). The disease is typically caused by infectious agents, including several bacteria. In neonates, the most common meningeal pathogens are group B streptococci and E. coli K1. Infants and small children are mostly affected by S. pneumoniae, Neisseria meningitidis and Haemophilus influenzae type $\mathrm{B}$, while in children older than 5 years and adults bacterial meningitis is almost exclusively caused by S. pneumoniae and Neisseria meningitidis (Sáez-Llorens and McCracken 2003).

The meninges are in direct contact with the brain and therefore, bacterial constituents can reach the brain tissue leading to a large-scale inflammation. Such inflammatory reactions involve microglia as the tissue-resident immune cells, which can sense the presence of bacterial components by recognition of PAMPs, for example through TLRs (Barichello et al. 2016). Activation of these receptors triggers the production of several cytokines and chemokines with subsequent recruitment of peripheral immune cells into the CNS (Gerber and Nau 2010). As a first line of defense, monocytes and neutrophils are rapidly recruited to the site of inflammation. Thereby, monocytes follow chemotactic gradients of CCL2 and CCL7 (Tsou et al. 2007), while neutrophils migrate along CXCL1 and CXCL2 gradients (Wengner et al. 2008).

As a model of gram-negative meningitis, infection was induced by intracerebral injection of E. coli K1. Similarly, gram-positive meningitis was evoked by S. pneumoniae D39 injection. While E. coli contain LPS within their cell wall, which is sensed by TLR4 (Hoshino et al. 1999), peptidoglycan and lipoteichoic acid as cell wall components of S. pneumoniae are recognized by TLR2 (Yoshimura et al. 1999; Mogensen et al. 2006). Because we could show that following stimulation of TLR4 or TLR2, microglial production of the neutrophil chemoattractant CXCL1 and the monocyte chemoattractant CCL2 in vitro is regulated by IFNAR, we hypothesized that this receptor also controls the in vivo recruitment of these cells accordingly. Therefore, we compared wt and ifnar $1^{-/}$mice concerning their infiltration of monocytes and neutrophils into the brain after intracerebral injection of E. coli or S. pneumoniae. Because both infections have a different latency period until the onset of disease symptoms, E. coli-injected mice were analyzed $24 \mathrm{~h}$ and S. pneumoniae-injected animals $36 \mathrm{~h}$ after the infection.

In E. coli-infected wt and ifnar ${ }^{-/}$mice, substantial amounts of inflammatory monocytes and neutrophils infiltrated the brain after $24 \mathrm{~h}$. While no difference between both genotypes regarding monocyte numbers was observed, significantly more neutrophils were detected in the brains of ifnar $1^{1 /}$ mice. Similar results were obtained in response to sterile axotomy, where IFNAR1 deficiency increased the infiltration of leukocytes into the brain (Khorooshi and Owens 2010) and in a model of septic peritonitis, in which peritoneal neutrophil recruitment and activation was augmented in ifnar $1 \%$ mice (Weighardt et al. 2006). In our E. coli meningitis model, the elevated neutrophil numbers mirror our in vitro data that revealed a massive overproduction of the neutrophil chemoattractant CXCL1 by TLR4-stimulated microglia in the absence of IFNAR1. In contrast to that, the slightly decreased CCL2 production by ifnar $1^{-/}$microglia was not reflected in reduced monocyte numbers. This could be attributed to the fact that monocytes do not only follow CCL2, but also CCL7 gradients (Tsou et al. 2007).

Importantly, this difference in the recruitment of neutrophils could not be caused by an exacerbated disease progression due to impaired microglial phagocytosis in ifnar $1^{\text {- }}$ mice. Microglia are capable of phagocytosing different nonencapsulated and encapsulated bacteria, including E. coli (Kaur et al. 2004; Ribes et al. 2009) and S. pneumoniae (Ribes et al. 2010). This ability is a powerful mechanism to fight against brain infections, such as meningitis (Sierra et al. 2013). If microglia are not able to properly phagocytose the invading E. coli, this could lead to multiplication of the bacteria and thus, to a spread of the infection (Ribes et al. 2009). Accordingly, this would result in an increased recruitment of peripheral immune cells in order to cope with the infection. However, we demonstrated that ifnar $1^{-/}$microglia have the same capacity as wt cells to phagocytose pathogens, represented by E. coli and endogenous 
material, represented by myelin. This is in line with previous reports showing that the phagocytosis of bacteria by macrophages is not altered by IFNAR deficiency (Kelly-Scumpia et al. 2010; Rathinam et al. 2012). Thus, it appears to be really the IFNAR-dependent regulation of TLR4-induced CXCL1 that makes the difference in neutrophil recruitment during E. coli meningitis.

As the production of CXCL1 in response to TLR2 stimulation was also increased by IFNAR1 deficiency, we hypothesized that the recruitment of neutrophils during S. pneumoniae meningitis would be enhanced similar to the E. coli model. However, already the amount of recruited monocytes and neutrophils was markedly lower in S. pneumoniae- than E. coli-infected mice. Even though more cells compared to untreated animals or PBS controls were observed, these numbers did not reach statistical significance. One reason for that is the high variation between individual animals. While E. coli-infected mice showed a relatively homogeneous disease severity as determined by bacteremia, S. pneumoniae infection was much more variable with a markedly higher mortality rate (data not shown). Within the first $36 \mathrm{~h}$, this mortality rate did not differ between wt and ifnar ${ }^{\%}$ mice (data not shown), indicating no difference in disease susceptibility in our short-term model. Considering long-term survival studies, results concerning the influence of IFNAR1 on resistance against $S$. pneumoniae are, however, controversial (Mancuso et al. 2007; Mitchell et al. 2012). Apart from the high variation of the disease outcome, another reason for the low amount of infiltrating immune cells might lie in the time point. In this regard, $36 \mathrm{~h}$ could prove to be suboptimal. As shown by Janova et al. (2016), monocyte and neutrophil numbers in the brain peak $24 \mathrm{~h}$ after intracerebral $E$. coli injection and subsequently decrease again. Additionally, the leukocyte density in the CSF of $S$. pneumoniae-infected mice is also highest $24 \mathrm{~h}$ after the injection and already lower at the $36 \mathrm{~h}$ time point, even though disease symptoms are worst after $36 \mathrm{~h}$ (Gerber et al. 2001). However, it was necessary to wait $36 \mathrm{~h}$ instead of $24 \mathrm{~h}$ for the animals to show disease symptoms, because the $S$. pneumoniae infection has a higher latency. Nevertheless, also the overall lower amounts of infiltrating cells allowed a comparison of wt and ifnar $1 \%$ mice. This revealed no difference in monocyte and neutrophil numbers between both genotypes. Hence, the overproduction of CXCL1 by TLR2-stimulated ifnar $1 \%$ microglia did not correlate with the neutrophil recruitment in the S. pneumoniae meningitis model. A possible explanation for this could be that $S$. pneumoniae do not only activate TLR2, but also TLR4 and TLR9 (Mogensen et al. 2006; Lee et al. 2007). Furthermore, NOD2 contributes to sensing of $S$. pneumoniae by intracellular recognition (Opitz et al. 2004). Thus, the recruitment of neutrophils could be organized more complex than simply depending on TLR2-induced chemokine production. In this context, also the release of CXCL2 as neutrophil chemoattractant could play a crucial role. IFNAR1-deficient microglia that were stimulated with Pam3CSK4 or MALP-2 for activating the TLR2 complex did not produce more CXCL2 than wt cells (data not shown). This indicates that CXCL2 in contrast to CXCL1 release is not controlled by IFNAR1. Therefore, neutrophils could also follow an IFNAR1-independent gradient of CXCL2 during S. pneumoniae meningitis.

In summary, we identified IFNAR1 as important regulator of gram-negative CNS infection. In a model of $E$. coli meningitis, controlled infiltration of neutrophils was dependent on the presence of IFNAR1. In contrast to that, gram-positive S. pneumoniae meningitis seems to be organized independent of type I interferon signaling.

\subsection{The infiltration of neutrophils into focal NMO-like lesions is independent of IFNAR1}

NMO is a chronic inflammatory demyelinating disease of the CNS. In contrast to bacterial infections, such as meningitis, the inflammation is in this case not caused by invading pathogens, but linked to 
autoantibodies directed against AQP4 (Lennon et al. 2005). These serum autoantibodies have been found in about $60-80 \%$ of the NMO patients (Jarius et al. 2011). Because in the CNS AQP4 is mainly expressed on astrocytes (Nagelhus et al. 2004), these cells are the primary target of the autoantibodies. Thus, NMO lesions are characterized by extensive loss of astrocytes (Roemer et al. 2007; Misu et al. 2007) with subsequent reduction of oligodendrocyte numbers and demyelination (Lucchinetti et al. 2002; Wrzos et al. 2014).

In our model, focal NMO-like lesions were induced by stereotactic injection of a human recombinant anti-AQP4 antibody (NMO-Ab) together with human complement into the cortex of mice. In accordance with other studies in mice (Saadoun et al. 2010) and rats (Wrzos et al. 2014), the injection resulted in astrocyte depletion and lesion formation. This process was accompanied by infiltration of immune cells. As described by Lucchinetti et al. (2002), inflammatory infiltrates within human NMO lesions consist of monocytes, macrophages, lymphocytes and polymorphonuclear cells, such as neutrophils, basophils and eosinophils. Similar observations were made in animal models of focal NMO (Asavapanumas et al. 2014; Wrzos et al. 2014). According to that, we were able to detect substantial amounts of neutrophils within the lesions $24 \mathrm{~h}$ after the injection.

Because we could show that the infiltration of neutrophils in a model of gram-negative meningitis is controlled by IFNAR1, we hypothesized that a similar regulation could also apply to other inflammatory conditions. In this regard, NMO is a very interesting model as it is no bacterial infection, but an autoimmune disorder. For dissecting the role of IFNAR1 in this context, we compared the infiltration of neutrophils into focal NMO-like lesions of wt and ifnar $1 \%$ mice by using two different approaches. On the one hand, neutrophils were counted in CAE-stained sections and on the other hand, they were detected in whole brain single-cell suspensions by flow cytometry. Even though neutrophil numbers were in general rather low compared to the meningitis model, more infiltrating cells were observed in NMO-Ab-injected animals than in PBS-injected controls. However, there was no difference between wt and ifnar ${ }^{\%}$ mice. Analysis of inflammatory monocytes and patrolling monocytes revealed also similar numbers in both genotypes. Thus, immune cell infiltration into focal NMO-like lesions was not altered by IFNAR 1 deficiency in our model. At first glance, this seems to be in contrast to a study by Khorooshi et al. (2013), which showed a dependence of NMO-like pathology on type I interferon responses. They observed a reduced NMO histopathology in IFNAR1-deficient mice that also manifested in reduced numbers of infiltrating granulocytes 4 days after lesion induction. However, the different timing is most probably the point that makes the difference. At 2 days after lesion induction, no significant changes in granulocyte numbers of ifnar $1 \%$ compared to wt mice were observed in this study. Considering that we already analyzed neutrophil numbers after $24 \mathrm{~h}$, this early infiltration proves to be independent of IFNAR1.

An important mechanism to guide immune cells into damaged tissue is the establishment of chemotactic gradients (Hesselgesser and Horuk 1999). The migration of neutrophils is thereby mainly determined by CXCL1 and CXCL2 (Wengner et al. 2008). These chemokines are rapidly upregulated under several neuropathologic conditions (Omari et al. 2006; Semple et al. 2010; Johnson et al. 2011), including NMO (Herges et al. 2012). According to that, we observed elevated intracerebral CXCL1 levels in mice with focal NMO-like lesions. A comparison of wt and ifnar $1^{\%}$ animals $24 \mathrm{~h}$ after lesion induction revealed a similarly increased production, indicating no influence of type I interferon signaling on CXCL1 levels in the lesions. This correlated with the fact that early neutrophil infiltration was independent of IFNAR1. However, it contrasts our findings concerning CXCL1 production in response to TLR4 and TLR2, where we demonstrated an IFNAR-dependent control. Accordingly, involvement of type I interferon signaling 
in the regulation of CXCL1 levels might be highly dependent on the pathway that triggers the chemokine production.

Apart from CXCL1 and CXCL2, the anaphylatoxin C5a acts as potent neutrophil chemoattractant (Ward and Newman 1969; Fernandez et al. 1978). C5a is a protein fragment that is released upon activation of the complement cascade (Nesargikar et al. 2012). During NMO, the complement cascade is initiated by binding of the autoantibodies to AQP4 on astrocytes leading to complement-mediated astrocyte damage, followed by granulocyte infiltration and oligodendrocyte death (Papadopoulos and Verkman 2012). It was shown in human NMO lesions that the complement cascade is extensively activated (Lucchinetti et al. 2002) and thus, the presence of C5a could lead to the recruitment of neutrophils. This is supported by the fact that C5a levels were found to be significantly elevated in the CSF of NMO patients (Kuroda et al. 2013). Hence, attraction of neutrophils to NMO lesions might depend on activation of the complement cascade rather than CXCL1 production.

Overall, our results indicate mechanistic differences in immune cell attraction during different diseases of the CNS. While IFNAR1 was demonstrated to be an important regulator of neutrophil recruitment in a model of gram-negative meningitis, it had no influence on this process in a model of focal NMO. In this regard, especially the involvement of TLRs and subsequent CXCL1 production seems to be the key issue concerning the influence of IFNAR1.

\subsection{Janus kinases control chemokine production in response to TLR4, but not TLR2}

Janus kinases are a family of intracellular, non-receptor tyrosine kinases. This family comprises four members, namely Jak1, Jak2, Jak3 and Tyk2. They are involved in signaling cascades of receptors that use the so-called Jak-STAT pathway for their signal transduction (Ghoreschi et al. 2009). One of these receptors is IFNAR and accordingly, Jak1 and Tyk2 are the central kinases within the canonical type I interferon signaling pathway. Upon binding of type I interferons to IFNAR, they initiate a tyrosine phosphorylation cascade that ultimately leads to the activation of STAT transcription factors and expression of ISGs (Platanias 2005).

We demonstrated that IFNAR is an important regulator of TLR-induced chemokine production. In particular, CXCL1 and CCL2 levels were shown to be opposingly affected by IFNAR deficiency. Considering that IFNAR mainly exerts its effects via the canonical type I interferon signaling, Jak1 and Tyk2 could be important mediators of the IFNAR-dependent control of these chemokines.

In order to get an overview about a possible influence of janus kinases in general, the kinase activity of all family members was blocked. Therefore, microglia were stimulated with agonists for TLR4 and TLR2 to induce CXCL1 and CCL2 production in the presence of Jak inhibitor I. This compound is a reversible, ATP-competitive janus kinase inhibitor, which potently blocks kinase activity with an $\mathrm{IC}_{50}$ value of $15 \mathrm{nM}$ for Jak1 and $1 \mathrm{nM}$ for Tyk2 (Thompson et al. 2002). In response to Re-LPS as TLR4 agonist, Jak inhibitor I dose-dependently decreased CCL2 and increased CXCL1 production. Interestingly, so far mostly negative regulatory effects of janus kinase inhibitors on different cytokines and chemokines were observed. Similar to our results regarding Re-LPS-induced CCL2, Jak inhibitor I was shown to decrease dsRNA-induced production of CXCL10 and CCL5 in microglia (Nakamichi et al. 2005). Furthermore, the Jak1 / Jak2 inhibitor Ruxolitinib reduced the production of IL-12 by dendritic cells (Heine et al. 2013) and the Jak1 / Jak3 inhibitor Tofacitinib suppressed synovial expression of CCL2, CXCL10 and CXCL13 in patients with rheumatoid arthritis (Boyle et al. 2015). Consequently, the marked increase that we 
observed in CXCL1 production upon treatment with Jak inhibitor I seems to be rather unique. Nevertheless, this reflects our observations in an IFNAR-deficient system, indicating that indeed janus kinases mediate the regulatory effect of IFNAR. However, even though Jak inhibitor I was claimed to be highly potent (Williams et al. 2009), a concentration of $100 \mathrm{nM}$, which by far exceeds the above mentioned $I_{50}$ values, was necessary to obtain significant differences in CXCL1 and CCL2 levels. Nevertheless, a high maximal effect on both chemokines could be achieved. In response to MALP-2 as TLR2 agonist, a dose-dependent decrease of CXCL1 production with a similar potency, but much lower maximal effect than in the TLR4 system was observed. This trend is in contrast to our results regarding IFNAR, whose deficiency consistently increased TLR2-triggered CXCL1 levels. Interestingly, Jak inhibitor I had no dose-dependent effect on Pam3CSK4-induced CXCL1, but increased its production at seemingly random concentrations. Thus, the regulation of TLR2-induced chemokine production is most probably independent of janus kinases and the observed findings are attributed to off-target effects.

All janus kinases share the same structure. They consist of seven Jak homology $(\mathrm{JH})$ regions that contain a kinase domain (JH1), a pseudokinase domain (JH2), a SH2 domain (JH3, JH4), and a fourpoint-one, ezrin, radixin, moesin (FERM) domain (JH6, JH7) (Ghoreschi et al. 2009). The enzymatic activity resides within the kinase domain, while the catalytically inactive pseudokinase domain has only regulatory functions (Chen et al. 2000; Saharinen and Silvennoinen 2002). The janus kinase SH2 domain is structurally important, but does not have its classical phospho-tyrosine-binding capability (Radtke et al. 2005) and the FERM domain mediates protein-protein interactions, linking janus kinases with their cognate transmembrane cytokine receptor proteins (Tanner et al. 1995; Girault et al. 1998). This overall high structural similarity is also reflected in the kinase domain that shares about $50 \%$ sequence identity (Williams et al. 2009). According to that, it is very difficult to design kinase inhibitors that specifically target only one member of the janus kinase family. In order to dissect the individual role of Jak1 and Tyk2 in the regulation of TLR-induced chemokine production, we used GLPG0634. This inhibitor is more selective for Jak1 than Tyk2 as indicated by the $\mathrm{IC}_{50}$ values of $10 \mathrm{nM}$ for Jak1 and $116 \mathrm{nM}$ for Tyk2 (Van Rompaey et al. 2013). In comparison to Jak inhibitor I, GLPG0634 had a reduced potency and lower maximal effect on the production of CXCL1 and CCL2 in response to Re-LPS. Only the highest concentration significantly increased CXCL1, so that no dose-dependency could be shown. Concerning the reduction of CCL2 levels, which was already described previously (Van Rompaey et al. 2013), the potency was higher, but overall, the dose-response curves did not show a continuous curve progression. On the one hand, this markedly lower effect of GLPG0634 could be attributed to the fact that it more selectively targets Jak1, while Jak inhibitor I blocks Jak1 and Tyk2 relatively equal. This would point towards a cooperative effect of both kinases in the regulation of CXCL1 and CCL2 production. On the other hand, biochemical properties of the compound and correspondingly lower biological activity could be an explanation. Thus, we could not conclude on a selective impact of Jak1 on TLR4-induced chemokine production. Regarding TLR2-triggered CXCL1 levels, GLPG0634 had no systematic effect. While it increased CXCL1 production in response to Pam3CSK4 at some random concentrations, it had no effect on MALP-2 -induced release. So similar to Jak inhibitor I, we could not show an involvement of janus kinases in IFNAR-dependent control of CXCL1 levels in response to TLR2.

For finally investigating the role of Jak1 in regulating TLR4- versus TLR2-induced chemokines, we took advantage of a new compound that is a Jak1-specific inhibitor, namely Solcitinib (Kahl et al. 2016). Because of its novelty, so far no $\mathrm{IC}_{50}$ value was published. When applying this inhibitor to Re-LPSstimulated microglia, it proved to be highly potent and effective in increasing CXCL1 and decreasing CCL2 levels in a dose-dependent manner. In contrast to this, no effect on TLR2-induced CXCL1 production was observed. So by using this compound, we could demonstrate that Jak 1 specifically participates in the control of TLR4-, but not TLR2-induced chemokine production. Unfortunately, these results 
could not be verified in a Jak1 knock-out system, because jak $1^{-/}$mice fail to nurse and die perinatally (Rodig et al. 1998).

By using different inhibitors, we could show that janus kinases and in particular Jak1 are important regulators of CXCL1 and CCL2 production in response to TLR4 stimulation. This links the IFNAR-dependent control of these chemokines with the key kinases of the canonical type I interferon signaling pathway. Nevertheless, a direct proof for an involvement of Tyk2 in this scenario was missing. Because no Tyk2specific inhibitor was available, a Tyk2-deficient mouse strain should be used in order to investigate the individual role of this kinase. As we did not have access to tyk2\% mice (Shimoda et al. 2000), we used a mouse strain with a naturally occurring missense mutation in the tyk2 gene (tyk2 $\left.{ }^{E 775 K}\right)$. This mutation is located in an invariant motif of the pseudokinase domain within the JH2 region (Shaw et al. 2003), which is required for Tyk2 activation by phosphorylation and also for its kinase activity (Velazquez et al. 1995). Accordingly, no active Tyk2 can be detected in tyk2 ${ }^{E 775 K}$ mice (Shaw et al. 2003). Therefore, tyk $2^{E 775 K}$ mice served as a model of Tyk2 deficiency.

Interestingly, tyk2 ${ }^{E 775 K}$ microglia showed totally unexpected properties. Because overall blockade of janus kinases by Jak inhibitor I resulted in increased CXCL1 and decreased CCL2 levels in TLR4stimulated cells, we hypothesized a similar pattern in tyk2 ${ }^{E 775 K}$ microglia. However, we observed completely opposite results as the Tyk2 missense mutation resulted in increased CCL2 and decreased CXCL1 production. This was not only true for TLR4-, but also for TLR2-induced responses. Considering that none of the tested janus kinase inhbitors dose-dependently altered TLR2-triggered CXCL1 release, this was even more surprising. A possible explanation for these unexpected findings could lie in altered IFN $\beta$ production. The presence of high IFN $\beta$ levels decreases Re-LPS- or FN-induced CXCL1 release dramatically (Janova et al. 2016). Furthermore, IFN $\beta$ triggers CCL2 production even in the absence of any TLR agonist (McManus et al. 2000; Fantuzzi et al. 2001), which we also observed in $t y k 2^{E 775 K}$ microglia (data not shown). So overall, higher IFN $\beta$ levels in tyk2 ${ }^{E 775 K}$ microglia could cause the observed decrease in CXCL1 and increase in CCL2. However, the IFN $\beta$ production was even slightly reduced in $t y k 2^{E 775 K}$ microglia, indicating that another mechanism accounts for this phenomenon. Surprisingly, we could even show that $t y k 2{ }^{E 775 K}$ microglia react to high amounts of exogenous IFN $\beta$ by decreasing CXCL1 levels similar to wt cells, which argues for a functional IFN $\beta$-mediated regulatory feedback. Thus, type I interferon signaling is not completely disrupted by the Tyk2 missense mutation. In line with that, it was shown that type I interferon responses are only partially impaired in Tyk2-deficient mice (Karaghiosoff et al. 2000; Shimoda et al. 2000). A possible explanation could lie in still functional Jak1. It was shown that tyk2 ${ }^{E 775 K}$ cells express normal levels of Jak2 protein and exhibit a Jak2 phosphorylation response (Shaw et al. 2003), so most probably the same also applies to Jak1. Accordingly, Jak1 could mediate an IFN $\beta$-induced regulation of CXCL1 production independent of Tyk2. This would indicate that either both kinases do not need to cooperate for signal transduction or that Jak1 functionally dominates over Tyk2. In order to prove such a possibility, janus kinases were completely blocked by using Jak inhibitor I. With this inhibitor, Jak1 as well as possibly remaining Tyk2 kinase activity should be disrupted. However, even the highest concentration did not affect the really low CXCL1 production in both, TLR4- and TLR2-stimulated tyk2 ${ }^{E 775 K}$ microglia. Similar results were obtained by using Solcitinib as selective Jak1 inhibitor. Taken together, this data argues for a general impairment of CXCL1 production in tyk2 ${ }^{E 775 K}$ microglia. In line with that, CXCL1 expression in an anti-type II collagen antibody-induced arthritis model (Ishizaki et al. 2011) as well as an IL-23-induced psoriasis-like skin inflammation model (Ishizaki et al. 2014) was significantly lower in $t y k 2^{-/}$than wt mice. Furthermore, selectivity of the CXCL1 impairment is supported by the fact that CCL2 production by tyk2 ${ }^{E 775 K}$ microglia could be dose-dependently decreased by Jak inhibitor I and Solcitinib similar to wt cells. However, inconsistent with the previously described results, wt and tyk2 ${ }^{E 775 K}$ cells showed a 
comparable CCL2 production in these experiments. When comparing this to the literature, Tyk2 knockout, in contrast to the Tyk2 missense mutation, even results in decreased expression of CCL2 under inflammatory conditions (Ishizaki et al. 2011; Ishizaki et al. 2014). Nevertheless, this demonstrates that tyk $2^{E 775 K}$ microglia are in principle sensitive to janus kinase inhibition and wt levels of CCL2 can be restored.

Overall, it could not be clarified whether Tyk2 participates in mediating the IFNAR-dependent control of TLR-induced chemokine production by using $t y k 2^{E 775 K}$ microglia. On the one hand, the obtained results would indicate that Tyk2 is not crucial, because the Tyk2 impairment was not sufficient to decrease CCL2 levels, but rather complete janus kinase blockade or Jak1 inhibition were required. However, no conclusions on CXCL1 production could be drawn. On the other hand, the necessity of janus kinase blockade for obtaining a regulatory effect could also indicate that Tyk2 is still functional in tyk2 ${ }^{E 775 K}$ microglia. Even though it was reported that no active Tyk2 can be detected in these cells, the mutation only affects the pseudokinase domain (Shaw et al. 2003). This could result in residual kinase activity that interferes with our results. Accordingly, blockade of Tyk2 kinase activity would cause the decreased CCL2 production and consequently, Tyk2 could be important for mediating effects of IFNAR on chemokine production. Furthermore, it has to be considered that probably also protein-protein interactions of Tyk2 are still present in tyk2 ${ }^{E 775 K}$ cell, which have a completely functional FERM domain. This is underlined by the fact that only the FERM domain, stabilized by JH3-5, is required for Tyk2 binding to IFNAR1 (Richter et al. 1998). Hence, Tyk2 could exert regulatory effects, apart from acting as kinase, by interacting with other proteins. Thus, microglia carrying a missense mutation in the tyk 2 gene did not serve as a good tool for investigating the role of Tyk2 in an IFN $\beta$-mediated IFNAR-dependent regulatory feedback.

In summary, we demonstrated by using different kinase inhibitors that CXCL1 and CCL2 levels in response to TLR4 stimulation are regulated by janus kinases and in particular Jak1. This is in line with our observations regarding IFNAR as regulator of these chemokines. In contrast to that, the TLR2 system was shown to be mainly independent of these kinases, even though CXCL1 levels were controlled by IFNAR. Thus, janus kinases selectively mediate IFNAR-dependent effects on different TLR systems.

\subsection{STAT1 and STAT2 control chemokine production in response to TLR4 and TLR3, but not TLR2}

STAT proteins are a group of transcription factors that transmit extracellular signals into the nucleus. They are mainly activated by membrane receptor-associated janus kinases and thus, serve as essential mediators within Jak-STAT signaling (Darnell et al. 1994). So far, seven different mammalian STAT proteins have been identified: STAT1, STAT2, STAT3, STAT4, STAT5A, STAT5B and STAT6 (Shuai and Liu 2003). They were first described for their involvement in the interferon system (Fu et al. 1992; Shuai et al. 1993), but also participate in signal transduction of other cytokine receptors and growth factors (Sadowski et al. 1993).

Downstream of IFNAR, several STATs become activated. STAT1 and STAT2 are the essential mediators of the canonical type I interferon signaling, but STAT3 and STAT5 can be also involved in type I interferon responses (Yang et al. 1996; Meinke et al. 1996). Upon activation, STAT1 and STAT2 heterodimerize and recruit IRF9 in order to form the ISGF3 complex (Martinez-Moczygemba et al. 1997). This complex binds to ISREs in the promoter of ISGs and thereby regulates gene transcription (Schindler et al. 1992). 
Following activation of different TLRs, several interferons are released leading to activation of type I interferon signaling. The striking cross-talk of both pathways is illustrated by the fact that several TLRmediated functions are not generated in the absence of IFNAR (Hwang et al. 1995; Hamilton et al. 1996). Apart from that, involvement of type I interferon signaling in TLR-induced responses can be demonstrated by activation of STAT proteins as crucial transcription factors within this pathway (Ohmori and Hamilton 2001). Upon stimulation of TLR4 by LPS, STAT1 becomes activated as shown by tyrosine phosphorylation (Toshchakov et al. 2002). This holds also true for TLR3 activation by poly I:C, but not for the TLR2 agonist Pam3CSK4 (Rhee et al. 2003; Luu et al. 2014). In line with these findings, we observed up-regulation of STAT1 gene expression in microglia stimulated with Re-LPS or FN, but not Pam3CSK4 or MALP-2. However, since STAT1 also participates in responses to IFN $\gamma$, the activation of this protein is not necessarily specific to type I interferon signaling (Platanias 2005). Therefore, we also measured the expression of STAT2, which rather specifically regulates transcription in response to type I, but not type II interferons (Shuai et al. 1992). This analysis revealed a similar up-regulation of STAT1 and STAT2 expression in TLR4-, but not TLR2-stimulated microglia. Accordingly, STAT1/2 heterodimers as essential transcriptional regulators of type I interferon signaling could participate in TLR4-mediated responses.

For this reason, STAT1 and STAT2 would be promising candidates for meditating the IFNAR-dependent and janus kinase-mediated regulation of microglial CXCL1 and CCL2 production in response to TLR4, but not TLR2. In order to test this hypothesis, stat $1^{-/}$and stat $^{-/}$microglia were analyzed concerning their chemokine release triggered by TLR4, TLR2 and additionally also TLR3 stimulation.

Within the TLR2 system, deficiency in STAT1 or STAT2 did not affect microglial CXCL1 production, which is in line with the previously shown absence of STAT1 involvement in TLR2-mediated responses (Toshchakov et al. 2002; Rhee et al. 2003) and our observations concerning gene expression. Furthermore, this reflects our results regarding janus kinases. Since they most probably do not participate in responses to TLR2 as indicated by chemokine production independent of these kinases, they could also not activate the downstream transcription factors STAT1 and STAT2.

In contrast to that, TLR3-triggered responses were altered by STAT1 and STAT2 deficiency. CCL2 production was decreased in stat $1^{\%}$ and stat $2^{-/}$compared to wt microglia. However, also IFN $\beta$ levels in response to poly I:C were significantly lower in the absence of STAT1 or STAT2. As already described in chapter 5.2, this could be causative for alterations in CCL2 release. IFN $\beta$ is a potent inducer of this chemokine (McManus et al. 2000; Fantuzzi et al. 2001), which we also observed in stat $1^{-/}$and stat $^{-/}$ microglia (data not shown). Furthermore, impaired IFN $\beta$ production correlates with dysregulation of CCL2 (Janova et al. 2016). So similar to what was described for IFNAR1 deficiency, it could not be concluded whether altered CCL2 release by poly I:C-stimulated stat $1^{-/}$and $s t a t 2^{-/}$microglia is due to deficiency in the respective transcription factor or caused by the low IFN $\beta$ levels.

Concerning responses to TLR4, it was already indicated that STAT1 and STAT2 play an important role as stated above. Indeed, this could be proven in both knock-out systems. In line with our observations regarding IFNAR and janus kinases, STAT1 and STAT2 deficiency resulted in increased CXCL1 and decreased CCL2 production. However, a partially impaired IFN $\beta$ release was present, but only in stat $1^{-1}$ microglia. Thus, we had to rule out that alterations in IFN $\beta$ levels affect the chemokine release by these cells. Therefore, we evened out these differences by addition of high amounts of exogenous IFN $\beta$, which by far exceeded the intrinsic microglial production. This demonstrated that only wt, but neither stat $1 \%$ nor stat $\%$ microglia were highly sensitive to this treatment. While addition of IFN $\beta$ dramatically decreased CXCL1 levels in wt cells, the production by stat $1 \%$ and stat ${ }^{\%}$ stayed markedly higher than normal wt levels even in the presence of IFN $\beta$. Since the effect of STAT deficiency on CXCL1 was really stable, this proves that the minor impairment of IFN $\beta$ in stat $1 \%$ cells cannot account for the observed 
changes in CXCL1 and CCL2 production. This argues for a STAT1- and STAT2-dependent control of these chemokines.

Nevertheless, we had to consider that no STAT1 / STAT2 double knock-out was available and analyzing deficiency in only one of the transcription factors bears the risk of compensation effects. Such a phenomenon could lead to still, at least partially, functional transcriptional regulation and would thus interfere with our results obtained in stat $1^{-}$and stat $2^{\%}$ microglia. Besides its ability to form heterodimers with STAT1, STAT2 is also able to heterodimerize with STAT3 (Hervas-Stubbs et al. 2011). Because it was shown that STAT3 can be also involved in type I interferon signaling (Yang et al. 1996), we had to rule out involvement of this transcription factor in regulation of TLR-induced microglial responses. Thus, STAT3 expression upon either TLR4 or TLR2 stimulation was measured. In contrast to STAT1 and STAT2, its expression was not up-regulated in response to TLR stimulation, indicating that STAT3 is not activated in this scenario. Apart from that, we had to take into account that STAT1 can also form homodimers (Hervas-Stubbs et al. 2011). This provides the potential for autonomous actions of STAT1 in the absence of STAT2. Such a compensation would be accompanied by up-regulation of the stat 1 gene in STAT2-deficient cells. However, the expression STAT1 could not be up-regulated in stat2\% microglia by either TLR4 or TLR2 stimulation. The same holds true for STAT2, whose expression remained also unchanged in stat ${ }^{-}$cells. This strongly suggests that STAT1 and STAT2 need to cooperate in order to exert effects in TLR-stimulated microglia.

Finally, these results clearly show an involvement of STAT1 and STAT2 in responses to TLR4 and accordingly, both transcription factors are important mediators of the IFNAR-dependent and janus kinasemediated regulation of TLR4-triggered chemokine production. In contrast to that, they do not participate in responses to TLR2 and their contribution to TLR3-induced CCL2 release remains still questionable.

\subsection{Type I interferon signaling controls TLR4-induced chemokine production, while responses to TLR2 are regulated by IFNAR}

Altogether, we showed that microglial responses to gram-positive and gram-negative bacteria are individually organized. The TLR2 and TLR4 system as exemplary examples for cell surface receptors that sense bacterial ligands differently utilize parts of the canonical type I interferon signaling pathway for regulating the production of chemokines. Thus, we want to propose two different mechanisms how type I interferon signaling controls TLR-induced responses.

TLR2 can be activated by bacterial ligands, such as Pam3CSK4 or MALP-2 (Jin et al. 2007; Takeuchi et al. 2001). Subsequently, the MyD88-dependent signaling pathway is initiated, which leads to the production of several cytokines and chemokines (Kawai and Akira 2010). 18 h after stimulation of the receptor, this includes CXCL1, but not CCL2. In order to control the amount of released CXCL1, another signaling pathway gets involved. We demonstrated that both subunits of the type I interferon receptor cooperatively regulate TLR2-induced CXCL1 production. However, we could not identify the respective ligand, since neither IFN $\alpha$ nor IFN $\beta$ was produced upon TLR2 stimulation. One possible explanation could be low sensitivity of our methods. More likely, the release of another type I interferon apart from IFN $\alpha$ or IFN $\beta$ is triggered by TLR2. Even though we could not identify the respective ligand, IFNAR becomes activated in this scenario. Downstream of IFNAR, several signaling pathways could be triggered. The most prominent is the canonical type I interferon signaling pathway, which involves the kinases Jak1 and Tyk2 as well as the transcription factors STAT1 and STAT2 (Platanias 2005). However, we did not 
find any of these mediators to control TLR2-induced chemokine production. Thus, the canonical type I interferon signaling pathway seems not to play a role in responses to TLR2. Alternatively, the CRKL, MAPK or PI3K pathway could be activated by IFNAR (Hervas-Stubbs et al. 2011). However, since they all require Jak1 and/or Tyk2 for signal transduction (Platanias 2005) and we could not observe involvement of janus kinases in general, it is unlikely that one of these pathways is triggered. However, no janus kinase-independent pathway downstream of IFNAR could be identified. Thus, it remains enigmatic how IFNAR regulates responses to TLR2.

In contrast to that, the mechanism for controlling TLR4-induced chemokine levels can be explained considerably better. TLR4 gets activated by the bacterial ligand LPS and also by the DAMP FN (Poltorak et al. 1998; Hoshino et al. 1999; Okamura et al. 2001). Upon activation, TLR4 triggers the MyD88- and the TRIF-dependent signaling pathway (Kawai and Akira 2010). In order to initiate the TRIF-dependent signaling, the TLR4 complex is internalized by CD14-induced endocytosis (Kagan et al. 2008; Zanoni et al. 2011). The activation of both signaling pathways results in the production of CXCL1 and CCL2 as well as IFN $\beta$. Thereby, as shown by us and also others, the production of IFN $\beta$ completely depends on CD14 and TRIF (Yamamoto et al. 2003; Janova et al. 2016). The released IFN $\beta$ can bind to its receptor IFNAR, which initiates the canonical type I interferon signaling pathway. This involves activation of Jak1 and Tyk2 with subsequent recruitment of STAT1 and STAT2. The two transcription factors can then either form the ISGF3 complex together with IRF9 and bind to ISREs or bind to GAS, if they are not associated with IRF9 (Platanias 2005; Li et al. 1996). We could show that the canonical type I interferon signaling pathway, which is triggered in response to TLR4 activation, controls the production of TLR4-induced chemokines. While CXCL1 levels are negatively regulated, the release of CCL2 is positively regulated by this pathway. This provides evidence for a crosstalk between TLR and IFN signaling. The most important parts of the canonical type I interferon signaling pathway, namely IFNAR1 and IFNAR2, janus kinases, STAT1 and STAT2, were demonstrated to participate in an IFN $\beta$ mediated feedback regulation of CXCL1 and CCL2. In this regard, CCL2 expression could be either controlled by ISGF3 binding to its ISRE or STAT1/STAT2 heterodimer binding to its GAS (Valente et al. 1998; Graves et al. 1999). Within the CXCL1 promoter so far only a GAS was identified, which points towards a STAT1/STAT2-mediated regulation independent of IRF9 (Burke et al. 2014). Apart from these direct transcriptional effects, CCL2 and CXCL1 expression could be also indirectly regulated by another STAT1/STAT2-dependent gene, which controls chemokine expression. As a summary, the proposed mechanism is shown in Figure 5.1. 


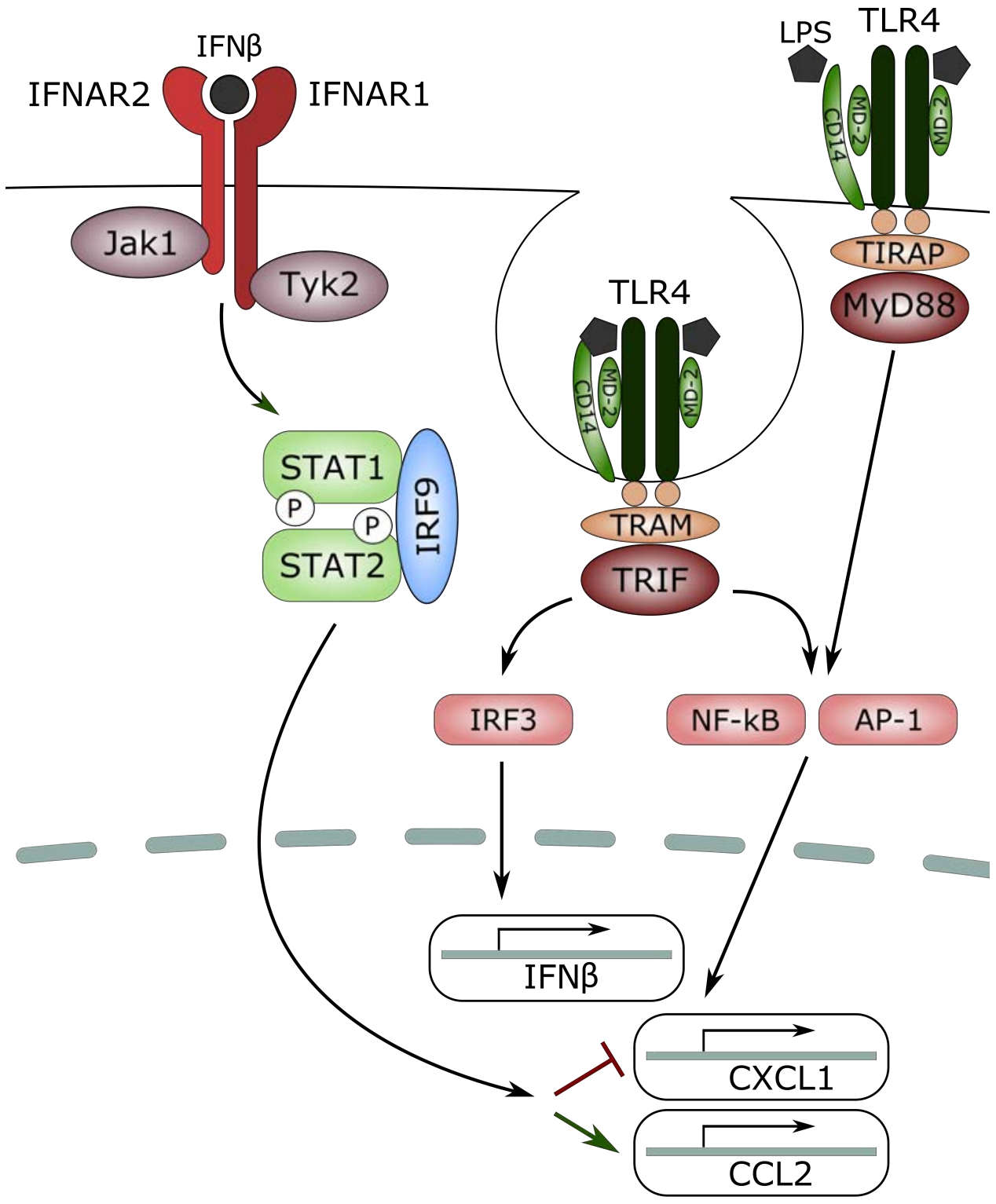

Figure 5.1: Proposed mechanism for the regulation of TLR4-induced chemokine production by the canonical type I interferon signaling pathway TLR4 gets activated by its ligand, here represented by LPS, and subsequently, the MyD88- and the TRIF-dependent signaling pathway are triggered. The TRIF-dependent signaling is enabled by CD14-induced endocytosis of the TLR4 complex. The activation of both signaling pathways induces the production of CXCL1 and CCL2 as well as IFN $\beta$. Thereby, the production of IFN $\beta$ completely depends on CD14 and TRIF. IFN $\beta$ can then bind to its receptor IFNAR, which initiates the canonical type I interferon signaling pathway. This involves activation of Jak1 and Tyk2 with subsequent recruitment of STAT1 and STAT2. Consequently, this pathway negatively regulates CXCL1 and positively regulates CCL2 production. Partially adapted from O'Neill et al. (2013) and Ivashkiv and Donlin (2014). 


\section{Summary and Conclusions}

Microglia are the tissue-resident macrophages of the brain parenchyma that serve as first and most important active immune defense within the CNS. They are equipped with a broad range of different receptor types in order to sense pathogens and homeostatic disturbances. Among these, TLRs that recognize highly conserved structures on foreign as well as host molecules play an important role. Depending on the type of challenge, activation of these receptors triggers different microglial functions and initiates an appropriate immune response.

Interferons are crucial cytokines for fighting viral, but also bacterial infections and are thus produced in response to several pathogens. Under different circumstances, microglia were already demonstrated to produce these immune mediators. We here investigated, which type I interferons can be produced upon activation of different TLRs. In response to viral infection as mimicked by the TLR3 ligand poly $\mathrm{I}: \mathrm{C}$, we showed that microglia are able to produce high amounts of $\operatorname{IFN} \beta$ and IFN $\alpha$. In contrast to that, only IFN $\beta$ was released in response to bacterial ligands and damage-related molecules that are sensed by TLR4. Surprisingly, bacterial ligands of TLR2 completely failed to induce any type I interferon. However, these differences in interferon production correlated with the differential involvement of the major TLR signaling routes. While TLR3 signals via the TRIF-dependent pathway and TLR2 uses the MyD88-dependent pathway, TLR4 activates both signaling cascades. We showed that production of IFN $\beta$ exclusively depends on the presence of TRIF and accordingly, only TLR3 and TLR4 that activate this signaling adaptor protein triggered IFN $\beta$. Within the TLR4 system, IFN $\beta$ release further depended on the TLR4 co-receptor CD14, which enables access of the receptor complex to TRIF-dependent signaling and therefore allows IFN $\beta$ production.

In addition to their important functions in host defense against viral and bacterial infections, various immunomodulatory effects of type I interferons have been identified. It was previously shown that microglial chemokine production upon activation of different TLRs can be regulated by IFN $\beta$. Here, we propose a mechanism how this regulation is achieved. We demonstrated that microglial CXCL1 and CCL2 release in response to TLR4, TLR2 and TLR3 are controlled by the type I interferon receptor. While CCL2 was positively regulated by IFNAR, the production of CXCL1 was under negative control of this receptor. An effect similar to that in microglia was observed in BMDM, while $\mathrm{pM} \Phi$ reacted partially different. Accordingly, IFNAR-dependent regulation of chemokine production is not restricted to the CNS, but still limited to specific immunological niches.

Within the TLR4 system, the regulatory effect of IFN $\beta$ involved all important parts of the canonical type I interferon signaling pathway. We proved that both type I interferon receptor subunits, IFNAR1 and IFNAR2, cooperatively regulate CXCL1 and CCL2 production. This regulation was mediated by janus kinases and further utilized the transcription factors STAT1 and STAT2. Within the TLR2 system, only IFNAR1 and IFNAR2 participated in the regulation of CXCL1 production by a signaling cascade that could not be further identified. This reveals an individual organization of responses to different TLRs 
under the control of distinct type I interferon signaling pathways.

Apart from identification of these regulatory mechanisms, we also demonstrated their relevance during CNS infection. In a model of gram-negative bacterial meningitis, type I interferon signaling controlled immune cell infiltration and thus, could shape disease outcome. In the absence of IFNAR1, excessive amounts of neutrophils were present in the brain of infected animals, which could lead to further damage of the tissue. In contrast to that, neutrophil infiltration in models of gram-positive bacterial meningitis and the autoimmune disease NMO were independent of IFNAR1. This highlights the specificity of type I interferon involvement in different diseases of the CNS.

Overall, this thesis shows that type I interferons are important regulators of microglial responses to CNS infection and damage. Upon recognition of harmful threats by different TLRs, microglia are potent producers of several type I interferons. At the same time, they are targeted by these immunomodulatory molecules in order to regulate the outcome of their immune responses, including chemokine production and subsequent recruitment of peripheral immune cells. The regulatory effect on different TLR systems is mediated by individual signaling pathways, which indicates a heterogeneous organization of microglial responses and shows the complexity of interferon actions. This understanding of distinct mechanisms by which type I interferons exert their functions could establish a basis for further unraveling the complex role of interferons in different CNS diseases. 



\section{Bibliography}

Ajami, B., J. L. Bennett, C. Krieger, W. Tetzlaff, and F. M. V. Rossi (2007). "Local Self-Renewal Can Sustain CNS Microglia Maintenance and Function throughout Adult Life". In: Nat. Neurosci. 10.12, pp. 1538-1543.

Akira, S., S. Uematsu, and O. Takeuchi (2006). "Pathogen Recognition and Innate Immunity". In: Cell 124.4, pp. 783-801.

Alexopoulou, L., A. C. Holt, R. Medzhitov, and R. A. Flavell (2001). "Recognition of Double-Stranded RNA and Activation of NF-kappaB by Toll-like Receptor 3". In: Nature 413.6857, pp. 732-738.

Aloisi, F. (2001). "Immune Function of Microglia". In: Glia 36.2, pp. 165-179.

Anderson, K. V., G. Jürgens, and C. Nüsslein-Volhard (1985). "Establishment of Dorsal-Ventral Polarity in the Drosophila Embryo: Genetic Studies on the Role of the Toll Gene Product". In: Cell 42.3, pp. 779-789.

Asavapanumas, N., J. Ratelade, and A. S. Verkman (2014). "Unique Neuromyelitis Optica Pathology Produced in Naïve Rats by Intracerebral Administration of NMO-IgG". In: Acta Neuropathol. 127.4, pp. 539-551.

Asea, A., S. K. Kraeft, E. A. Kurt-Jones, M. A. Stevenson, L. B. Chen, R. W. Finberg, G. C. Koo, and S. K. Calderwood (2000). "HSP70 Stimulates Cytokine Production through a CD14-Dependant Pathway, Demonstrating Its Dual Role as a Chaperone and Cytokine". In: Nat. Med. 6.4, pp. 435-442.

Atmaca, H. T., O. Kul, E. Karakuş, O. S. Terzi, S. Canpolat, and T. Anteplioğlu (2014). "Astrocytes, Microglia/Macrophages, and Neurons Expressing Toll-like Receptor 11 Contribute to Innate Immunity against Encephalitic Toxoplasma Gondii Infection". In: Neuroscience 269, pp. 184-191.

Auerbuch, V., D. G. Brockstedt, N. Meyer-Morse, M. O'Riordan, and D. A. Portnoy (2004). "Mice Lacking the Type I Interferon Receptor Are Resistant to Listeria Monocytogenes". In: J. Exp. Med. 200.4, pp. 527-533.

Auffray, C., D. Fogg, M. Garfa, G. Elain, O. Join-Lambert, S. Kayal, S. Sarnacki, A. Cumano, G. Lauvau, and F. Geissmann (2007). "Monitoring of Blood Vessels and Tissues by a Population of Monocytes with Patrolling Behavior". In: Science 317.5838, pp. 666-670.

Banati, R. B., J. Gehrmann, P. Schubert, and G. W. Kreutzberg (1993). "Cytotoxicity of Microglia". In: Glia 7.1, pp. 111-118.

Barbalat, R., L. Lau, R. M. Locksley, and G. M. Barton (2009). "Toll-like Receptor 2 on Inflammatory Monocytes Induces Type I Interferon in Response to Viral but Not Bacterial Ligands". In: Nat. Immunol. 10.11, pp. 1200-1207.

Barichello, T., J. S. Generoso, L. R. Simões, J. A. Goularte, F. Petronilho, P. Saigal, M. Badawy, and J. Quevedo (2016). "Role of Microglial Activation in the Pathophysiology of Bacterial Meningitis". In: Mol. Neurobiol. 53.3, pp. 1770-1781.

Bekisz, J., S. Baron, C. Balinsky, A. Morrow, and K. C. Zoon (2010). "Antiproliferative Properties of Type I and Type II Interferon". In: Pharmaceuticals (Basel) 3.4, pp. 994-1015. 
Bellingan, G. J., H. Caldwell, S. E. Howie, I. Dransfield, and C. Haslett (1996). "In Vivo Fate of the Inflammatory Macrophage during the Resolution of Inflammation: Inflammatory Macrophages Do Not Die Locally, but Emigrate to the Draining Lymph Nodes". In: J. Immunol. 157.6, pp. 2577-2585.

Beutler, B. and E. T. Rietschel (2003). "Innate Immune Sensing and Its Roots: The Story of Endotoxin". In: Nat. Rev. Immunol. 3.2, pp. 169-176.

Bhattacharya, S., W.-C. HuangFu, G. Dong, J. Qian, D. P. Baker, J. Karar, C. Koumenis, J. A. Diehl, and S. Y. Fuchs (2013). "Anti-Tumorigenic Effects of Type 1 Interferon Are Subdued by Integrated Stress Responses". In: Oncogene 32.36, pp. 4214-4221.

Bhoj, V. G. and Z. J. Chen (2009). "Ubiquitylation in Innate and Adaptive Immunity". In: Nature 458.7237, pp. 430-437.

Bowman, C. C., A. Rasley, S. L. Tranguch, and I. Marriott (2003). "Cultured Astrocytes Express Toll-like Receptors for Bacterial Products". In: Glia 43.3, pp. 281-291.

Boyle, D. L., K. Soma, J. Hodge, A. Kavanaugh, D. Mandel, P. Mease, R. Shurmur, A. K. Singhal, N. Wei, S. Rosengren, I. Kaplan, S. Krishnaswami, Z. Luo, J. Bradley, and G. S. Firestein (2015). "The JAK Inhibitor Tofacitinib Suppresses Synovial JAK1-STAT Signalling in Rheumatoid Arthritis". In: Ann. Rheum. Dis. 74.6, pp. 1311-1316.

Brandt, K. J., C. Fickentscher, E. K. O. Kruithof, and P. de Moerloose (2013). "TLR2 Ligands Induce NF-kappaB Activation from Endosomal Compartments of Human Monocytes". In: PLoS ONE 8.12, e80743.

Brinkmann, V., U. Reichard, C. Goosmann, B. Fauler, Y. Uhlemann, D. S. Weiss, Y. Weinrauch, and A. Zychlinsky (2004). "Neutrophil Extracellular Traps Kill Bacteria". In: Science 303.5663, pp. 15321535.

Brubaker, S. W., K. S. Bonham, I. Zanoni, and J. C. Kagan (2015). "Innate Immune Pattern Recognition: A Cell Biological Perspective". In: Annu. Rev. Immunol. 33, pp. 257-290.

Bsibsi, M., R. Ravid, D. Gveric, and J. M. van Noort (2002). "Broad Expression of Toll-like Receptors in the Human Central Nervous System". In: J. Neuropathol. Exp. Neurol. 61.11, pp. 1013-1021.

Burdon, P. C. E., C. Martin, and S. M. Rankin (2008). "Migration across the Sinusoidal Endothelium Regulates Neutrophil Mobilization in Response to ELR + CXC Chemokines". In: Br. J. Haematol. 142.1, pp. 100-108.

Burke, S. J., D. Lu, T. E. Sparer, T. Masi, M. R. Goff, M. D. Karlstad, and J. J. Collier (2014). "NF-kB and STAT1 Control CXCL1 and CXCL2 Gene Transcription”. In: Am. J. Physiol. Endocrinol. Metab. 306.2, E131-149.

Buss, C., B. Opitz, A. C. Hocke, J. Lippmann, V. van Laak, S. Hippenstiel, M. Krüll, N. Suttorp, and J. Eitel (2010). "Essential Role of Mitochondrial Antiviral Signaling, IFN Regulatory Factor (IRF)3, and IRF7 in Chlamydophila Pneumoniae-Mediated IFN-Beta Response and Control of Bacterial Replication in Human Endothelial Cells". In: J. Immunol. 184.6, pp. 3072-3078.

Butovsky, O., G. Landa, G. Kunis, Y. Ziv, H. Avidan, N. Greenberg, A. Schwartz, I. Smirnov, A. Pollack, S. Jung, and M. Schwartz (2006). "Induction and Blockage of Oligodendrogenesis by Differently Activated Microglia in an Animal Model of Multiple Sclerosis". In: J. Clin. Invest. 116.4, pp. 905-915.

Campbell, I. L., T. Krucker, S. Steffensen, Y. Akwa, H. C. Powell, T. Lane, D. J. Carr, L. H. Gold, S. J. Henriksen, and G. R. Siggins (1999). "Structural and Functional Neuropathology in Transgenic Mice with CNS Expression of IFN-Alpha”. In: Brain Res. 835.1, pp. 46-61.

Carpentier, P. A., W. S. Begolka, J. K. Olson, A. Elhofy, W. J. Karpus, and S. D. Miller (2005). "Differential Activation of Astrocytes by Innate and Adaptive Immune Stimuli". In: Glia 49.3, pp. 360-374.

Carty, M., R. Goodbody, M. Schröder, J. Stack, P. N. Moynagh, and A. G. Bowie (2006). "The Human Adaptor SARM Negatively Regulates Adaptor Protein TRIF-Dependent Toll-like Receptor Signaling". In: Nat. Immunol. 7.10, pp. 1074-1081. 
Caso, J. R., J. M. Pradillo, O. Hurtado, P. Lorenzo, M. A. Moro, and I. Lizasoain (2007). "Toll-like Receptor 4 Is Involved in Brain Damage and Inflammation after Experimental Stroke". In: Circulation 115.12, pp. 1599-1608.

Castaño, A., A. J. Herrera, J. Cano, and A. Machado (1998). "Lipopolysaccharide Intranigral Injection Induces Inflammatory Reaction and Damage in Nigrostriatal Dopaminergic System". In: J. Neurochem. 70.4, pp. 1584-1592.

Chen, G. Y. and G. Nuñez (2010). "Sterile Inflammation: Sensing and Reacting to Damage". In: Nat. Rev. Immunol. 10.12, pp. 826-837.

Chen, K., P. Iribarren, J. Hu, J. Chen, W. Gong, E. H. Cho, S. Lockett, N. M. Dunlop, and J. M. Wang (2006). "Activation of Toll-like Receptor 2 on Microglia Promotes Cell Uptake of Alzheimer DiseaseAssociated Amyloid Beta Peptide". In: J. Biol. Chem. 281.6, pp. 3651-3659.

Chen, M., A. Cheng, F. Candotti, Y. J. Zhou, A. Hymel, A. Fasth, L. D. Notarangelo, and J. J. O'Shea (2000). "Complex Effects of Naturally Occurring Mutations in the JAK3 Pseudokinase Domain: Evidence for Interactions between the Kinase and Pseudokinase Domains". In: Mol. Cell. Biol. 20.3, pp. 947-956.

Chen, S.-K., P. Tvrdik, E. Peden, S. Cho, S. Wu, G. Spangrude, and M. R. Capecchi (2010). "Hematopoietic Origin of Pathological Grooming in Hoxb8 Mutant Mice". In: Cell 141.5, pp. 775-785.

Cheon, H., E. G. Holvey-Bates, J. W. Schoggins, S. Forster, P. Hertzog, N. Imanaka, C. M. Rice, M. W. Jackson, D. J. Junk, and G. R. Stark (2013). "IFN-Beta-Dependent Increases in STAT1, STAT2, and IRF9 Mediate Resistance to Viruses and DNA Damage". In: EMBO J. 32.20, pp. 2751-2763.

Chiu, Y.-H., J. B. Macmillan, and Z. J. Chen (2009). "RNA Polymerase III Detects Cytosolic DNA and Induces Type I Interferons through the RIG-I Pathway". In: Cell 138.3, pp. 576-591.

Cohen, B., D. Novick, S. Barak, and M. Rubinstein (1995). "Ligand-Induced Association of the Type I Interferon Receptor Components". In: Mol. Cell. Biol. 15.8, pp. 4208-4214.

Colamonici, O. and P. Domanski (1993). "Identification of a Novel Subunit of the Type I Interferon Receptor Localized to Human Chromosome 21". In: J. Biol. Chem. 268.15, pp. 10895-10899.

Colamonici, O., H. Yan, P. Domanski, R. Handa, D. Smalley, J. Mullersman, M. Witte, K. Krishnan, and J. Krolewski (1994). "Direct Binding to and Tyrosine Phosphorylation of the Alpha Subunit of the Type I Interferon Receptor by p135tyk2 Tyrosine Kinase”. In: Mol. Cell. Biol. 14.12, pp. 8133-8142.

Costello, D. A. and M. A. Lynch (2013). "Toll-like Receptor 3 Activation Modulates Hippocampal Network Excitability, via Glial Production of Interferon-Beta”. In: Hippocampus 23.8, pp. 696-707.

Croker, B. A., H. Kiu, and S. E. Nicholson (2008). "SOCS Regulation of the JAK/STAT Signalling Pathway”. In: Semin. Cell Dev. Biol. 19.4, pp. 414-422.

Crow, Y. J. and J. Rehwinkel (2009). "Aicardi-Goutieres Syndrome and Related Phenotypes: Linking Nucleic Acid Metabolism with Autoimmunity”. In: Hum. Mol. Genet. 18 (R2), R130-136.

Darnell, J. E., I. M. Kerr, and G. R. Stark (1994). "Jak-STAT Pathways and Transcriptional Activation in Response to IFNs and Other Extracellular Signaling Proteins". In: Science 264.5164, pp. 1415-1421.

David-Watine, B., A. Israël, and P. Kourilsky (1990). "The Regulation and Expression of MHC Class I Genes". In: Immunol. Today 11.8, pp. 286-292.

Davies, L. C., S. J. Jenkins, J. E. Allen, and P. R. Taylor (2013). "Tissue-Resident Macrophages". In: Nat. Immunol. 14.10, pp. 986-995.

Davies, L. C. and P. R. Taylor (2015). "Tissue-Resident Macrophages: Then and Now". In: Immunology 144.4, pp. 541-548.

De la Maza, L. M., E. M. Peterson, J. M. Goebel, C. W. Fennie, and C. W. Czarniecki (1985). "InterferonInduced Inhibition of Chlamydia Trachomatis: Dissociation from Antiviral and Antiproliferative Effects". In: Infect. Immun. 47.3, pp. 719-722. 
De Miranda, J., K. Yaddanapudi, M. Hornig, and W. I. Lipkin (2009). "Astrocytes Recognize Intracellular Polyinosinic-Polycytidylic Acid via MDA-5”. In: FASEB J. 23.4, pp. 1064-1071.

De Weerd, N. A., S. A. Samarajiwa, and P. J. Hertzog (2007). "Type I Interferon Receptors: Biochemistry and Biological Functions". In: J. Biol. Chem. 282.28, pp. 20053-20057.

De Weerd, N. A., J. P. Vivian, T. K. Nguyen, N. E. Mangan, J. A. Gould, S.-J. Braniff, L. Zaker-Tabrizi, K. Y. Fung, S. C. Forster, T. Beddoe, H. H. Reid, J. Rossjohn, and P. J. Hertzog (2013). "Structural Basis of a Unique Interferon-Beta Signaling Axis Mediated via the Receptor IFNAR1". In: Nat. Immunol. 14.9, pp. 901-907.

Decker, T., M. Müller, and S. Stockinger (2005). "The Yin and Yang of Type I Interferon Activity in Bacterial Infection". In: Nat. Rev. Immunol. 5.9, pp. 675-687.

Del Río-Hortega, P. (1919). "El "tercer Elemento" de Los Centros Nerviosos. I. La Microglía En Estado Normal. II. Intervención de La Microglía En Los Procesos Patológicos (Células En Bastoncito Y Cuerpos Gránulo-Adiposos). III. Naturaleza Probable de La Microglía". In: Boletín de la Sociedad Espanola de Biología 8, pp. 69-120.

Delhaye, S., S. Paul, G. Blakqori, M. Minet, F. Weber, P. Staeheli, and T. Michiels (2006). "Neurons Produce Type I Interferon during Viral Encephalitis". In: Proc. Natl. Acad. Sci. U.S.A. 103.20, pp. 78357840.

Dietrich, N., S. Lienenklaus, S. Weiss, and N. O. Gekara (2010). "Murine Toll-like Receptor 2 Activation Induces Type I Interferon Responses from Endolysosomal Compartments". In: PLOS ONE 5.4, e10250.

Domanski, P., E. Fish, O. W. Nadeau, M. Witte, L. C. Platanias, H. Yan, J. Krolewski, P. Pitha, and O. R. Colamonici (1997). "A Region of the Beta Subunit of the Interferon Alpha Receptor Different from Box 1 Interacts with Jak1 and Is Sufficient to Activate the Jak-Stat Pathway and Induce an Antiviral State". In: J. Biol. Chem. 272.42, pp. 26388-26393.

Echchannaoui, H., K. Frei, C. Schnell, S. L. Leib, W. Zimmerli, and R. Landmann (2002). "Toll-like Receptor 2-Deficient Mice Are Highly Susceptible to Streptococcus Pneumoniae Meningitis because of Reduced Bacterial Clearing and Enhanced Inflammation". In: J. Infect. Dis. 186.6, pp. 798-806.

Elmore, M. R. P., A. R. Najafi, M. A. Koike, N. N. Dagher, E. E. Spangenberg, R. A. Rice, M. Kitazawa, B. Matusow, H. Nguyen, B. L. West, and K. N. Green (2014). "Colony-Stimulating Factor 1 Receptor Signaling Is Necessary for Microglia Viability, Unmasking a Microglia Progenitor Cell in the Adult Brain". In: Neuron 82.2, pp. 380-397.

Epelman, S., K. J. Lavine, and G. J. Randolph (2014). "Origin and Functions of Tissue Macrophages". In: Immunity 41.1, pp. 21-35.

Fantuzzi, L., I. Canini, F. Belardelli, and S. Gessani (2001). "IFN-Beta Stimulates the Production of BetaChemokines in Human Peripheral Blood Monocytes. Importance of Macrophage Differentiation". In: Eur. Cytokine Netw. 12.4, pp. 597-603.

Farrar, J. D., J. D. Smith, T. L. Murphy, and K. M. Murphy (2000). "Recruitment of Stat4 to the Human Interferon-Alpha/Beta Receptor Requires Activated Stat2". In: J. Biol. Chem. 275.4, pp. 2693-2697.

Fasler-Kan, E., A. Pansky, M. Wiederkehr, M. Battegay, and M. H. Heim (1998). "Interferon-Alpha Activates Signal Transducers and Activators of Transcription 5 and 6 in Daudi Cells". In: Eur. J. Biochem. 254.3, pp. 514-519.

Fernandez, H. N., P. M. Henson, A. Otani, and T. E. Hugli (1978). "Chemotactic Response to Human $\mathrm{C} 3 \mathrm{a}$ and $\mathrm{C} 5 \mathrm{a}$ Anaphylatoxins. I. Evaluation of $\mathrm{C} 3 \mathrm{a}$ and C5a Leukotaxis in Vitro and under Stimulated in Vivo Conditions". In: J. Immunol. 120.1, pp. 109-115.

Fitzgerald, K. A., S. M. McWhirter, K. L. Faia, D. C. Rowe, E. Latz, D. T. Golenbock, A. J. Coyle, S.-M. Liao, and T. Maniatis (2003). "IKKepsilon and TBK1 Are Essential Components of the IRF3 Signaling Pathway". In: Nat. Immunol. 4.5, pp. 491-496. 
Fitzgerald-Bocarsly, P. and D. Feng (2007). "The Role of Type I Interferon Production by Dendritic Cells in Host Defense". In: Biochimie 89 (6-7), pp. 843-855.

Fitzner, D., M. Schnaars, D. van Rossum, G. Krishnamoorthy, P. Dibaj, M. Bakhti, T. Regen, U.-K. Hanisch, and M. Simons (2011). "Selective Transfer of Exosomes from Oligodendrocytes to Microglia by Macropinocytosis". In: J. Cell. Sci. 124 (Pt 3), pp. 447-458.

Fu, X. Y., C. Schindler, T. Improta, R. Aebersold, and J. E. Darnell (1992). "The Proteins of ISGF3, the Interferon Alpha-Induced Transcriptional Activator, Define a Gene Family Involved in Signal Transduction". In: Proc. Natl. Acad. Sci. U.S.A. 89.16, pp. 7840-7843.

Furr, S. R., V. S. Chauhan, M. J. Moerdyk-Schauwecker, and I. Marriott (2011). "A Role for DNADependent Activator of Interferon Regulatory Factor in the Recognition of Herpes Simplex Virus Type 1 by Glial Cells". In: J Neuroinflammation 8, p. 99.

Furr, S. R., V. S. Chauhan, D. Sterka, V. Grdzelishvili, and I. Marriott (2008). "Characterization of Retinoic Acid-Inducible Gene-I Expression in Primary Murine Glia Following Exposure to Vesicular Stomatitis Virus". In: J. Neurovirol. 14.6, pp. 503-513.

Furr, S. R., M. Moerdyk-Schauwecker, V. Z. Grdzelishvili, and I. Marriott (2010). "RIG-I Mediates Nonsegmented Negative-Sense RNA Virus-Induced Inflammatory Immune Responses of Primary Human Astrocytes". In: Glia 58.13, pp. 1620-1629.

Galea, I., I. Bechmann, and V. H. Perry (2007). "What Is Immune Privilege (Not)?" In: Trends Immunol. 28.1, pp. 12-18.

Galligan, C. L., L. M. Pennell, T. T. Murooka, E. Baig, B. Majchrzak-Kita, R. Rahbar, and E. N. Fish (2010). "Interferon-Beta Is a Key Regulator of Proinflammatory Events in Experimental Autoimmune Encephalomyelitis". In: Mult. Scler. 16.12, pp. 1458-1473.

Gao, H.-M. and J.-S. Hong (2008). "Why Neurodegenerative Diseases Are Progressive: Uncontrolled Inflammation Drives Disease Progression". In: Trends Immunol. 29.8, pp. 357-365.

Garden, G. A. and T. Möller (2006). "Microglia Biology in Health and Disease". In: J Neuroimmune Pharmacol 1.2, pp. 127-137.

GBD 2013 Mortality and Causes of Death Collaborators (2015). "Global, Regional, and National AgeSex Specific All-Cause and Cause-Specific Mortality for 240 Causes of Death, 1990-2013: A Systematic Analysis for the Global Burden of Disease Study 2013". In: Lancet 385.9963, pp. 117-171.

Geissmann, F., S. Jung, and D. R. Littman (2003). "Blood Monocytes Consist of Two Principal Subsets with Distinct Migratory Properties". In: Immunity 19.1, pp. 71-82.

Gerber, J., G. Raivich, A. Wellmer, C. Noeske, T. Kunst, A. Werner, W. Brück, and R. Nau (2001). "A Mouse Model of Streptococcus Pneumoniae Meningitis Mimicking Several Features of Human Disease". In: Acta Neuropathol. 101.5, pp. 499-508.

Gerber, J. and R. Nau (2010). "Mechanisms of Injury in Bacterial Meningitis". In: Curr. Opin. Neurol. 23.3, pp. 312-318.

Gesuete, R., A. E. B. Packard, K. B. Vartanian, V. K. Conrad, S. L. Stevens, F. R. Bahjat, T. Yang, and M. P. Stenzel-Poore (2012). "Poly-ICLC Preconditioning Protects the Blood-Brain Barrier against Ischemic Injury in Vitro through Type I Interferon Signaling”. In: J. Neurochem. 123 Suppl 2, pp. 7585.

Ghoreschi, K., A. Laurence, and J. J. O'Shea (2009). “Janus Kinases in Immune Cell Signaling”. In: Immunol. Rev. 228.1, pp. 273-287.

Ginhoux, F., M. Greter, M. Leboeuf, S. Nandi, P. See, S. Gokhan, M. F. Mehler, S. J. Conway, L. G. Ng, E. R. Stanley, I. M. Samokhvalov, and M. Merad (2010). "Fate Mapping Analysis Reveals That Adult Microglia Derive from Primitive Macrophages". In: Science 330.6005, pp. 841-845. 
Girardin, S. E., I. G. Boneca, J. Viala, M. Chamaillard, A. Labigne, G. Thomas, D. J. Philpott, and P. J. Sansonetti (2003). "Nod2 Is a General Sensor of Peptidoglycan through Muramyl Dipeptide (MDP) Detection". In: J. Biol. Chem. 278.11, pp. 8869-8872.

Girault, J. A., G. Labesse, J. P. Mornon, and I. Callebaut (1998). "Janus Kinases and Focal Adhesion Kinases Play in the 4.1 Band: A Superfamily of Band 4.1 Domains Important for Cell Structure and Signal Transduction". In: Mol. Med. 4.12, pp. 751-769.

Glezer, I., A. Lapointe, and S. Rivest (2006). "Innate Immunity Triggers Oligodendrocyte Progenitor Reactivity and Confines Damages to Brain Injuries". In: FASEB J. 20.6, pp. 750-752.

Global Burden of Disease Study 2013 Collaborators (2015). "Global, Regional, and National Incidence, Prevalence, and Years Lived with Disability for 301 Acute and Chronic Diseases and Injuries in 188 Countries, 1990-2013: A Systematic Analysis for the Global Burden of Disease Study 2013". In: Lancet 386.9995, pp. 743-800.

Goh, K. C., S. J. Haque, and B. R. Williams (1999). "p38 MAP Kinase Is Required for STAT1 Serine Phosphorylation and Transcriptional Activation Induced by Interferons". In: EMBO J. 18.20, pp. 56015608.

González-Navajas, J. M., J. Lee, M. David, and E. Raz (2012). "Immunomodulatory Functions of Type I Interferons". In: Nat. Rev. Immunol. 12.2, pp. 125-135.

Gough, D. J., N. L. Messina, C. J. P. Clarke, R. W. Johnstone, and D. E. Levy (2012). "Constitutive Type I Interferon Modulates Homeostatic Balance through Tonic Signaling”. In: Immunity 36.2, pp. 166-174.

Grafi, G., I. Sela, and G. Galili (1993). "Translational Regulation of Human Beta Interferon mRNA: Association of the 3' AU-Rich Sequence with the poly(A) Tail Reduces Translation Efficiency in Vitro". In: Mol. Cell. Biol. 13.6, pp. 3487-3493.

Graves, D. T., Y. Jiang, and A. J. Valente (1999). "The Expression of Monocyte Chemoattractant Protein1 and Other Chemokines by Osteoblasts". In: Front. Biosci. 4, pp. D571-580.

Hamilton, J. A., G. A. Whitty, I. Kola, and P. J. Hertzog (1996). "Endogenous IFN-Alpha Beta Suppresses Colony-Stimulating Factor (CSF)-1-Stimulated Macrophage DNA Synthesis and Mediates Inhibitory Effects of Lipopolysaccharide and TNF-Alpha". In: J. Immunol. 156.7, pp. 2553-2557.

Hanisch, U.-K. (2002). "Microglia as a Source and Target of Cytokines". In: Glia 40.2, pp. 140-155.

Hanisch, U.-K., T. V. Johnson, and J. Kipnis (2008). "Toll-like Receptors: Roles in Neuroprotection?" In: Trends Neurosci. 31.4, pp. 176-182.

Hanisch, U.-K. and H. Kettenmann (2007). "Microglia: Active Sensor and Versatile Effector Cells in the Normal and Pathologic Brain". In: Nat. Neurosci. 10.11, pp. 1387-1394.

Hardy, M. P., C. M. Owczarek, L. S. Jermiin, M. Ejdebäck, and P. J. Hertzog (2004). "Characterization of the Type I Interferon Locus and Identification of Novel Genes". In: Genomics 84.2, pp. 331-345.

Hauss-Wegrzyniak, B., P. Dobrzanski, J. D. Stoehr, and G. L. Wenk (1998). "Chronic Neuroinflammation in Rats Reproduces Components of the Neurobiology of Alzheimer's Disease". In: Brain Res. 780.2, pp. 294-303.

Hayes, G. M., M. N. Woodroofe, and M. L. Cuzner (1987). "Microglia Are the Major Cell Type Expressing MHC Class II in Human White Matter". In: J. Neurol. Sci. 80.1, pp. 25-37.

Heim, M. H., I. M. Kerr, G. R. Stark, and J. E. Darnell (1995). "Contribution of STAT SH2 Groups to Specific Interferon Signaling by the Jak-STAT Pathway”. In: Science 267.5202, pp. 1347-1349.

Heine, A., S. A. E. Held, S. N. Daecke, S. Wallner, S. P. Yajnanarayana, C. Kurts, D. Wolf, and P. Brossart (2013). "The JAK-Inhibitor Ruxolitinib Impairs Dendritic Cell Function in Vitro and in Vivo". In: Blood 122.7, pp. 1192-1202.

Herges, K., B. A. de Jong, I. Kolkowitz, C. Dunn, G. Mandelbaum, R. M. Ko, A. Maini, M. H. Han, J. Killestein, C. Polman, A. L. Goodyear, J. Dunn, L. Steinman, and R. C. Axtell (2012). "Protective 
Effect of an Elastase Inhibitor in a Neuromyelitis Optica-like Disease Driven by a Peptide of Myelin Oligodendroglial Glycoprotein". In: Mult. Scler. 18.4, pp. 398-408.

Hervas-Stubbs, S., J. L. Perez-Gracia, A. Rouzaut, M. F. Sanmamed, A. Le Bon, and I. Melero (2011).

"Direct Effects of Type I Interferons on Cells of the Immune System". In: Clin. Cancer Res. 17.9, pp. 2619-2627.

Hesselgesser, J. and R. Horuk (1999). "Chemokine and Chemokine Receptor Expression in the Central Nervous System". In: J. Neurovirol. 5.1, pp. 13-26.

Hoffmann, O., J. S. Braun, D. Becker, A. Halle, D. Freyer, E. Dagand, S. Lehnardt, and J. R. Weber (2007). "TLR2 Mediates Neuroinflammation and Neuronal Damage". In: J. Immunol. 178.10, pp. 6476-6481.

Honda, S., Y. Sasaki, K. Ohsawa, Y. Imai, Y. Nakamura, K. Inoue, and S. Kohsaka (2001). "Extracellular ATP or ADP Induce Chemotaxis of Cultured Microglia through Gi/O-Coupled P2Y Receptors". In: J. Neurosci. 21.6, pp. 1975-1982.

Hoshino, K., O. Takeuchi, T. Kawai, H. Sanjo, T. Ogawa, Y. Takeda, K. Takeda, and S. Akira (1999). "Cutting Edge: Toll-like Receptor 4 (TLR4)-Deficient Mice Are Hyporesponsive to Lipopolysaccharide: Evidence for TLR4 as the Lps Gene Product". In: J. Immunol. 162.7, pp. 3749-3752.

Huangfu, W.-C., J. Qian, C. Liu, J. Liu, A. E. Lokshin, D. P. Baker, H. Rui, and S. Y. Fuchs (2012). "Inflammatory Signaling Compromises Cell Responses to Interferon Alpha”. In: Oncogene 31.2, pp. 161172.

Hwang, S. Y., P. J. Hertzog, K. A. Holland, S. H. Sumarsono, M. J. Tymms, J. A. Hamilton, G. Whitty, I. Bertoncello, and I. Kola (1995). "A Null Mutation in the Gene Encoding a Type I Interferon Receptor Component Eliminates Antiproliferative and Antiviral Responses to Interferons Alpha and Beta and Alters Macrophage Responses". In: Proc. Natl. Acad. Sci. U.S.A. 92.24, pp. 11284-11288.

Imhof, B. A., S. Jemelin, R. Ballet, C. Vesin, M. Schapira, M. Karaca, and Y. Emre (2016). "CCN1/CYR61Mediated Meticulous Patrolling by Ly6Clow Monocytes Fuels Vascular Inflammation". In: Proc. Natl. Acad. Sci. U.S.A. 113.33, E4847-4856.

Isaacs, A. and J. Lindenmann (1957). "Virus Interference. I. The Interferon". In: Proc. R. Soc. Lond., B, Biol. Sci. 147.927, pp. 258-267.

Ishikawa, H., Z. Ma, and G. N. Barber (2009). "STING Regulates Intracellular DNA-Mediated, Type I Interferon-Dependent Innate Immunity". In: Nature 461.7265, pp. 788-792.

Ishizaki, M., R. Muromoto, T. Akimoto, Y. Ohshiro, M. Takahashi, Y. Sekine, H. Maeda, K. Shimoda, K. Oritani, and T. Matsuda (2011). "Tyk2 Deficiency Protects Joints against Destruction in Anti-Type II Collagen Antibody-Induced Arthritis in Mice". In: Int. Immunol. 23.9, pp. 575-582.

Ishizaki, M., R. Muromoto, T. Akimoto, Y. Sekine, S. Kon, M. Diwan, H. Maeda, S. Togi, K. Shimoda, K. Oritani, and T. Matsuda (2014). "Tyk2 Is a Therapeutic Target for Psoriasis-like Skin Inflammation". In: Int. Immunol. 26.5, pp. 257-267.

Ivashkiv, L. B. and L. T. Donlin (2014). "Regulation of Type I Interferon Responses". In: Nat. Rev. Immunol. 14.1, pp. 36-49.

Jack, C. S., N. Arbour, J. Manusow, V. Montgrain, M. Blain, E. McCrea, A. Shapiro, and J. P. Antel (2005). "TLR Signaling Tailors Innate Immune Responses in Human Microglia and Astrocytes". In: J. Immunol. 175.7, pp. 4320-4330.

Jackson, A. C., J. P. Rossiter, and M. Lafon (2006). "Expression of Toll-like Receptor 3 in the Human Cerebellar Cortex in Rabies, Herpes Simplex Encephalitis, and Other Neurological Diseases". In: J. Neurovirol. 12.3, pp. 229-234.

Jaks, E., M. Gavutis, G. Uzé, J. Martal, and J. Piehler (2007). "Differential Receptor Subunit Affinities of Type I Interferons Govern Differential Signal Activation”. In: J. Mol. Biol. 366.2, pp. 525-539. 
Janeway, C. A., P. Travers, M. Walport, and M. J. Shlomchik (2001). Immunobiology. 5th ed. New York: Garland Science.

Janova, H., C. Böttcher, I. R. Holtman, T. Regen, D. van Rossum, A. Götz, A.-S. Ernst, C. Fritsche,

U. Gertig, N. Saiepour, K. Gronke, C. Wrzos, S. Ribes, S. Rolfes, J. Weinstein, H. Ehrenreich, T.

Pukrop, J. Kopatz, C. Stadelmann, G. Salinas-Riester, M. S. Weber, M. Prinz, W. Brück, B. J. L. Eggen, H. W. G. M. Boddeke, J. Priller, and U.-K. Hanisch (2016). "CD14 Is a Key Organizer of Microglial Responses to CNS Infection and Injury”. In: Glia 64.4, pp. 635-649.

Jarius, S., F. Paul, D. Franciotta, K. Ruprecht, M. Ringelstein, R. Bergamaschi, P. Rommer, I. Kleiter, O. Stich, R. Reuss, S. Rauer, U. K. Zettl, K. P. Wandinger, A. Melms, O. Aktas, W. Kristoferitsch, and B. Wildemann (2011). "Cerebrospinal Fluid Findings in Aquaporin-4 Antibody Positive Neuromyelitis Optica: Results from 211 Lumbar Punctures". In: J. Neurol. Sci. 306 (1-2), pp. 82-90.

Jia, T., I. Leiner, G. Dorothee, K. Brandl, and E. G. Pamer (2009). "MyD88 and Type I Interferon Receptor-Mediated Chemokine Induction and Monocyte Recruitment during Listeria Monocytogenes Infection". In: J. Immunol. 183.2, pp. 1271-1278.

Jiang, Z., P. Georgel, X. Du, L. Shamel, S. Sovath, S. Mudd, M. Huber, C. Kalis, S. Keck, C. Galanos, M. Freudenberg, and B. Beutler (2005). "CD14 Is Required for MyD88-Independent LPS Signaling". In: Nat. Immunol. 6.6, pp. 565-570.

Jin, M. S., S. E. Kim, J. Y. Heo, M. E. Lee, H. M. Kim, S.-G. Paik, H. Lee, and J.-O. Lee (2007). "Crystal Structure of the TLR1-TLR2 Heterodimer Induced by Binding of a Tri-Acylated Lipopeptide". In: Cell 130.6, pp. 1071-1082.

Johnson, E. A., T. L. Dao, M. A. Guignet, C. E. Geddes, A. I. Koemeter-Cox, and R. K. Kan (2011). "Increased Expression of the Chemokines CXCL1 and MIP-1a by Resident Brain Cells Precedes Neutrophil Infiltration in the Brain Following Prolonged Soman-Induced Status Epilepticus in Rats". In: $J$ Neuroinflammation 8, p. 41.

Jung, D. Y., H. Lee, B.-Y. Jung, J. Ock, M.-S. Lee, W.-H. Lee, and K. Suk (2005). "TLR4, but Not TLR2, Signals Autoregulatory Apoptosis of Cultured Microglia: A Critical Role of IFN-Beta as a Decision Maker". In: J. Immunol. 174.10, pp. 6467-6476.

Kagan, J. C., T. Su, T. Horng, A. Chow, S. Akira, and R. Medzhitov (2008). "TRAM Couples Endocytosis of Toll-like Receptor 4 to the Induction of Interferon-Beta". In: Nat. Immunol. 9.4, pp. 361-368.

Kahl, L., J. Patel, M. Layton, M. Binks, K. Hicks, G. Leon, E. Hachulla, D. Machado, D. Staumont-Sallé, M. Dickson, L. Condreay, L. Schifano, S. Zamuner, R. F. van Vollenhoven, and JAK115919 Study Team (2016). "Safety, Tolerability, Efficacy and Pharmacodynamics of the Selective JAK1 Inhibitor GSK2586184 in Patients with Systemic Lupus Erythematosus". In: Lupus, [Epub ahead of print].

Kallfass, C., A. Ackerman, S. Lienenklaus, S. Weiss, B. Heimrich, and P. Staeheli (2012). "Visualizing Production of Beta Interferon by Astrocytes and Microglia in Brain of La Crosse Virus-Infected Mice". In: J. Virol. 86.20, pp. 11223-11230.

Kang, J. Y. and J.-O. Lee (2011). "Structural Biology of the Toll-like Receptor Family". In: Annu. Rev. Biochem. 80, pp. 917-941.

Kantari, C., M. Pederzoli-Ribeil, and V. Witko-Sarsat (2008). "The Role of Neutrophils and Monocytes in Innate Immunity". In: Contrib Microbiol 15, pp. 118-146.

Karaghiosoff, M., H. Neubauer, C. Lassnig, P. Kovarik, H. Schindler, H. Pircher, B. McCoy, C. Bogdan, T. Decker, G. Brem, K. Pfeffer, and M. Müller (2000). "Partial Impairment of Cytokine Responses in Tyk2-Deficient Mice". In: Immunity 13.4, pp. 549-560.

Karaghiosoff, M., R. Steinborn, P. Kovarik, G. Kriegshäuser, M. Baccarini, B. Donabauer, U. Reichart,

T. Kolbe, C. Bogdan, T. Leanderson, D. Levy, T. Decker, and M. Müller (2003). "Central Role for Type I Interferons and Tyk2 in Lipopolysaccharide-Induced Endotoxin Shock". In: Nat. Immunol. 4.5, pp. 471-477. 
Kaur, C., H. F. Too, and E. A. Ling (2004). "Phagocytosis of Escherichia Coli by Amoeboid Microglial Cells in the Developing Brain". In: Acta Neuropathol. 107.3, pp. 204-208.

Kawagoe, T., S. Sato, K. Matsushita, H. Kato, K. Matsui, Y. Kumagai, T. Saitoh, T. Kawai, O. Takeuchi, and S. Akira (2008). "Sequential Control of Toll-like Receptor-Dependent Responses by IRAK1 and IRAK2". In: Nat. Immunol. 9.6, pp. 684-691.

Kawai, T. and S. Akira (2010). "The Role of Pattern-Recognition Receptors in Innate Immunity: Update on Toll-like Receptors". In: Nat. Immunol. 11.5, pp. 373-384.

Kawai, T., S. Sato, K. J. Ishii, C. Coban, H. Hemmi, M. Yamamoto, K. Terai, M. Matsuda, J.-i. Inoue, S. Uematsu, O. Takeuchi, and S. Akira (2004). "Interferon-Alpha Induction through Toll-like Receptors Involves a Direct Interaction of IRF7 with MyD88 and TRAF6". In: Nat. Immunol. 5.10, pp. 1061-1068.

Kelly-Scumpia, K. M., P. O. Scumpia, M. J. Delano, J. S. Weinstein, A. G. Cuenca, J. L. Wynn, and L. L. Moldawer (2010). "Type I Interferon Signaling in Hematopoietic Cells Is Required for Survival in Mouse Polymicrobial Sepsis by Regulating CXCL10”. In: J. Exp. Med. 207.2, pp. 319-326.

Kettenmann, H., U.-K. Hanisch, M. Noda, and A. Verkhratsky (2011). "Physiology of Microglia". In: Physiol. Rev. 91.2, pp. 461-553.

Khorooshi, R. and T. Owens (2010). "Injury-Induced Type I IFN Signaling Regulates Inflammatory Responses in the Central Nervous System". In: J. Immunol. 185.2, pp. 1258-1264.

Khorooshi, R., A. Wlodarczyk, N. Asgari, and T. Owens (2013). "Neuromyelitis Optica-like Pathology Is Dependent on Type I Interferon Response". In: Exp. Neurol. 247, pp. 744-747.

Kierdorf, K., D. Erny, T. Goldmann, V. Sander, C. Schulz, E. G. Perdiguero, P. Wieghofer, A. Heinrich, P. Riemke, C. Hölscher, D. N. Müller, B. Luckow, T. Brocker, K. Debowski, G. Fritz, G. Opdenakker, A. Diefenbach, K. Biber, M. Heikenwalder, F. Geissmann, F. Rosenbauer, and M. Prinz (2013). "Microglia Emerge from Erythromyeloid Precursors via Pu.1- and Irf8-Dependent Pathways". In: Nat. Neurosci. 16.3, pp. 273-280.

Kigerl, K. A., W. Lai, S. Rivest, R. P. Hart, A. R. Satoskar, and P. G. Popovich (2007). "Toll-like Receptor (TLR)-2 and TLR-4 Regulate Inflammation, Gliosis, and Myelin Sparing after Spinal Cord Injury". In: J. Neurochem. 102.1, pp. 37-50.

Kim, D., M. A. Kim, I.-H. Cho, M. S. Kim, S. Lee, E.-K. Jo, S.-Y. Choi, K. Park, J. S. Kim, S. Akira, H. S. $\mathrm{Na}, \mathrm{S}$. B. Oh, and S. J. Lee (2007). "A Critical Role of Toll-like Receptor 2 in Nerve Injury-Induced Spinal Cord Glial Cell Activation and Pain Hypersensitivity". In: J. Biol. Chem. 282.20, pp. 1497514983.

Kim, S.-H., W. Kim, X. F. Li, I.-J. Jung, and H. J. Kim (2012). "Does Interferon Beta Treatment Exacerbate Neuromyelitis Optica Spectrum Disorder?" In: Mult. Scler. 18.10, pp. 1480-1483.

Kim, S., S. Y. Kim, J. P. Pribis, M. Lotze, K. P. Mollen, R. Shapiro, P. Loughran, M. J. Scott, and T. R. Billiar (2013). "Signaling of High Mobility Group Box 1 (HMGB1) through Toll-like Receptor 4 in Macrophages Requires CD14". In: Mol. Med. 19, pp. 88-98.

Kimbrough, D. J., K. Fujihara, A. Jacob, M. A. Lana-Peixoto, M. I. Leite, M. Levy, R. Marignier, I. Nakashima, J. Palace, J. de Seze, O. Stuve, S. N. Tenembaum, A. Traboulsee, E. Waubant, B. G. Weinshenker, D. M. Wingerchuk, and GJCF-CC\&BR (2012). "Treatment of Neuromyelitis Optica: Review and Recommendations". In: Mult Scler Relat Disord 1.4, pp. 180-187.

Kirou, K. A., C. Lee, S. George, K. Louca, M. G. E. Peterson, and M. K. Crow (2005). "Activation of the Interferon-Alpha Pathway Identifies a Subgroup of Systemic Lupus Erythematosus Patients with Distinct Serologic Features and Active Disease". In: Arthritis Rheum. 52.5, pp. 1491-1503.

Kobayashi, K., L. D. Hernandez, J. E. Galán, C. A. Janeway, R. Medzhitov, and R. A. Flavell (2002). “IRAK-M Is a Negative Regulator of Toll-like Receptor Signaling”. In: Cell 110.2, pp. 191-202. 
Koblansky, A. A., D. Jankovic, H. Oh, S. Hieny, W. Sungnak, R. Mathur, M. S. Hayden, S. Akira, A. Sher, and S. Ghosh (2013). "Recognition of Profilin by Toll-like Receptor 12 Is Critical for Host Resistance to Toxoplasma Gondii". In: Immunity 38.1, pp. 119-130.

Koedel, U., B. Angele, T. Rupprecht, H. Wagner, A. Roggenkamp, H.-W. Pfister, and C. J. Kirschning (2003). "Toll-like Receptor 2 Participates in Mediation of Immune Response in Experimental Pneumococcal Meningitis". In: J. Immunol. 170.1, pp. 438-444.

Kolaczkowska, E. and P. Kubes (2013). "Neutrophil Recruitment and Function in Health and Inflammation". In: Nat. Rev. Immunol. 13.3, pp. 159-175.

Kono, H. and K. L. Rock (2008). "How Dying Cells Alert the Immune System to Danger". In: Nat. Rev. Immunol. 8.4, pp. 279-289.

Kotenko, S. V., G. Gallagher, V. V. Baurin, A. Lewis-Antes, M. Shen, N. K. Shah, J. A. Langer, F. Sheikh, H. Dickensheets, and R. P. Donnelly (2003). "IFN-Lambdas Mediate Antiviral Protection through a Distinct Class II Cytokine Receptor Complex". In: Nat. Immunol. 4.1, pp. 69-77.

Kotter, M. R., C. Zhao, N. van Rooijen, and R. J. M. Franklin (2005). "Macrophage-Depletion Induced Impairment of Experimental CNS Remyelination Is Associated with a Reduced Oligodendrocyte Progenitor Cell Response and Altered Growth Factor Expression". In: Neurobiol. Dis. 18.1, pp. 166-175.

Kraus, J., A. K. Ling, S. Hamm, K. Voigt, P. Oschmann, and B. Engelhardt (2004). "Interferon-Beta Stabilizes Barrier Characteristics of Brain Endothelial Cells in Vitro". In: Ann. Neurol. 56.2, pp. 192205.

Krebs, D. L. and D. J. Hilton (2001). "SOCS Proteins: Negative Regulators of Cytokine Signaling". In: Stem Cells 19.5, pp. 378-387.

Kuroda, H., K. Fujihara, R. Takano, Y. Takai, T. Takahashi, T. Misu, I. Nakashima, S. Sato, Y. Itoyama, and M. Aoki (2013). "Increase of Complement Fragment C5a in Cerebrospinal Fluid during Exacerbation of Neuromyelitis Optica". In: J. Neuroimmunol. 254 (1-2), pp. 178-182.

Kurt-Jones, E. A., M. Chan, S. Zhou, J. Wang, G. Reed, R. Bronson, M. M. Arnold, D. M. Knipe, and R. W. Finberg (2004). "Herpes Simplex Virus 1 Interaction with Toll-like Receptor 2 Contributes to Lethal Encephalitis". In: Proc. Natl. Acad. Sci. U.S.A. 101.5, pp. 1315-1320.

Lanie, J. A., W.-L. Ng, K. M. Kazmierczak, T. M. Andrzejewski, T. M. Davidsen, K. J. Wayne, H. Tettelin, J. I. Glass, and M. E. Winkler (2007). "Genome Sequence of Avery's Virulent Serotype 2 Strain D39 of Streptococcus Pneumoniae and Comparison with that of Unencapsulated Laboratory Strain R6". In: J. Bacteriol. 189.1, pp. 38-51.

Lawson, L. J., V. H. Perry, P. Dri, and S. Gordon (1990). "Heterogeneity in the Distribution and Morphology of Microglia in the Normal Adult Mouse Brain". In: Neuroscience 39.1, pp. 151-170.

Lee, H.-K., S. Dunzendorfer, K. Soldau, and P. S. Tobias (2006). "Double-Stranded RNA-Mediated TLR3 Activation Is Enhanced by CD14". In: Immunity 24.2, pp. 153-163.

Lee, K. S., C. A. Scanga, E. M. Bachelder, Q. Chen, and C. M. Snapper (2007). "TLR2 Synergizes with Both TLR4 and TLR9 for Induction of the MyD88-Dependent Splenic Cytokine and Chemokine Response to Streptococcus Pneumoniae". In: Cell. Immunol. 245.2, pp. 103-110.

Lehnardt, S., P. Henneke, E. Lien, D. L. Kasper, J. J. Volpe, I. Bechmann, R. Nitsch, J. R. Weber, D. T. Golenbock, and T. Vartanian (2006). "A Mechanism for Neurodegeneration Induced by Group B Streptococci through Activation of the TLR2/MyD88 Pathway in Microglia". In: J. Immunol. 177.1, pp. 583-592.

Lehnardt, S., C. Lachance, S. Patrizi, S. Lefebvre, P. L. Follett, F. E. Jensen, P. A. Rosenberg, J. J. Volpe, and T. Vartanian (2002). "The Toll-like Receptor TLR4 Is Necessary for Lipopolysaccharide-Induced Oligodendrocyte Injury in the CNS". In: J. Neurosci. 22.7, pp. 2478-2486. 
Lehnardt, S., S. Lehmann, D. Kaul, K. Tschimmel, O. Hoffmann, S. Cho, C. Krueger, R. Nitsch, A. Meisel, and J. R. Weber (2007). "Toll-like Receptor 2 Mediates CNS Injury in Focal Cerebral Ischemia". In: J. Neuroimmunol. 190 (1-2), pp. 28-33.

Lehnardt, S., L. Massillon, P. Follett, F. E. Jensen, R. Ratan, P. A. Rosenberg, J. J. Volpe, and T. Vartanian (2003). "Activation of Innate Immunity in the CNS Triggers Neurodegeneration through a Toll-like Receptor 4-Dependent Pathway". In: Proc. Natl. Acad. Sci. U.S.A. 100.14, pp. 8514-8519.

Lemaitre, B., E. Nicolas, L. Michaut, J. M. Reichhart, and J. A. Hoffmann (1996). "The Dorsoventral Regulatory Gene Cassette Spätzle/Toll/Cactus Controls the Potent Antifungal Response in Drosophila Adults". In: Cell 86.6, pp. 973-983.

Lennon, V. A., T. J. Kryzer, S. J. Pittock, A. S. Verkman, and S. R. Hinson (2005). "IgG Marker of OpticSpinal Multiple Sclerosis Binds to the Aquaporin-4 Water Channel”. In: J. Exp. Med. 202.4, pp. 473477.

Lennon, V. A., D. M. Wingerchuk, T. J. Kryzer, S. J. Pittock, C. F. Lucchinetti, K. Fujihara, I. Nakashima, and B. G. Weinshenker (2004). "A Serum Autoantibody Marker of Neuromyelitis Optica: Distinction from Multiple Sclerosis". In: Lancet 364.9451, pp. 2106-2112.

Leung, S., S. A. Qureshi, I. M. Kerr, J. E. Darnell, and G. R. Stark (1995). "Role of STAT2 in the Alpha Interferon Signaling Pathway". In: Mol. Cell. Biol. 15.3, pp. 1312-1317.

Ley, K., C. Laudanna, M. I. Cybulsky, and S. Nourshargh (2007). "Getting to the Site of Inflammation: The Leukocyte Adhesion Cascade Updated". In: Nat. Rev. Immunol. 7.9, pp. 678-689.

$\mathrm{Li}, \mathrm{S}$., A. Strelow, E. J. Fontana, and H. Wesche (2002). "IRAK-4: A Novel Member of the IRAK Family with the Properties of an IRAK-Kinase". In: Proc. Natl. Acad. Sci. U.S.A. 99.8, pp. 5567-5572.

Li, X., S. Leung, S. Qureshi, J. E. Darnell, and G. R. Stark (1996). "Formation of STAT1-STAT2 Heterodimers and Their Role in the Activation of IRF-1 Gene Transcription by Interferon-Alpha". In: J. Biol. Chem. 271.10, pp. 5790-5794.

Loo, Y.-M. and M. Gale (2011). "Immune Signaling by RIG-I-like Receptors". In: Immunity 34.5, pp. 680692.

Loughlin, A. J., M. N. Woodroofe, and M. L. Cuzner (1993). "Modulation of Interferon-Gamma-Induced Major Histocompatibility Complex Class II and Fc Receptor Expression on Isolated Microglia by Transforming Growth Factor-Beta 1, Interleukin-4, Noradrenaline and Glucocorticoids". In: Immunology 79.1, pp. 125-130.

Lucchinetti, C. F., R. N. Mandler, D. McGavern, W. Bruck, G. Gleich, R. M. Ransohoff, C. Trebst, B. Weinshenker, D. Wingerchuk, J. E. Parisi, and H. Lassmann (2002). "A Role for Humoral Mechanisms in the Pathogenesis of Devic's Neuromyelitis Optica". In: Brain 125 (Pt 7), pp. 1450-1461.

Luu, K., C. J. Greenhill, A. Majoros, T. Decker, B. J. Jenkins, and A. Mansell (2014). "STAT1 Plays a Role in TLR Signal Transduction and Inflammatory Responses”. In: Immunol. Cell Biol. 92.9, pp. 761-769.

Majer, O., C. Bourgeois, F. Zwolanek, C. Lassnig, D. Kerjaschki, M. Mack, M. Müller, and K. Kuchler (2012). "Type I Interferons Promote Fatal Immunopathology by Regulating Inflammatory Monocytes and Neutrophils during Candida Infections". In: PLoS Pathog. 8.7, e1002811.

Malakhova, O. A., K. I. Kim, J.-K. Luo, W. Zou, K. G. S. Kumar, S. Y. Fuchs, K. Shuai, and D.-E. Zhang (2006). "UBP43 Is a Novel Regulator of Interferon Signaling Independent of Its ISG15 Isopeptidase Activity". In: EMBO J. 25.11, pp. 2358-2367.

Manca, C., L. Tsenova, S. Freeman, A. K. Barczak, M. Tovey, P. J. Murray, C. Barry, and G. Kaplan (2005). "Hypervirulent M. Tuberculosis W/Beijing Strains Upregulate Type I IFNs and Increase Expression of Negative Regulators of the Jak-Stat Pathway". In: J. Interferon Cytokine Res. 25.11, pp. 694-701. 
Mancuso, G., A. Midiri, C. Biondo, C. Beninati, S. Zummo, R. Galbo, F. Tomasello, M. Gambuzza, G. Macrì, A. Ruggeri, T. Leanderson, and G. Teti (2007). "Type I IFN Signaling Is Crucial for Host Resistance against Different Species of Pathogenic Bacteria". In: J. Immunol. 178.5, pp. 3126-3133.

Martinez-Moczygemba, M., M. J. Gutch, D. L. French, and N. C. Reich (1997). "Distinct STAT Structure Promotes Interaction of STAT2 with the p48 Subunit of the Interferon-Alpha-Stimulated Transcription Factor ISGF3". In: J. Biol. Chem. 272.32, pp. 20070-20076.

Matzinger, P. (1994). "Tolerance, Danger, and the Extended Family". In: Annu. Rev. Immunol. 12, pp. 991-1045.

Matzinger, P. (2002). "The Danger Model: A Renewed Sense of Self". In: Science 296.5566, pp. 301305.

McManus, C. M., J. S. Liu, M. T. Hahn, L. L. Hua, C. F. Brosnan, J. W. Berman, and S. C. Lee (2000). "Differential Induction of Chemokines in Human Microglia by Type I and II Interferons". In: Glia 29.3, pp. 273-280.

Medzhitov, R., P. Preston-Hurlburt, and C. A. Janeway (1997). "A Human Homologue of the Drosophila Toll Protein Signals Activation of Adaptive Immunity”. In: Nature 388.6640, pp. 394-397.

Meinke, A., F. Barahmand-Pour, S. Wöhrl, D. Stoiber, and T. Decker (1996). "Activation of Different Stat5 Isoforms Contributes to Cell-Type-Restricted Signaling in Response to Interferons". In: Mol. Cell. Biol. 16.12, pp. 6937-6944.

Menzfeld, C., M. John, D. van Rossum, T. Regen, J. Scheffel, H. Janova, A. Götz, S. Ribes, R. Nau, A. Borisch, P. Boutin, K. Neumann, V. Bremes, J. Wienands, H. M. Reichardt, F. Lühder, D. Tischner, V. Waetzig, T. Herdegen, P. Teismann, I. Greig, M. Müller, T. Pukrop, A. Mildner, H. Kettenmann, W. Brück, M. Prinz, S. Rotshenker, M. S. Weber, and U.-K. Hanisch (2015). "Tyrphostin AG126 Exerts Neuroprotection in CNS Inflammation by a Dual Mechanism". In: Glia 63.6, pp. 1083-1099.

Mercier, F. E., C. Ragu, and D. T. Scadden (2012). "The Bone Marrow at the Crossroads of Blood and Immunity". In: Nat. Rev. Immunol. 12.1, pp. 49-60.

Minghetti, L. and G. Levi (1998). "Microglia as Effector Cells in Brain Damage and Repair: Focus on Prostanoids and Nitric Oxide". In: Prog. Neurobiol. 54.1, pp. 99-125.

Mishra, B. B., U. M. Gundra, and J. M. Teale (2008). "Expression and Distribution of Toll-like Receptors 11-13 in the Brain during Murine Neurocysticercosis". In: J Neuroinflammation 5, p. 53.

Misu, T., K. Fujihara, A. Kakita, H. Konno, M. Nakamura, S. Watanabe, T. Takahashi, I. Nakashima, H. Takahashi, and Y. Itoyama (2007). "Loss of Aquaporin 4 in Lesions of Neuromyelitis Optica: Distinction from Multiple Sclerosis”. In: Brain 130 (Pt 5), pp. 1224-1234.

Mitchell, A. J., B. Yau, J. A. McQuillan, H. J. Ball, L. K. Too, A. Abtin, P. Hertzog, S. L. Leib, C. A. Jones, S. K. Gerega, W. Weninger, and N. H. Hunt (2012). "Inflammasome-Dependent IFN-Gamma Drives Pathogenesis in Streptococcus Pneumoniae Meningitis". In: J. Immunol. 189.10, pp. 4970-4980.

Mogensen, T. H., S. R. Paludan, M. Kilian, and L. Ostergaard (2006). "Live Streptococcus Pneumoniae, Haemophilus Influenzae, and Neisseria Meningitidis Activate the Inflammatory Response through Toll-like Receptors 2, 4, and 9 in Species-Specific Patterns". In: J. Leukoc. Biol. 80.2, pp. 267-277.

Müller, U., U. Steinhoff, L. F. Reis, S. Hemmi, J. Pavlovic, R. M. Zinkernagel, and M. Aguet (1994). "Functional Role of Type I and Type II Interferons in Antiviral Defense". In: Science 264.5167, pp. 19181921.

Nagelhus, E. A., T. M. Mathiisen, and O. P. Ottersen (2004). "Aquaporin-4 in the Central Nervous System: Cellular and Subcellular Distribution and Coexpression with KIR4.1". In: Neuroscience 129.4, pp. 905-913.

Nakamichi, K., M. Saiki, M. Sawada, Y. Yamamuro, K. Morimoto, and I. Kurane (2005). "Double-Stranded RNA Stimulates Chemokine Expression in Microglia through Vacuolar pH-Dependent Activation of Intracellular Signaling Pathways". In: J. Neurochem. 95.1, pp. 273-283. 
Nesargikar, P. N., B. Spiller, and R. Chavez (2012). "The Complement System: History, Pathways, Cascade and Inhibitors". In: Eur J Microbiol Immunol (Bp) 2.2, pp. 103-111.

Netea, M. G., N. A. R. Gow, C. A. Munro, S. Bates, C. Collins, G. Ferwerda, R. P. Hobson, G. Bertram, H. B. Hughes, T. Jansen, L. Jacobs, E. T. Buurman, K. Gijzen, D. L. Williams, R. Torensma, A. Mc Kinnon, D. M. MacCallum, F. C. Odds, J. W. M. Van der Meer, A. J. P. Brown, and B. J. Kullberg (2006). "Immune Sensing of Candida Albicans Requires Cooperative Recognition of Mannans and Glucans by Lectin and Toll-like Receptors". In: J. Clin. Invest. 116.6, pp. 1642-1650.

Neumann, J., M. Gunzer, H. O. Gutzeit, O. Ullrich, K. G. Reymann, and K. Dinkel (2006). "Microglia Provide Neuroprotection after Ischemia". In: FASEB J. 20.6, pp. 714-716.

Nimmerjahn, A., F. Kirchhoff, and F. Helmchen (2005). "Resting Microglial Cells Are Highly Dynamic Surveillants of Brain Parenchyma in Vivo". In: Science 308.5726, pp. 1314-1318.

Noppert, S. J., K. A. Fitzgerald, and P. J. Hertzog (2007). "The Role of Type I Interferons in TLR Responses". In: Immunol. Cell Biol. 85.6, pp. 446-457.

O’Brien, K. L., L. J. Wolfson, J. P. Watt, E. Henkle, M. Deloria-Knoll, N. McCall, E. Lee, K. Mulholland,

O. S. Levine, T. Cherian, and Hib and Pneumococcal Global Burden of Disease Study Team (2009). "Burden of Disease Caused by Streptococcus Pneumoniae in Children Younger than 5 Years: Global Estimates". In: Lancet 374.9693, pp. 893-902.

Ohmori, Y. and T. A. Hamilton (2001). "Requirement for STAT1 in LPS-Induced Gene Expression in Macrophages". In: J. Leukoc. Biol. 69.4, pp. 598-604.

Okamura, Y., M. Watari, E. S. Jerud, D. W. Young, S. T. Ishizaka, J. Rose, J. C. Chow, and J. F. Strauss (2001). "The Extra Domain A of Fibronectin Activates Toll-like Receptor 4". In: J. Biol. Chem. 276.13, pp. 10229-10233.

Oldenburg, M., A. Krüger, R. Ferstl, A. Kaufmann, G. Nees, A. Sigmund, B. Bathke, H. Lauterbach, M. Suter, S. Dreher, U. Koedel, S. Akira, T. Kawai, J. Buer, H. Wagner, S. Bauer, H. Hochrein, and C. J. Kirschning (2012). "TLR13 Recognizes Bacterial 23S rRNA Devoid of Erythromycin ResistanceForming Modification”. In: Science 337.6098, pp. 1111-1115.

Olson, J. K. and S. D. Miller (2004). "Microglia Initiate Central Nervous System Innate and Adaptive Immune Responses through Multiple TLRs". In: J. Immunol. 173.6, pp. 3916-3924.

Omari, K. M., G. John, R. Lango, and C. S. Raine (2006). "Role for CXCR2 and CXCL1 on Glia in Multiple Sclerosis". In: Glia 53.1, pp. 24-31.

O'Neill, L. A. J. and A. G. Bowie (2007). "The Family of Five: TIR-Domain-Containing Adaptors in Tolllike Receptor Signalling”. In: Nat. Rev. Immunol. 7.5, pp. 353-364.

O'Neill, L. A. J., D. Golenbock, and A. G. Bowie (2013). "The History of Toll-like Receptors - Redefining Innate Immunity”. In: Nat. Rev. Immunol. 13.6, pp. 453-460.

Opitz, B., A. Püschel, B. Schmeck, A. C. Hocke, S. Rosseau, S. Hammerschmidt, R. R. Schumann, N. Suttorp, and S. Hippenstiel (2004). "Nucleotide-Binding Oligomerization Domain Proteins Are Innate Immune Receptors for Internalized Streptococcus Pneumoniae". In: J. Biol. Chem. 279.35, pp. 36426-36432.

Ortaldo, J. R., A. Mantovani, D. Hobbs, M. Rubinstein, S. Pestka, and R. B. Herberman (1983). "Effects of Several Species of Human Leukocyte Interferon on Cytotoxic Activity of NK Cells and Monocytes". In: Int. J. Cancer 31.3, pp. 285-289.

Owens, T., R. Khorooshi, A. Wlodarczyk, and N. Asgari (2014). "Interferons in the Central Nervous System: A Few Instruments Play Many Tunes". In: Glia 62.3, pp. 339-355.

Ozinsky, A., D. M. Underhill, J. D. Fontenot, A. M. Hajjar, K. D. Smith, C. B. Wilson, L. Schroeder, and A. Aderem (2000). "The Repertoire for Pattern Recognition of Pathogens by the Innate Immune System Is Defined by Cooperation between Toll-like Receptors". In: Proc. Natl. Acad. Sci. U.S.A. 97.25, pp. 13766-13771. 
Palace, J., M. I. Leite, A. Nairne, and A. Vincent (2010). "Interferon Beta Treatment in Neuromyelitis Optica: Increase in Relapses and Aquaporin 4 Antibody Titers". In: Arch. Neurol. 67.8, pp. 10161017.

Pandey, A. K., Y. Yang, Z. Jiang, S. M. Fortune, F. Coulombe, M. A. Behr, K. A. Fitzgerald, C. M. Sassetti, and M. A. Kelliher (2009). "NOD2, RIP2 and IRF5 Play a Critical Role in the Type I Interferon Response to Mycobacterium Tuberculosis". In: PLoS Pathog. 5.7, e1000500.

Pankov, R. and K. M. Yamada (2002). "Fibronectin at a Glance". In: J. Cell. Sci. 115 (Pt 20), pp. 38613863.

Paolicelli, R. C., G. Bolasco, F. Pagani, L. Maggi, M. Scianni, P. Panzanelli, M. Giustetto, T. A. Ferreira, E. Guiducci, L. Dumas, D. Ragozzino, and C. T. Gross (2011). "Synaptic Pruning by Microglia Is Necessary for Normal Brain Development”. In: Science 333.6048, pp. 1456-1458.

Papadopoulos, M. C. and A. S. Verkman (2012). "Aquaporin 4 and Neuromyelitis Optica". In: Lancet Neurol 11.6, pp. 535-544.

Park, B. S. and J.-O. Lee (2013). "Recognition of Lipopolysaccharide Pattern by TLR4 Complexes". In: Exp. Mol. Med. 45, e66.

Parkhurst, C. N., G. Yang, I. Ninan, J. N. Savas, J. R. Yates, J. J. Lafaille, B. L. Hempstead, D. R. Littman, and W.-B. Gan (2013). "Microglia Promote Learning-Dependent Synapse Formation through Brain-Derived Neurotrophic Factor". In: Cell 155.7, pp. 1596-1609.

Pashenkov, M., Y.-M. Huang, V. Kostulas, M. Haglund, M. Söderström, and H. Link (2001). "Two Subsets of Dendritic Cells Are Present in Human Cerebrospinal Fluid". In: Brain 124.3, pp. 480-492.

Pestka, S. (2007). "The Interferons: 50 Years after Their Discovery, There Is Much More to Learn". In: J. Biol. Chem. 282.28, pp. 20047-20051.

Platanias, L. C. (2005). "Mechanisms of Type-I- and Type-II-Interferon-Mediated Signalling". In: Nat. Rev. Immunol. 5.5, pp. 375-386.

Plumlee, C. R., C. Lee, A. A. Beg, T. Decker, H. A. Shuman, and C. Schindler (2009). "Interferons Direct an Effective Innate Response to Legionella Pneumophila Infection”. In: J. Biol. Chem. 284.44, pp. 30058-30066.

Poltorak, A., X. He, I. Smirnova, M. Y. Liu, C. Van Huffel, X. Du, D. Birdwell, E. Alejos, M. Silva, C. Galanos, M. Freudenberg, P. Ricciardi-Castagnoli, B. Layton, and B. Beutler (1998). "Defective LPS Signaling in C3H/HeJ and C57BL/10ScCr Mice: Mutations in TIr4 Gene". In: Science 282.5396, pp. 2085-2088.

Préhaud, C., F. Mégret, M. Lafage, and M. Lafon (2005). "Virus Infection Switches TLR-3-Positive Human Neurons to Become Strong Producers of Beta Interferon". In: J. Virol. 79.20, pp. 12893-12904.

Radtke, S., S. Haan, A. Jörissen, H. M. Hermanns, S. Diefenbach, T. Smyczek, H. Schmitz-Vandeleur, P. C. Heinrich, I. Behrmann, and C. Haan (2005). "The Jak1 SH2 Domain Does Not Fulfill a Classical SH2 Function in Jak/STAT Signaling but Plays a Structural Role for Receptor Interaction and upRegulation of Receptor Surface Expression". In: J. Biol. Chem. 280.27, pp. 25760-25768.

Raetz, C. R. H. and C. Whitfield (2002). "Lipopolysaccharide Endotoxins". In: Annu. Rev. Biochem. 71, pp. 635-700.

Raetz, M., A. Kibardin, C. R. Sturge, R. Pifer, H. Li, E. Burstein, K. Ozato, S. Larin, and F. Yarovinsky (2013). "Cooperation of TLR12 and TLR11 in the IRF8-Dependent IL-12 Response to Toxoplasma Gondii Profilin". In: J. Immunol. 191.9, pp. 4818-4827.

Randolph, G. J., C. Jakubzick, and C. Qu (2008). "Antigen Presentation by Monocytes and MonocyteDerived Cells". In: Curr. Opin. Immunol. 20.1, pp. 52-60.

Ransohoff, R. M. and A. E. Cardona (2010). "The Myeloid Cells of the Central Nervous System Parenchyma". In: Nature 468.7321, pp. 253-262. 
Rathinam, V. A. K., S. K. Vanaja, L. Waggoner, A. Sokolovska, C. Becker, L. M. Stuart, J. M. Leong, and K. A. Fitzgerald (2012). "TRIF Licenses Caspase-11-Dependent NLRP3 Inflammasome Activation by Gram-Negative Bacteria”. In: Cell 150.3, pp. 606-619.

Rauch, I., M. Müller, and T. Decker (2013). "The Regulation of Inflammation by Interferons and Their STATs". In: JAKSTAT 2.1, e23820.

Regen, T., D. van Rossum, J. Scheffel, M.-E. Kastriti, N. H. Revelo, M. Prinz, W. Brück, and U.-K. Hanisch (2011). "CD14 and TRIF Govern Distinct Responsiveness and Responses in Mouse Microglial TLR4 Challenges by Structural Variants of LPS". In: Brain Behav. Immun. 25.5, pp. 957-970.

Rhee, S. H., B. W. Jones, V. Toshchakov, S. N. Vogel, and M. J. Fenton (2003). "Toll-like Receptors 2 and 4 Activate STAT1 Serine Phosphorylation by Distinct Mechanisms in Macrophages”. In: J. Biol. Chem. 278.25, pp. 22506-22512.

Rho, M. B., S. Wesselingh, J. D. Glass, J. C. McArthur, S. Choi, J. Griffin, and W. R. Tyor (1995). "A Potential Role for Interferon-Alpha in the Pathogenesis of HIV-Associated Dementia". In: Brain Behav. Immun. 9.4, pp. 366-377.

Ribes, S., S. Ebert, D. Czesnik, T. Regen, A. Zeug, S. Bukowski, A. Mildner, H. Eiffert, U.-K. Hanisch, S. Hammerschmidt, and R. Nau (2009). "Toll-like Receptor Prestimulation Increases Phagocytosis of Escherichia Coli DH5alpha and Escherichia Coli K1 Strains by Murine Microglial Cells". In: Infect. Immun. 77.1, pp. 557-564.

Ribes, S., S. Ebert, T. Regen, A. Agarwal, S. C. Tauber, D. Czesnik, A. Spreer, S. Bunkowski, H. Eiffert, U.-K. Hanisch, S. Hammerschmidt, and R. Nau (2010). "Toll-like Receptor Stimulation Enhances Phagocytosis and Intracellular Killing of Nonencapsulated and Encapsulated Streptococcus Pneumoniae by Murine Microglia". In: Infect. Immun. 78.2, pp. 865-871.

Ribes, S., T. Regen, T. Meister, S. C. Tauber, S. Schütze, A. Mildner, M. Mack, U.-K. Hanisch, and R. Nau (2013). "Resistance of the Brain to Escherichia Coli K1 Infection Depends on MyD88 Signaling and the Contribution of Neutrophils and Monocytes". In: Infect. Immun. 81.5, pp. 1810-1819.

Richter, M. F., G. Duménil, G. Uzé, M. Fellous, and S. Pellegrini (1998). "Specific Contribution of Tyk2 $\mathrm{JH}$ Regions to the Binding and the Expression of the Interferon Alpha/Beta Receptor Component IFNAR1". In: J. Biol. Chem. 273.38, pp. 24723-24729.

Robbins, J. B., G. H. McCracken, E. C. Gotschlich, F. Orskov, I. Orskov, and L. A. Hanson (1974). "Escherichia Coli K1 Capsular Polysaccharide Associated with Neonatal Meningitis". In: N. Engl. J. Med. 290.22, pp. 1216-1220.

Rodig, S. J., M. A. Meraz, J. M. White, P. A. Lampe, J. K. Riley, C. D. Arthur, K. L. King, K. C. Sheehan, L. Yin, D. Pennica, E. M. Johnson, and R. D. Schreiber (1998). "Disruption of the Jak1 Gene Demonstrates Obligatory and Nonredundant Roles of the Jaks in Cytokine-Induced Biologic Responses". In: Cell 93.3, pp. 373-383.

Roemer, S. F., J. E. Parisi, V. A. Lennon, E. E. Benarroch, H. Lassmann, W. Bruck, R. N. Mandler, B. G. Weinshenker, S. J. Pittock, D. M. Wingerchuk, and C. F. Lucchinetti (2007). "Pattern-Specific Loss of Aquaporin-4 Immunoreactivity Distinguishes Neuromyelitis Optica from Multiple Sclerosis". In: Brain 130 (Pt 5), pp. 1194-1205.

Saadoun, S., P. Waters, B. A. Bell, A. Vincent, A. S. Verkman, and M. C. Papadopoulos (2010). "IntraCerebral Injection of Neuromyelitis Optica Immunoglobulin $G$ and Human Complement Produces Neuromyelitis Optica Lesions in Mice". In: Brain 133 (Pt 2), pp. 349-361.

Sadowski, H. B., K. Shuai, J. E. Darnell, and M. Z. Gilman (1993). "A Common Nuclear Signal Transduction Pathway Activated by Growth Factor and Cytokine Receptors”. In: Science 261.5129, pp. 17391744.

Sáez-Llorens, X. and G. H. McCracken (2003). "Bacterial Meningitis in Children”. In: Lancet 361.9375, pp. 2139-2148. 
Saharinen, P. and O. Silvennoinen (2002). "The Pseudokinase Domain Is Required for Suppression of Basal Activity of Jak2 and Jak3 Tyrosine Kinases and for Cytokine-Inducible Activation of Signal Transduction". In: J. Biol. Chem. 277.49, pp. 47954-47963.

Samuel, C. E. (2001). "Antiviral Actions of Interferons". In: Clin. Microbiol. Rev. 14.4, pp. 778-809.

Santer, D. M., T. Yoshio, S. Minota, T. Möller, and K. B. Elkon (2009). "Potent Induction of IFN-Alpha and Chemokines by Autoantibodies in the Cerebrospinal Fluid of Patients with Neuropsychiatric Lupus". In: J. Immunol. 182.2, pp. 1192-1201.

Sarasin-Filipowicz, M., X. Wang, M. Yan, F. H. T. Duong, V. Poli, D. J. Hilton, D.-E. Zhang, and M. H. Heim (2009). "Alpha Interferon Induces Long-Lasting Refractoriness of JAK-STAT Signaling in the Mouse Liver through Induction of USP18/UBP43". In: Mol. Cell. Biol. 29.17, pp. 4841-4851.

Sato, M., N. Hata, M. Asagiri, T. Nakaya, T. Taniguchi, and N. Tanaka (1998). "Positive Feedback Regulation of Type I IFN Genes by the IFN-Inducible Transcription Factor IRF-7". In: FEBS Lett. 441.1, pp. 106-110.

Savill, J. S., A. H. Wyllie, J. E. Henson, M. J. Walport, P. M. Henson, and C. Haslett (1989). "Macrophage Phagocytosis of Aging Neutrophils in Inflammation. Programmed Cell Death in the Neutrophil Leads to Its Recognition by Macrophages". In: J. Clin. Invest. 83.3, pp. 865-875.

Schindler, C., X. Y. Fu, T. Improta, R. Aebersold, and J. E. Darnell (1992). "Proteins of Transcription Factor ISGF-3: One Gene Encodes the 91-and 84-kDa ISGF-3 Proteins That Are Activated by Interferon Alpha”. In: Proc. Natl. Acad. Sci. U.S.A. 89.16, pp. 7836-7839.

Schulz, C., E. Gomez Perdiguero, L. Chorro, H. Szabo-Rogers, N. Cagnard, K. Kierdorf, M. Prinz, B. Wu, S. E. W. Jacobsen, J. W. Pollard, J. Frampton, K. J. Liu, and F. Geissmann (2012). "A Lineage of Myeloid Cells Independent of Myb and Hematopoietic Stem Cells”. In: Science 336.6077, pp. 86-90.

Semerad, C. L., F. Liu, A. D. Gregory, K. Stumpf, and D. C. Link (2002). "G-CSF Is an Essential Regulator of Neutrophil Trafficking from the Bone Marrow to the Blood". In: Immunity 17.4, pp. 413-423.

Semple, B. D., N. Bye, J. M. Ziebell, and M. C. Morganti-Kossmann (2010). "Deficiency of the Chemokine Receptor CXCR2 Attenuates Neutrophil Infiltration and Cortical Damage Following Closed Head Injury". In: Neurobiol. Dis. 40.2, pp. 394-403.

Sen, G. C. (2001). "Viruses and Interferons". In: Annu. Rev. Microbiol. 55, pp. 255-281.

Serhan, C. N., J. F. Maddox, N. A. Petasis, I. Akritopoulou-Zanze, A. Papayianni, H. R. Brady, S. P. Colgan, and J. L. Madara (1995). "Design of Lipoxin A4 Stable Analogs That Block Transmigration and Adhesion of Human Neutrophils". In: Biochemistry 34.44, pp. 14609-14615.

Serhan, C. N. (2010). "Novel Lipid Mediators and Resolution Mechanisms in Acute Inflammation: To Resolve or Not?" In: Am. J. Pathol. 177.4, pp. 1576-1591.

Shahangian, A., E. K. Chow, X. Tian, J. R. Kang, A. Ghaffari, S. Y. Liu, J. A. Belperio, G. Cheng, and J. C. Deng (2009). "Type I IFNs Mediate Development of Postinfluenza Bacterial Pneumonia in Mice". In: J. Clin. Invest. 119.7, pp. 1910-1920.

Shaw, M. H., V. Boyartchuk, S. Wong, M. Karaghiosoff, J. Ragimbeau, S. Pellegrini, M. Muller, W. F. Dietrich, and G. S. Yap (2003). "A Natural Mutation in the Tyk2 Pseudokinase Domain Underlies Altered Susceptibility of B10.Q/J Mice to Infection and Autoimmunity". In: Proc. Natl. Acad. Sci. U.S.A. 100.20, pp. 11594-11599.

Sheppard, P., W. Kindsvogel, W. Xu, K. Henderson, S. Schlutsmeyer, T. E. Whitmore, R. Kuestner, U. Garrigues, C. Birks, J. Roraback, C. Ostrander, D. Dong, J. Shin, S. Presnell, B. Fox, B. Haldeman, E. Cooper, D. Taft, T. Gilbert, F. J. Grant, M. Tackett, W. Krivan, G. McKnight, C. Clegg, D. Foster, and K. M. Klucher (2003). "IL-28, IL-29 and Their Class II Cytokine Receptor IL-28R". In: Nat. Immunol. 4.1 , pp. 63-68. 
Shi, Z., Z. Cai, A. Sanchez, T. Zhang, S. Wen, J. Wang, J. Yang, S. Fu, and D. Zhang (2011). "A Novel Toll-like Receptor That Recognizes Vesicular Stomatitis Virus”. In: J. Biol. Chem. 286.6, pp. 45174524.

Shimazu, R., S. Akashi, H. Ogata, Y. Nagai, K. Fukudome, K. Miyake, and M. Kimoto (1999). "MD-2, a Molecule That Confers Lipopolysaccharide Responsiveness on Toll-like Receptor 4". In: J. Exp. Med. 189.11, pp. 1777-1782.

Shimoda, K., K. Kato, K. Aoki, T. Matsuda, A. Miyamoto, M. Shibamori, M. Yamashita, A. Numata, K. Takase, S. Kobayashi, S. Shibata, Y. Asano, H. Gondo, K. Sekiguchi, K. Nakayama, T. Nakayama, T. Okamura, S. Okamura, Y. Niho, and K. Nakayama (2000). "Tyk2 Plays a Restricted Role in IFN Alpha Signaling, Although It Is Required for IL-12-Mediated T Cell Function”. In: Immunity 13.4, pp. 561571.

Shuai, K., C. Schindler, V. R. Prezioso, and J. E. Darnell (1992). "Activation of Transcription by IFNGamma: Tyrosine Phosphorylation of a 91-kD DNA Binding Protein”. In: Science 258.5089, pp. 18081812.

Shuai, K., G. R. Stark, I. M. Kerr, and J. E. Darnell (1993). "A Single Phosphotyrosine Residue of Stat91

Required for Gene Activation by Interferon-Gamma". In: Science 261.5129, pp. 1744-1746.

Shuai, K. and B. Liu (2003). "Regulation of JAK-STAT Signalling in the Immune System". In: Nat. Rev. Immunol. 3.11, pp. 900-911.

Siegal, F. P., N. Kadowaki, M. Shodell, P. A. Fitzgerald-Bocarsly, K. Shah, S. Ho, S. Antonenko, and Y. J. Liu (1999). "The Nature of the Principal Type 1 Interferon-Producing Cells in Human Blood". In: Science 284.5421, pp. 1835-1837.

Sierra, A., O. Abiega, A. Shahraz, and H. Neumann (2013). "Janus-Faced Microglia: Beneficial and Detrimental Consequences of Microglial Phagocytosis". In: Front Cell Neurosci 7, p. 6.

Sierra, A., J. M. Encinas, J. J. P. Deudero, J. H. Chancey, G. Enikolopov, L. S. Overstreet-Wadiche, S. E. Tsirka, and M. Maletic-Savatic (2010). "Microglia Shape Adult Hippocampal Neurogenesis through Apoptosis-Coupled Phagocytosis". In: Cell Stem Cell 7.4, pp. 483-495.

Simard, A. R. and S. Rivest (2007). "Neuroprotective Effects of Resident Microglia Following Acute Brain Injury". In: J. Comp. Neurol. 504.6, pp. 716-729.

Smiley, S. T., J. A. King, and W. W. Hancock (2001). "Fibrinogen Stimulates Macrophage Chemokine Secretion through Toll-like Receptor 4". In: J. Immunol. 167.5, pp. 2887-2894.

Soehnlein, O., L. Lindbom, and C. Weber (2009). "Mechanisms Underlying Neutrophil-Mediated Monocyte Recruitment". In: Blood 114.21, pp. 4613-4623.

Stack, J., S. L. Doyle, D. J. Connolly, L. S. Reinert, K. M. O'Keeffe, R. M. McLoughlin, S. R. Paludan, and A. G. Bowie (2014). "TRAM Is Required for TLR2 Endosomal Signaling to Type I IFN Induction". In: J. Immunol. 193.12, pp. 6090-6102.

Stark, G. R., I. M. Kerr, B. R. Williams, R. H. Silverman, and R. D. Schreiber (1998). "How Cells Respond to Interferons". In: Annu. Rev. Biochem. 67, pp. 227-264.

Stenzel, W., S. Soltek, M. Sanchez-Ruiz, S. Akira, H. Miletic, D. Schlüter, and M. Deckert (2008). "Both TLR2 and TLR4 Are Required for the Effective Immune Response in Staphylococcus Aureus-Induced Experimental Murine Brain Abscess". In: Am. J. Pathol. 172.1, pp. 132-145.

Stewart, C. R., L. M. Stuart, K. Wilkinson, J. M. van Gils, J. Deng, A. Halle, K. J. Rayner, L. Boyer, R. Zhong, W. A. Frazier, A. Lacy-Hulbert, J. El Khoury, D. T. Golenbock, and K. J. Moore (2010). "CD36 Ligands Promote Sterile Inflammation through Assembly of a Toll-like Receptor 4 and 6 Heterodimer". In: Nat. Immunol. 11.2, pp. 155-161.

Summers, C., S. M. Rankin, A. M. Condliffe, N. Singh, A. M. Peters, and E. R. Chilvers (2010). "Neutrophil Kinetics in Health and Disease". In: Trends Immunol. 31.8, pp. 318-324. 
Tahara, K., H.-D. Kim, J.-J. Jin, J. A. Maxwell, L. Li, and K.-i. Fukuchi (2006). "Role of Toll-like Receptor Signalling in Abeta Uptake and Clearance". In: Brain 129 (Pt 11), pp. 3006-3019.

Takano, T., C. B. Clish, K. Gronert, N. Petasis, and C. N. Serhan (1998). "Neutrophil-Mediated Changes in Vascular Permeability Are Inhibited by Topical Application of Aspirin-Triggered 15-Epi-Lipoxin A4 and Novel Lipoxin B4 Stable Analogues". In: J. Clin. Invest. 101.4, pp. 819-826.

Takaoka, A., Z. Wang, M. K. Choi, H. Yanai, H. Negishi, T. Ban, Y. Lu, M. Miyagishi, T. Kodama, K. Honda, Y. Ohba, and T. Taniguchi (2007). "DAI (DLM-1/ZBP1) Is a Cytosolic DNA Sensor and an Activator of Innate Immune Response". In: Nature 448.7152, pp. 501-505.

Takeuchi, O., K. Hoshino, and S. Akira (2000). "Cutting Edge: TLR2-Deficient and MyD88-Deficient Mice Are Highly Susceptible to Staphylococcus Aureus Infection”. In: J. Immunol. 165.10, pp. 5392-5396.

Takeuchi, O., T. Kawai, P. F. Mühlradt, M. Morr, J. D. Radolf, A. Zychlinsky, K. Takeda, and S. Akira (2001). "Discrimination of Bacterial Lipoproteins by Toll-like Receptor 6". In: Int. Immunol. 13.7, pp. 933940.

Takeuchi, O. and S. Akira (2010). "Pattern Recognition Receptors and Inflammation". In: Cell 140.6, pp. 805-820.

Tanga, F. Y., N. Nutile-McMenemy, and J. A. DeLeo (2005). "The CNS Role of Toll-like Receptor 4 in Innate Neuroimmunity and Painful Neuropathy". In: Proc. Natl. Acad. Sci. U.S.A. 102.16, pp. 58565861.

Tanner, J. W., W. Chen, R. L. Young, G. D. Longmore, and A. S. Shaw (1995). "The Conserved Box 1 Motif of Cytokine Receptors Is Required for Association with JAK Kinases”. In: J. Biol. Chem. 270.12, pp. 6523-6530.

Teitelbaum, S. L. and F. P. Ross (2003). "Genetic Regulation of Osteoclast Development and Function". In: Nat. Rev. Genet. 4.8, pp. 638-649.

The IFNB Multiple Sclerosis Study Group (1993). "Interferon Beta-1b Is Effective in Relapsing-Remitting Multiple Sclerosis. I. Clinical Results of a Multicenter, Randomized, Double-Blind, Placebo-Controlled Trial." In: Neurology 43.4, pp. 655-661.

Theofilopoulos, A. N., R. Baccala, B. Beutler, and D. H. Kono (2005). "Type I Interferons (Alpha/Beta) in Immunity and Autoimmunity”. In: Annu. Rev. Immunol. 23, pp. 307-336.

Thompson, J. E., R. M. Cubbon, R. T. Cummings, L. S. Wicker, R. Frankshun, B. R. Cunningham, P. M. Cameron, P. T. Meinke, N. Liverton, Y. Weng, and J. A. DeMartino (2002). "Photochemical Preparation of a Pyridone Containing Tetracycle: A Jak Protein Kinase Inhibitor". In: Bioorg. Med. Chem. Lett. 12.8, pp. 1219-1223.

Torpey, N., S. E. Maher, A. L. M. Bothwell, and J. S. Pober (2004). "Interferon Alpha but Not Interleukin 12 Activates STAT4 Signaling in Human Vascular Endothelial Cells". In: J. Biol. Chem. 279.25, pp. 26789-26796.

Toshchakov, V., B. W. Jones, P.-Y. Perera, K. Thomas, M. J. Cody, S. Zhang, B. R. G. Williams, J. Major, T. A. Hamilton, M. J. Fenton, and S. N. Vogel (2002). "TLR4, but Not TLR2, Mediates IFNBeta-Induced STAT1alpha/Beta-Dependent Gene Expression in Macrophages". In: Nat. Immunol. 3.4, pp. 392-398.

Tremblay, M.-Ė., R. L. Lowery, and A. K. Majewska (2010). "Microglial Interactions with Synapses Are Modulated by Visual Experience". In: PLoS Biol. 8.11, e1000527.

Trinchieri, G. (2010). “Type I Interferon: Friend or Foe?” In: J Exp Med 207.10, pp. 2053-2063.

Tsou, C.-L., W. Peters, Y. Si, S. Slaymaker, A. M. Aslanian, S. P. Weisberg, M. Mack, and I. F. Charo (2007). "Critical Roles for CCR2 and MCP-3 in Monocyte Mobilization from Bone Marrow and Recruitment to Inflammatory Sites". In: J. Clin. Invest. 117.4, pp. 902-909. 
Uddin, S., A. Sassano, D. K. Deb, A. Verma, B. Majchrzak, A. Rahman, A. B. Malik, E. N. Fish, and L. C. Platanias (2002). "Protein Kinase C-Delta (PKC-Delta) Is Activated by Type I Interferons and Mediates Phosphorylation of Stat1 on Serine 727". In: J. Biol. Chem. 277.17, pp. 14408-14416.

Unterholzner, L., S. E. Keating, M. Baran, K. A. Horan, S. B. Jensen, S. Sharma, C. M. Sirois, T. Jin, E. Latz, T. S. Xiao, K. A. Fitzgerald, S. R. Paludan, and A. G. Bowie (2010). "IFI16 Is an Innate Immune Sensor for Intracellular DNA". In: Nat. Immunol. 11.11, pp. 997-1004.

Uzé, G., G. Lutfalla, and I. Gresser (1990). "Genetic Transfer of a Functional Human Interferon Alpha Receptor into Mouse Cells: Cloning and Expression of Its cDNA". In: Cell 60.2, pp. 225-234.

Valente, A. J., J. F. Xie, M. A. Abramova, U. O. Wenzel, H. E. Abboud, and D. T. Graves (1998). "A Complex Element Regulates IFN-Gamma-Stimulated Monocyte Chemoattractant Protein-1 Gene Transcription". In: J. Immunol. 161.7, pp. 3719-3728.

Van Rompaey, L., R. Galien, E. M. van der Aar, P. Clement-Lacroix, L. Nelles, B. Smets, L. Lepescheux, T. Christophe, K. Conrath, N. Vandeghinste, B. Vayssiere, S. De Vos, S. Fletcher, R. Brys, G. van 't Klooster, J. H. M. Feyen, and C. Menet (2013). "Preclinical Characterization of GLPG0634, a Selective Inhibitor of JAK1, for the Treatment of Inflammatory Diseases". In: J. Immunol. 191.7, pp. 3568-3577.

Van Furth, R. and Z. A. Cohn (1968). "The Origin and Kinetics of Mononuclear Phagocytes". In: J. Exp. Med. 128.3, pp. 415-435.

Van Pesch, V., H. Lanaya, J.-C. Renauld, and T. Michiels (2004). "Characterization of the Murine Alpha Interferon Gene Family". In: J. Virol. 78.15, pp. 8219-8228.

Velazquez, L., K. E. Mogensen, G. Barbieri, M. Fellous, G. Uzé, and S. Pellegrini (1995). "Distinct Domains of the Protein Tyrosine Kinase tyk2 Required for Binding of Interferon-Alpha/Beta and for Signal Transduction". In: J. Biol. Chem. 270.7, pp. 3327-3334.

Wake, H., A. J. Moorhouse, S. Jinno, S. Kohsaka, and J. Nabekura (2009). "Resting Microglia Directly Monitor the Functional State of Synapses in Vivo and Determine the Fate of Ischemic Terminals". In: J. Neurosci. 29.13, pp. 3974-3980.

Walton, N. M., B. M. Sutter, E. D. Laywell, L. H. Levkoff, S. M. Kearns, G. P. Marshall, B. Scheffler, and D. A. Steindler (2006). "Microglia Instruct Subventricular Zone Neurogenesis". In: Glia 54.8, pp. 815825.

Wang, C., L. Deng, M. Hong, G. R. Akkaraju, J. Inoue, and Z. J. Chen (2001). "TAK1 Is a UbiquitinDependent Kinase of MKK and IKK". In: Nature 412.6844, pp. 346-351.

Wang, L., I. Tassiulas, K.-H. Park-Min, A. C. Reid, H. Gil-Henn, J. Schlessinger, R. Baron, J. J. Zhang, and L. B. Ivashkiv (2008). "'Tuning' of Type I Interferon-Induced Jak-STAT1 Signaling by CalciumDependent Kinases in Macrophages". In: Nat. Immunol. 9.2, pp. 186-193.

Wang, R., B. Yang, and D. Zhang (2011). "Activation of Interferon Signaling Pathways in Spinal Cord Astrocytes from an ALS Mouse Model”. In: Glia 59.6, pp. 946-958.

Wang, T., T. Town, L. Alexopoulou, J. F. Anderson, E. Fikrig, and R. A. Flavell (2004). "Toll-like Receptor 3 Mediates West Nile Virus Entry into the Brain Causing Lethal Encephalitis". In: Nat. Med. 10.12, pp. 1366-1373.

Wang, Y.-C., Y. Zhou, H. Fang, S. Lin, P.-F. Wang, R.-P. Xiong, J. Chen, X.-Y. Xiong, F.-L. Lv, Q.-L. Liang, and Q.-W. Yang (2014). "Toll-like Receptor 2/4 Heterodimer Mediates Inflammatory Injury in Intracerebral Hemorrhage". In: Ann. Neurol. 75.6, pp. 876-889.

Ward, P. A. and L. J. Newman (1969). "A Neutrophil Chemotactic Factor from Human C'5". In: J. Immunol. 102.1, pp. 93-99.

Warren, M. K. and S. N. Vogel (1985). "Bone Marrow-Derived Macrophages: Development and ReguIation of Differentiation Markers by Colony-Stimulating Factor and Interferons". In: J. Immunol. 134.2, pp. 982-989. 
Watanabe, T., N. Asano, S. Fichtner-Feigl, P. L. Gorelick, Y. Tsuji, Y. Matsumoto, T. Chiba, I. J. Fuss, A. Kitani, and W. Strober (2010). "NOD1 Contributes to Mouse Host Defense against Helicobacter Pylori via Induction of Type I IFN and Activation of the ISGF3 Signaling Pathway". In: J. Clin. Invest. 120.5, pp. 1645-1662.

Weighardt, H., S. Kaiser-Moore, S. Schlautkötter, T. Rossmann-Bloeck, U. Schleicher, C. Bogdan, and B. Holzmann (2006). "Type I IFN Modulates Host Defense and Late Hyperinflammation in Septic Peritonitis". In: J. Immunol. 177.8, pp. 5623-5630.

Wengner, A. M., S. C. Pitchford, R. C. Furze, and S. M. Rankin (2008). "The Coordinated Action of G-CSF and ELR + CXC Chemokines in Neutrophil Mobilization during Acute Inflammation". In: Blood 111.1 , pp. 42-49.

Williams, N. K., R. S. Bamert, O. Patel, C. Wang, P. M. Walden, A. F. Wilks, E. Fantino, J. Rossjohn, and I. S. Lucet (2009). "Dissecting Specificity in the Janus Kinases: The Structures of JAK-Specific Inhibitors Complexed to the JAK1 and JAK2 Protein Tyrosine Kinase Domains". In: J. Mol. Biol. 387.1, pp. 219-232.

Wright, S. D., R. A. Ramos, P. S. Tobias, R. J. Ulevitch, and J. C. Mathison (1990). "CD14, a Receptor for Complexes of Lipopolysaccharide (LPS) and LPS Binding Protein”. In: Science 249.4975, pp. 14311433.

Wrzos, C., A. Winkler, I. Metz, D. M. Kayser, D. R. Thal, C. Wegner, W. Brück, S. Nessler, J. L. Bennett, and C. Stadelmann (2014). "Early Loss of Oligodendrocytes in Human and Experimental Neuromyelitis Optica Lesions". In: Acta Neuropathol. 127.4, pp. 523-538.

Xin, L., D. A. Vargas-Inchaustegui, S. S. Raimer, B. C. Kelly, J. Hu, L. Zhu, J. Sun, and L. Soong (2010). "Type I IFN Receptor Regulates Neutrophil Functions and Innate Immunity to Leishmania Parasites". In: J. Immunol. 184.12, pp. 7047-7056.

$\mathrm{Xu}, \mathrm{J}$. and E. A. Ling (1994). "Upregulation and Induction of Surface Antigens with Special Reference to MHC Class II Expression in Microglia in Postnatal Rat Brain Following Intravenous or Intraperitoneal Injections of Lipopolysaccharide". In: J. Anat. 184 ( Pt 2), pp. 285-296.

Xu, J., L. Zhu, S. He, Y. Wu, W. Jin, T. Yu, J. Y. Qu, and Z. Wen (2015). "Temporal-Spatial Resolution Fate Mapping Reveals Distinct Origins for Embryonic and Adult Microglia in Zebrafish". In: Dev. Cell 34.6, pp. 632-641.

Yamada, T., M. A. Horisberger, N. Kawaguchi, I. Moroo, and T. Toyoda (1994). "Immunohistochemistry Using Antibodies to Alpha-Interferon and Its Induced Protein, MxA, in Alzheimer's and Parkinson's Disease Brain Tissues". In: Neurosci. Lett. 181 (1-2), pp. 61-64.

Yamamoto, M., S. Sato, H. Hemmi, K. Hoshino, T. Kaisho, H. Sanjo, O. Takeuchi, M. Sugiyama, M. Okabe, K. Takeda, and S. Akira (2003). "Role of Adaptor TRIF in the MyD88-Independent Toll-like Receptor Signaling Pathway”. In: Science 301.5633, pp. 640-643.

Yamamoto, M., S. Sato, H. Hemmi, H. Sanjo, S. Uematsu, T. Kaisho, K. Hoshino, O. Takeuchi, M. Kobayashi, T. Fujita, K. Takeda, and S. Akira (2002). "Essential Role for TIRAP in Activation of the Signalling Cascade Shared by TLR2 and TLR4". In: Nature 420.6913, pp. 324-329.

Yan, H., K. Krishnan, A. C. Greenlund, S. Gupta, J. T. Lim, R. D. Schreiber, C. W. Schindler, and J. J. Krolewski (1996). "Phosphorylated Interferon-Alpha Receptor 1 Subunit (IFNaR1) Acts as a Docking Site for the Latent Form of the $113 \mathrm{kDa}$ STAT2 Protein". In: EMBO J. 15.5, pp. 1064-1074.

Yang, C. H., W. Shi, L. Basu, A. Murti, S. N. Constantinescu, L. Blatt, E. Croze, J. E. Mullersman, and L. M. Pfeffer (1996). "Direct Association of STAT3 with the IFNAR-1 Chain of the Human Type I Interferon Receptor". In: J. Biol. Chem. 271.14, pp. 8057-8061.

Yao, J., L. Harvath, D. L. Gilbert, and C. A. Colton (1990). "Chemotaxis by a CNS Macrophage, the Microglia". In: J. Neurosci. Res. 27.1, pp. 36-42. 
Yoshimura, A., E. Lien, R. R. Ingalls, E. Tuomanen, R. Dziarski, and D. Golenbock (1999). "Cutting Edge: Recognition of Gram-Positive Bacterial Cell Wall Components by the Innate Immune System Occurs via Toll-like Receptor 2". In: J. Immunol. 163.1, pp. 1-5.

Zanoni, I., R. Ostuni, L. R. Marek, S. Barresi, R. Barbalat, G. M. Barton, F. Granucci, and J. C. Kagan (2011). "CD14 Controls the LPS-Induced Endocytosis of Toll-like Receptor 4". In: Cell 147.4, pp. 868880.

Zhan, Y., R. C. Paolicelli, F. Sforazzini, L. Weinhard, G. Bolasco, F. Pagani, A. L. Vyssotski, A. Bifone, A. Gozzi, D. Ragozzino, and C. T. Gross (2014). "Deficient Neuron-Microglia Signaling Results in Impaired Functional Brain Connectivity and Social Behavior". In: Nat. Neurosci. 17.3, pp. 400-406.

Zhou, L. J. and T. F. Tedder (1996). "CD14+ Blood Monocytes Can Differentiate into Functionally Mature CD83+ Dendritic Cells”. In: Proc. Natl. Acad. Sci. U.S.A. 93.6, pp. 2588-2592.

Ziegler-Heitbrock, L., P. Ancuta, S. Crowe, M. Dalod, V. Grau, D. N. Hart, P. J. M. Leenen, Y.-J. Liu, G. MacPherson, G. J. Randolph, J. Scherberich, J. Schmitz, K. Shortman, S. Sozzani, H. Strobl, M. Zembala, J. M. Austyn, and M. B. Lutz (2010). "Nomenclature of Monocytes and Dendritic Cells in Blood". In: Blood 116.16, e74-80.

Ziv, Y., N. Ron, O. Butovsky, G. Landa, E. Sudai, N. Greenberg, H. Cohen, J. Kipnis, and M. Schwartz (2006). "Immune Cells Contribute to the Maintenance of Neurogenesis and Spatial Learning Abilities in Adulthood". In: Nat. Neurosci. 9.2, pp. 268-275. 